\title{
Final technical report for the DOE ARM project "Improving the understanding of surface-atmosphere radiative interactions by mapping surface reflectance over the ARM CART site”
}

\author{
Award number: DE-FG02-02ER63351 \\ Project period: March 2002 - June 2005
}

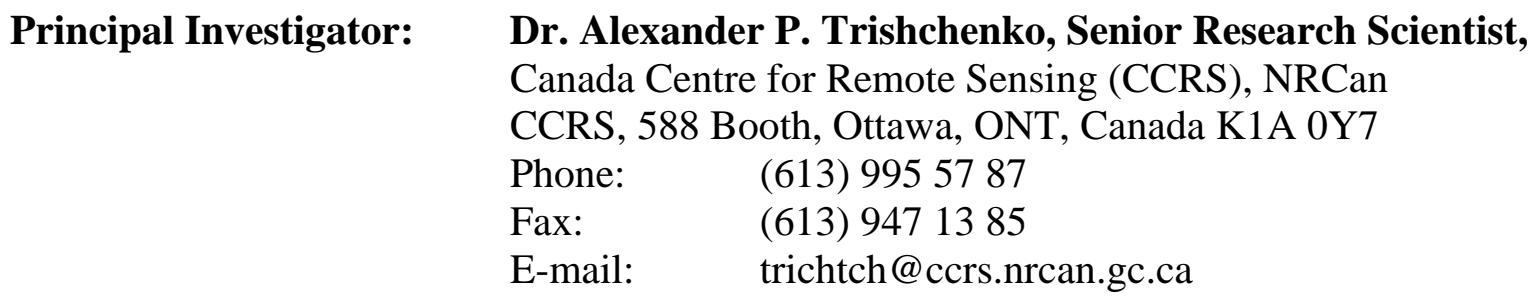

Co-investigators:

Dr. Yi Luo, Dr. K. Khlopenkov, Dr. W. B. Park, Canada Centre for Remote Sensing, Ottawa, Canada

\section{Collaborators:}

Prof. Z. Li, Ms. M. C. Cribb,

Earth System Science Interdisciplinary Center, University of Maryland, MD, USA

Ottawa, November, 2008 


\begin{abstract}
:
Surface spectral reflectance (albedo) is a fundamental variable affecting the transfer of solar radiation and the Earth's climate. It determines the proportion of solar energy absorbed by the surface and reflected back to the atmosphere. The International Panel on Climate Change (IPCC) identified surface albedo among key factors influencing climate radiative forcing. Accurate knowledge of surface reflective properties is important for advancing weather forecasting and climate change impact studies. It is also important for determining radiative impact and acceptable levels of greenhouse gases in the atmosphere, which makes this work strongly linked to major scientific objectives of the Climate Change Research Division (CCRD) and Atmospheric Radiation Measurement (ARM) Program.
\end{abstract}

Most significant accomplishments of eth project are listed below.

I) Surface albedo/BRDF datasets from 1995 to the end of 2004 have been produced. They were made available to the ARM community and other interested users through the CCRS public ftp site ftp://ftp.ccrs.nrcan.gc.ca/ad/CCRS_ARM/ and ARM IOP data archive under "PI data Trishchenko".

II) Surface albedo properties over the ARM SGP area have been described for 10-year period. Comparison with ECMWF data product showed some deficiencies in the ECMWF surface scheme, such as missing some seasonal variability and no dependence on sky-conditions which biases surface energy budget and has some influence of the diurnal cycle of upward radiation and atmospheric absorption.

III) Four surface albedo Intensive Observation Period (IOP) Field Campaigns have been conducted for every season (August, 2002, May 2003, February 2004 and October 2004). Data have been prepared, documented and transferred to ARM IOP archive.

Nine peer-reviewed journal papers and 26 conference papers have been published. 
Surface spectral reflectance (albedo) is a fundamental variable affecting the transfer of solar radiation and the Earth's climate. It determines the proportion of solar energy absorbed by the surface and reflected back to the atmosphere. The International Panel on Climate Change (IPCC) identified surface albedo among key factors influencing climate radiative forcing. Accurate knowledge of surface reflective properties is important for advancing weather forecasting and climate change impact studies. It is also important for determining radiative impact and acceptable levels of greenhouse gases in the atmosphere, which makes this work strongly linked to major scientific objectives of the Climate Change Research Division (CCRD) and Atmospheric Radiation Measurement (ARM) Program.

Most significant accomplishments of the project are listed below.

I) Surface albedo/BRDF datasets from 1995 to the end of 2004 have been produced. They were made available to the ARM community and other interested users through the CCRS public ftp site ftp://ftp.ccrs.nrcan.gc.ca/ad/CCRS_ARM/ and ARM IOP data archive under "PI data Trishchenko". Surface albedo and BRDF products were generated every 10-days from MODIS 2000-2004 (500m spatial resolution), VGT/SPOT 1998-2004 (1 km resolution), AVHRR 1995-2000 (1 km resolution). Data product intercomparison and validation studies have been conducted.

Surface bi-directional reflectance distribution function (BRDF) and albedo maps are derived from Moderate-resolution Imaging Spectroradiometer (MODIS) multi-day surface reflectance composites with a 500-meter spatial resolution (MODIS product MOD09A1/MYD09A1). The proposed method dubbed as landcover-based fitting (LBF) employs the processing of clear-sky reflectance data for similar landcover types and takes into account the magnitude of normalized difference vegetation index (NDVI) (Luo et al., 2005). The BRDF is derived through the fitting of pixel data sorted into small bins according to the values of angular variables and NDVI. Robust statistical processing is applied to reduce the influence of noise and outliers. This method increases the success rate of the fitting process and enables more accurate monitoring of surface temporal changes during periods of rapid spring vegetation green-up and autumn leaf-fall, as well as changes due to agricultural practices and snow-cover variations. The approach is specifically applied over the Atmospheric Radiation Measurement (ARM) Program Southern Great Plains (SGP) area. Results are compared to alternative BRDF/albedo products, such as the MOD43 albedo and MISR surface products that are derived through a pixel-based fitting process. A good agreement was generally found between different data sets. For example, the average biases in the visible and near-infrared bands are usually less than 0.01 and 0.02 , and correlation coefficients are typically larger than 0.80 . An analysis of these differences identifies some unique advantages of proposed method, such as the ability to capture rapidly changing surface properties and an increased performance in the case of reduced number of clear sky-observations due to frequent cloudy conditions. Results suggest that the 
developed landcover-based methodology is valuable for the purpose of surface BRDF and albedo mapping using MODIS observations.

A method was developed to obtain time series of the surface albedo characteristics consistent with MODIS using historical satellite observations with limited spectral coverage available from AVHRR/NOAA and VEGETATION/SPOT (Trishchenko et al., 2007). Non-linear regression model was developed that relates retrievals from four spectral channels of VEGETATION/SPOT or three spectral channels of AVHRR/NOAA with retrieval from each of the seven MODIS channels designed for land applications. The model also takes into account the surface land cover type, normalized difference vegetation index and seasonal cycle. It was applied to generate surface albedo and bi-directional parameters of the seven MODIS like spectral channels at a 10-day interval for the 1995 to 2004 period over the US Southern Great Plains. The relative retrieval accuracy for MODIS channels replicated from AVHRR or VGT data was typically better than $5 \%$. Correlation coefficients between replicated and original data varied from 0.92 to 0.98 for all channels except MODIS channel 5 , where it was lower (0.77 to 0.84 ). The developed method provides valuable information for parameterization of spectral albedo in global climate models and can be extended to generate global multi-spectral data compatible with MODIS from historical AVHRR and VEGETATION/SPOT observations for the pre-MODIS era.

\section{II) Surface albedo properties over the ARM SGP area have been described for 10-year} period. Comparison with ECMWF data product showed some deficiencies in the ECMWF surface scheme, such as missing some seasonal variability and no dependence on sky-conditions which biases surface energy budget and has some influence of the diurnal cycle of upward radiation and atmospheric absorption. Natural variability and sampling errors in solar radiation measurements over the ARM/SGP region have been analyzed (Trishchenko et al., 2008, Li et al., 2005).

For the general purpose of evaluating a model's performance, results from a hierarchy of models have been compared against radiation measurements made at the Atmospheric Radiation Measurement Southern Great Plains region. Important questions were raised as to 1) how well the measurements represent model grid mean values, 2) on what scale does modeling need to occur in order to capture a physical process, and 3) how much of model-observation differences are attributed to inherent sampling errors. We attempt to address some of these questions using surface solar radiation data obtained from ground and satellites. Observation uncertainties caused by cloud variability at different scales in different seasons were characterized, which can guide modelobservation comparisons. In comparing single-point measurements against area mean satellite retrievals, much of the differences are caused by ground sampling errors. This implies that the inherent sampling errors must be taken into consideration when comparing model-simulated grid mean fluxes against ground-based point-specific observations. The sampling errors in different months, model domain sizes and integration intervals are quantified. For example, for March 2000, 
one may not expect model simulated instantaneous fluxes and the CF measurements to agree within $16 \mathrm{Wm}-2$ for a model grid of $10 \times 10 \mathrm{~km} 2$; the best accuracy increases to $46 \mathrm{~W} \mathrm{~m} \mathrm{-2} \mathrm{and} 64 \mathrm{~W} \mathrm{~m}-2$ if the model grid increases to 200x200 km 2 and 400x400 km 2 , respectively. The sampling uncertainties decrease rapidly as the averaging interval increases. For daily mean fluxes, for example, the sampling error is about $16 \mathrm{Wm}-2$ in September 2000 over a typical general circulation model grid of 200x200 km 2 . In general, for averaging intervals less than a day, the sampling error decreases sharply. Averaging over periods longer than 5 days reduces the error to a magnitude of less than 15Wm-2 over all domain sizes. While the statistics presented here vary with time and region, the method proposed should be valid in general.

III) Four surface albedo Intensive Observation Period (IOP) Field Campaigns have been conducted for every season (August, 2002, May 2003, February 2004 and October 2004). Data have been prepared, documented and transferred to ARM IOP archive. The systematic measurements of surface albedo properties were conducted over an extended area around the Atmospheric Radiation Measurement (ARM) Program Southern Great Plains (SGP) site as a part of the ARM Aerosol Intensive Observational Period (IOP) organized in May 2003. Albedo is required as surface boundary condition for assessment of aerosol radiative forcing and aerosol satellite retrievals, modeling of radiative transfer, atmospheric dynamics and clouds. Ground and satellite observations of the surface spectral and angular properties were collected. Detailed intercomparison was made between multiplatform measurements available from the ground, satellite and aircraft observations. Results in general showed good consistency between various sources. High-temporal resolution observations (10 day intervals or shorter ) are required to represent rapid changes of surface albedo properties over the ARM SGP CART site area detected during the springtime.

\section{Publications and presentations. Peer-reviewed journal papers:}

1. Trishchenko, A.P., Y.Luo, K.Khlopenkov, S.Wang (2008): A method to derive the multi-spectral surface albedo consistent with MODIS from historical AVHRR and VGT satellite data. Journal of Applied Meteorology and Climatology. 47(4), 1199-1221.

2. Luo, Y., A.P. Trishchenko, R.Latifovic, Z.Li, 2005: Surface bidirectional reflectance and albedo properties derived using a land cover-based approach with Moderate Resolution Imaging Spectroradiometer observations. J. Geophys. Res., Vol. 110, No. D1, D01106. 10.1029/2004JD004741. 12 January 2005

3. Trishchenko, A.P. 2005: Solar constants and effective brightness temperatures for SW IR channels of AVHRR/NOAA and GOES imagers. Submitted to Journal of Atmospheric and Oceanic Technology. January 2005.

4. Li, Z., M.Cribb, F-L.Chang, A.P.Trishchenko, Y.Luo, 2005: Natural variability and sampling errors in radiation measurements in the context of model validation and parameterization. Journal of Geophysical Research, in press. 
5. Halthore, R., D.Crisp, ... A.P.Trishchenko \&20 other authors, 2005: Intercomparison of Shortwave Radiative Transfer Codes and Measurements, Journal of Geophysical Research. In press.

6. Barker H. W., A. Marshak, W. Szymer, A. P. Trishchenko, J.-P. Blanchet, Z.Li, 2002: Remote sensing of cloud properties from aircraft-based measurements. Part I. Optical depth. Journal of Atmospheric Sciences, 59, 2093-2111.

7. Li, Z., M. Cribb, A. Trishchenko, 2002: Impact of surface inhomogeneity on the closure of solar radiative transfer and a method of estimating surface areal-mean albedo from downwelling flux measurements. Journal of Geophysical Research. 107 (D16), 4317, doi:10.1029/2001JD000976, 2002.

8. Trishchenko, A. P., J. Cihlar, Z. Li, 2002: Effects of spectral response function on surface reflectance and NDVI measured with moderate resolution satellite sensors. Remote Sensing of Environment. 81, 1-18.

9. Trishchenko A.P., G. Fedosejevs, Z.Li, J.Cihlar, 2002: Trends and uncertainties in thermal calibration of AVHRR radiometers onboard NOAA-9 to 16. Journal of Geophysical Research. (in press). 107 (D24), 4778, doi:10.1029/2002JD002353, 2002.

\section{Conference papers and other publications}

1) Trishchenko, A.P., 2005: Solar spectrum: Uncertainties between current models and implications for atmospheric radiation modeling and remote sensing. ARM $15^{\text {th }}$ Science Team Meeting. Daytona Beach. FL. March 2005.

2) Trishchenko, A.P., Y.Luo, Z.Li, 2005: Spectral, temporal and spatial properties of surface BRDF/albedo over the ARM SGP area from multi-year satellite observations. ARM $15^{\text {th }}$ Science Team Meeting. Daytona Beach. FL. March 2005.

3) Li, Z., H.Barker, A.P.Trishchenko., 2005: A Versatile Two-Stream Model for Retrieving Surface Spectral Albedos. ARM 15 ${ }^{\text {th }}$ Science Team Meeting. Daytona Beach. FL. March 2005.

4) Li, Z., M.Cribb, F-L. Chang, A.P.Trishchenko, Y.Luo, 2005: Improving satellite estimates of cloud cover using whole-sky imager cloud cover data at the three ARM sites. ARM $15^{\text {th }}$ Science Team Meeting. Daytona Beach. FL. March 2005.

5) Li, Z., M.C.Cribb, A.P. Trishchenko, Y.Luo, 2004: Analysis of Cloud Variability and Sampling Errors in Surface and Satellite Measurements. 14th ARM Science Team Meeting. Albuquerque. NM. USA. March. 2004. http://www.arm.gov/publications/proceedings/conf14/extended_abs/li2-z.pdf

6) Li, Z., M.C.Cribb, F-L.Chang, A.P. Trishchenko, 2004: Validation of MODIS-Retrieved Cloud Fractions Using Whole-Sky Imager Measurements at the Three ARM Sites. 14th ARM Science Team Meeting. Albuquerque. NM. USA. March. 2004. http://www.arm.gov/publications/proceedings/conf14/extended_abs/li1-z.pdf

7) Luo, Y., A.P.Trishchenko, R.Latifovic, K.Khlopenkov, Z.Li, 2004: Spatially and Temporally Complete Surface Albedo Product Over the ARM SGP Area for 2000-2003 Period. 14th ARM Science Team Meeting. Albuquerque. NM. USA. March. 2004. http://www.arm.gov/publications/proceedings/conf14/extended_abs/luo-y.pdf

8) Trishchenko, A. P., K.Khlopenkov, Y.Luo, 2004: Observations during 3rd Surface Albedo IOP. Final IOP report submitted to Atmospheric Radiation Measurement (ARM) Program archive. 31pp. 
9) Trishchenko, A.P., Y.Luo, R. Latifovic, Z.Li, 2004: Land Cover Type Distribution Over the ARM SGP Area for Atmospheric Radiation and Environmental Research. 14th ARM Science Team Meeting. $\quad$ Albuquerque. $\quad$ NM. $\quad$ USA. $\quad$ March. 2004. http://www.arm.gov/publications/proceedings/conf14/extended_abs/trishchenko3-ap.pdf

10) Trishchenko, A.P., K.Khlopenkov, Y.Luo, 2004. BRDF and Albedo Properties of Pure and Mixed Surface Types From MODIS and MISR Using Landsat High-Resolution Land Cover and Angular Unmixing Technique. 14th ARM Science Team Meeting. Albuquerque. NM. USA. March. 2004. http://www.arm.gov/publications/proceedings/conf14/extended_abs/trishchenkoap.pdf

11) Trishchenko, A.P., Y. Luo, K. Khlopenkov,Z. Li, 2004: Dynamics of the Surface Albedo Over the ARM SGP Area During Spring 2003 Aerosol IOP. Proc. 14th ARM Science Team Meeting. Albuquerque. $\quad$ NM. USA. 2004. http://www.arm.gov/publications/proceedings/conf14/extended_abs/trishchenko2-ap.pdf

12) Trishchenko, A.P., K.Khlopenkov, Y.Luo (2004), Retrieval of BRDF for pure landcover types from MODIS and MISR using angular unmixing approach. In Weather and Environmental Satellites, edited by T.H.Vonder Haar, H-L . A. Huang. Proc. SPIE 5549, (SPI Bellingham, WA, 2004), pp.167-175.

13) Trishchenko, A. P., K.Khlopenkov, Y.Luo, 2004: Observations during 3rd Surface Albedo IOP. Final IOP report submitted to Atmospheric Radiation Measurement (ARM) Program archive. 31pp.

14) Trishchenko, A. P., K.Khlopenkov, Y.Luo, 2004: Observations during 4rd Surface Albedo IOP. Final IOP report submitted to Atmospheric Radiation Measurement (ARM) Program archive. $15 p p$

15) Trishchenko, AP, Y Luo, K Khlopenkov, and Z Li. 2004. Surface albedo over the ARM SGP area during spring 2003 aerosol IOP from ground and satellite observations and its impact on the solar radiative transfer. Presented at International Radiation Symposium 2004 IRS. Busan, Korea.

16) Luo, Y, AP Trishchenko, R Latifovic, K Khlopenkov, and Z Li. 2004. "Satellite retrieval and validation of surface albedo over the ARM SGP area at medium spatial resolution." Presented at International Radiation Symposium 2004 IRS. Busan, Korea.

17) Trishchenko, A.P. 2004. Uncertainties of satellite surface albedo and BRDF retrievals due to limited angular sampling. Presented at International Radiation Symposium 2004 IRS. Busan, Korea.

18) Trishchenko, A.P., Luo, Y., Cribb, M.C., Li, Z., and Hamm, K., 2003: Surface Spectral Albedo IOP at the ARM SGP site in August 2002: Results, analysis and future plans: Proc. $13^{\text {th }} A R M$ Science Team Meeting (http://www.arm.gov/docs/documents/technical/conf_0304/trishchenko-ap.pdf).

19) Li, Z., M.C. Cribb, A. Trishchenko' 2003: Characterization of Broadband Surface Albedo Properties over the ARM the North Slope of Alaska (NSA) and the Southern Great Plains (SGP) Cloud and Radiation Testbed. Proc. $13^{\text {th }}$ ARM Science Team Meeting http://www.arm.gov/docs/documents/technical/conf 0304/li-z.pd

20) Li,Z., Cribb,M.C., and Trishchenko,A.P., 2003: Toward the Development of Multi-Year Total and Spectral Solar Radiation Budgets at the Three ARM Locales. Proc. $13^{\text {th }}$ ARM Science Team Meeting (http://www.arm.gov/docs/documents/technical/conf_0304/li2-z.pdf)

21) Luo, Y., Trishchenko, A.P., Latifovic, R., and Li, Z., 2003: Seasonal dynamics of surface bidirectional reflectance properties over the ARM SGP area from satellite multi-platform observations. $\quad$ Proc. $13^{\text {th }} \quad$ ARM Science Team Meeting (http://www.arm.gov/docs/documents/technical/conf 0304/luo-y.pdf) 
22) Luo Y, A.P. Trishchenko, R. Latifovic, Z. Li, 2003: Albedo/BRDF Retrievals from MODIS Using 8-day Clear-sky Composites. Eos Trans. AGU, 84(46), Fall Meet. Suppl., Abstract B21E0763.

23) Trishchenko, A. P., 2002: Asymmetry in the diurnal cycle of atmospheric downwelling radiation at the ARM SGP CF site over 1995-2001 Period. Proc. $12^{\text {th }}$ ARM Science Team Meeting, St. Petersburg, FLA, http://www.arm.gov/docs/documents/technical/conf 0204/trishchenko(2)-ap.pdf.

24) Trishchenko, A. P., B. Hwang, Z. Li, 2002: Atmospheric correction of satellite signal in solar domain: Impact of improved molecular spectroscopy. Proc. $12^{\text {th }}$ ARM Science Team Meeting, St. Petersburg, FLA http://www.arm.gov/docs/documents/technical/conf 0204/trishchenko-ap.pdf.

25) Trishchenko, A. P., Y. Luo, R. Latifovic, W. Park, J. Cihlar, B. Hwang, Z. Li, M. Cribb, 2002: Towards development of a synthesized database of spatial and temporal surface Spectral reflectivity over the ARM SGP CART area. Proc. $12^{\text {th }}$ ARM Science Team Meeting, St. Petersburg, FLA, http://www.arm.gov/docs/documents/technical/conf_0204/trishchenko(3)ap.pdf.

26) Trishchenko, A. P., Y. Luo, R. Latifovic, W. Park, J. Cihlar, Z. Li, 2002: Mapping surface reflective properties over the ARM SGP CART area by combining multi-platform observational datasets. Proc. 11-th AMS Conference on Atmospheric Radiation. Ogden. Utah. p.82-85. 
Appendices: Copy of publications. 


\title{
Surface bidirectional reflectance and albedo properties derived using a landcover based approach with MODIS observations
}

\author{
Yi Luo \\ Noetix Research Inc., 265 Carling Ave., Ottawa, Ontario, Canada, K1S2E1 \\ Alexander P. Trishchenko*, and Rasim Latifovic \\ Canada Centre for Remote Sensing, Natural Resources Canada, 588 Booth Street, Ottawa, \\ Ontario, Canada, K1A0Y7 \\ Zhanqing Li \\ Earth System Science Interdisciplinary Center, University of Maryland, College Park, MD, \\ 20742, USA \\ * Corresponding author: Tel 1-613-995 57 87; fax: 1-613-947 1383 \\ E-mail: trichtch@ccrs.nrcan.gc.ca
}

\author{
Submitted to \\ Journal of Geophysical Research
}

March 8, 2004

Revised August 14, 2004 


\begin{abstract}
Surface bi-directional reflectance distribution function (BRDF) and albedo maps are derived from Moderate-resolution Imaging Spectroradiometer (MODIS) multi-day surface reflectance composites with a 500-meter spatial resolution (MODIS product MOD09A1/MYD09A1). The proposed method dubbed as landcover-based fitting (LBF) employs the processing of clear-sky reflectance data for similar landcover types and takes into account the magnitude of normalized difference vegetation index (NDVI). The BRDF is derived through the fitting of pixel data sorted into small bins according to the values of angular variables and NDVI. Robust statistical processing is applied to reduce the influence of noise and outliers. This method increases the success rate of the fitting process and enables more accurate monitoring of surface temporal changes during periods of rapid spring vegetation green-up and autumn leaf-fall, as well as changes due to agricultural practices and snow-cover variations. The approach is specifically applied over the Atmospheric Radiation Measurement (ARM) Program Southern Great Plains (SGP) area. Results are compared to alternative BRDF/albedo products, such as the MOD43 albedo and MISR surface products that are derived through a pixel-based fitting process. A good agreement was generally found between different data sets. For example, the average biases in the visible and near-infrared bands are usually less than 0.01 and 0.02 , and correlation coefficients are typically larger than 0.80 . An analysis of these differences identifies some unique advantages of proposed method, such as the ability to capture rapidly changing surface properties and an increased performance in the case of reduced number of clear sky-observations due to frequent cloudy conditions. Results suggest that the developed landcover-based methodology is valuable for the purpose of surface BRDF and albedo mapping using MODIS observations.
\end{abstract}


Index Terms: 3322 Land/atmosphere interactions; 3359 Radiative processes; 3360 Remote sensing

Keywords: Surface albedo, bi-directional reflectance, BRDF, MODIS, ARM SGP, landcoverbased fitting 


\section{Introduction}

Surface albedo is defined as the ratio of the total (hemispheric) reflected solar radiation flux to the incident flux upon the surface. It is one of the most important parameters in atmospheric radiation studies. Albedo is important because even small changes in its magnitude can lead to variations in radiative forcing, to which climate is extremely sensitive [Ingram et al., 1989; Houghton et al., 2001; Dirmeyer and Shukla, 1994]. The spatial complexity and temporal variations of surface properties mean that practical albedo estimates over large area are usually derived from satellite observations [Strugnell et al., 2001; Cihlar et al., 2002; Gutman et al., 1989]. Most land surfaces reflect incident radiation anisotropically. Anisotropic properties are described by the surface bi-directional reflectance distribution function (BRDF) [Nicodemus et al., 1977]. An accurate determination of surface BRDF properties is essential because they are directly related to the accuracy of derived surface albedo and radiation fluxes, which are obtained as hemispheric integrals of BRDF and radiance fields. The retrieval of surface BRDF is not a simple task since most space-borne sensors only view the ground target at limited observational geometries that are defined by the solar zenith angle (SZA), viewing zenith angle (VZA), and sun-satellite relative azimuth angle (RAA) [Csiszar et al., 2001].

There are many parametric BRDF models that are based on the physical characteristics and experimental results observed for natural surfaces [Lucht and Roujean, 2000]. Semiempirical linear kernel-driven models are among the most widely used of these. Kernel-driven models are attractive because of their fast operational implementation and good accuracy [Wu et al., 1995; Wanner et al., 1997; Privette et al., 1997; Lucht et al., 2000]. The derivation of BRDF parameters - i.e. the process of model inversion - is achieved through a fitting procedure that tunes the model to observed data points by minimizing mean-square residuals. For a successful inversion, the observations must cover a range of sun-target-viewer geometries (and ideally, the 
entire range). In practice, however, this requirement is rarely achieved. For a fixed location on the Earth's surface, some satellite systems, such as the Multiangle Imaging Spectro-Radiometer (MISR), can obtain multiple angular views virtually instantaneously, while others, such as the Moderate-resolution Imaging Spectroradiometer (MODIS), build up sequential angular views over a period of time. The latter approach - hereafter referred to as a pixel-based fitting (PBF) method - is currently implemented in the MODIS operational albedo retrieval algorithm (MOD43). It provides surface albedo for each $1 \mathrm{~km}$ pixel at 16-day intervals [Schaaf et al., 2002]. This 16-day sampling period is deemed to be long enough to acquire a number of clear-sky observations over a range of sun-target-viewer geometries. However, a shorter sampling period may be required by applications where surface properties vary rapidly over short timescales (e.g. during periods of snow fall, snow melt, and soil moisture and vegetation change). In such cases, the shortening of the time interval in the PBF approach reduces the number of clear-sky data points used in the fitting procedure. This makes the retrieval of albedo less reliable or may result in a failure of the inversion procedure. Even for a 16-day sampling interval and for a maximal potential number of clear-sky data points included in the inversion procedure, the retrievals may still fail or produce biased results due to intrinsic noise in the data [Jin et al., 2003a]. Moody et al. [2004] demonstrated that there is a pressing need to improve the spatial completeness of the MODIS operational product due to significant gaps in the data. About $50 \%$ of useful data are missing on a global scale due to insufficient sampling of clear-sky pixels for BRDF/Albedo retrievals.

A landcover-based fitting (LBF) approach is presented in this paper. This method reduces the difficulties associated with a small number of data points in the fitting procedure, and produces BRDF/albedo products for shorter time intervals. Our approach employs the similarity 
in the BRDF properties of varioous pixels, if these pixels belong to the same land cover type, are in a similar biophysical condition, and occur within the same climatic region [Trishchenko et al., 2004, Latifovic et al., 2003]. For any given region of interest, clear-sky pixels and land cover information are used to derive BRDF model parameters for each landcover type in the region. The LBF approach overcomes the limitations of PBF approaches that are associated with a small or insufficient amount of data used in the fitting process. It does this by (a) providing improved sampling in terms of the number of observations and range of geometries used, (b) requiring less computational resources than other approaches. The LBF method can be implemented for shorter time intervals, that enables to capture a rapid change in BRDF characteristics. The potential limitation of this approach is a reduced sensitivity to subtle spatial differences in BRDF shape within the same landcover class.

This paper describes and applies the LBF methodology to the MODIS surface reflectance product (MOD09A1 for Terra and MYD09A1 for Aqua) [Vermote and Vermeulen, 1999]. The MOD09A1/MYD09A1 product contains the MODIS surface reflectance composite data generated at a 500-meter spatial resolution and at 8-day intervals. This product also includes angular variables, such as SZA, VZA, and RAA, as well as the date of observation, quality flags and a variety of auxiliary information (http://edcdaac.usgs.gov/modis/mod09a1v4.html). The LBF methodology is tested over the Southern Great Plains (SGP) region. This region is in the focus of the Atmospheric Radiation Measurement (ARM) program (http://www.arm.gov). The ARM SGP site serves as an important test bed for the physics of climate and climate change research, atmospheric radiation budget and weather/climate models [Ackerman and Stokes, 2003]. Spatially and temporally complete high quality surface BRDF/albedo maps of this area will greatly assist the ARM program to achieve its major objective "to improve understanding of the 
processes and properties that affect atmospheric radiation" [Stokes and Schwartz, 1994]. The understanding and modeling of atmospheric radiation and cloud processes may be improved in climate and weather prediction studies by taking into account realistic albedo variations with solar zenith angle, landcover type, and seasonal vegetation phenology [Lucht and Roujean, 2000].

The structure of this paper is as follows: the general methodology and its realization are described in Section 2. This includes the basic concept of the BRDF LBF methodology, data description, BRDF model specification, and implementation procedure. Section 3 analyses some typical BRDF results for the ARM SGP area. Comparisons of LBF BRDF/albedo data with other products, such as MODIS MOD43B1 and MISR albedo, are presented in Section 4. Section 5 contains conclusions and final discussion.

\section{BRDF Landcover Based Fitting (LBF) Methodology}

\subsection{Basic Concept}

Surface BRDF is physically determined by the geometric structure of the land surface as well as the optical properties of canopy elements, understory, and soil conditions. Several parametric BRDF models have been developed based on various assumptions about these properties [e.g. Roujean et al., 1992; Li and Strahler, 1992; Rahman et al., 1993]. Surface structure influences the BRDF through the reflectors' geometric features, their shadowcasting and their spatial distribution. Optical characteristics account for the absorbing and scattering effects that the surface elements exert on the radiation field. The BRDF model parameters are closely related to landcover type [e.g. Wu et al., 1995; Lucht et al., 2000]. As shown by Trishchenko et al. [2004] and Latifovic et al. [2003] it is reasonable to assume that the BRDF 
shape is generally similar for the same landcover type under similar biophysical conditions characterized by the Normalized Difference Vegetation Index (NDVI).

The LBF approach uses a multi-day clear-sky composite of surface reflectance and groups pixels by landcover type and NDVI values. Landcover and NDVI data are both required for the successful implementation of the LBF approach. Grouped data are then used to optimally fit the BRDF model parameters for each landcover type. After these generic BRDFs are obtained, pixel-level BRDFs are determined by adjusting the general landcover BRDF to the observed reflectances of a given pixel. A similar strategy was successfully employed for BRDF/albedo retrievals from Advance Very High Resolution Radiometer (AVHRR) and SPOTVEGETATION data [Cihlar et al., 2002; Cihlar et al., 2004; Latifovic et al., 2003; Latifovic et al., 2004; Li et al. 1996].

\subsection{Landcover Map over the ARM SGP Area}

The National Land Cover Dataset (NLCD) is used in this study. The NLCD was compiled from Landsat Thematic Mapper (TM) satellite imagery at 30-meter spatial resolution and supplemented by various ancillary data. It was produced as part of a cooperative project between the U.S. Geological Survey (USGS) and the U.S. Environmental Protection Agency (USEPA) (http://landcover.usgs.gov/natllandcover.asp). The original NLCD landcover dataset was resampled to create a landcover map that matched the spatial resolution of the MODIS data used in this study (500 meters). Each landcover class in the resampled dataset was then converted to its International Geosphere-Biosphere Programme (IGBP) equivalent following the approach described by Latifovic et al. [2004]. The IGBP classification was selected because of its frequent use in climate modeling and ecological studies. The preference was given to the 
NLCD landcover map rather than the MODIS land cover product (MOD12) [Strahlar et al., 1999] because the latter is only available at a spatial resolution of $1 \mathrm{~km}$, and because the use of the former avoids potential biases and correlation impacts between products from the same sensor.

The study region covers an $8^{\circ}$ latitude $\times 10^{\circ}$ longitude area centered at the ARM SGP Central Facility (CF) located in Northern Oklahoma, US. Figure 1 shows the spatial distribution of the 10 IGBP landcover classes that occur in the region. The area is dominated by grasslands (53\%), croplands (19\%) and deciduous forest (13\%). Less frequent landcover types include evergreen forest (3.9\%) and open shrublands (3.5\%). While cropland is the main landcover type around the $\mathrm{CF}$, it is mixed with grasslands in the western and northern parts of the study area. In addition, shrublands are present in the southwest part of the study area, while the southeasternmost portion of the study area is dominated by forest. Usually, the dominant landcover types are geographically concentrated over relatively uniform area.

\subsection{BRDF Shape and NDVI}

The BRDF of the land surface is dependent on both landcover (vegetation) type and vegetation condition [e.g. Wu et al., 1995; Li et al. 1996]. The vegetation condition can be described by the magnitude of the NDVI [Gutman, 1987]. Data collected from fine-resolution Landsat imagery and the MISR 1-km surface product illustrate the impact of NDVI on BRDF. Figure 2a shows a Landsat-7 ETM RGB color composite acquired over the ARM SGP CF area on March 9 (day 68) 2002. The image contains a mixture of cropland and grassland (pasture) according to field surveys for that area [Li et al., 2002]. Analysis uses MISR observations over a $2 \times 2 \mathrm{~km}^{2}$ area of cropland close to the CF to illustrate the variability in surface BRDF properties 
with NDVI (Figure 2a). The MISR sensor is well suited for such an analysis because it views the target at nine angles almost instantaneously with a footprint size of 1.1-km [Diner et al., 1999]. The MISR spatial resolution is, however, somewhat too coarse to resolve the spatial variation of the BRDF inherent to various crop types. This may reduce the actual contrast between various BRDFs. The selected sampling area corresponds to a group of $2 \times 2$ pixels in the MISR data. The observations for each pixel are also shown in Figure 2 as a function of the RAA vs. VZA (Figure 2b), surface reflectance vs. VZA for the red band (661-683 nm) (Figure 2c) and surface reflectance vs. VZA for the NIR band (846-886 nm) (Figure 2d). The averaged NDVI values (within $\pm 50^{\circ}$ VZA) are also given in Figure $2 \mathrm{~b}$. Figures $2 \mathrm{~b}-2 \mathrm{~d}$ show the MISR reflectances for the same day as that of the Landsat image. Figures $2 \mathrm{e}-2 \mathrm{~g}$ show the MISR reflectances three weeks later (April 1 (day 91), 2002). The SZAs for day 68 and 91 are approximately $44^{\circ}$ and $34^{\circ}$, respectively. Figure 2 shows that the magnitude of NDVI defines the BRDF shape in a unique way. For day 68, pixels 1 and 3 have similar NDVI and reflectances values. Pixels 2 and 4, however, have an NDVI difference of nearly 0.2 , which corresponds to the large difference in vegetation density seen in Figure 2a. The reflectances and BRDF profiles of these pixels are thus substantiality different, especially in the red band. Specifically, the red band reflectance for pixel 4 is strongest for the backscattered viewing geometry when the VZA is close to the SZA. It should also be noted that the temporal trend in the surface properties, even after only three weeks, is considerable. This reflects a rapid change in vegetation properties during the peak of the growing season as confirmed by the magnitudes of NDVI between these dates. This emphasizes the advantage of having shorter sampling intervals than the 16-day period currently adopted in the operational MODIS BRDF/Albedo processing. A shorter sampling interval is especially 
important during periods when surface properties change rapidly, or when detailed knowledge of phenological change is required.

\subsection{RossThick-LiSparse Reciprocal BRDF Model}

The RossThick-LiSparse model (hereafter referred to as Ross-Li) is employed in this study. The Ross-Li model has been identified as the most appropriate model for the operational processing of MODIS data [Wanner et al., 1997; Privette et al., 1997; Li et al., 1997; Lucht and Lewsi, 2000]. It has also been demonstrated that the Ross-Li and the nonlinear Rahman's models [Rahman et al., 1993; Martonchik, 1997] perform similarly. However, the linear Ross-Li model is more computationally efficient.

The linear BRDF model is expressed as a sum of several theoretically constructed kernel functions $f_{i}\left(\theta_{s}, \theta_{v}, \phi\right)$, where $\theta_{s}, \theta_{v}$ and $\phi$ are the SZA, VZA and RAA, respectively. The reciprocal model of Ross-Li [Wanner et al., 1995] is a model that uses 3 kernels

$$
\rho_{\lambda}\left(\theta_{s}, \theta_{v}, \phi\right)=a_{0}+a_{1} f_{1}\left(\theta_{s}, \theta_{v}, \phi\right)+a_{2} f_{2}\left(\theta_{s}, \theta_{v}, \phi\right)
$$

where $f_{1}$ is the RossThick kernel and represents volumetric scattering from a dense leaf canopy based on a single-scattering approximation of radiative transfer theory [Ross, 1981; Roujean et $a l ., 1992] . f_{2}$ is the LiSparse kernel which is derived from the geometric-optical mutual shadowing model, and assumes a sparse ensemble of surface objects [Li and Strahler, 1992]. Parameters $a_{0}, a_{1}$ and $a_{2}$ are coefficients of the kernels and are related to the isotropic, volumetric and geometric components, respectively. Kernels are given by the following expressions

$$
f_{1}=[(\pi / 2-\xi) \cos \xi+\sin \xi] /\left[\cos \theta_{s}+\cos \theta_{v}\right]-\pi / 4
$$


where $\cos \xi=\cos \theta_{s} \cos \theta_{v}+\sin \theta_{s} \sin \theta_{v} \cos \phi$

$$
f_{2}=O\left(\theta_{s}, \theta_{v}, \phi\right)-\sec \theta_{s}^{\prime}-\sec \theta_{v}^{\prime}+\frac{1}{2}\left(1+\cos \xi^{\prime}\right) \sec \theta_{s}^{\prime} \sec \theta_{v}^{\prime}
$$

$$
O=\frac{1}{\pi}(t-\sin t \cos t)\left(\sec \theta_{s}^{\prime}+\sec \theta_{v}^{\prime}\right)
$$

$$
\cos t=\frac{h}{b} \frac{\sqrt{D^{2}+\left(\tan \theta_{s}^{\prime} \tan \theta_{v}^{\prime} \sin \phi\right)^{2}}}{\sec \theta_{s}^{\prime}+\sec \theta_{v}^{\prime}}
$$

$$
D=\sqrt{\tan ^{2} \theta_{s}^{\prime}+\tan ^{2} \theta_{v}^{\prime}-2 \tan \theta_{s}^{\prime} \tan \theta_{v}^{\prime} \cos \phi}
$$

$\cos \xi^{\prime}=\cos \theta_{s}^{\prime} \cos \theta_{v}^{\prime}+\sin \theta_{s}^{\prime} \sin \theta_{v}^{\prime} \cos \phi$

$$
\theta_{v}^{\prime}=\tan ^{-1}\left(\frac{b}{r} \tan \theta_{v}\right) ; \theta_{s}^{\prime}=\tan ^{-1}\left(\frac{b}{r} \tan \theta_{s}\right)
$$

The two ratios, $h / b$ and $b / r$, describe the relative height and shape of crown. In this paper, we used values of $h / b=2$ and $b / r=1$, as recommended by Lucht et al. [2000].

\subsection{Procedure Implementation}

The implementation of proposed LBF method for derivation of surface BRDF parameters involves several steps. These are:

1. Input data are taken from the MOD09A1 product (version 4) for MODIS on Terra satellite and MYD09A1 for MODIS on Aqua satellite. These are MODIS atmosphericallycorrected surface clear-sky reflectances composited over 8-day intervals with a spatial resolution of 500 meters (http://edcdaac.usgs.gov/modis/mod09a1v4.html). The MOD09A1/MYD09A1 archive contains seven spectral bands from 0.46 to $2.15 \mu \mathrm{m}$. Bands are located in the shortwave domain and designed for land monitoring applications. Data are calibrated to an absolute scale 
using the MODIS on-board calibration technique and are accurately geolocated. The dataset also includes snow and ephemeral water flags. Cloud screening and atmospheric corrections are carried out using the synchronous onboard observations available from the ensemble of MODIS channels and MODIS data processing technology [Vermote and Vermeulen, 1999].

2. The MOD09A1/MYD09A1 data are remapped using MODIS re-projection tool (http://edcdaac.usgs.gov/landdaac/tools/modis/index.asp) from the native Sinusoidal (SIN) grid onto a latitude-longitude projection over the ARM-SGP $8^{\circ} \times 10^{\circ}$ area. A pixel size of 500-meters was maintained during the re-projection procedure. To maintain compatibility with other widely used datasets, the data are then re-arranged from an 8-day composite interval to a 10-day composite interval. This procedure is possible because the MOD09A1/MYD09A1 data contain information on the data acquisition date of each pixel.

3. All pixels in the study area are grouped according to landcover type. To reduce noise, pixels marked as bad quality or cloud contaminated are excluded from the analysis. Pixels that contain some portion of snow cover are selected for separate processing. Snow pixels can be identified by their high reflectance in the visible band and relatively low reflectance in the shortwave infrared band. The normalized difference snow index (NDSI) or operational snow cover products can be used to identify and remove snow effects in surface reflectance [Hall et al., 2002].

4. The landcover-grouped data are further grouped into smaller bins according to their NDVI levels and values of angular variables. Data binning according to angular values is required to lessen the impact of the irregular distribution of the observational geometry. The histograms of VZA presented in Figure 3 show the distribution of sampling geometries for several landcover types and seasons. The distribution of angles depends on several factors, such 
as the orbit configuration, the sensor scanning mode, the distribution of the clear-sky area, and the spatial patterns of landcover. The distribution of VZA and RAA is generally non-uniform. If the data are directly used in the fitting process, unequal weights at different angles may bias the fitting results towards data with the most frequently observed geometries. This causes the equally important but less frequent data points to have little influence on the retrieved BRDF model. To address this problem, all data points for each landcover class are sorted into data bins defined by certain NDVI and angular intervals, and only one value from each data bin contributes to the fitting process. In this paper intervals for the NDVI, and the SZA, VZA and RAA, are 0.1, and $5^{\circ}, 5^{\circ}$ and $10^{\circ}$, respectively.

5. Before the fitting procedure is initiated, the data collected in each data bin are statistically pre-processed. A robust statistical procedure resistant to outliers similar to that proposed by Trishchenko [2002] is employed. Procedure sorts all data according to their values, removes the lowest and highest $5 \%$ points of the sample and then computes the average. Only one value is derived for each bin after applying the statistical procedure. This value represents the observed reflectance and geometry for that data bin. Examples of bin-processed data for grasslands are given in Figure 4 (SZA is not grouped here). Figure 4 also shows the polar plots of MODIS surface reflectance in two spectral bands (red and NIR) for four months over the ARM SGP study area. The average SZA value and its range are given at the top of each figure. The radius of the polar plot represents the VZA, and the polar angle corresponds to the RAA. The polar plots clearly show how the azimuth angle varies with season. This angle is close to the perpendicular plane $\left(90 / 270^{\circ}\right)$ in winter and closer to the principal plane $\left(0 / 180^{\circ}\right)$ in summer. The BRDF shape generally shows larger reflectances in the backscattering direction, when the RAA is close to the principal plane. This is consistent with the commonly-observed hot-spot 
effect [Kuusk, 1991]. It is important to note the narrow range of RAA,, which is a major source of uncertainty in determining the BRDF function for the entire range of 3 variables (SZA, VZA and RAA).

6. The landcover-grouped and pre-processed data are used to determine a set of optimal parameters for the Ross-Li BRDF model (Equation (1)). In this paper, the nonlinear least-squares method of Levenberg-Marquardt [Press et al., 1992] is employed for the fitting process.

7. Pixel-level BRDF parameters are retrieved by scaling the landcover- and NDVIdependent BRDF parameters obtained in step (6) to the observations at each pixel according to Equation (4):

$$
\rho_{\lambda}\left(\theta_{s}, \theta_{v}, \phi\right)=\left[1+\frac{a_{1}}{a_{0}} f_{1}\left(\theta_{s}, \theta_{v}, \phi\right)+\frac{a_{2}}{a_{0}} f_{2}\left(\theta_{s}, \theta_{v}, \phi\right)\right] \frac{\rho_{\lambda}^{o b s}\left(\bar{\theta}_{s}, \overline{\theta_{v}}, \bar{\phi}\right)}{\left[1+\frac{a_{1}}{a_{0}} f_{1}\left(\overline{\theta_{s}}, \overline{\theta_{v}}, \bar{\phi}\right)+\frac{a_{2}}{a_{0}} f_{2}\left(\overline{\theta_{s}}, \overline{\theta_{v}}, \bar{\phi}\right)\right]}
$$

where $\bar{\theta}_{s}, \bar{\theta}_{v}, \bar{\phi}$ are angles corresponding to the geometry of observed clear-sky pixel.

When the observed data for some pixels are cloud-contaminated or are of bad quality, an interpolation is performed based on the model results (derived from clear-sky data elsewhere in the region) and multi-year statistics for a particular pixel and time interval. The pixel-level BRDF parameters are temporally interpolated and smoothed if further data gaps or outliers are identified. This process uses a complete phenological cycle (12 months) of time series data for each pixel, and employs a Fourier transform filter technique.

8. The hemispheric reflectance - i.e. albedo - is computed using the BRDF parameters. Two types of albedo are distinguished: direct and diffuse [Iqball, 1983]. Direct albedo is defined as the albedo of the surface illuminated by a direct beam of radiation. Diffuse albedo is defined as the albedo of the surface under a fully isotropic downward radiance field. With a linear model like the Ross-Li, both types of albedo can be computed using a look-up table of pre-computed 
kernel integrals or by using analytical approximations that express albedo as a function of BRDF parameters and SZA [Lucht et al., 2000; Schaaf et al., 2002]. The albedo of the surface under natural illumination, i.e. the superposition of direct and diffuse radiance fields, can be estimated as a linear combination of direct and diffuse albedo components, with the weights depending on the atmospheric conditions [Lewis and Barnsley, 1994].

\section{BRDF and Albedo Results}

The results of fitting MODIS reflectances by the Ross-Li model using the LBF methodology are shown in Figure 5. The reflectances in the red and NIR bands are plotted against the VZA for grasslands, croplands, and deciduous forest at various NDVI levels for April 2001. As expected, these plots show that observations are sampled almost continuously against the VZA axis and with approximately equal weights. The observations cover a wide range of viewing geometries and clearly produce an anisotropic BRDF shape. The observations and model fitting results are distributed as tight clusters. This indicates that successful fittings have been mostly achieved. The BRDF dependence upon NDVI is smooth within each landcover type. The magnitude of a BRDF varies much more than its shape, which provides an additional support to the LBF methodology. On the other hand, the differences in the BRDF shape among the landcover types are obvious. For example, grasslands always show relatively smaller forward scattering (i.e. when $\mathrm{VZA}>0$ ) and much smoother backward scattering reflectances (i.e. when $\mathrm{VZA}<0$ ) compared to cropland. This is probably due to the smaller size of grass leaves and larger and more uniform volume density of grasslands [Wu et al., 1995]. In the case of forests, a distinct reflectance peak in the back-scattering direction is observed. This is caused by their large 
protruding canopy shape. The same argument may be made from Figure 6, that conveys similar information to that shown in Figure 5, but for July 2001,. Here, the fitting results for grasslands and croplands are close, indicating similarity of their properties in summertime. The BRDF shapes for croplands and forest in July are considerably different from those in April. This partially reflects the fact that the canopy for the croplands and forest changes differently between the summer and spring.

The retrieved parameters of the Ross-Li BRDF model for four major landcover types in April 2001 at different NDVI levels are presented in Tables 1 and 2 for the red and NIR bands, respectively. The relative errors (standard deviations normalized by the mean), which indicate the agreement between the model fit and observations, are also listed. Most errors are less than $10 \%$, which indicates successful fitting. On the other hand, one may notice a deficiency in fitting the BRDF for the NIR reflectance over cropland whose data points show apparent hot spots, but the fitting curves tend to miss them. This may originate from the limitation of the kernel functions [Chen and Cihlar, 1997].

For theoretical modeling kernels, the parameters $a_{0}, a_{1}$ and $a_{2}$ are considered as functions of the surface structural properties, the optical properties of canopy elements and the Leaf Area Index (LAI) [Wanner et al., 1995]. If it is assumed that the canopy structure and optical properties are defined by landcover type, then for a given landcover type the parameters $a_{0}, a_{1}$ and $a_{2}$ should only be a function of LAI, which is highly correlated to NDVI [Wu et al., 1995; Li et al. 1996; Knyazikhin et al., 1998]. Because two kernels in the Ross-Li model diminish to zero when $\theta_{v}=0$ and $\theta_{s}=0$, parameter $a_{0}=\rho_{\lambda}(0,0, \phi)$, which is the bi-directional reflectance at nadir view and overhead sun. 
Tables 1 and 2 show that when NDVI increases, the value of $a_{0}$ for all landcover types monotonically decreases in the red band, but increases in the NIR band. This is a typical feature of reflectance for vegetated surfaces. However, other parameters do not always display such a simple relationship with NDVI. For example, while the volumetric scattering parameter $a_{1}$ decreases for the red band and increases for the NIR band for shrublands, the geometric parameter $a_{2}$ decreases for both bands. This may imply that shrublands become denser and more uniform as NDVI increases. As a result, volumetric scattering is weakened in the red band due to a higher absorption by denser leaves. It is more intensive in the NIR band due to the greater reflection by denser leaves. The geometric scattering, caused by shadowing effects, becomes less significant with increasing NDVI. However, in the case of the forested landcover types, geometric scattering becomes more significant, and $a_{2}$ increases with NDVI, especially in the NIR band. The isotropic scatterings $\left(a_{0}\right)$ of grassland and cropland are similar at different NDVI levels (although the values for croplands are slightly larger), the volumetric scattering of grasslands is larger than that of croplands, and the geometric scattering of grasslands is generally smaller than croplands. The latter finding probably occurs because grass leaves are smaller in size and denser in volume than croplands.

Negative values of $a_{1}$ or $a_{2}$ may appear occasionally during unconstrained inversion processes, and usually when these parameters are very small. Such values correspond to unphysical behavior of the model kernels [Lucht and Roujean, 2000]. This may occur due to noise in the data and/or insufficient angular samplings. Another possible reason is the lack of orthogonality between the model kernels. Negative values for one parameter can be compensated by other parameters to make the overall results well fitted to the observations. In our case, when 
a negative solution occurs for a parameter, it is set to zero and the fitting process for other parameters is repeated.

Figure 7 shows the BRDF effects over the entire viewing domain, as provided by a model fit for the observations previously illustrated in Figure 4. The magnitude and pattern of BRDF vary with season, which reflects the change of surface properties and sun-sensor geometrical conditions. Although the highest reflectances are observed in the backward direction along the principal plane $\left(0 / 180^{\circ}\right)$, the hot-spot effect is not always well pronounced. This is partially due to the fact that the BRDF shape is retrieved from observations off the principal plane (cf. Figure 4). A model specifically designed to produce a sharp hot-spot effect has been proposed by Chen and Cihlar [1997], but we do not use it here because it introduces two additional non-linear parameters that can only be reliably retrieved by employing observations in the vicinity of the principal plane.

\section{Comparison with MODIS and MISR Albedo Products}

To assess the quality of the BRDF and albedo product derived by different algorithms i.e. the PBF and LBF methods and approaches based on different BRDF models - we compare the LBF results described earlier to the standard MODIS albedo products [MOD43; Schaaf et al., 2002] and the MISR surface products [Diner et al., 1999]. The MODIS albedo products have recently been validated against ground measurements through the up-scaling of fine-resolution satellite imagery such as Landsat ETM+ [Liang et al., 2002], and observations from other satellite systems [Jin et al., 2003b]. 


\subsection{Comparison of BRDF Kernel Parameters.}

Figures $8 \mathrm{a}$ and $8 \mathrm{c}$ show the distribution of the three kernel parameters $a_{0}, a_{1}$ and $a_{2}$ in the red and NIR bands from the PBF-derived MODIS 16-day albedo product (MOD43B1). Figures $8 \mathrm{~b}$ and $8 \mathrm{~d}$ show the same parameters, but from the LBF method. All histograms in Figure 8 are normalized with peak values equal to 1 . Most distributions have a single peak value. However, a number of the distributions are bi-modal. This indicates that the data points for most individual landcover types are closely related, while some others, such as the grasslands, may reveal the intraclass heterogeneity. That is, they can be separated into sub-classes, for example, pasture-like grasslands and grasslands containing some portion of forest or crops fields. The large fraction of zero values for the volumetric $\left(a_{1}\right)$ and geometric $\left(a_{2}\right)$ parameters from the PBF retrieval indicates frequent failures of the inversion procedure [Schaaf, et al., 2002; Jin et al., 2003a]. The LBF approach has a much higher rate of successful fitting. In general, the LBF and PBF fitting procedures show good agreement for grassland. The LBF volumetric parameter distribution is much sharper than the distribution from the MOD43 product. This is because the LBF method uses a larger number of data points for each fitting process, a shorter sampling interval of 10 days, and a higher spatial pixel resolution of 500 meters. The MOD43 product has a sampling interval of 16 days, and a spatial resolution of $1 \mathrm{~km}$. The LBF approach is thus able to reduce intrinsic noise in its data and capture finer-resolution spatial heterogeneity.

A noticeable difference between the PBF and LBF approaches occurs between the forest volumetric and geometric parameters. The PBF fails to fit the red band volumetric parameter for a significant portion of forest pixels, while the LBF successfully retrieves this parameter. Due to its frequent inability to fit the red band volumetric parameter, the PBF approach's geometric parameter is also questionable. In the NIR band, the PBF obtains significantly larger and broader 
volumetric scattering and smaller geometric scattering parameters compared to the LBF approach. Considering the forest properties in the spring season, lower volumetric but higher geometric scatterings may be expected. Thus, it appears that the LBF method provides more realistic results. In addition to the differences in spatial resolution, the above results may also be caused by significant temporal variations in surface properties during the 16-day sampling period. Numerous cases of fitting failure for the geometric parameter may lead to biases for other BRDF parameters.

\subsection{Comparison of Albedo Maps.}

Another way of assessing the agreement between the different methodologies is a direct comparison of the albedo values produced by different approaches. Figure 9 displays clear-sky albedo computed at local solar noon for the red and NIR bands over the ARM-SGP area. These images correspond to MODIS LBF albedo in bands 1 (red, Figure 9a) and 2 (NIR, Figure 9b), and to MISR albedo in bands 3 (red, Figure 9c) and 4 (NIR, Figure 9d). The LBF-derived albedo maps correspond to an average of three MODIS 10-day clear-sky albedo composites (April 1-10, 11-20, and 21-30) from 2001. The MISR-derived albedo maps correspond to a 1-month composite created by mosaicking individual MISR orbits from day 91 to 120 during 2001. These data are extracted from the MISR Level 2 Land Surface Data product, and albedo is derived using the modified Rahman's BRDF model employed in MISR data processing [Diner et al., 1999]. Albedo over the white areas in Figures 9c and 9d could not be determined due to persistent cloudiness observed in MISR data within entire time interval.

Figure 9 shows that the MODIS LBF and MISR produce surface albedo maps with similar spatial patterns and magnitude even though results are based on different methodologies 
and different sensors. However, the LBF albedo maps are much less influenced by cloud contamination and retrieval failures. This is most apparent if comparing the northwest corners of the MISR albedo images (Figures 9c and 9d). In addition, the MISR product generally produces larger albedo values than the LBF approach in the southern part of the study area. This phenomenon likely relates to the temporal mismatch between the MODIS LBF and MISR data points (an average of three 10-day composites vs. a one month composite, respectively). Detailed analysis shows that the MISR albedo data in the southern part of the image are filled mostly from observations made on April 25 (a MISR compositing uses 1 point during a month). As a result, the noted discrepancy between datasets is caused by the rapid changes of surface vegetation properties in the spring season. This is also illustrated in Figure 10, which shows three individual MODIS LBF 10-day data products (band 2, NIR) for the southeastern portion of the study area $\left(32-38^{\circ} \mathrm{N}, 92-98^{\circ} \mathrm{W}\right)$. These images clearly show significant changes in surface albedo in springtime. Figure 10 also re-emphasizes the advantage of the LBF approach for mapping rapid changes in surface properties over short-time intervals.

Pixel-by-pixel comparisons between MISR and MODIS LBF albedo in the red and NIR bands are presented in Figure 11 for April 2001. Results are grouped into the five major landcover types identified in the ARM SGP area. In most cases the data points are distributed around the 1:1 line. Correlation coefficients $(R)$ between the two data sets vary from 0.55 to 0.92 (mostly greater than 0.8 ). The differences in spatial resolution between MISR and MODIS images and resampling during reprojection step may contribute to scattering of the points and toward reducing the correlation coefficient. The mean bias $(\bar{\Delta})$ is also shown in Figure 11. It is an average of differences between the MISR and MODIS albedos. Positive $\bar{\Delta}$ means MISR albedo is greater than MODIS on average. Both $R$ and $\bar{\Delta}$ are indicators of any systematic 
discrepancies that may exist between the two data sets. Mean biases are small. The absolute values of mean biases on Figure 11 are less than 0.01 for the red band and less than 0.037 for the NIR band. Another important parameter also shown in Figure 11 is the standard deviation $(\delta)$ of the bias. The values of $\delta$ are of magnitude around 0.01 for the red band and 0.02 for the NIR band. In general, MISR albedo is slightly larger than MODIS albedo. Such bias may be caused by many factors, such as differences in calibration, atmospheric corrections, and spectral response functions of the MODIS and MISR instruments [Trishchenko et al., 2002]. This bias is also consistent with the findings of Kahn et al., [2004].

Among all landcover types, grasslands show the best correlation between sensors, while the evergreen needleleaf forest shows less correlations. These differences can be explained, at least partially, by the difference in BRDF models used. The RossThickLiSparse model used for MODIS data processing is more suitable to a surface covered by shorter vegetation, while the Rahman's model used in the MISR products is more accurate for taller vegetation such as forest canopies [Privette et al., 1997].

Pixel-by-pixel scatter plots for July 2001 are shown in Figure 12. Again, good agreement is found between MODIS LBF and MISR datasets. The best correlations are found between grasslands and croplands, while the weakest correlations are found for evergreen needleleaf forest. It is worth noting that a considerable number of missing and cloud contaminated pixels are present in the MISR composite albedo map for July (not shown). Cloud contamination may also partially contribute to the observed scattering of data points. When albedo comparisons include results derived for other months, a good agreement is generally found. The average biases in Figure 12 for the red and near-infrared bands are usually less than 0.01 and 0.015 , the 
standard deviation of biases usually being less than 0.02 , and the correlation coefficients typically being larger than 0.80 .

\section{Concluding Remarks}

The objectives of this work were to: (i) develop a methodology suitable for retrieving surface BRDF/albedo properties from MODIS data available at $500 \mathrm{~m}$ spatial resolution and at 10-day time intervals; (ii) overcome the difficulties in the pixel-based fitting method that are caused by using a small or insufficient number of clear-sky data points in the derivation of BRDF parameters; and (iii) generate a spatially and temporally complete BRDF/albedo product over the ARM SGP area. To achieve these objectives, a landcover-based fitting (LBF) method was proposed, and applied to MODIS 500m clear-sky directional reflectance data. Clear-sky albedo composites for 10-day intervals were derived from the MOD09A1/MYD09A1 surface reflectance product. The RossThick-LiSparse model was used to describe the shape of BRDF. A robust statistical preprocessing was implemented to provide input for the model inversion. All observations were divided into data bins according to solar zenith angle (SZA), view zenith angle (VZA) and sun-satellite relative azimuth angle (RAA) for a given landcover type and NDVI range. A single statistical value for each data bin was produced using robust estimation. The BRDF model parameters were derived through the fitting procedure.

The proposed LBF approach has several advantages. First, it increases the size of samples used in the BRDF fitting procedure, thus making the retrieval of BRDF shapes more reliable. Second, it allows the implementation of a pre-processing procedure that performs data binning, which serves to reduce noise and prevent bias caused by an uneven distribution of observational geometries. Third, it is often the only available method for producing surface BRDF/Albedo products from historical datasets, which are commonly only available as a multi-day clear-sky 
composite. Fourth, LBF-derived BRDF and albedo products may be suitable for direct applications in the studies of the regional phenological cycle and land surface modeling. Landcover-based schemes are often used to parameterize surface albedo properties in climate and GCM modeling.

While the LBF approach complements the pixel-based fitting (PBF) strategy currently employed in the derivation of the MODIS standard BRDF/Albedo product (MOD43), it has some advantages over the standard (PBF-derived) product. These include higher spatial resolution, shorter sampling interval, higher successful fitting rate, and capability to produce spatially complete product. It performs better and generates more realistic results when the surface vegetation state changes quickly and number of clear-sky pixels are limited due to frequent cloudiness.

The methodology presented in this paper is rather general. It can be applied to other regions and data from other sensors. Because our approach is based on landcover type sampling instead of pixel-based sampling, it can easily be applied to the data processing of multi-day clear-sky composite data assembled from multiple platforms, such as Terra/MODIS, Aqua/MODIS, SPOT/VEGETATION and NOAA/AVHRR. A spectral correction procedure must be implemented to merge data from similar but not identical sensors to account for the differences in their spectral responses [Trishchenko et al., 2002]. With such an approach, a more comprehensive multi-satellite surface product can be generated.

A good agreement between MISR-derived and LBF-derived albedo was found. Correlations between these datasets are usually larger than 0.80 , and mean biases and their standard deviations are typically below 0.01 and 0.02 . The distribution of model parameters 
derived using LBF approach also compares reasonably well with standard MOD43 product. It demonstrates more realistic features and higher success rates of model inversion.

Acknowledgements. Authors are grateful to C. Schaaf for help with MODIS BRDF/albedo data and G. Schmidt for help with the MODIS re-projection tool. Authors are also thankful to their colleagues, A. Davidson and D. Pouliot, for their help on editing the manuscript. MODIS and MISR data were acquired through the NASA Distributed Active Archive Center (DAAC). This research was supported by the US Department of Energy Atmospheric Radiation Measurement (ARM) Program under grant No. DE-FG02-02ER63351 and DEFG0201ER63166. 


\section{References}

Ackerman, T.P., and G.M. Stokes (2003), The Atmospheric Radiation Measurement Program, Physics Today, 56(1), 38-43.

Chen, J., and J. Cihlar (1997), A hot spot function in a simple bidirectional reflectance model for satellite applications, J. Geophys. Res., 102, 25,907-25,913.

Cihlar, J., R.Latifovic, J.Chen, A.Trishchenko, Y.Du, G.Fedosejevs, G.Guindon (2004),

Systematic corrections of the AVHRR image composites for temporal studies. Remote Sens.

Environ., 89, 217-233.Cihlar, J., J. Chen, Z. Li, R. Latifovic, G. Fedosejevs, M. Adair, W.

Park, R. Fraser, A. Trishchenko, B. Guindon, D. Stanley, and D. Morse (2002), GeoComp-n, an advanced system for the processing of coarse and medium resolution satellite data. Part 2:

Biophysical products for northern ecosystems, Can. J. Remote Sens., 28, 21-44.

Csiszar, I., G. Gutman, P. Romanov, M. Leroy, and O. Hautecoeur (2001), Using

ADEOS/POLDER data to reduce angular variability of NOAA AVHRR reflectances, Remote Sens. Environ., 76, 399-409.

Diner, D.J., J.C., Beckert, T. H. Reilly, C. J. Bruegge, J. E. Conel, R. A. Kahn, J. V. Martonchik, T.P. Ackerman, R. Davies, S. A. W. Gerstl, H. R. Gordon, J.-P. Muller, R. B. Myneni, P. J.

Sellers, B. Pinty, M. M. Verstraete, (1998), Multi-angle Imaging SpectroRadiometer (MISR)

Instrument Description and Experiment Overview. IEEE Trans. on Geosci and Remote Sensing. 36,. 1072-1087.

Diner, D.J., J.V. Martonchik, C. Borel, S.A.W. Gerstl, H.R. Gordon, Y. Knyazikhin, R. Myneni, B. Pinty, and M. Verstraete (1999), MISR level 2 surface retrieval, Report of JPL D-11401 (http://eospso.gsfc.nasa.gov/eos_homepage/for_scientists/atbd/docs/MISR/atbd-misr-10.pdf).

Dirmeyer, P.A., and J. Shukla (1994), Albedo as a modulator of climate response to tropical deforestation, J. Geophys. Res., 99, 20,863-20,878.

Gao, F., Y. Jin, X. Li, C.B. Schaaf, and A.H. Strahler (2002), Bidirectional NDVI and atmospherically resistant BRDF inversion for vegetation canopy, IEEE Trans. Geosci. Remote Sens., 40, 1,269-1,278.

Gutman, G. (1987), The derivation of vegetation indices from AVHRR data, Int. J. Remote Sens., $8,1235-1243$. 
Gutman, G., A. Gruber, D. Tarpley, and R. Taylor (1989), Application of angular models to AVHRR data for determination of the clear-sky planetary albedo over land surfaces, $J$. Geophys. Res., 94, 9959-9970.

Hall, D.K., G.A. Riggs, V.V. Salomonson, N. DiGiromamo, and K.J. Bayr (2002), MODIS Snow-Cover Products, Remote Sens. Environ., 83, 181-194.

Houghton, J.T., Y. Ding, D. J. Griggs, M. Noguer, P. J. van der Linden, X. Dai, K. Maskell, and C.A. Johnson (Eds.) (2001), Climate Change 2001: The Scientific Basis. Contribution of Working Group I to the Third Assessment Report of the Intergovernmental Panel on Climate Change, Cambridge University Press, New York.

Ingram, W.J., C.A. Wilson, and J.F.B. Mitchell (1989), Modelling climate change: An assessment of sea ice and surface albedo feedbacks, J. Geophys. Res., 94, 8609-8622. Iqball, M. (1983), An introduction to solar radiation, Academic Press, New York, 390pp. Jin, Y., C. Schaaf, F. Gao, X. Li, A. Strahler, C. Bruegge, and J. Martonchik (2002), Improving MODIS Surface BRDF/Albedo Retrieval with MISR Multi-angle Observations, IEEE Trans. Geosci. Remote Sens., 40, 1,593-1,604.

Jin, Y., C.B. Schaaf, F. Gao, X. Li, A.H. Strahler, W. Lucht, and S. Liang (2003a), Consistency of MODIS surface bidirectional reflectance distribution function and albedo retrievals: 1 . Algorithm performance, J. Geophys. Res., 108(D5), ACL 2-1 to ACL 2-13.

Jin, Y., C.B. Schaaf, C.E. Woodcock, F. Gao, X. Li, A.H. Strahler, W. Lucht, and S. Liang (2003b), Consistency of MODIS surface bidirectional reflectance distribution function and albedo retrievals: 2. Validation. J. Geophys. Res., 108(D5), ACL 3-1 to ACL 3-15.

Kahn, R., W.-H.Li, J.V.Martonchik, C.J.Bruegge,D.J.Diner, B.J.Gaitley, W.Abdou, O.Dubovik, B.Holben, A.Smirnov, Z.Jin, D.Clark. (2004) MISR Low-Light-Level Calibration, and Implications for Aerosol Retrieval Over Dark Water. Journal of Atmospheric Sciences. Submitted.

Knyazikhin, Y., J.V. Martonchik, R.B. Myneni, D.J. Diner, and S.W. Running (1998), Synergistic algorithm for estimating vegetation canopy leaf area index and fraction of absorbed photosynthetically active radiation from MODIS and MISR data, J. Geophys. Res., $103,32,257-32,276$.

Kuusk, A. (1991), The hot spot effect in plant canopy reflectance, in Photon-Vegetation Interactions, edited by R. B. Myneni and J. Ross, pp.139-158, Springer-Verlag Press, Berlin. 
Latifovic R., J. Cihlar, and J. Chen (2003), A comparison of BRDF models for the normalization of satellite optical data to a standard sun-target-sensor geometry, IEEE Trans. Geosci. Remote Sens., 41, 1889-1898.

Latifovic, R., Z.-L. Zhu, J. Cihlar, C. Giri, and I. Olthof (2004), Land cover mapping of North and Central America - Global Land Cover 2000. Remote Sens. Environ., 90, 116-127

Lewis, P. and M.J. Barnsley (1994), Influence of the sky radiance distribution on various formulations of the earth surface albedo, Proc. Conf. Phys. Meas Sign. Remote Sen., Val d'Isere, France, 707-715.

Li, X., and A.H. Strahler (1992), Geometric-optical bidirectional reflectance modeling of the discrete crown vegetation canopy: Effect of crown shape and mutual shadowing, IEEE Trans. Geosci. Remote Sens., 30, 276-292.

Li, Z., M.C.Cribb, and A.P.Trishchenko (2002), Impact of surface inhomogeneity on solar radiative transfer under overcast conditions, J. Geophys. Res., 107(D16), AAC 6-1 to AAC 615, 2001JD000976.

Li, Z., J. Cihlar, X. Zhang, L. Moreau, L. Hung (1996), The bidirectional effect in AVHRR measurements over boreal regions, IEEE Tran. Geosci. \& Rem. Sen., 34, 1308-1322.

Li, Z., L. Moreau, J. Cihlar, and D.W. Deering (1997), An evaluation of kernel-driven bidirectional models using PARABOLA measurements, Can. J. Remote Sens., 23, 120-130.

Liang, S., H. Fang, M. Chen, C.J. Shuey, C. Walthall, C., Daughtry, J. Morisette, C. Schaaf, and A. Strahler (2002), Validating MODIS land surface reflectance and albedo products: methods and preliminary results, Remote Sens. Environ., 83, 149-162.

Lucht, W., C.B. Schaaf, and A.H. Strahler (2000), An algorithm for the retrieval of albedo from space using semiempirical BRDF models, IEEE Trans. Geosci. Remote Sens., 38, 977-998.

Lucht, W., and J.-L. Roujean (2000), Considerations in the parametric modeling of BRDF and albedo from multiangular satellite sensor observations, Remote Sens. Reviews, 18, 343-379.

Lucht, W., and P. Lewis (2000), Theoretical noise sensitivity of BRDF and albedo retrieval from the EOS-MODIS and MISR sensors with respect to angular sampling, Int. J. Remote Sens., 21, $81-98$.

Martonchik, J.V. (1997), Determination of aerosol optical depth and land surface directional reflectances using multi-angle imagery, J. Geophys. Res., 102, 17,015-17,022. 
Moody, E., M.D. King, S. Platnick, C.B. Schaaf, and F. Gao (2004), Spatially complete global surface albedos: value-added datasets derived from Terra MODIS land products, IEEE Trans. Geosci. Remote Sens., submitted.

Nicodemus, F.E., J.C. Richmond, J.J. Hsia, I. Ginsberg, and T. Limperis (1977), Geometric considerations and nomenclature for reflectance, U.S. Dept. of Commerce, NBS Monograph.

Press, W.H., S.A. Teukolsky, W.T. Vetterling, and B.P. Flannery (1992), Numerical Recipes in FORTRAN: The Art of Scientific Computing, $2^{\text {nd }}$ Ed. (pp.675-683), Cambridge University Press, Cambridge.

Privette, J.L., T.F. Eck, and D.W. Deering (1997), Estimating spectral albedo and nadir reflectance through inversion of simple BRDF models with AVHRR/MODIS-like data, $J$. Geophys. Res., 102, 29,529-29,542.

Rahman, H., B. Pinty, and M. Verstraete (1993), Coupled surface atmosphere reflectance (CSAR) model, 2, Semiempirical surface model usable with NOAA advanced very high resolution radiometer data, J. Geophys. Res., 98, 20,791-20,801.

Ross, J. (1981), The radiation regime and architecture of plant stands, Dr. W. Junk Publishers, Hague-Boston-London, 391pp.

Roujean, J.-L., M. Leroy, and P.-Y. Deschamps (1992), A bidirectional reflectance model of the Earth's surface for the correction of remote sensing data, J. Geophys. Res., 97, 20,455-20,468.

Schaaf, C.B., F. Gao, A.H. Strahler, W. Lucht, X. Li, T. Tsang, N.C. Strugnell, X. Zhang, Y. Jin, J.-P. Muller, P. Lewis, M. Barnsley, P. Hobson, M. Disney, G. Roberts, M. Dunderdale, C. Doll, R. d'Entremont, B. Hu, S. Liang, and J.L. Privette (2002), First operational BRDF, albedo and nadir reflectance products from MODIS, Remote Sens. Environ., 83, 135-148.

Stockes, G.M., and S. Schwartz (1994), The Atmospheric Radiation Measurement (ARM) program: Programmatic background and design of cloud and radiation test bed, Bulletin American Meteorol. Soc., 75, 1201-1221.

Strahler, A., D. Muchoney, J. Borak, M. Friedl, S. Gopal, E. Lambin, and A. Moody (1999), MODIS land cover product: Algorithm theoretical basis document (ATBD) Version 5.0, (http://modis.gsfc.nasa.gov/data/atbd/atbd_mod12.pdf).

Strugnell, N.C., W. Lucht, and C.B. Schaaf (2001), A global albedo data set derived from AVHRR data for use in climate simulations, Geophys. Res. Lett., 28, 191-194. 
Trishchenko, A.P. (2002), Removing unwanted fluctuations in the AVHRR thermal calibration data using robust techniques, J. Atmos. Oceanic Technol., 19, 1939-1954.

Trishchenko, A.P., J. Cihlar, and Z. Li (2002), Effects of spectral response function on the surface reflectance and NDVI measured with moderate resolution sensors, Remote Sens. Environ., 81, 1-18.

Trishchenko, A.P., K.Khlopenkov, Y.Luo (2004), Retrieval of BRDF for pure landcover types from MODIS and MISR using angular unmixing approach. Proc. SPIE 5549. In press.

Vermote, E.F., and A. Vermeulen (1999), Atmospheric correction algorithm: Spectral reflectances (MOD09). MODIS Algorithm Technical Background Document. Dept. of Geography, University of Maryland.

Wanner, W., X. Li, and A.H. Strahler (1995), On the derivation of kernels for kernal-driven models of bi-directional reflectance, J. Geophys. Res., 100, 21,077-21,090.

Wanner, W., A.H. Strahler, B. Hu, P. Lewis, J.-P. Muller, X. Li, C.B. Schaaf, and M.J. Barnsley (1997), Global retrieval of bidirectional reflectance and albedo over land from EOS MODIS and MISR data: Theory and algorithm, J. Geophys. Res., 102, 17,143-17,161.

Wu, A., Z. Li, and J. Cihlar (1995), Effects of land cover type and greenness on advanced very high resolution radiometer bidirectional reflectances: Analysis and removal, J. Geophys. Res., 100, 9,179-9,192. 


\section{Figure Captions}

Figure 1. Landcover map in the IGBP legend for the ARM SGP area (North Oklahoma, USA). There are total 10 landcover types identified in this area. The cross-symbol at the center indicates the location of the ARM central facility $(\mathrm{CF})$, and the squares mark the extended facilities (EF) locations.

Figure 2. (a) View of the ARM SGP CF area from the Landsat-7 ETM on day 68 (March 9) 2002. The cropland sampling square corresponds roughly to a $2.2 \times 2.2 \mathrm{~km}$ area (2x2 MISR pixels); (b) the relationship between RAA and VZA, and NDVI from MISR observations on day 68 of 2002 for the cropland sampling square; (c) the relationship between surface reflectances and VZA in the red band on day 68 of 2002; (d) the relationship between surface reflectances and VZA in the NIR band on day 68 of 2002; (e) the relationship between RAA and VZA, and NDVI from MISR observations on day 91 of 2002 for the cropland sampling square; (f) the relationship between surface reflectances and VZA in the red band on day 91 of 2002; (g) the relationship between surface reflectances and VZA in the NIR band on day 91 of 2002 . The data for the $2 \times 2$ pixels in the MISR scan are denoted by diamonds, crosses, triangles and squares as shown in panel (a).

Figure 3. Histograms of the VZA from MODIS observations for grasslands, croplands, and mixed forest (from top to bottom) in January, April, July and October of 2001 (from left to right) in the ARM SGP area. 
Figure 4. Polar plots of the surface reflectance from MODIS observations for two spectral bands (red (top) and NIR (bottom)) for grasslands in January, April, July, and October 2001 in the ARM SGP area. The SZA range, its average for each month, and NDVI intervals are indicated at the top of each panel. The radius of the polar plot represents the VZA, while the polar angle represents the RAA.

Figure 5. MODIS reflectances at the red and NIR bands against the VZA for different NDVI intervals. The main landcover types (grasslands, croplands and deciduous forest) for April 2001 are displayed. Black squares correspond to the observed values from each pre-processed data-bin (obtained in step 5, see text for details). The red symbols correspond to the results of fitting the RossThick-LiSparse BRDF model as described in the text.

Figure 6. MODIS reflectances for the red and NIR bands against the VZA for different NDVI intervals. The main landcover types (grasslands, croplands and deciduous forest) for July 2001 are displayed. Black squares correspond to the observed values from each pre-processed data-bin. The red symbols correspond to the results of fitting the RossThick-LiSparse BRDF model.

Figure 7. Polar plots of grassland surface reflectance from the RossThick-LiSparse BRDF model results based on the MODIS observations shown in Figure 4. The top panels correspond to observations in red band and bottom panels correspond to observations in the NIR band. Each column of plots corresponds to a different sampling date (from left to right: January, April, July, and October 2001). The SZA and the NDVI intervals are indicated at the top of each plot. 
Figure 8. (a) Histograms of the three RossThick-LiSparse BRDF model parameters (from top to bottom) in the red and NIR bands (left and right) from the MOD43B1 product (pixel-based fitting approach) for grasslands in April 2001; (b) BRDF parameters derived from the landcoverbased fitting algorithm for grasslands in April 2001; (c) same as (a) but for deciduous forest; (d) same as (b) but for deciduous forest.

Figure 9. Albedo images in the red and NIR bands over the ARM-SGP area. The two imagers on the left are produced using the LBF algorithm. The right two images correspond to the MISR L2 pixel-based albedo products. The MODIS image is an average of three 10-day interval albedo images for April 2001. The MISR image is a 1-month composite generated between days 91 to 120 (April) of 2001.

Figure 10. Albedo images in the NIR band for the southeast corner of Figure 9. Images are derived by the MODIS LBF algorithm for three 10-day consecutive intervals in April 2001.

Figure 11. Scatter plots of MISR albedo against MODIS albedo derived by the landcover-based fitting algorithm for five major landcover types in April 2001.

Figure12. Scatter plots of MISR albedo against MODIS albedo derived by the landcover-based fitting algorithm for five major landcover types in July 2001. 
Table 1 RossThick-LiSparse BRDF parameters for the red band (April 2001)

\begin{tabular}{|c|c|c|c|c|c|c|c|c|c|}
\hline \multicolumn{2}{|c|}{ NDVI } & $0.1-0.2$ & $0.2-0.3$ & $0.3-0.4$ & $0.4-0.5$ & $0.5-0.6$ & $0.6-0.7$ & $0.7-0.8$ & $0.8-1.0$ \\
\hline \multirow{4}{*}{ 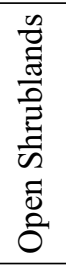 } & $\mathrm{a}_{0}$ & - & 0.171 & 0.142 & 0.118 & 0.097 & 0.076 & 0.056 & 0.042 \\
\hline & $a_{1}$ & - & 0.128 & 0.120 & 0.122 & 0.086 & 0.042 & 0.025 & 0.020 \\
\hline & $\mathrm{a}_{2}$ & - & 0.028 & 0.026 & 0.019 & 0.017 & 0.009 & 0.004 & 0.003 \\
\hline & error (\%) & - & 11.65 & 11.38 & 11.52 & 8.41 & 7.25 & 7.03 & 5.62 \\
\hline \multirow{4}{*}{$\begin{array}{l}\frac{n}{0} \\
\vec{\Xi} \\
\vec{w} \\
\tilde{\Xi} \\
\tilde{U}\end{array}$} & $\mathrm{a}_{0}$ & - & 0.150 & 0.131 & 0.107 & 0.091 & 0.075 & 0.057 & 0.043 \\
\hline & $a_{1}$ & - & 0.121 & 0.114 & 0.112 & 0.079 & 0.024 & 0.027 & 0.032 \\
\hline & $\mathrm{a}_{2}$ & - & 0.017 & 0.015 & 0.005 & 0.006 & 0.006 & 0.003 & 0.003 \\
\hline & error $(\%)$ & - & 9.43 & 9.59 & 8.13 & 6.93 & 9.20 & 7.60 & 7.17 \\
\hline \multirow{4}{*}{$\begin{array}{l}\frac{n}{0} \\
\frac{\overrightarrow{0}}{2} \\
\stackrel{0}{0}\end{array}$} & $\mathrm{a}_{0}$ & - & 0.162 & 0.140 & 0.122 & 0.104 & 0.083 & 0.061 & 0.041 \\
\hline & $a_{1}$ & - & 0.070 & 0.054 & 0.056 & 0.044 & 0.034 & 0.040 & 0.023 \\
\hline & $\mathrm{a}_{2}$ & - & 0.021 & 0.015 & 0.012 & 0.012 & 0.009 & 0.003 & 0.001 \\
\hline & error (\%) & - & 8.07 & 8.09 & 7.74 & 9.86 & 10.03 & 11.40 & 11.58 \\
\hline \multirow{4}{*}{ 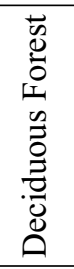 } & $\mathrm{a}_{0}$ & - & - & 0.111 & 0.101 & 0.085 & 0.070 & 0.055 & 0.042 \\
\hline & $a_{1}$ & - & - & 0.063 & 0.032 & 0.035 & 0.016 & 0.021 & 0.018 \\
\hline & $\mathrm{a}_{2}$ & - & - & 0.011 & 0.018 & 0.017 & 0.014 & 0.013 & 0.012 \\
\hline & error (\%) & - & - & 11.92 & 5.80 & 6.84 & 7.55 & 8.92 & 3.94 \\
\hline
\end{tabular}


Table 2 RossThick-LiSparse BRDF parameters for the NIR band (April 2001)

\begin{tabular}{|c|c|c|c|c|c|c|c|c|c|}
\hline \multicolumn{2}{|c|}{ NDVI } & $0.1-0.2$ & $0.2-0.3$ & $0.3-0.4$ & $0.4-0.5$ & $0.5-0.6$ & $0.6-0.7$ & $0.7-0.8$ & $0.8-1.0$ \\
\hline \multirow{4}{*}{ 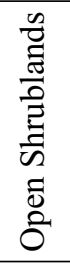 } & $a_{0}$ & - & 0.300 & 0.304 & 0.294 & 0.314 & 0.345 & 0.349 & 0.418 \\
\hline & $a_{1}$ & - & 0.219 & 0.263 & 0.365 & 0.368 & 0.259 & 0.381 & 0.247 \\
\hline & $\mathrm{a}_{2}$ & - & 0.051 & 0.057 & 0.036 & 0.041 & 0.040 & 0.027 & 0.032 \\
\hline & error (\%) & - & 11.03 & 10.52 & 11.01 & 9.87 & 5.25 & 9.16 & 4.67 \\
\hline \multirow{4}{*}{ 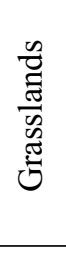 } & $\mathrm{a}_{0}$ & - & 0.262 & 0.267 & 0.282 & 0.316 & 0.342 & 0.383 & 0.437 \\
\hline & $a_{1}$ & - & 0.184 & 0.264 & 0.294 & 0.265 & 0.173 & 0.209 & 0.350 \\
\hline & $\mathrm{a}_{2}$ & - & 0.035 & 0.025 & 0.015 & 0.022 & 0.015 & 0.024 & 0.030 \\
\hline & error (\%) & - & 9.51 & 9.83 & 8.30 & 7.37 & 6.89 & 6.55 & 5.88 \\
\hline \multirow{4}{*}{ 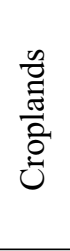 } & $\mathrm{a}_{0}$ & - & 0.275 & 0.297 & 0.321 & 0.353 & 0.379 & 0.409 & 0.445 \\
\hline & $a_{1}$ & - & 0.165 & 0.123 & 0.166 & 0.167 & 0.183 & 0.241 & 0.238 \\
\hline & $\mathrm{a}_{2}$ & - & 0.035 & 0.037 & 0.032 & 0.038 & 0.032 & 0.020 & 0.010 \\
\hline & error (\%) & - & 7.36 & 9.35 & 8.75 & 10.52 & 10.03 & 8.96 & 8.96 \\
\hline \multirow{4}{*}{ 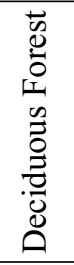 } & $\mathrm{a}_{0}$ & - & - & 0.252 & 0.260 & 0.283 & 0.315 & 0.357 & 0.395 \\
\hline & $a_{1}$ & - & - & 0.127 & 0.081 & 0.066 & 0.074 & 0.115 & 0.099 \\
\hline & $\mathrm{a}_{2}$ & - & - & 0.049 & 0.042 & 0.053 & 0.054 & 0.076 & 0.114 \\
\hline & error (\%) & - & - & 15.28 & 10.77 & 7.98 & 6.55 & 9.68 & 7.86 \\
\hline
\end{tabular}


Figure 1

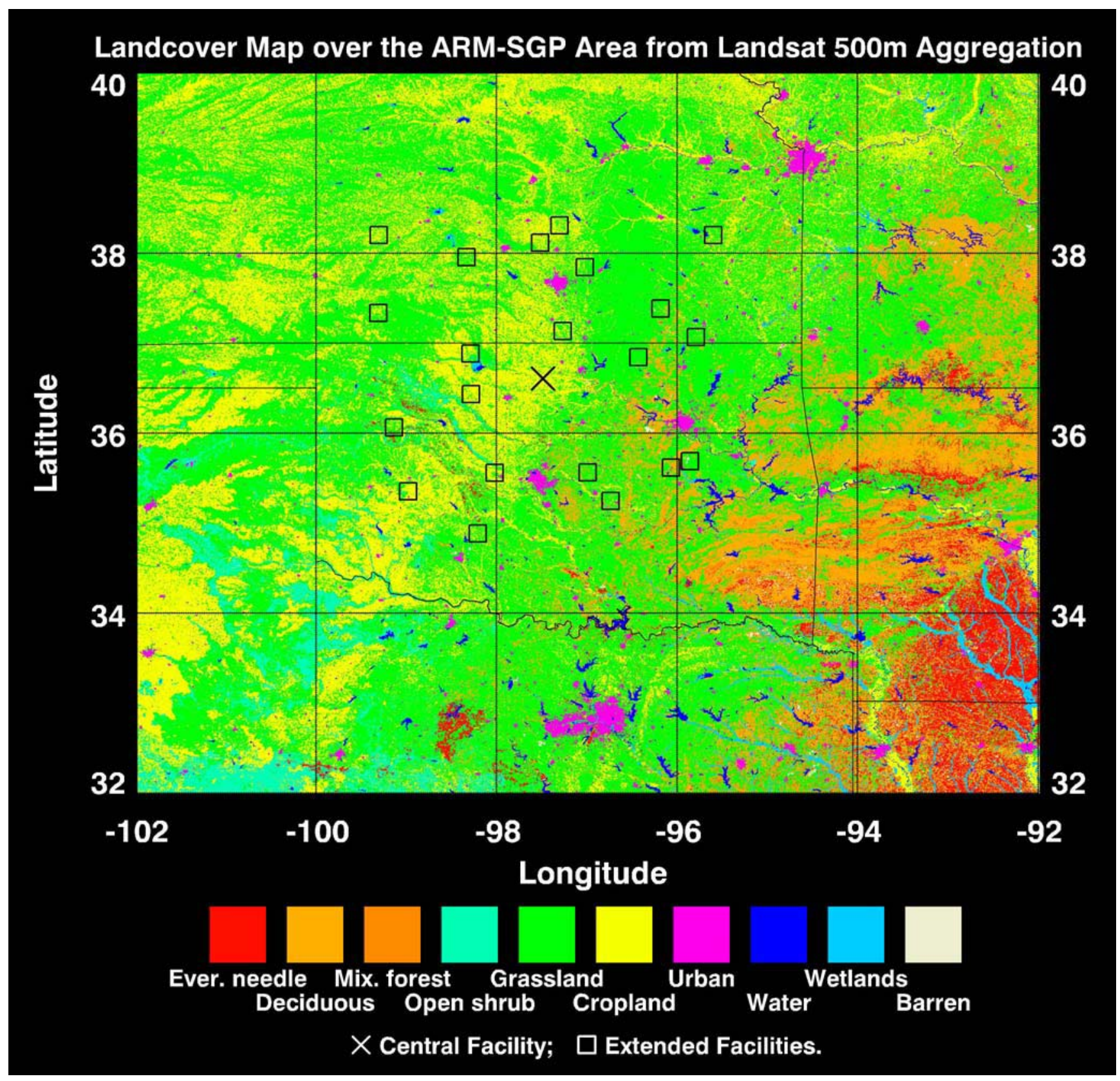


Figure 2

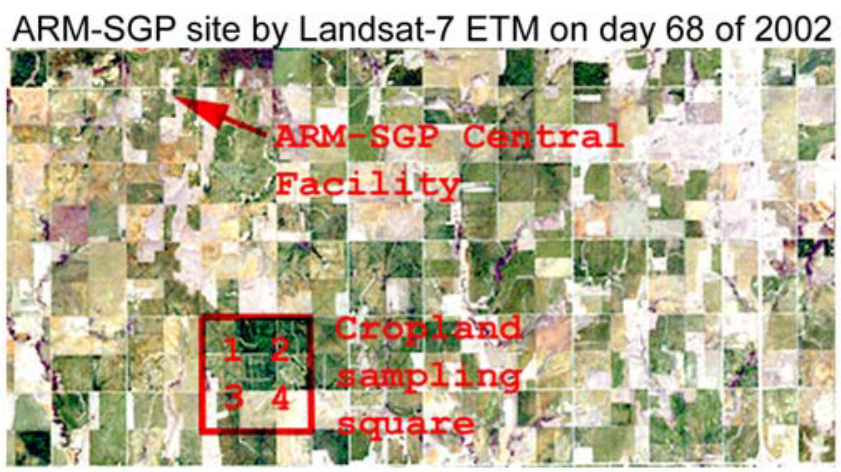

(a)

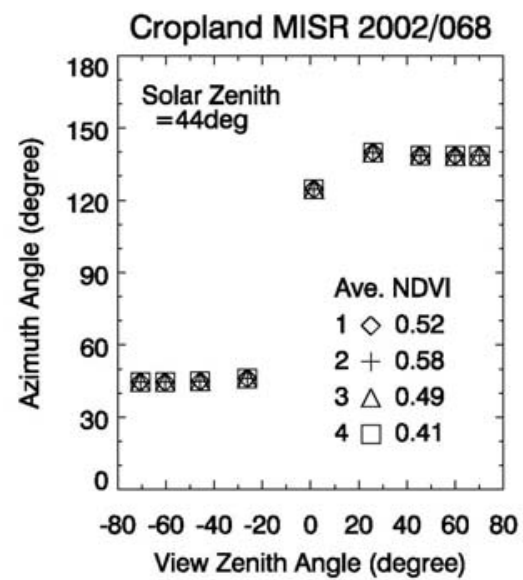

(b)

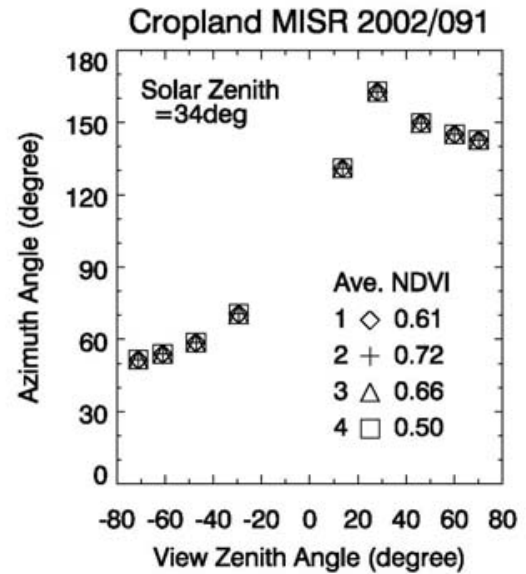

(e)

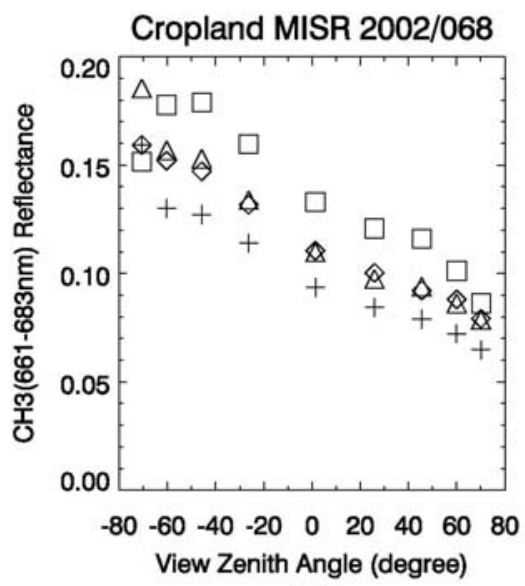

(c)

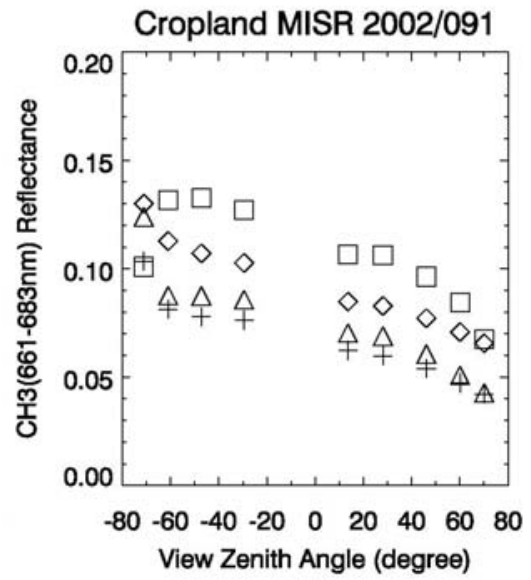

(f)

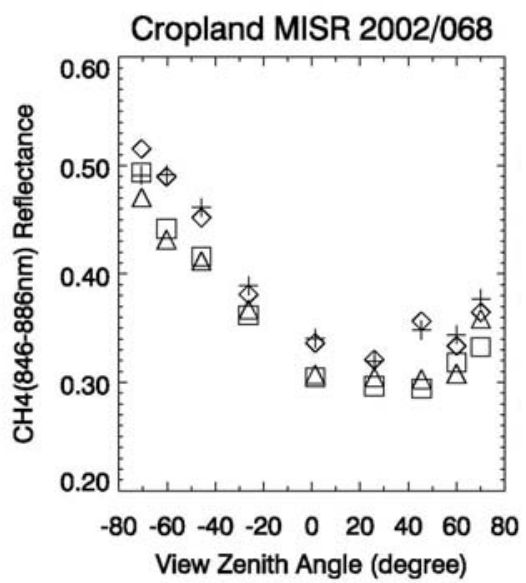

(d)

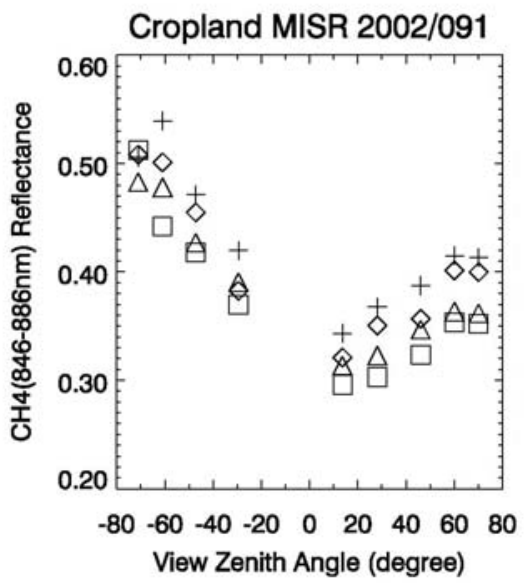

(g) 
Figure 3
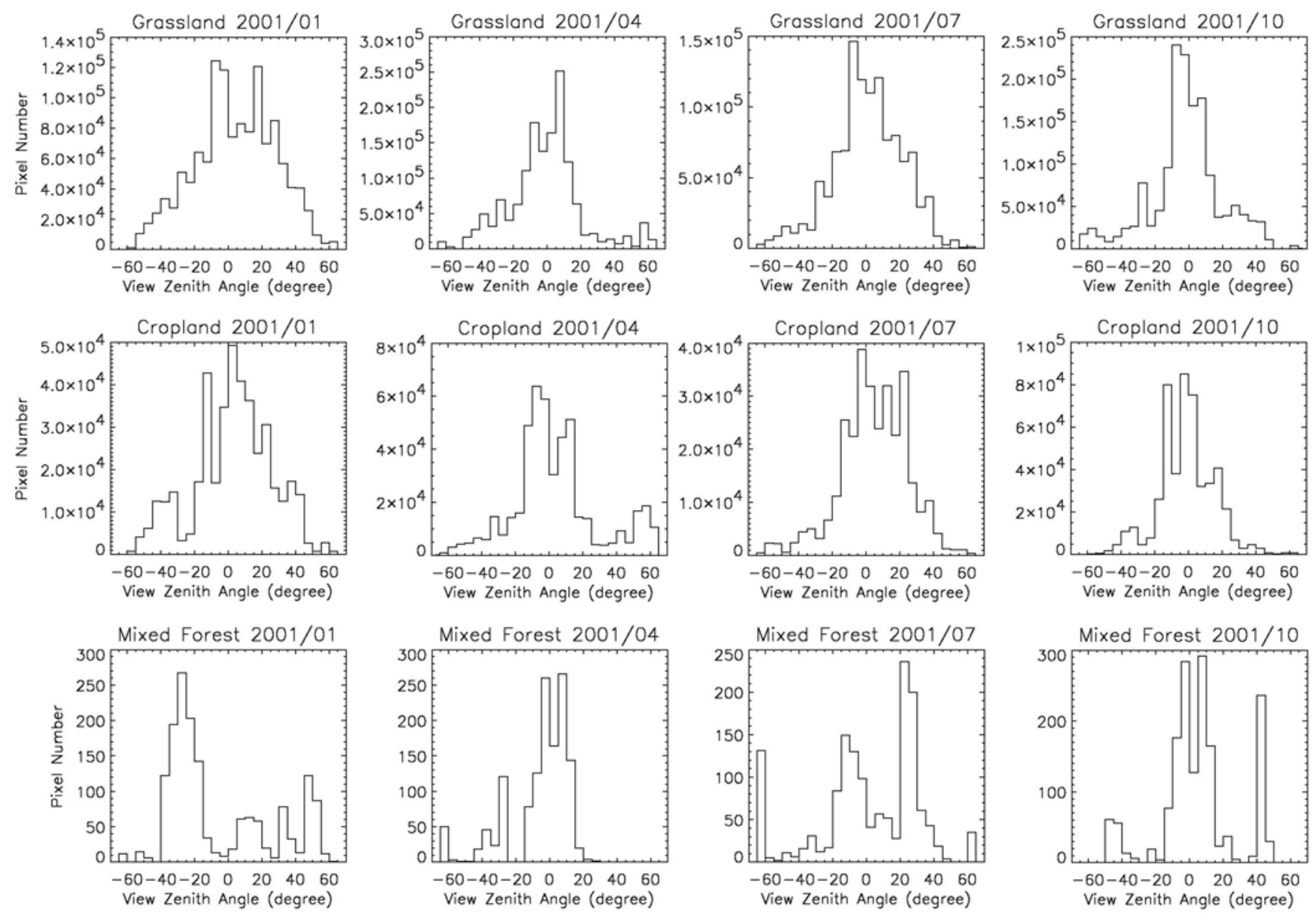
Figure 4

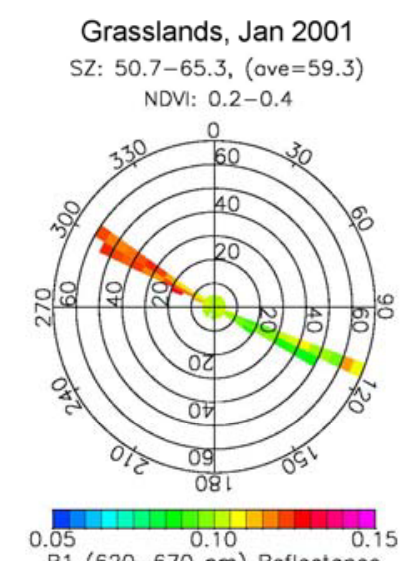

B1 $(620-670 \mathrm{~nm})$ Reflectonce
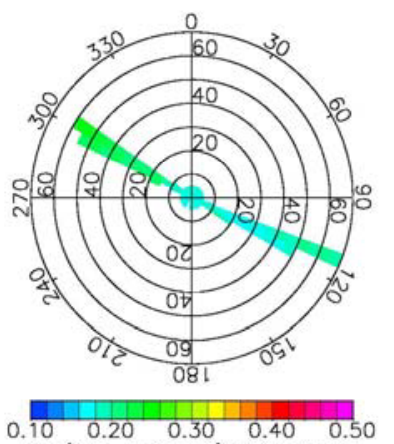

B2 $(841-876 \mathrm{~nm})$ Reflectonce
Grasslands, Apr 2001

SZ: 19.7-41.8, (ove=29.8) NDVI: $0.3-0.4$
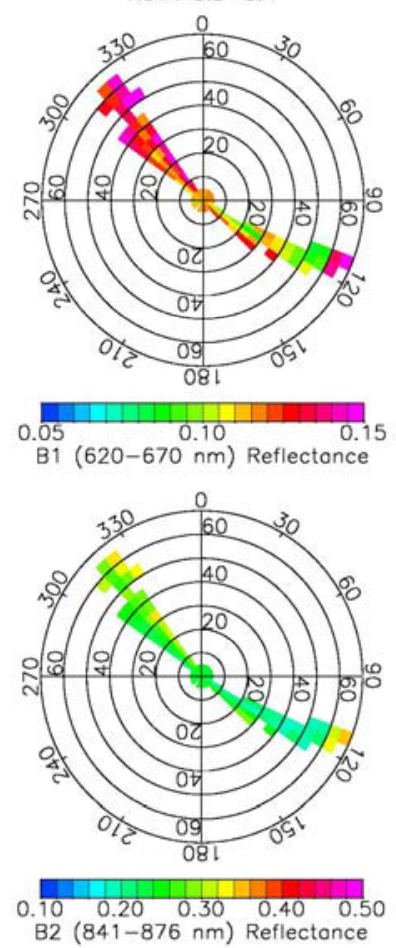

Grasslands, Jul 2001

SZ: 12.1-30.7, (ove=21.1) NDVI: $0.6-0.8$
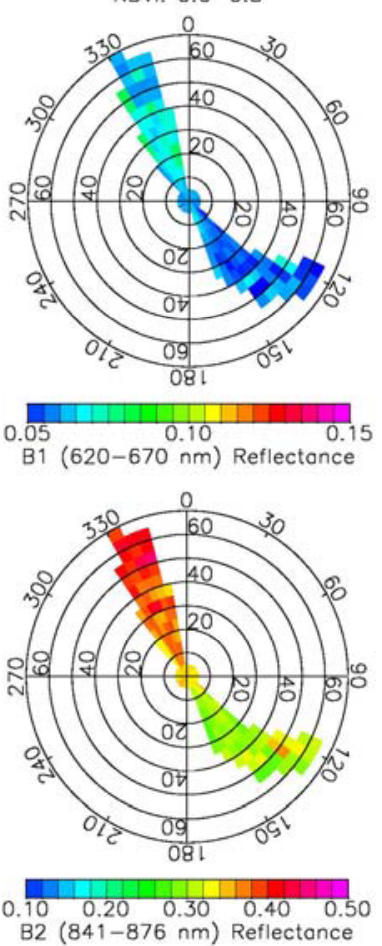

Grasslands, Oct 2001

SZ: 35.9-54.9, (ove=46.7)

NDVI: $0.4-0.6$
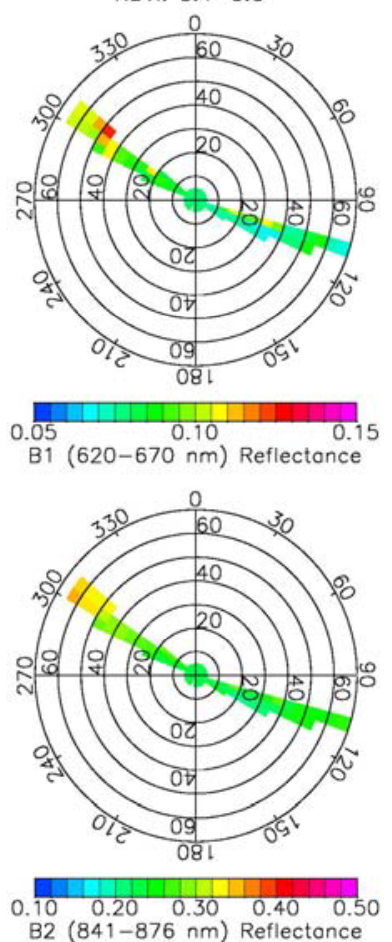
Figure 5
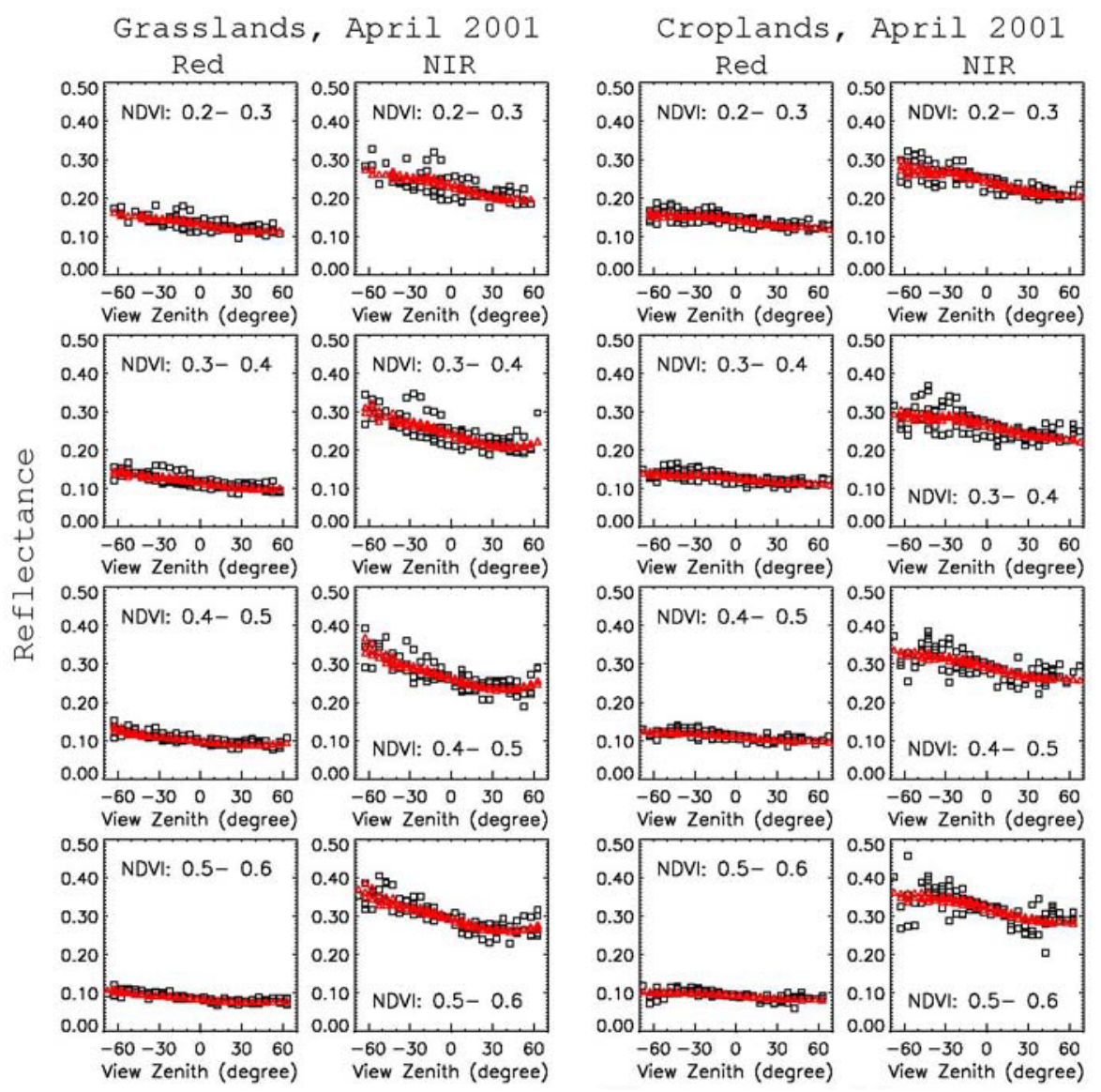

Deci. Forest, April 2001

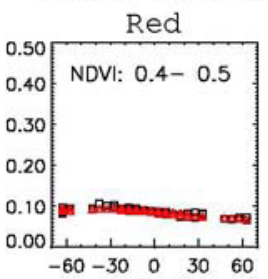

NIR
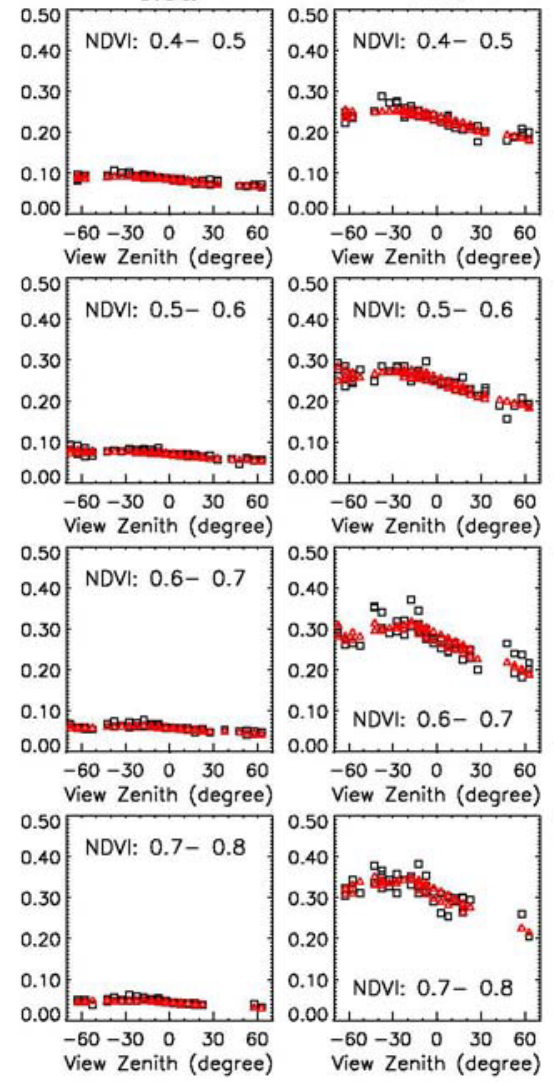
Figure 6
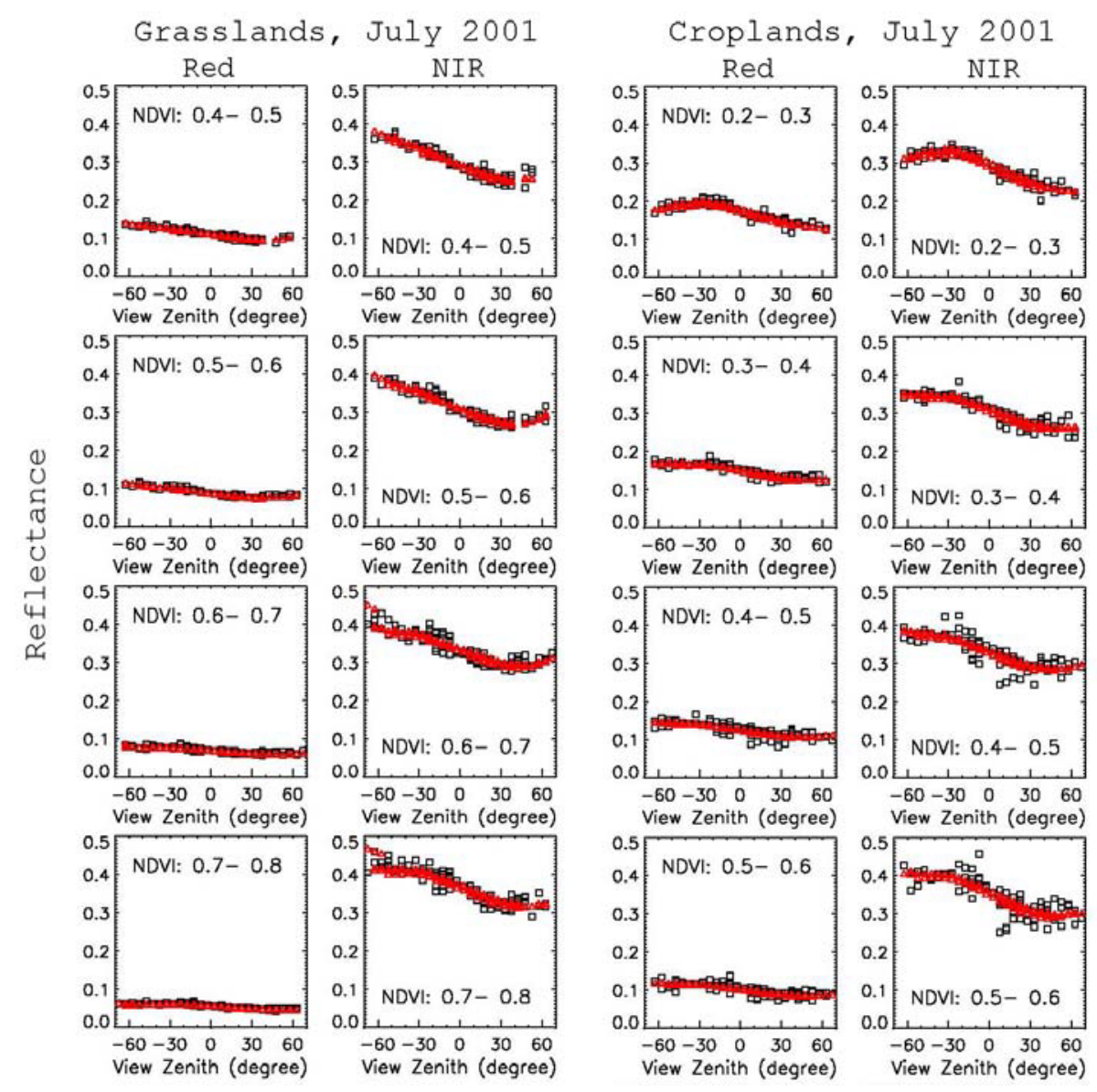

Deci. Forest, July 2001
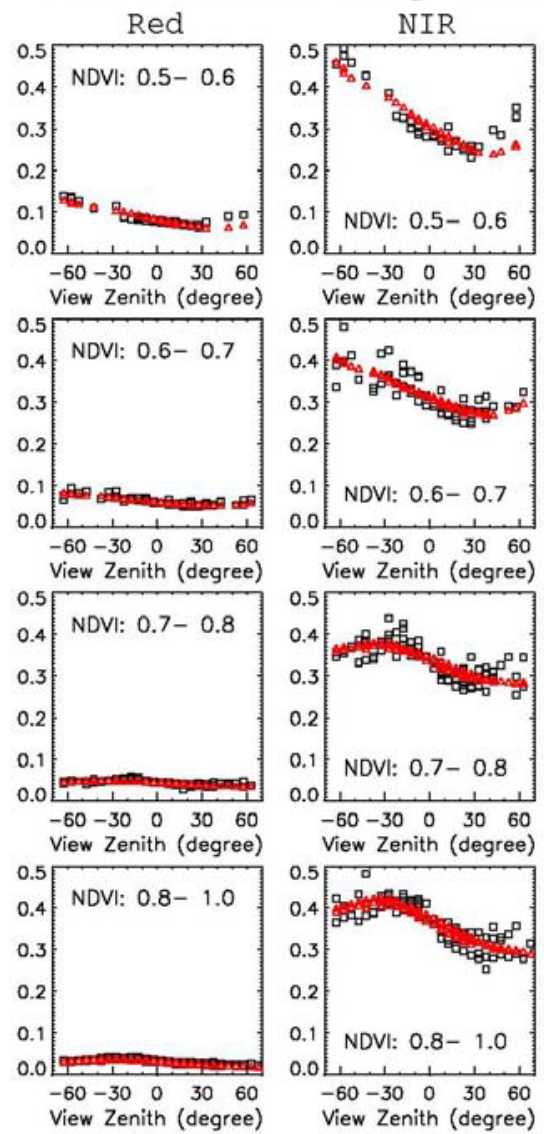


\section{Figure 7}

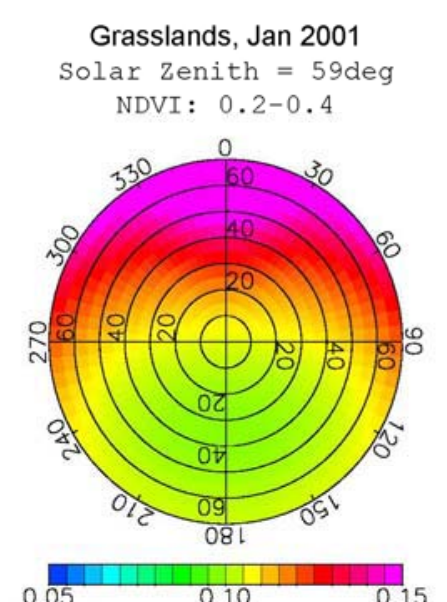

B1 (620-670 nm) Reflectance

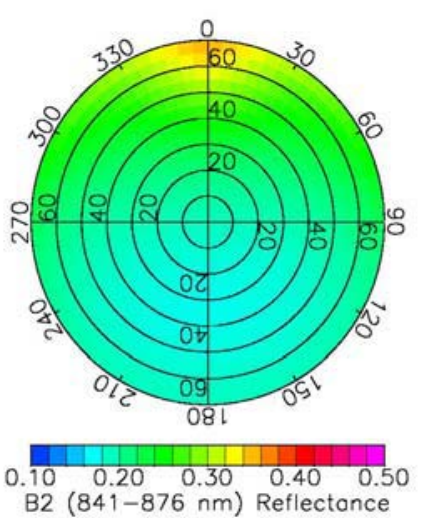

Grasslands, Apr 2001

Solar Zenith $=30 \mathrm{deg}$

NDVI: $0.3-0.4$

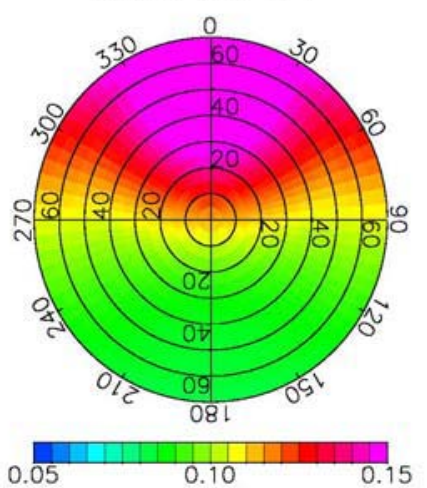

B1 $(620-670 \mathrm{~nm})$ Reflectonce

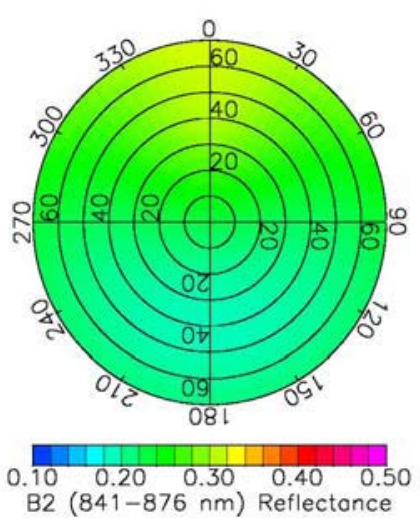

Grasslands, Jul 2001

Solar Zenith $=21 \mathrm{deg}$

NDVI: $0.6-0.8$

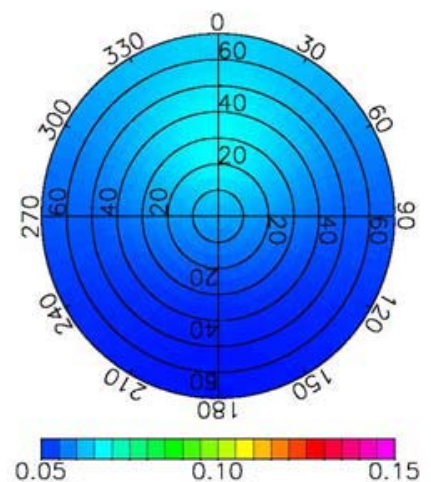

B1 $(620-670 \mathrm{~nm})$ Reflectance

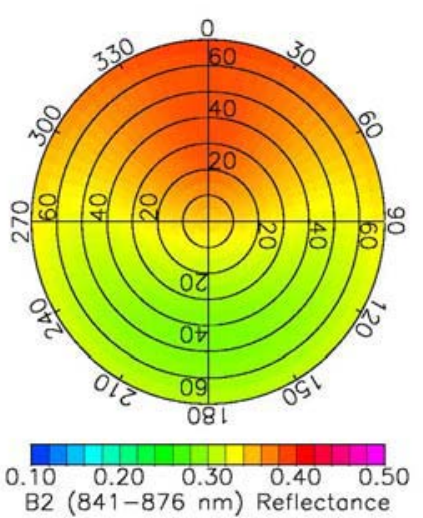

Grasslands, Oct 2001

Solar Zenith $=47 \mathrm{deg}$

NDVI : $0.4-0.6$

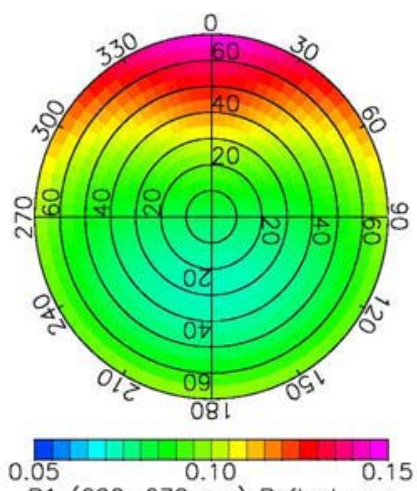

B1 $(620-670 \mathrm{~nm})$ Reflectance

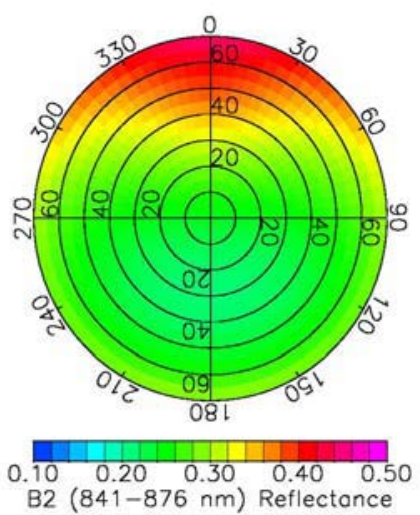


Figure 8

Pixel-based fitting
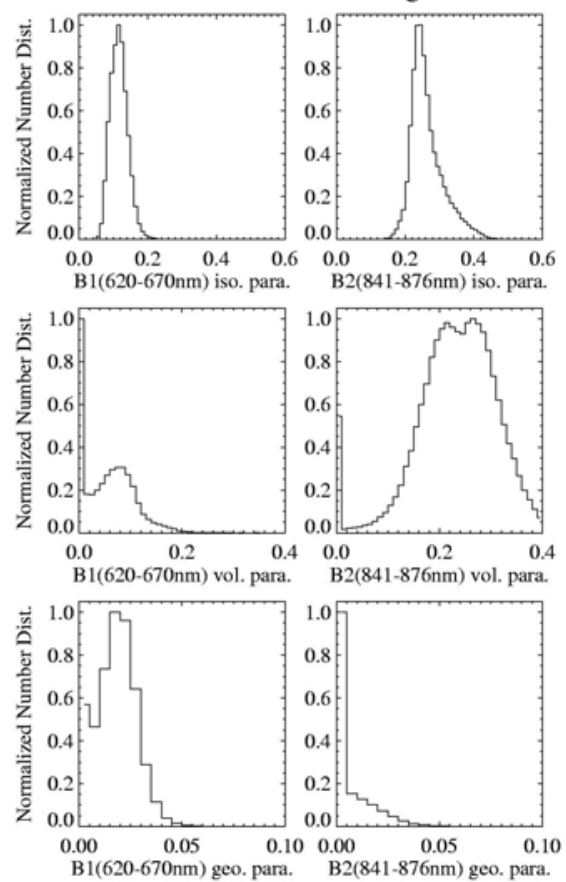

(a)
Landcover-based fitting
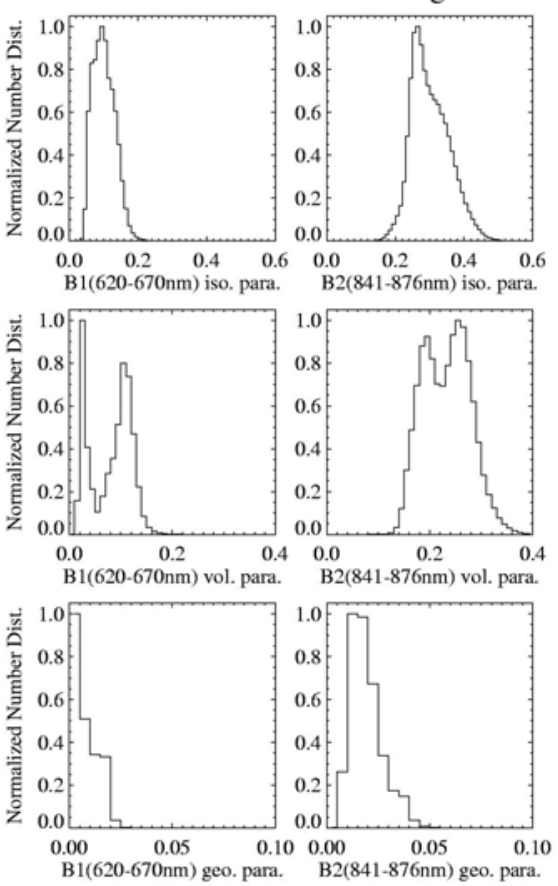

(b)

Deciduous Forest, April 2001

Pixel-based fitting
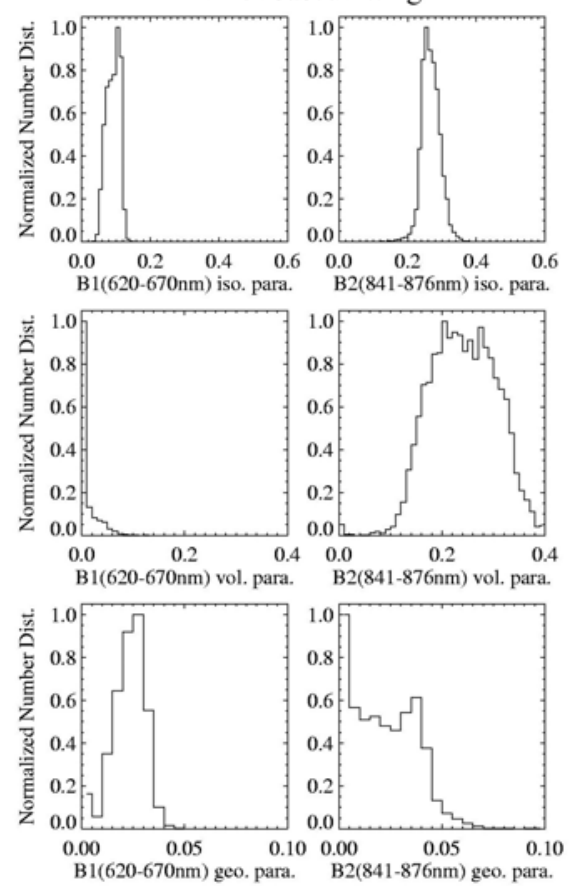

(c)
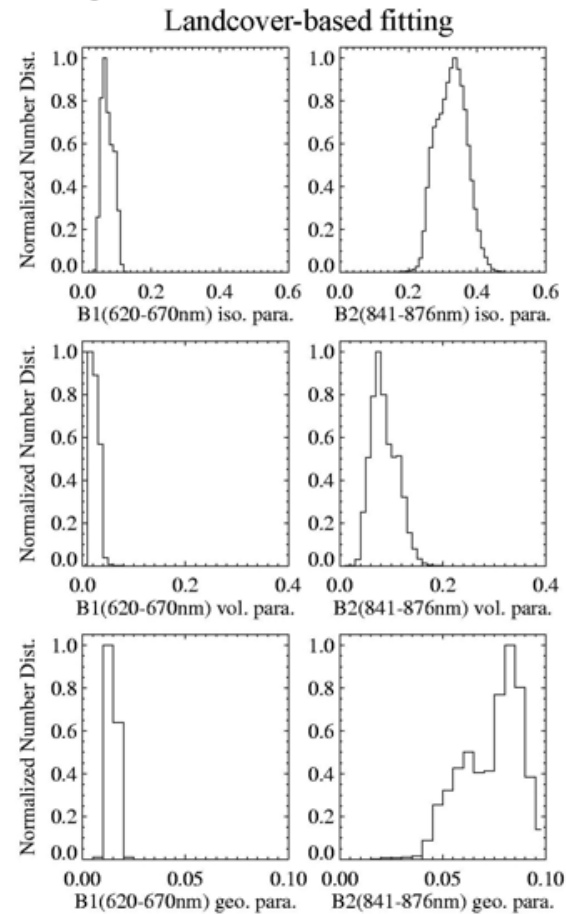

(d) 
Figure 9

MODIS LBF Albedo, April 2001

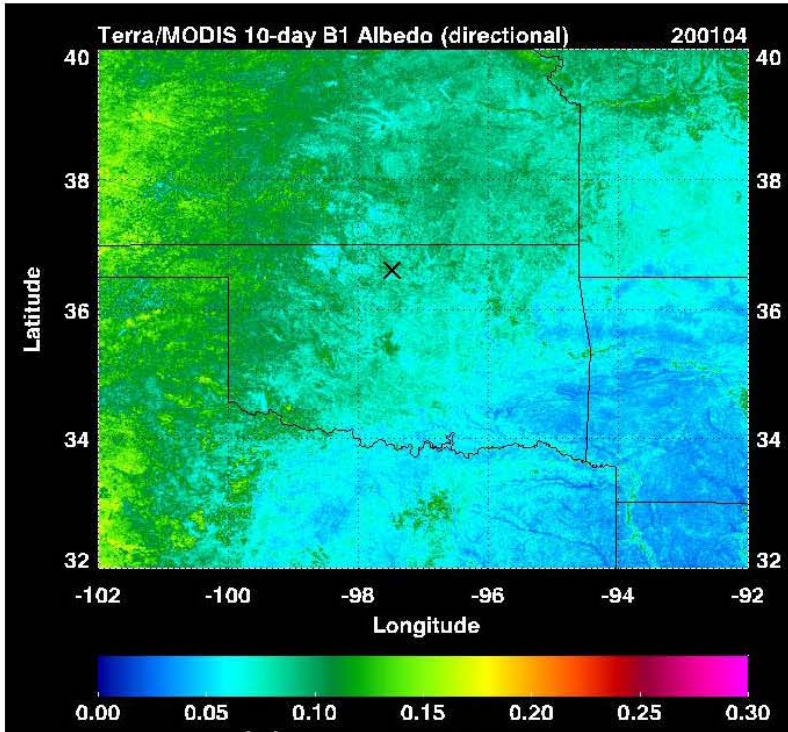

(a) B1 $(620-670 \mathrm{~nm})$ Albedo

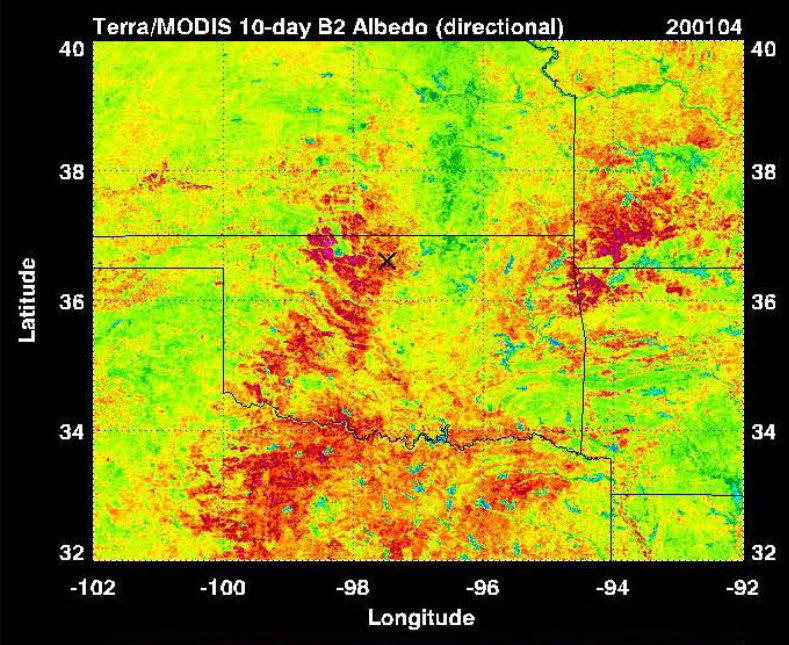

$\begin{array}{llllllllll}0.00 & 0.05 & 0.10 & 0.15 & 0.20 & 0.25 & 0.30 & 0.35 & 0.40 & 0.45\end{array}$ (b) B2 $(841-876 \mathrm{~nm})$ Albedo
MISR Albedo, April 2001
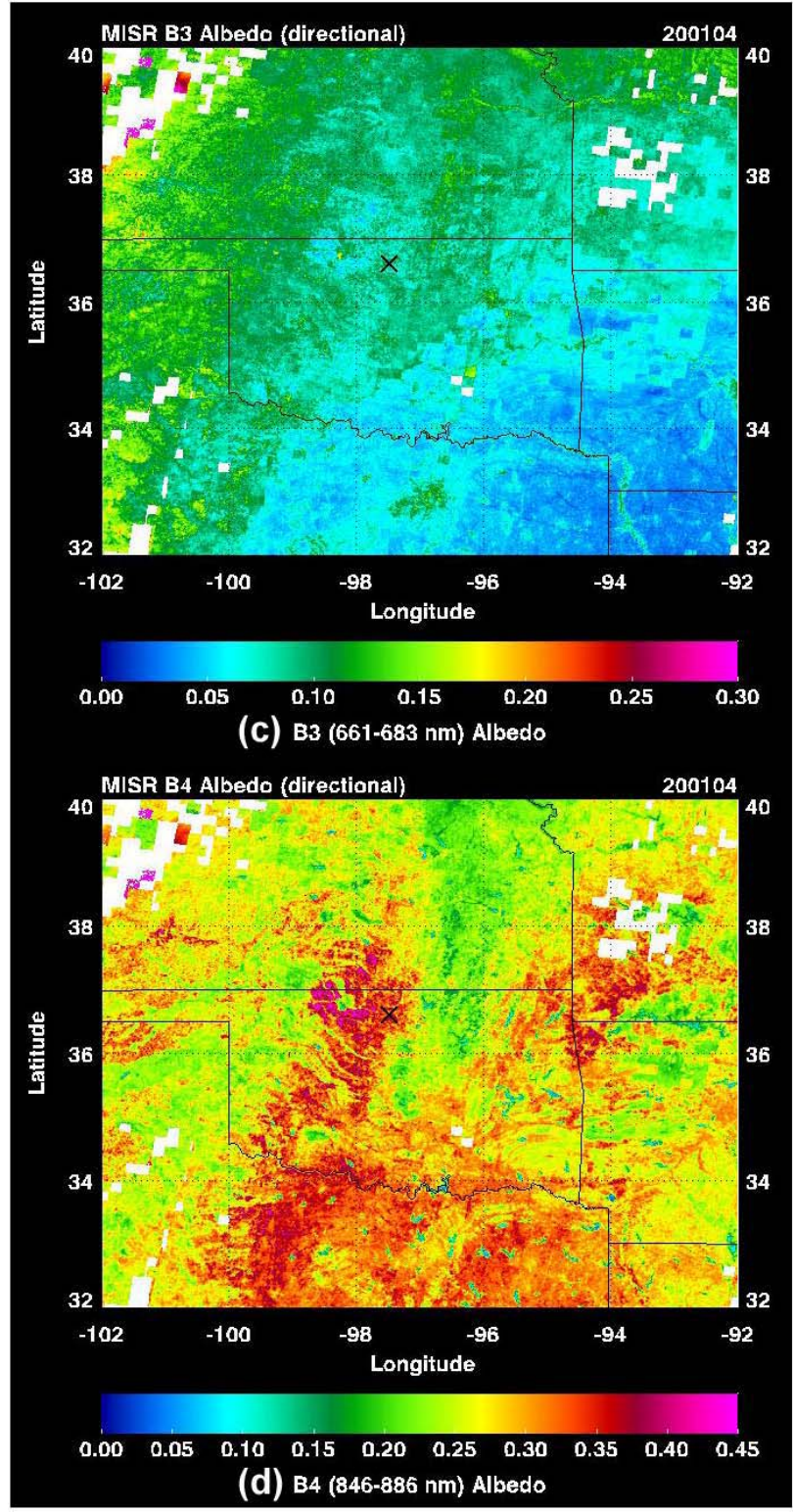
Figure 10
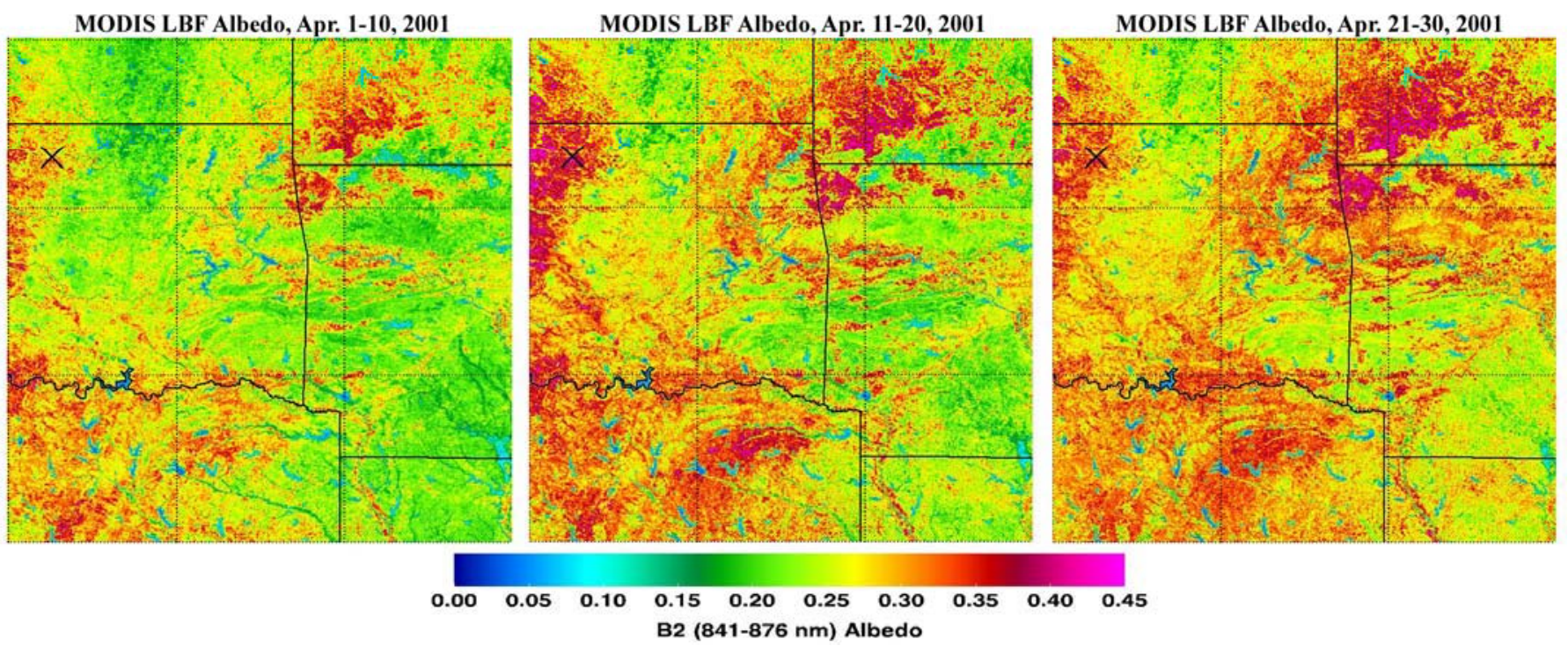


\section{Figure 11}
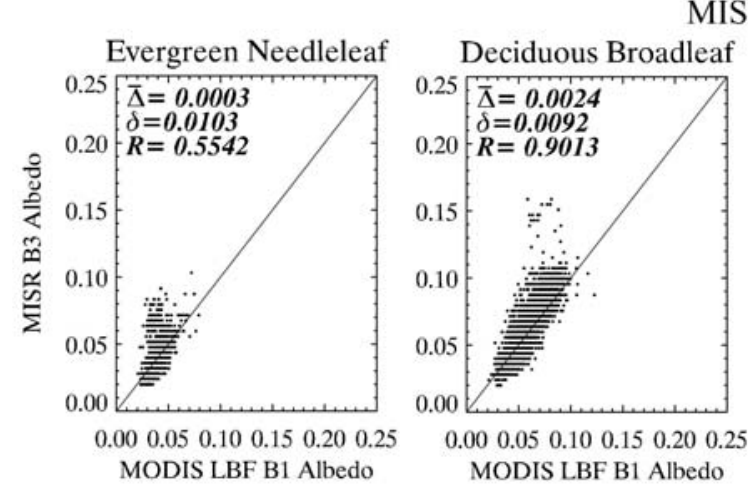

MISR vs MODIS Albedo, April 2001
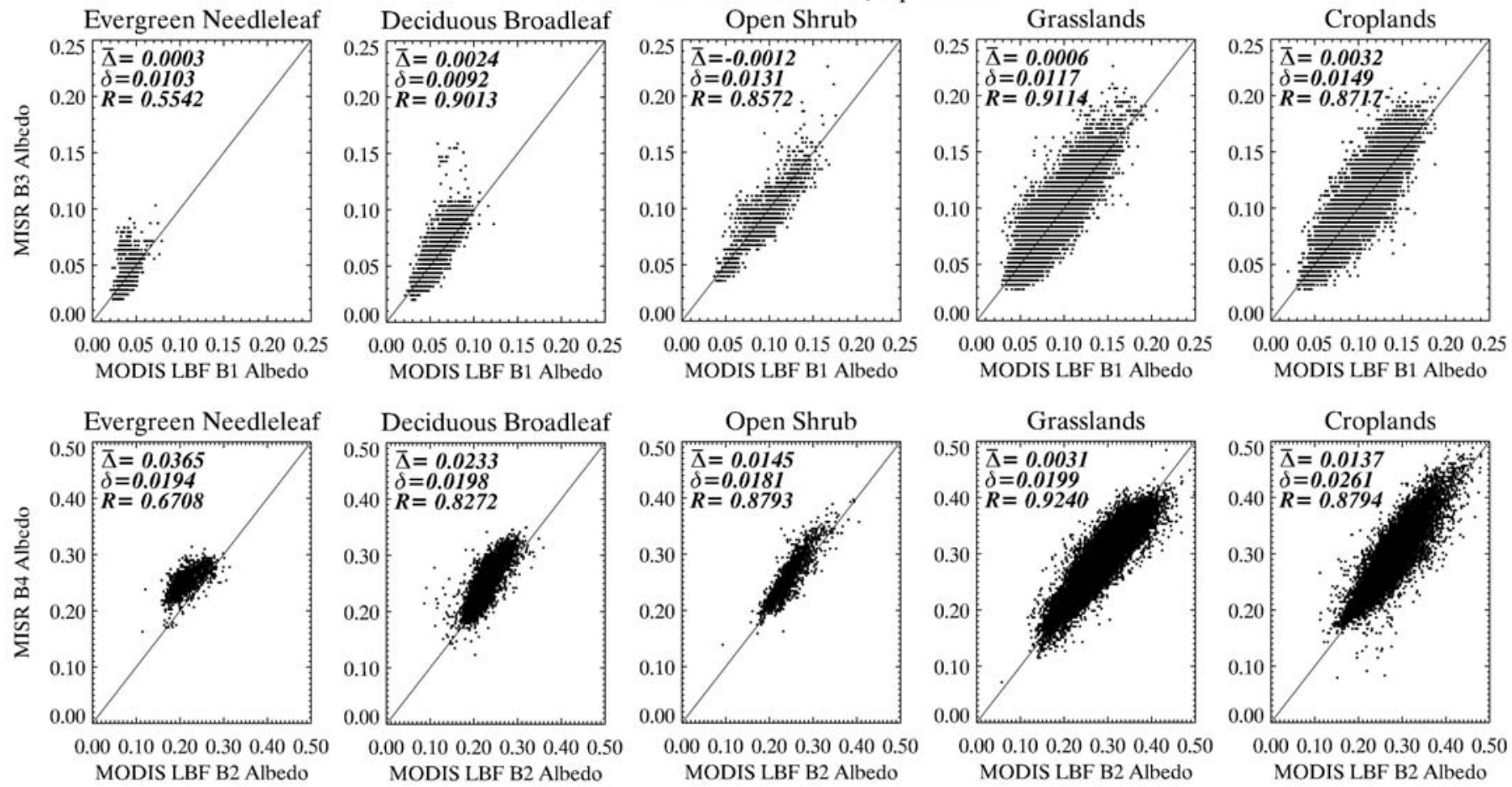
Figure 12

MISR vs MODIS Albedo, July 2001
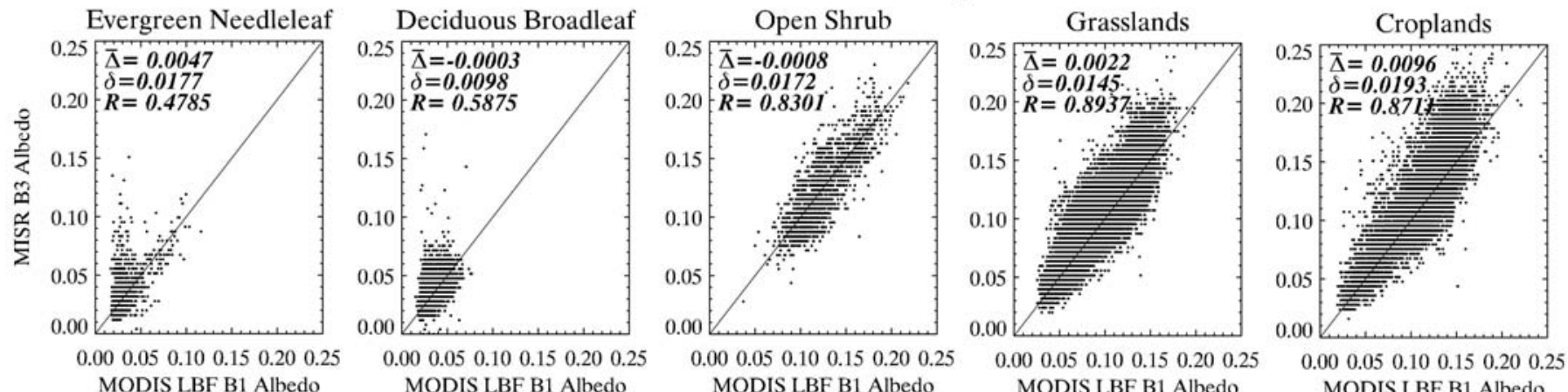

Evergreen Needleleaf
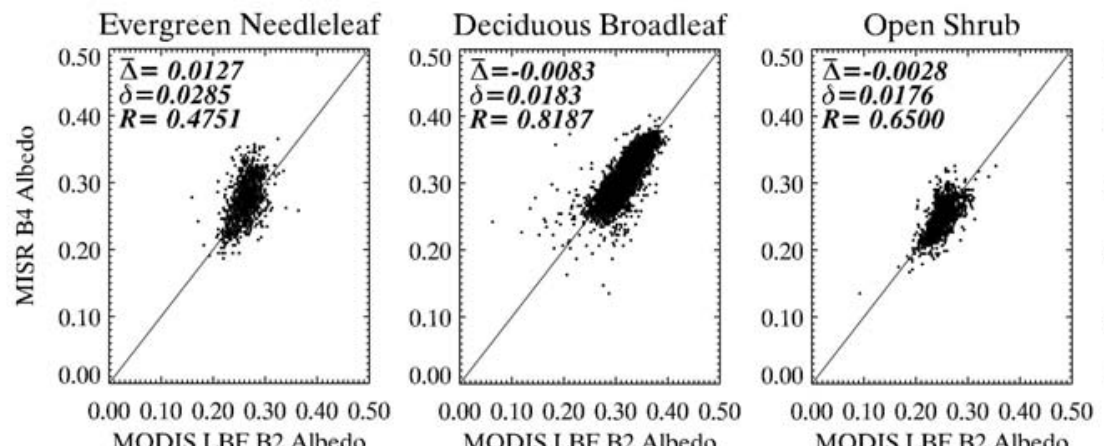

MODIS LBF B1 Albedo

MODIS LBF B1 Albedo
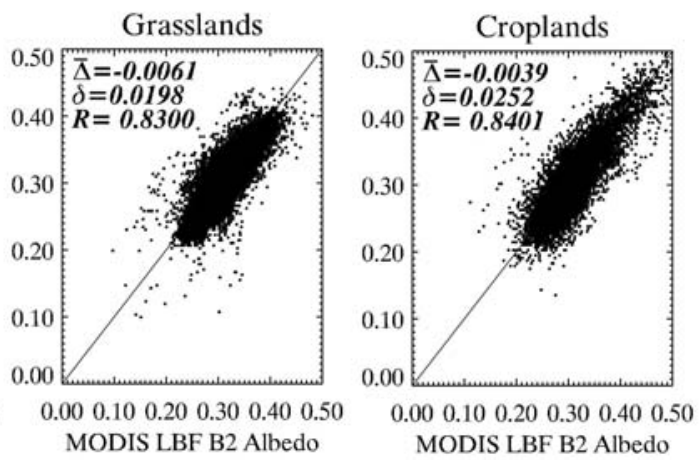


\title{
A method to derive the multi-spectral surface albedo consistent with MODIS from historical AVHRR and VGT satellite data
}

\author{
Alexander P. Trishchenko*, Yi Luo, Konstantin V. Khlopenkov and Shusen Wang \\ Canada Centre for Remote Sensing, Earth Sciences Sector, Natural Resources Canada, \\ 588 Booth Street, Ottawa, Ontario, Canada, K1A0Y7 \\ * Corresponding author: Tel 1-613-995 57 87; fax: 1-613-947 1385 \\ E-mail: trichtch@ccrs.nrcan.gc.ca
}

Submitted on 24 February, 2007 to Journal of Applied Meteorology and Climatology

Revised

July 16, 2007 
Abstract. Multi-spectral surface albedo and bi-directional properties are required for accurate determination of the surface and atmosphere solar radiation budget. A method is developed here to obtain time series of these surface characteristics consistent with MODIS using historical satellite observations with limited spectral coverage available from AVHRR/NOAA and VEGETATION/SPOT. Non-linear regression model was developed that relates retrievals from four spectral channels of VEGETATION/SPOT or three spectral channels of AVHRR/NOAA with retrieval from each of the seven MODIS channels designed for land applications. The model also takes into account the surface land cover type, normalized difference vegetation index and seasonal cycle. It was applied to generate surface albedo and bi-directional parameters of the seven MODIS like spectral channels at a 10-day interval for the 1995 to 2004 period over the US Southern Great Plains. The relative retrieval accuracy for MODIS channels replicated from AVHRR or VGT data was typically better than $5 \%$. Correlation coefficients between replicated and original data varied from 0.92 to 0.98 for all channels except MODIS channel 5, where it was lower $(0.77$ to 0.84$)$. The developed method provides valuable information for parameterization of spectral albedo in global climate models and can be extended to generate global multi-spectral data compatible with MODIS from historical AVHRR and VEGETATION/SPOT observations for the pre-MODIS era. 


\section{Introduction}

Surface albedo is a measure of the proportion of total incoming solar radiation reflected by the Earth's surface. It determines the amount of solar energy absorbed by the Earth's surface that drives surface hydrological and biochemical processes, influences productivity of the terrestrial and aquatic ecosystems, and affects the atmospheric circulation. Multi-spectral surface albedo and its variability are important for global climate model (GCM) development, atmospheric radiation applications, environmental monitoring and climate change studies (Charney et al., 1977; Dirmeyer and Shukla, 1994; Houghton et al., 2001, Wang et al., 2006).

While broadband shortwave (SW) albedo may serve as a good indicator of surface properties, an accurate modeling of the solar radiation budget components requires the multi-spectral information about the surface (Li et al, 2002; Halthore et al., 2005; Zhang et al., 2004). Uncertainties in specification of the surface albedo were identified as a possible source of discrepancy between the observed and modeled atmospheric absorption of solar radiation (Ackerman et al., 2003). There is a nonlinear relationship between the albedo and the surface/atmosphere absorbed radiation due to multiple scattering of photons in the atmosphere (Chandrasekhar, 1960; Liou, 2002). ). Due to anisotropy of the surface reflective properties, it is also necessary to account for dependence of the surface albedo on the solar zenith angle (Briegleb et al. 1986; Dickinson 1983). In general, these anisotropic properties are described by the bi-directional reflectance distribution function (BRDF) (Nicodemus, 1970).

The treatment of the surface albedo differs among various GCMs. Wang et al., (2006) analyzed 17 GCMs that participated in the International Panel on Climate Change (IPCC) Fourth Assessment Report (AR4) model intercomparison. Some of the models use one broad spectral band for surface albedo calculations, while others use two or more bands. Models developed at 
the Goddard Institute of Space Studies (GISS) use 6 spectral bands (Zhang et al., 2004). Many models separate the direct and diffuse albedo and include the solar zenith angle (SZA) dependence for specification of the direct albedo. The need for multi-spectral solar radiation transfer computations is now commonly recognized (Halthore et al., 2005; Morcrette, 2002). For example, the Community Climate System Model (CCSM) has 19 spectral intervals in the SW radiative transfer scheme (Collins et al., 2004). The solar radiative transfer schemes for the International Satellite Cloud Climatology Project (ISCCP) (Zhang et al., 2004) and the European Center for Medium Range Weather Forecast (ECMWF) [http://www.ecmwf.int/research/ifsdocs/CY28r1/index.html] have 6 spectral bands. Multiple spectral bands are also used in GCM radiation modeling of longwave (LW) radiation (Iacono et al., 2000). Thus, there is a pressing need for multi-spectral surface albedo data and its angular dependence within the climate and atmospheric radiation modeling community.

Figure 1 illustrates the importance of multi-spectral surface albedo information. It shows an example of the typical spectral albedo for several land classes, the spectral distribution of the downward solar flux for clear-sky and cloudy conditions and the location of spectral bands for several satellite sensors used in global mapping (Trishchenko et al., 2002a).. One can see substantial albedo variability among the atmospheric spectral windows. Except for the snowcovered and open soil surfaces, the albedo is typically very low $(<5 \%)$ in the UV region $(<0.4 \mu \mathrm{m})$, increases up to $15 \%$ in the photosynthetically active radiation (PAR) region $(0.4 \mu \mathrm{m}-$ $0.7 \mu \mathrm{m})$, and increases to as high as $40 \%-50 \%$ in the infrared spectral region $(0.7 \mu \mathrm{m}-1.2 \mu \mathrm{m})$. Beyond that interval, albedo decreases and can be as low as $5 \%$ to $10 \%$ in the shortwave infrared (SWIR) region $(2 \mu \mathrm{m}-2.5 \mu \mathrm{m})$. Snow/ice, barren land and desert surfaces exhibit different spectral properties. Snow, for example, has a very high reflectance, frequently up to $80 \%$ or even 
higher in the spectral range $\lambda<1 \mu m$, and a very low reflectance of a few percent in the SWIR range $\lambda>1.5 \mu \mathrm{m}$. It is clear from Figure 1 that accurate determination of the surface and atmosphere radiation budget requires precise knowledge of the surface albedo within the major atmospheric windows. Having one or two broadband values for the entire SW may occasionally provide good results, but this simplification may lead to substantial biases in many important cases.

The spectral dependence of the surface albedo leads to a dependence of broadband albedo on the atmospheric conditions that alter the spectral distribution of atmospheric downward irradiance (Lyapustin, 1999). In such a case, spectral albedos contribute to broadband albedo with different weights proportional to the amount of radiative energy reaching the surface within the spectral bands. This is illustrated in Figure 2, which shows examples of SW broadband surface albedo dependence on sky-conditions for ocean, grassland, and evergreen forest surface types. The spectral and angular surface albedo distributions were taken from Rutan and Charlock (1997) and $\mathrm{Li}$ et al. (2002). The computations were conducted using the adding-doubling radiative transfer model described by Halthore et al., (2005) as model \#2, and applied for the standard clear-sky mid-latitude summer conditions and $S c$ clouds of optical thickness 5 (thin) and 100 (thick) at $0.55 \mu \mathrm{m}$ (Stephens, 1978). Dependence of the surface albedo on the solar zenith angle (SZA) $\theta_{0}$ under clear-sky conditions results from the directional properties of the surface reflectance and effect of the atmosphere. Under overcast conditions the surface albedo does not depend on the solar zenith angle as the downward radiation consists of a diffuse field with no directional behavior. Albedo of vegetated surfaces, however, depends on the cloud optical depth. This is due to the difference between visible, near-infrared (NIR) and SWIR 
spectral albedo and the fact that the ratio of spectral downward fluxes between different spectral bands depends on the cloud optical depth.

Satellite remote sensing is the most practical way to estimate the surface albedo over large regions or globally. Coarse resolution radiation budget sensors like the Earth Radiation Budget Experiment (ERBE), the Scanner for Radiation Budget (ScaRaB), and the Clouds and the Earth's Radiant Energy System (CERES) are used to map the broadband SW albedo distribution (Barkstrom et al., 1989; Kandel et al, 1998; Wielicki et al., 1996). There are three popular types of satellite sensors employed for large scale spectral surface albedo mapping at moderate $(\sim 1 \mathrm{~km})$ spatial resolution (Barnsley et al., 2000, Csiszar and Gutman, 1999; Disney et al., 2004; Schaaf et al., 2002; Luo et al., 2005; Strugnell et al., 2001; Trishchenko et al., 2007). They are the Advanced Very High Resolution Radiometer (AVHRR) onboard the NOAA platforms, the VEGETATION (VGT) sensor onboard of the SPOT spacecrafts, and the Moderate-resolution Imaging Spectroradiometer (MODIS) onboard the Terra and Aqua spacecrafts. The longest time series of observations are available from the AVHRR/ NOAA sensors. They began with AVHRR/TIROS-N and AVHRR/NOAA-6 in the late 1970's and early 1980's (Kidwell, 1998; Goodrum et al., 2000). The latest AVHRR instrument on NOAA platform was launched in May, 2005 onboard NOAA-18. The new AVHRR instrument was launched onboard the European MetOp platform in November, 2006. Depending on the sensor, the AVHRR/1 and AVHRR/2 instruments have 5 or 6 spectral channels with bands located in the visible (B1), NIR (B2), and 3.7 $\mu \mathrm{m}$ spectral region $(B 3 B)$, and two bands in thermal regions $10-12 \mu \mathrm{m}$ (B4-B5) (except for AVHRR/ 1 where band 5 was a duplicate of band4). The latest instruments (AVHRR/3) have 6 spectral channels with the addition of a spectral band in the $1.6 \mu \mathrm{m}$ spectral region $(B 3 A)$, which works interchangeably with channel $3.7 \mu \mathrm{m}$. The first VEGETATION (VGT) sensor onboard 
SPOT-4 platform developed by the French Space Agency CNES became operational in 1998. There are currently two VGT sensors providing global surface products at 1-km resolution in the blue $(B 0)$, red $(B 1)$, NIR $(B 3)$, and SWIR 1.6 $\mu \mathrm{m}(B 4)$ spectral regions. Note that there is some mismatch in the identification of channels between various sensors. For consistency, we preserve the identification proposed by data providers and instrument developers.

A new opportunity for surface spectral albedo and BRDF mapping has emerged since 2000 with the launch of MODIS (Salomonson et al., 1989; Schaaf et al., 2002). The MODIS sensor with 36 spectral bands provides much improved capacity for characterization of surface spectral albedo (Schaaf et al., 2002; Luo et al., 2005). With more spectral bands, the MODIS data can also be utilized to retrieve the broadband albedo (Liang, 2003). MODIS data are calibrated to an absolute scale using an onboard calibration technique (Guenther et al., 1998; Xiong et al., 2002; Xiong and Barnes, 2006), whereas sensors like AVHRR/NOAA have no in-flight radiometric characterization for channels $B 1, B 2$ and $B 3 A$ and rely on post-launch calibration processes (Cao et al., 2004; Rao and Chen, 1996). Although less sophisticated, the VGT data processing system also provides surface reflectance and BRDF parameters that were successfully compared to MODIS results (Disney et al., 2004; Duchemin et al., 2002; Duchemin and Maisongrande, 2002; Hagolle et al., 2004; Samain et al., 2005, 2006).

Though a great source of information, MODIS data are only available since February, 2000. Long term and consistent records of surface spectral albedo are required for studies related to surface property analysis and for historical GCM model runs and validation. In this study, we propose to reconstruct the historical surface spectral albedo and BRDF consistent with MODIS data by using AVHRR and VGT observations. The method has been implemented over the US Southern Great Plains (SGP) region in the framework of the project supported by the US 
Department of Energy Atmospheric Radiation Measurement (ARM) Program (Ackerman and Stokes, 2003). The selected study region is $8^{\circ} \times 10^{\circ}$ with upper left corner $\left(40^{\circ} \mathrm{N}, 102^{\circ} \mathrm{W}\right)$ and lower right corner $\left(32^{\circ} \mathrm{N} ; 92^{\circ} \mathrm{W}\right)$.

The basic premise of the reconstruction approach is that the shape of the surface albedo spectrum and the bi-directional properties are mainly determined by the land cover type, its state (biomass amount, water stress), and seasonal factors that affect the soil moisture, solar illumination conditions and canopy/surface structure (Bowker et al., 1985; Luo et al, 2005). In other words, within the same land cover type in similar conditions there exists a certain correlation among the albedo values observed in different spectral bands. If this correlation is robust, then it can be used to predict the spectral albedo from AVHRR and VGT data. Because there is an overlap in operation between all the moderate resolution sensors AVHRR, VGT and MODIS, this hypothesis can be tested on the real data.

For consistency with VGT and AVHRR processing, we developed an independent MODIS data processing system that delivers 10-day albedo/BRDF products as described by Luo et al. (2005). This system is different from the standard MODIS data processing system that generates 16-day products (Schaaf et al., 2002). When aggregated over compatible temporal and spatial scales, our MODIS results are consistent with standard MODIS archival products as demonstrated by Luo et al. (2005).

\section{Albedo and BRDF: definition and nonlinear impact on radiation budget}

Albedo is an integrated product of the downward radiance field $L_{\lambda}^{i}\left(t, \theta_{i}, \phi_{i}\right)$ and the BRDF function $f_{\lambda}\left(t, \theta_{r}, \phi_{r}, \theta_{i}, \phi_{i}\right)$, where $\lambda$ denotes a wavelength, $t$ is the time to account for temporal changes in surface properties and radiance field. The BRDF determines the reflective properties of an image pixel for the radiative energy at wavelength $\lambda$ incident upon the surface 
from direction $\left(\theta_{i}, \phi_{i}\right)$ and reflected at angle $\left(\theta_{r}, \phi_{r}\right)$ with relative azimuth $\Delta \phi=\phi_{r}-\phi_{i}$ between the direction of incidence and reflection (Nicodemus, 1970). To simplify, we will omit the time dependence.

In general, it is assumed that the BRDF depends only on the relative azimuth angle, i.e.

$$
f_{\lambda}\left(\theta_{r}, \phi_{r}, \theta_{i}, \phi_{i}\right)=\frac{d L_{\lambda}^{r}\left(\theta_{r}, \theta_{i}, \Delta \phi\right)}{L_{\lambda}^{i}\left(\theta_{i}, \phi_{i}\right) \cos \theta_{i} d \omega_{i}}=f_{\lambda}\left(\theta_{r}, \theta_{i}, \Delta \phi\right)
$$

The spectral monochromatic albedo $\alpha_{\lambda}$ (also called bihemispherical reflectance, Nicodemus, (1970)) is the ratio of the surface radiant exitance $M_{\lambda}\left(\theta_{0}\right)$ over the surface downwelling irradiance $E_{\lambda}\left(\theta_{0}\right)$, and depends on the solar zenith angle $\theta_{0}$ :

$$
\alpha_{\lambda}\left(\theta_{0}\right)=\frac{M_{\lambda}\left(\theta_{0}\right)}{E_{\lambda}\left(\theta_{0}\right)},
$$

where

$$
\begin{aligned}
& M_{\lambda}\left(\theta_{0}\right)=\int_{2 \pi} L_{\lambda}^{r}\left(\theta_{r}, \phi_{r}\right) \mu_{r} d \omega_{r}=\iint_{2 \pi, 2 \pi} f_{\lambda}\left(\theta_{r}, \theta_{i}, \Delta \phi\right) L_{\lambda}^{i}\left(\theta_{0}, \theta_{i}, \phi_{i}\right) \mu_{i} d \omega_{i} \mu_{r} d \omega_{r} \\
& E_{\lambda}\left(\theta_{0}\right)=\int_{2 \pi} L_{\lambda}^{i}\left(\theta_{0}, \theta_{i}, \phi_{i}\right) \mu_{i} d \omega_{i}
\end{aligned}
$$

The notation $L_{\lambda}^{i}\left(\theta_{0}, \theta_{i}, \phi_{i}\right)$ with explicit inclusion of SZA $\theta_{0}$ is used in Equations (2a-b) above to emphasize impact of the atmospheric scattering on the surface downward radiance field. For non-scattering atmosphere (black-sky case) that approximately corresponds to clear-sky conditions, the downward solar radiance field can be considered as a collimated beam, i.e. $L_{\lambda}^{i}\left(\theta_{0}, \theta_{i}, \phi_{i}\right)=E_{\lambda}^{0} \delta\left(\mu_{i}-\mu_{0}\right) \delta\left(\phi_{i}-\phi_{0}\right)$. In such a case, albedo is called the direct albedo and can be expressed as 


$$
\alpha_{\lambda}\left(\theta_{0}\right)=\int_{2 \pi} f_{\lambda}\left(\theta_{r}, \theta_{0}, \phi_{r}\right) \mu_{r} d \omega_{r}
$$

where $\mu_{(i, r, 0)}=\cos \theta_{(i, r, 0)}$, and $d \omega_{(r, i)}=d \cos \theta_{(r, i)} d \phi_{(r, i)}$

Under overcast conditions the surface downward solar flux is approximated by an isotropic field, $L_{\lambda}^{i}\left(\theta_{0}, \theta_{i}, \Delta \phi\right)=L_{\lambda}^{0}$. Then, albedo is called the diffuse albedo and can be expressed as

$$
\bar{\alpha}_{\lambda}=2 \int_{0}^{1} \mu d \mu \alpha_{\lambda}(\theta) .
$$

Usually, the BRDF is parameterized as a combination of kernel functions (Strahler et al., 1999)

$$
f_{\lambda}\left(\theta_{r}, \theta_{0}, \phi\right)=\sum_{k} f_{k}(\lambda) F_{k}\left(\theta_{r}, \theta_{0}, \phi\right)
$$

Then, for the monochromatic case, one can derive the following expressions for the direct and diffuse albedo

$$
\begin{aligned}
& \alpha_{\lambda}\left(\theta_{0}\right)=\sum_{k} f_{k}(\lambda) h_{k}\left(\theta_{0}\right) \\
& \bar{\alpha}_{\lambda}=\sum_{k} f_{k}(\lambda) \bar{h}_{k}
\end{aligned}
$$

where

$$
\begin{aligned}
& h_{k}\left(\theta_{0}\right)=\int_{2 \pi} F_{k}\left(\theta, \theta_{0}, \phi\right) \mu d \mu d \phi \\
& \bar{h}_{k}=\int_{0}^{1} h_{k}(\theta) \mu d \mu
\end{aligned}
$$

A convenient choice of kernel functions widely used for BRDF parameterization is the RossThick-Li-Sparse (RTLS) parameterization employed in MODIS data processing (Strahler et al., 1999; Wanner et al., 1995). This model is reciprocal (i.e. symmetrical relative to incoming and outgoing directions) and contains three kernels

$$
f_{\lambda}\left(\theta_{r}, \theta_{i}, \phi\right)=f_{0}(\lambda)+f_{1}(\lambda) F_{1}\left(\theta_{r}, \theta_{i}, \phi\right)+f_{2}(\lambda) F_{2}\left(\theta_{r}, \theta_{i}, \phi\right)
$$


where function $F_{1}$ is the Ross-Thick kernel representing volumetric scattering from a dense leaf canopy based on a single-scattering approximation of radiative transfer theory (Ross, 1981; Roujean et al., 1992), function $F_{2}$ is the Li-Sparse kernel derived from the geometric-optical mutual shadowing model by assuming a sparse ensemble of surface objects (Li and Strahler, 1992). Parameters $f_{0}(\lambda), f_{1}(\lambda)$ and $f_{2}(\lambda)$ are the amplitudes related to the isotropic, volumetric and geometric components, respectively. Expressions for these kernel functions can be found in Strahler et al., (1999).

For the case of finite spectral intervals, the direct and diffuse surface albedo are given by

$$
\begin{gathered}
\alpha_{\Delta \lambda}\left(\theta_{0}\right)=\sum_{k} h_{k}\left(\theta_{0}\right) w_{k}\left(\theta_{0}\right) \\
\bar{\alpha}_{\Delta \lambda}=\sum_{k} \bar{h}_{k} \bar{w}_{k},
\end{gathered}
$$

where

$$
w_{k}\left(\theta_{0}\right)=\frac{\int_{\lambda_{1}}^{\lambda_{2}} d \lambda f_{k}(\lambda) E_{\lambda}^{0}\left(\theta_{0}\right)}{\int_{\lambda_{1}}^{\lambda_{2}} d \lambda E_{\lambda}^{0}\left(\theta_{0}\right)}, \quad \bar{w}_{k}=2 \frac{\int_{\lambda_{1}}^{\lambda_{2}} d \lambda f_{k}(\lambda) L_{\lambda}^{0}}{\int_{\lambda_{1}}^{\lambda_{2}} d \lambda L_{\lambda}^{0}}
$$

Equations (10)-(12) emphasize the dependence of broadband albedo on the spectral distribution of direct $E_{\lambda}^{0}\left(\theta_{0}\right)$ and diffuse $L_{\lambda}^{0}$ downward fields.

The impact of the surface albedo on the surface downward flux, surface absorbed flux and atmospheric absorption is nonlinear. According to Chandrasekhar (1960) and Liou (2002) the surface irradiance $E_{\lambda}\left(\theta_{0}\right)$ and TOA radiant exitance $M_{\lambda}^{T O A}\left(\theta_{0}\right)$ can be expressed as 


$$
\begin{aligned}
& M_{\lambda}^{T O A}\left(\theta_{0}\right)=M_{\lambda, 0}^{T O A}\left(\theta_{0}\right)+\frac{\alpha_{\lambda}}{1-\alpha_{\lambda} \bar{S}_{\lambda}\left(\theta_{0}\right)} E_{\lambda, 0}\left(\theta_{0}\right) \bar{T}_{\lambda}\left(\theta_{0}\right) \\
& E_{\lambda}\left(\theta_{0}\right)=E_{\lambda, 0}\left(\theta_{0}\right)+\frac{\alpha_{\lambda}}{1-\alpha_{\lambda} \bar{S}_{\lambda}\left(\theta_{0}\right)} E_{\lambda, 0}\left(\theta_{0}\right) \bar{S}_{\lambda}\left(\theta_{0}\right)
\end{aligned}
$$

where $E_{\lambda, 0}\left(\theta_{0}\right)$ and $M_{\lambda, 0}^{T O A}\left(\theta_{0}\right)$ are surface irradiance and TOA radiant exitance for the standard case of non-reflecting (black) surface, $\bar{S}_{\lambda}\left(\theta_{0}\right)$ is the atmosphere spherical albedo (atmospheric backscattering), and $\bar{T}_{\lambda}\left(\theta_{0}\right)$ is the total atmospheric spherical transmittance. For simplicity, Equation (13) is written for the case of a Lambertian surface (Equations 6.3.69-6.3.72 of Liou (2002)). The expression for the surface absorbed flux is

$$
\Delta E_{\lambda}\left(\theta_{0}\right)=\left(1-\alpha_{\lambda}\right) E_{\lambda}\left(\theta_{0}\right)=\frac{1-\alpha_{\lambda}}{1-\alpha_{\lambda} \bar{S}_{\lambda}\left(\theta_{0}\right)} E_{\lambda, 0}\left(\theta_{0}\right)
$$

The expression for the atmosphere absorbed flux is

$$
A_{\lambda}\left(\theta_{0}\right)=\text { net TOA }- \text { net Surface }=\left[S_{0, \lambda} \mu_{0}-M_{\lambda}^{T O A}\left(\theta_{0}\right)\right]-\left(1-\alpha_{\lambda}\right) E_{\lambda}\left(\theta_{0}\right) \text {, }
$$

which is reduced to

$$
A_{\lambda}\left(\theta_{0}\right)=\Delta A_{\lambda, 0}\left(\theta_{0}\right)+\alpha_{\lambda} E_{\lambda, 0}\left(\theta_{0}\right) \frac{1-\bar{S}_{\lambda}\left(\theta_{0}\right)-\bar{T}_{\lambda}\left(\theta_{0}\right)}{1-\alpha_{\lambda} \bar{S}_{\lambda}\left(\theta_{0}\right)}=\Delta A_{\lambda, 0}\left(\theta_{0}\right)+E_{\lambda, 0}\left(\theta_{0}\right) \frac{\alpha_{\lambda} \bar{A}_{\lambda}\left(\theta_{0}\right)}{1-\alpha_{\lambda} \bar{S}_{\lambda}\left(\theta_{0}\right)}
$$

where $\Delta A_{\lambda, 0}\left(\theta_{0}\right)$ is the atmosphere absorption for the standard case of a black surface $(\alpha=0)$, $S_{0, \lambda}$ is the solar extraterrestrial irradiance at wavelength $\lambda, \bar{A}_{\lambda}\left(\theta_{0}\right)=1-\bar{S}_{\lambda}\left(\theta_{0}\right)-\bar{T}_{\lambda}\left(\theta_{0}\right)$ is the average spherical atmospheric absorption for the standard case of a black surface.

$$
\Delta A_{\lambda, 0}\left(\theta_{0}\right)=S_{0, \lambda} \mu_{0}-M_{\lambda, 0}^{T O A}\left(\theta_{0}\right)-E_{\lambda, 0}\left(\theta_{0}\right)
$$

Equation (15) indicates that an additional atmospheric absorption occurs due to the surface reflection. This additional absorption is determined by the second term in Eq. (15), which has a simple physical interpretation. It is an additional absorption of the solar flux that is reflected 
from the surface into the atmosphere $E_{\lambda, 0}\left(\theta_{0}\right) \frac{\alpha_{\lambda}}{1-\alpha_{\lambda} \bar{S}_{\lambda}\left(\theta_{0}\right)}$. The quantity $\bar{A}_{\lambda}\left(\theta_{0}\right)$ determines the rate of absorption of this additional flux, which has a meaning of the atmospheric diffusive absorption.

The nonlinear term in Equations (13)-(15) has an important implication for computing the band average albedo $\alpha_{\Delta \lambda}$ defined as the ratio of upward spectrally integrated irradiance to downward spectrally integrated irradiance

$$
\alpha_{\Delta \lambda}=\left[\int_{\lambda_{1}}^{\lambda_{2}=\lambda_{1}+\Delta \lambda} d \lambda \frac{\alpha_{\lambda} E_{\lambda, 0}\left(\theta_{0}\right)}{1-\alpha_{\lambda} \bar{S}_{\lambda}\left(\theta_{0}\right)}\right] /\left[\int_{\lambda_{1}}^{\lambda_{2}=\lambda_{1}+\Delta \lambda} d \lambda \frac{E_{\lambda, 0}\left(\theta_{0}\right)}{1-\alpha_{\lambda} \bar{S}_{\lambda}\left(\theta_{0}\right)}\right]
$$

Even for the case of wavelength independent atmospheric properties, i.e. $E_{\lambda, 0}\left(\theta_{0}\right)=E_{0}\left(\theta_{0}\right)$ and $\bar{S}_{\lambda}\left(\theta_{0}\right)=\bar{S}\left(\theta_{0}\right)$, the band average surface albedo in the first order of magnitude for $\bar{S}\left(\theta_{0}\right)$ is defined as

$$
\alpha_{\Delta \lambda}=\bar{\alpha}+\bar{S}\left(\theta_{0}\right)\left(\bar{\alpha}^{2}-\bar{\alpha}^{2}\right)=\bar{\alpha}+\bar{S}\left(\theta_{0}\right) \sigma_{\Delta \lambda}^{2}(\alpha)
$$

where $\bar{\alpha}=\frac{1}{\Delta \lambda} \int_{\lambda_{1}}^{\lambda_{2}=\lambda_{1}+\Delta \lambda} \alpha_{\lambda} d \lambda, \overline{\alpha^{2}}=\frac{1}{\Delta \lambda} \int_{\lambda_{1}}^{\lambda_{2}=\lambda_{1}+\Delta \lambda} \alpha_{\lambda}^{2} d \lambda$ and $\sigma_{\Delta \lambda}^{2}(\alpha)$ denotes the spectral dispersion of albedo over the spectral band $\left[\lambda_{1}+\Delta \lambda ; \lambda_{1}\right]$. Therefore, if the spectral albedo is not a wavelength independent quantity, then the average albedo is not equal to the mathematical average, but depends on the spectral dispersion of albedo within spectral band $\Delta \lambda$ and the atmospheric backscattering $\bar{S}\left(\theta_{0}\right)$.

\section{Surface properties over the SGP area}

The surface reflective properties are determined by vegetation and soil properties (land cover type) and variable seasonal factors, such as presence or absence of snow, seasonal cycle of 
vegetation, soil moisture, farming activities, and the degree of water and nutrient stress on vegetation. The land cover type distribution over the study area was described by Trishchenko et al. (2004). The area is mostly dominated by cropland and pasture with rather intensive agricultural activities throughout the year. The southeast part of the SGP region contains various forest types: deciduous, evergreen and mixed.

To study the detailed surface properties in the vicinity of the ARM Central Facility (CF) (36 $37^{\prime} \mathrm{N} ; 97^{\circ} 30^{\prime} \mathrm{W}$ ) representative of cropland and pasture types, we have conducted several field campaigns referred to as Intensive Operational Periods (IOPs) in February, May, September, and October from 2002 to 2005 . These periods represent several typical phases of growing season for this agricultural region. A detailed land cover map of this region for each IOP was generated from the high-resolution satellite imagery from Landsat-7 ETM+ and our ground survey data. Figure 3 shows two Landsat images and corresponding land cover maps for IOPs conducted in late summer 2002 and May 2003. Figures 3a-d show the same area of about $10 \times 10 \mathrm{~km}^{2}$, with the CF site at the center. A strip of thin $\mathrm{Ci}$ cloud is visible in Figure 3b. This, however, does not strongly impact the detection of seasonal differences in the state of vegetation and distribution of the spectral albedo. In springtime (Figure $3 \mathrm{~b}$ and $\mathrm{d}$ ), the wheat fields are in a well developed stage and dominate the area. The vegetation over the pasture and grassland is less developed. However, in late-summer, after harvest, (Figure $3 \mathrm{a}$ and $\mathrm{c}$ ) the bare soil fields dominate. Therefore, the seasonal dynamics of natural and managed lands are important in describing the surface reflectance properties.

The land cover map used in this paper for the entire area of $8^{\circ} \times 10^{\circ}$ was derived from the National Land Cover Dataset (NLCD) produced by the U.S. Geological Survey (USGS) (http://landcover.usgs.gov/natllandcover.asp). The NLCD was compiled from Landsat Thematic 
Mapper (TM) satellite multi-band imagery and supplemented by various ancillary parameters. The original NLCD land cover dataset (21 thematic classes at 30-meter spatial resolution) was resampled and aggregated to match the spatial resolution of the MODIS $(0.5 \mathrm{~km})$ and VGT or AVHRR $(1 \mathrm{~km})$ imagery. Each land cover class in the resampled dataset was then converted to the International Geosphere-Biosphere Programme (IGBP) equivalent class as described by Latifovic et al. (2004). Finally, the IGBP classification was employed because of its frequent application in climate modeling and ecological studies. Based on this product, the study area is dominated by grasslands $(53 \%)$, croplands $(19 \%)$, and deciduous forest $(13 \%)$. Less frequent land cover types include evergreen forest (3.9\%) and open shrublands (3.5\%) (Luo et al., 2005).

\section{The shape of surface albedo spectrum and correlation between spectral channels}

The land cover classification derived from satellite imagery is usually based on the spectral clustering (Latifovic and Pouliot, 2005). This approach has a long history and has been confirmed by validation of the results using ground and aerial surveys. This indicates that the albedo spectra have similarities in their shapes within the same land cover class under similar environmental conditions and growth phase. In other words, a certain degree of correlation exists among different spectral bands in the surface albedo spectrum for the same land cover type. Several basic albedo curves for various land cover types are well known. These are green vegetation, soil and dry or photosynthetically inactive vegetation, snow/ice, and water (Bowker et al., 1985). For aerosol retrieval from space, the correlation between the shortwave NIR and VIS channels is employed (Kaufman et al., 1997; Kaufman and Remer, 1994). The multispectral surface albedo model tuned to the satellite-observed visible band albedo is used in the ISCCP radiative transfer scheme (Zhang et al., 2004). The coefficients for the albedo model were obtained for 8 land cover types based on a regression with ERBE observations. The method we 
propose is based on a similar concept, that is employing the correlation between channels for a particular land cover type in order to reproduce the shape of the surface reflectance spectrum from a limited spectral input available from historical sensors. The spectral bands for AVHRR, VGT, and MODIS are shown in Figure 1 and the parameters are listed in Table 1.

The successful use of the correlation approach for aerosol retrievals suggests its usefulness for multi-spectral surface albedo retrievals. An example of the correlation among different channels is demonstrated in Figure 4. It shows the scatter plots of MODIS surface spectral albedo for channels 3 to 7 versus albedo in channels 1 and 2 and NDVI of grassland over the ARM SGP area for composite interval May 11-20, 2003. The MODIS albedo was derived as described by Luo et al. (2005). These data are available from the ARM archive (http://www.arm.gov/data/pi_products.stm) and the Canada Centre for Remote Sensing (CCRS) $\mathrm{ftp}$ site (ftp://ftp.ccrs.nrcan.gc.ca/ad/CCRS_ARM/). The $R$-value shown on each panel is the correlation coefficient. The blue channel (B3), green channel (B4), and two SWIR channels (B6, B7) are approximately linearly related to the red channel. The NIR channel (B5) has low correlation with the red channel, but shows some correlation with the other NIR channel (B2). One can also notice that all the channels showing correlation with $B 1$ are less correlated with $B 2$, which means $B 1$ and $B 2$ can be considered, to some degree, as "orthogonal channels", and constitute the basic components in reconstruction of the other channels. The channels $B 3, B 4, B 6$, and $B 7$ show negative correlation with NDVI, and the channel $B 5$ shows quite complex nonlinear relationship with NDVI.

If the land cover data are not taken into account, the degree of correlation between channels decreases, although it can still be quite high. Therefore, a reliable land cover map used as a priori information can be helpful for this purpose. A multiple regression that uses several channels and 
NDVI simultaneously can also improve the results compared to a regression model with one predictor (input channel). Further, Figure 4 suggests that a nonlinear regression model may produce improved results, as the nonlinearity is evident from the scatter plots, especially for channel B5.

\section{Regression models}

The purpose of a regression model is to replicate all seven MODIS channels ( $B 1$ to $B 7$ ) using the four VGT channels or the three AVHRR channels as input. Because the historical AVHRR data do not have the $1.6 \mu \mathrm{m}$ band $B 3 A$, we used the AVHRR bands $B 1$ and $B 2$ and AVHRR $3.7 \mu \mathrm{m}$ band $(B 3 B)$, which was converted to reflectance by subtracting the thermal component as described by Trishchenko (2006). The above AVHRR channels are present on all historical AVHRR sensors. The regression model for converting the four VGT channels into each MODISequivalent channel was designed as

$$
B_{i}^{M O D I S}=a_{0, i}+\left(a_{1, i} B_{0}^{V G T}+a_{2, i} B_{2}^{V G T}+a_{3, i} B_{3}^{V G T}+a_{4, i} B_{4}^{V G T}\right)\left(1+a_{5, i} N D V I^{V G T}+a_{6, i}\left(N D V I^{V G T}\right)^{2}\right)
$$

The regression model for converting the three AVHRR channels into each MODIS-equivalent channel was constructed as

$$
\begin{gathered}
B_{i}^{\text {MODIS }}=a_{0, i}+\left(a_{1, i} B_{1}^{\text {AVHRR }}+a_{2, i} B_{2}^{\text {AVHRR }}+a_{3, i} B_{3 B}^{\text {AVHRR }}\right)\left(1+a_{4, i} N D V I^{\text {AVHRR }}\right)+ \\
a_{5, i}\left(B_{1}^{\text {AVHRR }}\right)^{2}+a_{6, i}\left(B_{2}^{\text {AVHRR }}\right)^{2}+a_{7, i}\left(B_{1}^{\text {AVHRR }} B_{2}{ }^{\text {AVHRR }}\right)
\end{gathered}
$$

where $B_{i}$ could be either the surface albedo or a BRDF parameter, $a_{j, i}$ is the regression model coefficient, $i=1,2, \ldots 7$ denote one of the MODIS channels. Slightly different models were implemented for VGT and AVHRR, because VGT has more channels, and they are better aligned with those from MODIS. The non-linear combination of channels was added for AVHRR regression to reduce the regression residuals and to improve the regression accuracy. 
The coefficients of the regression model were obtained from a large amount of observations from MODIS, VGT, and AVHRR during the overlapping period in 2000/2001. The regression coefficients were derived using the nonlinear least-squares method of Levenberg-Marquardt (Press et al., 1992). To ensure that the linear terms of Eqs (19-20) capture the major features, a stepwise regression was implemented. To start, the linear regression coefficients were retrieved without the nonlinear terms. The coefficients for nonlinear terms were derived by fitting the residual difference between the observations and the linear model. This procedure was repeated until the total normalized residual error became less than $0.1 \%$.

\section{Generation of MODIS-equivalent spectral reflectance from AVHRR and VGT data}

\subsection{Input data}

VGT data. The VGT satellite data used in this study were acquired from the data distribution center of the VEGETATION Programme (http://www.vgt.vito.be). This is a joint initiative of the European Commission, France, Belgium, Sweden and Italy. The distributed 10-day composite surface reflectance dataset (S10) contains the clear-sky pixel observations, corrected for atmospheric effects based on a procedure similar to Rahman and Dedieu (1994). Detailed information about VGT data processing scheme can be found at (http://vegetation.cnes.fr/userguide/userguide.htm). Although a cloud screening procedure had been applied by the data providers, we found that residual cloud contamination still occurred. To address this issue an additional cloud and snow screening algorithm was developed and applied . It uses thresholds for all four VGT channels as well as NDVI. These thresholds were tuned specifically for the SGP area depending on land cover types as described by Luo et al. (2004). The BDRF and albedo maps over 10-day intervals were produced using the land cover-based fitting (LBF) procedure as described by Luo et al. $(2004,2005)$. According to the LBF procedure, 
pixels are grouped together for the same land cover type, and all observations are sorted into small bins of NDVI values. The robust fitting procedure is then applied to obtain the kernel weights $f_{k}(\lambda)$ defined in Eq. (9). This method was implemented in the same way for all sensors over the same territory for regular 10-day intervals, i.e. three intervals per month. The length of the last time interval of the month varies from 8 to 11 days for different months.

AVHRR data. The raw AVHRR data were acquired from the satellite data archive of the Canada Centre for Remote Sensing. The AVHRR data from NOAA-14 spacecraft were used. 10day clear-sky composite surface reflectance data were generated and corrected for the atmospheric effects using the procedures described in Cihlar et al. (2002), Trishchenko et al. (2002b) and Latifovic et al. (2005). The calibration coefficients for channels 1 and 2 proposed by Tahnk and Coakley (2001a; 2001b) for AVHRR/NOAA-14 were employed. In addition to bands $B 1$ and $B 2$, the third spectral band $B 3 B$ centered at $3.75 \mu \mathrm{m}$ was also used. This channel shows a certain degree of sensitivity to snow and to liquid water in the vegetation foliage, which absorbs the solar radiation in the SWIR range (Kaufman and Remer 1994; Khlopenkov and Trishchenko 2007). The inclusion of the AVHRR channel $B 3 B$ in our regression model may serve as a partial compensation for the lack of detailed SWIR information in historical AVHRR data. The BRDF and albedo parameter maps were produced as described in the section related to VGT data processing.

MODIS data. The detailed description of the processing steps and MODIS data used in this study can be found in Luo et al. (2005). The standard MODIS albedo/BRDF products were not used because the time sampling intervals and the output projection were not consistent with the AVHRR and VGT data. The MODIS albedo/BRDF data were produced at $0.5 \mathrm{~km}$ spatial resolution on the spatial grid that was precisely nested within the $1 \mathrm{~km}$ grid used in AVHRR and 
VGT data processing chain. As such, we applied an averaging window of $2 \times 2$ pixels to provide compatibility with the $1 \mathrm{~km}$ spatial data.

Data initial assessment. The regression model described by Eqs (19-20) was designed and implemented for each BRDF parameter $f_{k}(\lambda)$, rather than for the directional reflectances (which vary with the sun-target-view geometry). This approach reduces the effects caused by variable observational geometry and improves the reliability of results.

The time series of the surface albedo and BRDF parameters were produced at regular 10-day time intervals from year 2000 to 2004 for MODIS, from 1998 to 2004 for VGT, and from 1995 to 2001 for AVHRR (see Table 1). They are available from the Canada Centre for Remote Sensing ftp site (ftp://ftp.ccrs.nrcan.gc.ca/ad/CCRS_ARM/). The common spectral bands for the above sensors are the red and NIR, i.e. bands $B 1$ and $B 2$ for AVHRR and MODIS and $B 2$ and $B 3$ for VGT. Figure 5 displays the ARM SGP surface albedo (direct albedo, computed for local solar noon) for these two common bands. The images for all three sensors were derived for the same period: May 1-10, 2000. Although a very high degree of similarity is evident, some differences can be seen. The VGT shows systematically lower albedo in the NIR channel than the MODIS and AVHRR ones. This bias exists for all years.

To better understand the level of agreement, similar channels were compared against each other. Figure 6 shows scatter plots of MODIS and VGT direct local noon albedo for the MODIS $B 3$ vs. VGT $B 0$, MODIS $B 1$ vs. VGT $B 2$, MODIS $B 2$ vs. VGT $B 3$, as well as MODIS B6 vs. VGT $B 4$ channels. The comparison of VGT and AVHRR channels is shown for VGT B2 vs. AVHRR B1, as well as VGT B3 vs. AVHRR B2. Two periods are shown: May 11-20, 2003 and July 21-31, 1998, respectively. Mean bias between two datasets $(\bar{\Delta})$, standard deviation of the bias $(\delta)$, and the correlation coefficient between two datasets $(R)$, are also provided. The 
comparison reveals a high degree of correlation between similar channels, typically around 0.9 and higher, although some biases are possible. The bias for some channels can be up to $15 \%$ (absolute), as can be seen for comparison between MODIS B2 and VGT B3. These biases can be explained, in particular, by the differences in the spectral response functions between channels (Trishchenko et al., 2002a) or some differences in the atmospheric correction approaches. Indepth analysis, however, has revealed that this cannot be explained exclusively by these factors (the SRF effect is less than $3 \%$, the atmospheric correction less than $5 \%$, relative). It can be concluded that the difference may occur due to the issues with sensor's radiometric calibration and the solar constant definitions (up to 10\%) (Luo and Trishchenko, 2005). Nonetheless, the high degree of overall correlation indicates that the regression models (Eqs. 19-20) should be very successful in replicating the MODIS-equivalent spectral albedo and BRDF parameters from the historical AVHRR and VGT data for similar channels and potentially for other channels as well, according to the results shown in Figure 4.

\subsection{Details of implementation}

A one year cycle (March 2000 to February 2001) was used as a baseline period for developing the regression models described by Eqs.(19-20). Data for all the sensors exist for this interval. To reduce the influence of spatial resolution between the sensors and the point spread function, the imagery was aggregated to $2 \mathrm{~km}$ spatial resolution. Regressions were produced for the data grouped by land cover type and month of the year. For wintertime, the dynamics of snow cover was included according to the snow mask derived from the imagery. Snow on short vegetation (e.g. grassland, croplands) and snow on tall vegetation (e.g. forest, shrubs) were considered separately. The robust statistical method was applied to remove possible outliers located within the highest and lowest $5 \%$ of the distribution (Trishchenko, 2002). Due to the 
large size of the area, a very large number of data points $\left(\sim 10^{6}\right)$ were available for analysis. To make it practical, a special sampling procedure was developed and employed that randomly selected 5000 data points for each land cover type. The procedure ensured that points were selected randomly and uniformly across the whole area. The number of data points identified above (5000) was determined through the series of tests to provide a balance between the statistical stability of the results and the computational efficiency. To evaluate the sensitivity of the model coefficients to the data sampling procedure, the code was run five times with different samples of data taken from the same time interval. An example of the retrieved coefficients from each run for the VGT regression model Eq. (19) is presented in Figure 7a. The results for AVHRR Eq. (20) are shown in Figure 7b. The different colors in the two plots represent the different runs. Simulations of the MODIS B1 parameter (isotropic BRDF coefficient) for grasslands in May, 2000 are plotted. It can be seen that the coefficients are quite stable and, therefore, not sensitive to the data sampling procedure.

Once the parameters of the regression model were derived for each land cover type and each month of the year, they were applied to the VGT and AVHRR data to produce the multi-spectral BRDF parameters and albedo maps for the entire period from January 1995 to December 2004. The results were obtained at $1 \mathrm{~km}$ spatial resolution for seven spectral bands compatible with MODIS spectral channels designed for land applications. The regression coefficients to retrieve the isotropic BRDF parameter from VGT data using Eq. (19) for grassland and cropland in May 2001 are listed in Table 2. The correlation coefficients $(R)$ and residual error $(\delta)$ of the fit are also shown. The correlation is very high $(>0.99)$ and the regression error is relatively small, which indicates a computationally successful model performance. The residual errors for cropland are usually greater than those for grassland. This can be explained by less uniform conditions over 
the SGP region in May for cropland than for grassland. Table 2 also demonstrates that the regression coefficients are typically larger for spectrally similar channels, which is in agreement with intuitive physical expectations. The exception occurs for the blue channel $B_{3}^{M O D I S}$ and grassland when the largest coefficient $a_{2}$ corresponds to the VGT red channel. This may happen due to the high correlation between the blue and red channels (Figure 4) especially for relatively uniform surface such as grassland shown in Figure 3.

For MODIS channels that have no analogs among VGT bands, all terms contribute to the regression with comparable amplitudes. The albedo maps for SGP area computed for seven MODIS equivalent channels using the VGT and AVHRR input are shown in Figure 8 for the period of May 11-20, 2000. The panels in Figure 8 show results for bands $B 1$ to $B 7$. The maps display the direct surface albedo computed at local solar noon. The results obtained from MODIS and replicated from VGT and AVHRR observations are shown in corresponding columns. A very good correspondence between results for different sensors can be seen.

Table 3 shows regression coefficients for isotropic BRDF parameters for AVHRR (Eq. (20)) for grassland and cropland in May. Although there are only two input channels in the AVHRR regression model, the residual errors $(\delta)$ are still quite small and comparable to the VGT results. The correlation coefficients $(R)$ are also very high. The AVHRR bands, that are spectrally similar or close to MODIS bands, contribute to the regression with the largest amplitudes. The coefficient $a_{3}$ is generally low for bands $B 1$ to $B 4$. It is greater for SWIR channels $(B 5, B 6$ and B7) especially for cropland land cover types.

The land cover classification used in our study is quite generic. It employs only 10 land cover types listed in Luo et al., (2005), such as cropland, grassland, deciduous, needle leaf, mixed forest etc.. The impact of dynamical changes of land cover state (such as cropland to ploughed 
field conversion and v.v. seen in Figure 3) can be captured successfully by using the vegetation index NDVI, and observed reflectances. As such, we believe that our proposed scheme has a good potential for global applications.

\section{Assessment of replicated MODIS-equivalent historical spectral albedo data}

Comparison of the results for bands $B 1$ (red) and $B 2$ (NIR) shown in Figure 8 reveals that the VGT and AVHRR-based replicas of MODIS channels display a very similar spatial distribution of albedo. The bias for VGT NIR band $B 2$ observed in the original data is removed in the replicated data. An improvement is also observed for AVHRR NIR band B2, where the original reflectance was significantly affected by the spectral response function and by the atmospheric water vapor absorption in the NIR region. As such, the implemented procedure provides an effective normalization of VGT and AVHRR $B 1$ and $B 2$ channels to make them consistent with MODIS observations. Comparison of the statistics (results are not shown) confirms that the MODIS-equivalent reflectances derived from AVHRR and VGT became more consistent. Some variations are still possible due to the differences in satellite overpass time and the clear-sky pixel selection procedures. These differences are attributed to the nature of observations made by different sensors from different platforms with independent data processing, cloud identification and compositing schemes.

For the blue band $B 3$ and SWIR band $B 6$, both VGT and AVHRR instruments show very consistent results among each other and with MODIS, although the AVHRR sensor has no spectrally similar bands. For the green (B4), the NIR band $B 5$, and the SWIR band $B 7$, where no spectrally similar bands exist for VGT and AVHRR, the agreements are also fairly good. Thus, the results confirm that it is possible to replicate all seven spectral channels corresponding to the MODIS land observing bands from the historical observations with a reasonable success, 
provided that synchronous MODIS observations are used for regression model training as described above.

The parameters for the regression models are based on the twelve month overlapping period from March 2000 to February 2001 when data for all the sensors were available. Once derived, these parameters were applied to the other time periods to produce BRDF/albedo properties for the seven MODIS-equivalent bands. To qualitatively assess the performance of this approach, we conducted an image comparison between the real MODIS observations and those replicated from VGT observations for periods not used to define the regression model parameters. Figure 9 shows the spectral albedo maps for bands B4, B5, and $B 7$ for April 21-30, 2003. None of these channels are physically observed by VGT. The results from VGT are fairly consistent with MODIS. Some small local differences can be seen, which may occur due to the differences in compositing schemes. The VGT-based MODIS band B5 albedo map, shows an overall agreement with MODIS in terms of spatial pattern, but is somewhat biased toward smaller values. This difficulty in replication of band B5 stems most likely from its highly non-linear dependence on both visible $(B 1)$ and NIR (B2) reflectances, as evident in Figure 4.

The quantitative numerical assessment is shown in Figure 10a, which displays scatter plots of the MODIS observations versus the VGT-based results for May 11-20, 2003. The mean bias $(\bar{\Delta})$, the standard deviation $(\delta)$, and the correlation coefficient $(R)$ between the two datasets are included in each panel. The results of the linear fit are also shown. It is seen that the points are scattered very close to the $1: 1$ line. Substantial systematic bias observed between similar NIR spectral bands of VGT and MODIS on Figure 6 was successfully corrected. Again, the channel $B 5$ demonstrates the least successful results: lowest correlation coefficient $(\mathrm{R}=0.77)$ and highest scattering $(\delta=0.017)$. It can be seen that all absolute biases are generally low even for $B 2$ and $B 5$. 
Nevertheless, a relative bias (ratio of $\Delta$ to a mean value) is less than $4 \%$ in the worst case. Compared with a $15 \%$ bias shown in Figure 6 for comparison between the NIR channels MODIS $B 2$ and VGT B3, the proposed approach can be considered satisfactory. The relative standard deviation for all channels varies from $3 \%$ to $6 \%$, meaning that our method provides the agreement between the observed and replicated channels at the level close to the radiometric calibration uncertainty (Tahnk and Coakley, 2001 a, b; Xiong, et al., 2002).

The comparison with MODIS data is not possible prior 2000. To assess the level of consistency for the historical period, we compared the results derived from the VGT and AVHRR models. Although this is not a direct validation, it may serve as a valuable comparison and indirect validation, especially if we assume that the VGT model is validated against MODIS observations as discussed above. These results are shown in Figure 10b, which compares AVHRR-based versus VGT-based albedo for channels B1-B7 for period July 21-31, 1998. One can see a very good consistency between the two datasets with the distribution of points close to the 1:1 line. Again, the results for band $B 5$ are not as good as for all other channels. The correlation coefficient $R$ is equal to 0.84 in comparison to values of $R$ between 0.92 and 0.98 for all other channels.

A detailed analysis of the entire 1995 to 2004 period was also conducted. The results for each 10-day interval were assessed. It was found that over $90 \%$ of the cases show similar performance to the results presented in Figures 10a-b. The results for the remaining part have the relative bias within 10\%. Overall results are presented in Figure 11a-b. The panels on the left side depict the area-mean albedo for each spectral band and each sensor. The data for AVHRR cover the period from 1995 to 2000. The data for VGT correspond to 1998-2004 period. MODIS data cover the period 2000-2004. The data are plotted at 10-day temporal resolution. One can see a quite 
satisfactory level of agreement between the sensors. The panels on the right side show the relative mean bias and its standard deviation over the whole region. The relative mean biases (denoted with cross symbols) are mostly within $\pm 3 \%$ for the NIR and SWIR channels (B2, B5, and $B 6$ ), and within $\pm 5 \%$ for the visible channels $(B 1, B 3$, and $B 4)$ and SWIR channel $B 7$. In general, the larger relative bias for the latter group of channels is explained by their smaller albedo (cf. Figure 2). Although the absolute biases can be quite small, the relative biases appear larger due to division by smaller numbers. The relative biases for 10-day intervals tend to be mostly scattered around the zero-line, which means that the overall mean value is close to 0 .

The seasonal cycle of the spectral albedo is well-defined and evident from the graphs shown in Figure 11a-b. It reflects phenological seasonal changes in vegetation and snow cover properties. The red $(B 1)$ and NIR (B2) channels vary in opposite phases. Significant absorption in band $B I$ by chlorophyll in the spring and summer time is correlated with strong reflectance in band $B 2$. The blue channel $B 3$ varies similar to the red channel $B 1$. The seasonal cycle of the amplitude for the green channel $B 4$ albedo looks somewhat similar to other visible channels but is less pronounced and noisier because the albedo values for vegetated and non-vegetated (snowfree) areas are close at these wavelengths. Channel $B 5$ is transitional between NIR and SWIR bands, but its variation is rather similar to the NIR channel $B 2$. The SWIR bands $B 6$ and $B 7$ channels demonstrate the reverse temporal dynamics relative to the NIR channel B2. Their interannual and seasonal cycles are also well pronounced.

Because the mean biases are almost uniformly distributed in the positive and negative areas around the zero-line, and the standard deviation of biases is quite stable with time, we expect that the long-term systematic biases are small. As such, one can consider the spectral albedo for bands $B 1-B 7$ derived from historical sensors as reasonably consistent with the MODIS 
observations and suitable for a number of applications. One possible application may be related to the use of these data by GCMs as a boundary condition or for GCM albedo validations (Wang et al., 2006). The data can also be used for radiation budget studies as a boundary condition for computation of solar radiative fluxes and atmospheric heating rates (Zhang et al., 2004).

Special consideration should be given to the sensor calibration issues. We believe that, in general, the regression techniques can remove the systematic differences between sensors caused by the spectral response function (SRF) effect (Trishchenko et al., 2002a). The SRF effect can be considered as time independent, at least in the first approximation, if no spectral drift is observed or the drift can be neglected. However, the issue of sensor calibration is more complicated. The regression coefficients developed on the basis of a short time interval may not be accurate for other periods if the calibration is time dependent and deviates substantially from the truth. The results presented above for a particular period of time and sensor demonstrate that the temporal trends of calibration parameters for AVHRR and VGT were adequate and lead to results quite consistent with MODIS. The accuracy of absolute values can not be evaluated because the regression was tuned to the MODIS observations. However, the absence of any substantial temporal trends in the intercomparison statistics presented in Figure 11a-b provides an evidence in support of applied time dependent calibration factors (Tahnk and Coakley, 2001 a, b; Hagolle et al., 2004).

\section{Conclusions and discussion}

For accurate determination of the surface and atmosphere solar radiation budget, the multispectral surface albedo and the bi-directional properties are required. Albedo exhibits a complex nonlinear influence on the surface and atmosphere absorbed flux due to multiple reflections. Albedo varies quite substantially with wavelength, time and among land cover types. The 
method is proposed in this paper to derive the multi-spectral surface albedo and the BRDF parameters consistent with the MODIS bands $B 1$ to $B 7$ from the historical AVHRR and VGT data. The method exploits the high degree of correlation between spectral channels for the same land cover types in similar conditions. It can be successfully employed to replicate the albedo and the BRDF parameters for additional spectral channels. A non-linear regression model is proposed that uses three AVHRR or four VGT spectral channels to replicate the seven MODIS spectral channels designed for land applications. The model also takes into account the surface land cover type, normalized difference vegetation index and seasonal cycle. The regression model parameters were derived for the period from March 2000 to February 2001 where the MODIS, AVHRR and VGT data exist simultaneously. The regression parameters were produced for every month and for each land cover type in the Southern Great Plains study area, which encompasses a geographic region of size $8^{\circ}$ in latitude by $10^{\circ}$ in longitude. This region is one of the study areas for the US Department of Energy ARM Program (Ackerman and Stokes, 2003).

The model was employed to generate a 10-year (1995-2004) record of the MODISequivalent spectral albedo and BRDF parameters at 10-day intervals (3 per month) at 1-km spatial resolution. For the MODIS period (since March 2000) the data were produced as described by Luo et al. (2005) at 500m spatial resolution. The accuracy of this product was evaluated from the comparison against the real MODIS observations. It was found that, in general, the degree of correlation between the replicated and the real MODIS data is greater than 0.9 for all channels except for the channel $5(1.2 \mu \mathrm{m})$, where the correlation is in the range of $0.75-0.85$. The systematic relative biases are mostly within $5 \%$ and typically do not exceed $10 \%$. Some systematic differences due to the spectral response function effect and possible differences in calibration approach were also improved. These results confirm that it is possible to replicate 
all seven spectral channels corresponding to the MODIS land observing bands from historical observations with a reasonable success, provided that synchronous MODIS observations are used for regression model training as described above. The method can be potentially applied in the future to maintain a multi-spectral MODIS-equivalent temporal record of the surface albedo/BRDF if MODIS suddenly fails but either AVHRR or VGT observations continue. Because our land cover classification method employed over the ARM SGP region is quite generic, the proposed approach can be used globally as well, although this still needs to be verified.

The proposed regression method is probably one of the simplest solutions to the problem of reconstruction of multi-spectral surface albedo using data with limited spectral coverage. A scientifically more rigorous approach could be developed based on more fundamental physical principles, such as the idea of canopy spectral invariants (Huang et al., 2007; Lewis and Disney, 2007) and canopy structure modeling (Wang et al., 2007). Then, instead of the regression model for BRDF parameters, the spectral properties of the basic elements could be retrieved. Although, this approach seems to be more rigorous, it still needs to be implemented and assessed.

Acknowledgements. This work was conducted at the Canada Centre for Remote Sensing, Department of Natural Resources Canada in Project J35 of the Program on "Enhancing Resilience in a Changing Climate". This study was also supported by the US Department of Energy Atmospheric Radiation Measurements Program grant No. DE-FG02-02ER63351 to CCRS. The MODIS data were acquired through the NASA Distributed Active Archive Center. The VGT data were acquired from distribution center of the VEGETATION Programme (http://www.vgt.vito.be). The authors thank Gunar Fedosejevs, Darren Pouliot for their help with editing and critical review of the manuscript at CCRS. 


\section{References}

Ackerman, T. P., and G. M. Stokes, 2003: The Atmospheric Radiation Measurement Program. Physics Today, 56(1), 38-43.

Ackerman, T. P., D. M. Flynn, and R. T. Marchand, 2003: Quantifying the magnitude of anomalous solar absorption. J. Geophys. Res., 108(D9), 4273, doi:10.1029/2002JD002674.

Barkstrom, B.R., E.F.Harrison, G.L.Smith, R.Green, J.Kibler, R.Cess and the ERBE Science Team, 1989: Earth Radiation Budget Experiment (ERBE) archival and April 1985 results. Bull. Amer. Meteor. Soc., 70, 1254-1262.

Barnsley, M. J., T. Quaife, P. D. Hobson, J. Shaw, P. Lewis, M. Disney, J-P. Muller, A. H. Strahler, C. Barker Schaaf, and W. Lucht, 2000: Estimation of Land-Surface Albedo and Biophysical Properties using SPOT-4 VGT and Semi-Empirical BRDF Models. Proceedings, Vegetation 2000 Meeting, Ispra, Italy (http://vegetation.cnes.fr/vgtprep/vgt2000/barnsley.pdf).

Bowker, D.E., R.E. Davis, D.L. Myrick, K. Stacy, and W.T. Jones, 1985: Spectral reflectances of natural targets for use in remote sensing studies. NASA Reference Publication, 1139.

Briegleb, B. P., P. Minnis, V. Ramanathan, and E. Harrison, 1986: Comparison of regional clearsky albedos inferred from satellite observations and models comparisons. J. Climate Appl. Meteor., 25, 214-226.

Cao, C., M. Weinreb, and H. Xu, 2004, Predicting simultaneous nadir overpasses among polarorbiting meteorological satellites for the intersatellite calibration of radiometers. . J. Atmos. Oceanic Tech., 21, 537- 542.

Chandrasekhar, S., 1960: Radiative transfer. Dover Publications, Inc. 393pp.

Charney, J. G., W. J. Quirk, S.-M. Chow, and J. Kornfield, 1997: A comparative study of the effects of albedo change on drought in semi-arid regions. J. Atmos. Sci., 34, 1366- 1385. 
Cihlar, J., J. M. Chen, Z. Li, R. Latifovic, G. Fedosejevs, M. Adair, W. M. Park, R. Fraser, A. P. Trishchenko, B. Guindon, D. Stanley, and D. Morse, 2002: GeoComp-n, an advanced system for the processing of coarse and medium resolution satellite data. Part 2: Biophysical products for northern ecosystems. Canadian J. Remote Sens., 28, 21-44.

Collins, W.D., P. J. Rasch, B. A. Boville,J.J. Hack, J. R. McCaa, D. L. Williamson, J. T. Kiehl, B.Briegleb , C. Bitz, S.-J. Lin, M. Zhang, Y. Dai, 2004: Description of the NCAR Community Atmosphere Model (CAM 3.0). NCAR/TN-464+STR. NCAR Technical Note. June 2004. Available at http://www.ccsm.ucar.edu/models/atm-cam/docs/description/.

Csiszar, I. and G. Gutman, 1999: Mapping global land surface albedo from NOAA/AVHRR. $J$. Geophys. Res., 104, 6215-6228.

Dickinson, R. E., 1983: Land surface processes and climate-surface albedos and energy balance. Adv. Geophys., 25, 305-353.

Dirmeyer, P. A., and J. Shukla, 1994: Albedo as a modulator of climate response to tropical deforestation. J. Geophys. Res., 99, 20,863-20,878.

Disney, M., P.Lewis, G.Thackrah, T.Quaife, and M.Barnsley, 2004: Comparison of MODIS broadband albedo over an agricultural site with ground measurements and values derived from Earth observation data at a range of spatial scales, Int. J. Remote Sens., 25:23, 5297 5317

Duchemin, B., B. Berthelot, G. Dedieu, M. Leroy, and P.Maisongrande, 2002: Normalization of directional effects in 10-day global syntheses derived from VEGETATION/SPOT: II. Validation of an operational method on actual datasets. Remote Sens. Environ., 81(1), 101113. 
Duchemin, B., and P. Maisongrande, 2002: Normalization of directional effects in 10-day global syntheses derived from VEGETATION/SPOT: I. Investigation of concepts based on simulation, Remote Sens. Environ., 81(1), 90-100

Goodrum, G., K.B.Kidwell, W.Winston, (Eds), 2000: NOAA KLM User's Guide. Revised. US Department of Commerce, NESDIS, NOAA, National Climatic Data Center, Satellite Data Services Division, Washington, DC, USA.[available at http://www2.ncdc.noaa.gov/docs/klm/index.htm]

Guenther, B., G. D. Godden, X. Xiong, E. J. Knight, S. Y. Qiu, H. Montgomery, M. M. Hopkins, M. G. Khayat, and Z. Hao, 1998: Prelaunch algorithm and data format for the Level 1B calibration products for the EOS AM-1 Moderate Resolution Imaging Spectroradiometer (MODIS). IEEE Trans. Geosci. Remote Sens., 36, 1142-1151.

Hagolle, O., J.-M..Nicolas, B.Fougnie, F.Cabot, P. Henry, 2004: Absolute calibration of VEGETATION derived from an interband method based on the sun glint over ocean . IEEE Trans. Geosci. Remote Sens., 42 (7), pp. 1472-1481.

Halthore, R N; D.Crisp, S.E.Schwartz, G.P.Anderson, A.Berk, B.Bonnel, O.Boucher, F.-L. Chang, M.-D.Chou, E.E. Clothiaux, P.Dubuisson, B.A.Fomin, Y.Fouquart, S.Freidenreich, C.Gautier, S.Kato, I.Laszlo, Z.Li, J.H. Mather, A. Plana-Fattori, V.Ramaswamy, P.Richiazzi, Y. Shiren, A.P.Trishchenko, W. Wiscombe, 2005: Intercomparison of shortwave radiative transfer codes and measurements. J. Geophys. Res., D110, 10.1029/2004JD0052932005.

Houghton, J. T., Y. Ding, D. J. Griggs, M. Noguer, P. J. van der Linden, X. Dai, K. Maskell, and C.A. Johnson (Eds.), 2001: Climate Change 2001: The Scientific Basis. Contribution of Working Group I to the Third Assessment Report of the Intergovernmental Panel on Climate Change, Cambridge University Press, New York. 
Huang D., Y.Knyazikhin, R.E.Dickinson, M.Rautiainen, P.Stenberg, M.Disney, P.Lewis, A.Cescatti, Y.Tian, W.Verhoef, J.V.Martonchik, R.B.Myneni, 2007: Canopy spectral invariants for remote sensing and model applications. Remote Sens. Environ., 106, 106-122.

Iacono, MJ , EJ Mlawer, SA Clough, and J-J Morcrette, 2000: Impact of an improved longwave radiation model, RRTM, on the energy budget and thermodynamic properties of the NCAR community climate model, CCM3. J. Geophys. Res., 105, 14,873.

Kandel, R., M. Viollier, P. Raberanto, J.P. Duvel, L.A. Pakhomov, V.A. Golovko, A.P. Trishchenko, J. Mueller, E. Raschke, and R. Stuhlmann, 1998: The ScaRaB earth radiation budget dataset. Bull. Amer. Meteor. Soc., 79, 765-783.

Kaufman, Y. J., D.Tanre, L.A.Remer, E.F.Vermote, A. Chu, A., B.N.Holben, 1997: Operational remote sensing of tropospheric aerosol over the land from EOS-MODIS. J. Geophys. Res, 102(14), 17051-17068.

Kaufman, Y. J., and L. Remer, 1994: Detection of forests using mid-IR reflectance: An application for aerosol studies. IEEE Trans. Geosci. Remote Sens., 32, 672-683.

Khlopenkov, K.V., and A.P. Trishchenko, 2007: SPARC: New cloud, snow, and cloud shadow detection scheme for historical 1-km AVHHR data over Canada. . J. Atmos. Oceanic Tech., $24,322-343$.

Kidwell, K. B. (Editor), 1998, NOAA Polar Orbiter Data User's Guide. US Department of Commerce, NESDIS, NOAA, National Climatic Data Centre, Satellite Data Services Division, Washington, D.C., USA.

Latifovic, R, and D. Pouliot, 2005: Multi-temporal landcover mapping for Canada: methodology and product. Canadian J. Remote Sens., 31(5), 347-363. 
Latifovic, R., Z.-L. Zhu, J. Cihlar, C. Giri, and I. Olthof, 2004: Land cover mapping of North and Central America - Global Land Cover 2000. Remote Sens. Environ., 90, 116-127.

Lewis P., and M. Disney, 2007: Spectral invariants and scattering across multiple scales from within-leaf to canopy. Remote Sens. Environ., 109, 196-206

Li, Z., M. Cribb, A. P. Trishchenko, 2002: Impact of surface inhomogeneity on solar radiative transfer under overcast conditions. J. Geophys. Res. D107(16), AAC 6-1 to AAC 6-15, 2001JD000976

Liang, S., 2003: A direct algorithm for estimating land surface broadband albedos from MODIS imagery, IEEE Trans. Geosci Remote Sens., 41, 136-145.

Liou, K-N., 2002: An Introduction to Atmospheric Radiation. 2nd edition, Academic Press, San Diego, 583 pp. (International Geophysics Series, Volume 84).

Luo, Y., A. P. Trishchenko, R. Latifovic, and Z. Li, 2004: Albedo/BRDF retrievals from VEGETATION 10-day composite data over the ARM SGP area. Second International SPOT/VEGETATION Users Conference, March 24-26, 2004, Antwerp, Belgium.

Luo, Y., A. P. Trishchenko, R. Latifovic, and Z. Li, 2005: Surface bidirectional reflectance and albedo properties derived using a land cover-based approach with Moderate Resolution Imaging Spectroradiometer observations. J. Geophys. Res., 110, D01106, doi:10.1029/2004JD004741.

Luo, Y, and A. P. Trishchenko, 2005: Comparison of atmospheric correction results for AVHRR, VGT and MODIS reflective bands used for land applications, AGU Fall Meeting, 2005. Eos Trans. AGU, 86(52), Fall Meet. Suppl., A23A-0929.

Lyapustin, A., 1999: Atmospheric and geometrical effects on land surface albedo, J. Geophys. Res., 104, 4127-4144. 
Morcrette, J-J., 2002: Assessment of the ECMWF model cloudiness and surface radiation fields at the ARM SGP site. Mon. Wea. Rev., 130, 257-277.

Nicodemus, E. F., 1970: Reflectance nomenclature and directional reflectance and emissivity. Applied Optics, 9, 1474-1475

Press, W. H., S. A. Teukolsky, W. T. Vetterling, and B. P. Flannery, 1992: Numerical Recipes in FORTRAN: The Art of Scientific Computing, 2nd Ed. (pp.675-683), Cambridge University Press, Cambridge.

Rao, C. R. N., and J. Chen, 1996: Post-launch calibration of visible and near-infrared channels of the Advanced Very High Resolution Radiometer on the NOAA-14 spacecraft. Int. J. Remote Sens., 17, 2743-2747.

Rahman, H., and G. Dedieu, 1994: SMAC: a simplified method for the atmospheric correction of satellite measurements in the solar spectrum. Int. J. Remote Sens., 15, 123-143.

Rutan, D., and T.P. Charlock, 1997: Spectral reflectance, directional reflectance, and broadband albedo of the earth's surface. 9-th Conference on Atmospheric Radiation, Long Beach, CA, pp.466-470.

Samain, O., B.Geiger, J.-L. Roujean, 2006: Spectral normalization and fusion of optical sensors for the retrieval of BRDF and albedo: Application to VEGETATION, MODIS and MERIS data sets. IEEE Trans. Geosci. Remote Sens., 44 (11), 3166-3178.

Samain, O., J.-L. Roujean, B.Geiger, 2005: Fusion of AVHRR and VEGETATION data using a Kalman filter for the retrieval of surface BRDF and albedo. Proceedings of International Geoscience and Remote Sensing Symposium (IGARSS). v.2., pp.1142-1145. 
Salomonson, V. V., W.L.Barnes, P.W. Maymon, H,E, Montgomery, H.Ostrow, 1989: MODIS: Advanced facility instrument for studies of the earth as a system. IEEE Trans. Geosci. Remote Sens., 27(2), 145-153

Schaaf, C. B., F. Gao, A. H. Strahler, W. Lucht, X. Li, T. Tsang, N. C. Strugnell, X. Zhang, Y. Jin, J.-P. Muller, P. Lewis, M. Barnsley, P. Hobson, M. Disney, G. Roberts, M. Dunderdale, C. Doll, R. d'Entremont, B. Hu, S. Liang, and J. L. Privette, 2002: First Operational BRDF, Albedo and Nadir Reflectance Products from MODIS, Remote Sens. Environ., 83, 135-148.

Stephens, G.L., 1978: Radiation profiles in extended water clouds. Part.I: Theory. J. Atmos. Sci., 35, 2111-2122.

Strahler, A.H., W.Lucht, C.B.Schaaf,T.Tsang, F.Gao, X. Li,J-P. Muller, P. Lewis, M.J.Barnsley, 1999: MODIS BRDF/Albedo Product. Algorithm Theoretical Basis Document. Version 5.0. 53pp. Available at [http://modis.gsfc.nasa.gov/data/atbd/atbd_mod09.pdf].

Strugnell, N., W. Lucht, and C. Schaaf, 2001: A global albedo data set derived from AVHRR data for use in climate simulations. Geophys. Res. Let., 28, 191-194.

Tahnk, W. R., and J. A. Coakley, 2001a: Improved calibration coefficients for NOAA-14 AVHRR visible and near-infrared channels. Int. J. Remote Sens., 22, 1269-1283.

Tahnk, W. R., and J. A. Coakley, 2001b: Updated calibration coefficients for NOAA-14 AVHRR Channels 1 and 2. Int. J. Remote Sens., 22, 3053-3057.

Trishchenko, A.P., K.V. Khlopenkov, C.Ungureanu, R.Latifovic, Y.Luo, W.B.Park, 2007: Mapping of surface albedo over Mackenzie River basin area from satellite observations. In "Atmospheric Dynamics of a Cold Region: the Mackenzie GEWEX Study Experience Volume I". Springer. 20pp. In press. 
Trishchenko, AP, Y.Luo, R. Latifovic, and Z Li, 2004: Land Cover Type Distribution Over the ARM SGP Area for Atmospheric Radiation and Environmental Research. Proc. $14^{\text {th }}$ Science Team Meeting. Albuquerque, NM. $11 \mathrm{pp}$ Available at http://www.arm.gov/publications/proceedings/conf14/extended abs/trishchenko3-ap.pdf

Trishchenko, A. P., J. Cihlar, and Z. Li, 2002a: Effects of spectral response function on the surface reflectance and NDVI measured with moderate resolution sensors. Remote Sens. Environ., 81, 1-18.

Trishchenko, A.P, B.Hwang, Z Li, 2002b: Atmospheric correction of satellite signal in solar domain: Impact of improved molecular spectroscopy. Proc. 12th ARM Science Team Meeting, 7pp. (available at http://www.arm.gov/docs/documents/technical/conf 0204 /trishchenkoap.pdf)

Trishchenko, A. P., 2002: Removing unwanted fluctuations in the AVHRR thermal calibration data using robust techniques. J. Atmos. Oceanic Tech., 19, 1939-1954.

Trishchenko, A. P., 2006: Solar irradiance and brightness temperatures of AVHRR and GOES SW channels. J. Atmos. Oceanic Tech.., 23, pp.198-210.

Wang, S., A.P.Trishchenko, X.Sun, 2007: Simulation of canopy radiation transfer and surface albedo in the EALCO model. Climate Dynamics. 10.1007/s00382-007-0252-y.

Wang, S., A.P.Trishchenko, K.V.Khlopenkov, A.Davidson, 2006: Comparison of IPCC AR4 climate model simulations of surface albedo with satellite products over Northern Latitudes. $J$. Geophys. Res. 111: D21108, doi:10.1029/2005JD006728.

Wielicki, B.A., B.R. Barkstrom, E.F.Harrison, R.B.Lee, G.L. Smith, J.E.Cooper, 1996: Clouds and the Earth's Radiant Energy System (CERES): An Earth Observing System Experiment. Bull. Amer. Meteor. Soc., 77, 853-868 
Xiong, X., K. Chiang, B. Guenther, and W. L. Barnes, 2002: MODIS reflective solar bands calibration algorithm and on-orbit performance. Proceedings SPIE, Optical Remote Sensing of the Atmosphere and Clouds III, Vol.4891.

Xiong, X., Barnes, W. 2006: An overview of MODIS radiometric calibration and characterization. Advances in Atmos. Sci., 23(1), pp. 69-79. doi: 10.1007/s00376-006-0008-3.

Zhang, Y., W. B. Rossow, A. A. Lacis, V. Oinas, and M. I. Mishchenko, 2004: Calculation of radiative fluxes from the surface to top of atmosphere based on ISCCP and other global data sets: Refinements of the radiative transfer model and the input data, J. Geophys. Res., 109, D19105, doi:10.1029/2003JD004457. 
Table 1. Band name, spectral range, and time period for various sensors used in this study

\begin{tabular}{|c|c|c|c|c|c|c|c|c|}
\hline $\begin{array}{c}\text { Terra/ } \\
\text { MODIS }\end{array}$ & $\begin{array}{l}\text { B1 } \\
(\mathrm{nm})\end{array}$ & $\begin{array}{l}\text { B2 } \\
(\mathrm{nm})\end{array}$ & $\begin{array}{l}\text { B3 } \\
(\mathrm{nm})\end{array}$ & $\begin{array}{l}\text { B4 } \\
(\mathrm{nm})\end{array}$ & $\begin{array}{c}\text { B5 } \\
(\mathrm{nm})\end{array}$ & $\begin{array}{c}\text { B6 } \\
(\mathrm{nm})\end{array}$ & $\begin{array}{c}\text { B7 } \\
(\mathrm{nm})\end{array}$ & Period \\
\hline & $620-670$ & $841-876$ & $459-479$ & $545-565$ & $1230-1250$ & $1628-1652$ & $2105-2155$ & $\begin{array}{l}2000 / 3- \\
2004 / 12\end{array}$ \\
\hline $\begin{array}{l}\text { NOAA-14/ } \\
\text { AVHRR }\end{array}$ & $\begin{array}{l}\text { B1 } \\
(n m)\end{array}$ & $\begin{array}{l}\text { B2 } \\
\text { (nm) }\end{array}$ & & & & $\begin{array}{l}\text { B3 } \\
(\mathrm{nm})\end{array}$ & & \\
\hline & $580-680$ & $725-1100$ & & --- & --- & $\begin{array}{l}\text { B3A: } 1580-1640^{*} \\
\text { B3B: } 3550-3930\end{array}$ & --- & $\begin{array}{l}1995 / 01 \\
-2001 / 3\end{array}$ \\
\hline $\begin{array}{c}\text { SPOT-4,5/ } \\
\text { VGT }\end{array}$ & $\begin{array}{c}\text { B2 } \\
(\mathrm{nm})\end{array}$ & $\begin{array}{c}\text { B3 } \\
(\mathrm{nm})\end{array}$ & $\begin{array}{c}\text { B0 } \\
(\mathrm{nm})\end{array}$ & & & $\begin{array}{c}\text { B4 } \\
(\mathrm{nm})\end{array}$ & & \\
\hline & $610-680$ & $790-890$ & $430-470$ & & --- & $1580-1750$ & --- & $\begin{array}{l}1998 / 4- \\
2004 / 12\end{array}$ \\
\hline
\end{tabular}


Table 2. The regression model coefficients for converting BRDF isotropic parameter of VGT bands to MODIS-equivalent spectral bands.

\begin{tabular}{|c|c|c|c|c|c|c|c|c|c|}
\hline & $a_{0}$ & $a_{1}$ & $a_{2}$ & $a_{3}$ & $a_{4}$ & $a_{5}$ & $a_{6}$ & $R$ & $\delta$ \\
\hline \multicolumn{10}{|c|}{ Grassland in May } \\
\hline$B_{1}^{M O D I S}$ & 0.0046 & -0.2241 & 1.0431 & -0.0784 & 0.1042 & 0.0259 & -0.0483 & 0.9950 & 0.0089 \\
\hline$B_{2}^{M O D I S}$ & 0.0887 & -0.7260 & 0.3276 & 0.9126 & -0.1483 & -0.1274 & 0.1947 & 0.9978 & 0.0219 \\
\hline$B_{3}^{M O D I S}$ & 0.0050 & 0.0301 & 0.4174 & -0.0460 & 0.0603 & 0.0373 & -0.0657 & 0.9931 & 0.0052 \\
\hline$B_{4}^{M O D I S}$ & 0.0201 & 0.0957 & 0.4070 & 0.0280 & 0.0816 & 0.0006 & -0.0023 & 0.9972 & 0.0066 \\
\hline$B_{5}^{M O D I S}$ & 0.1425 & -0.6426 & 0.1753 & 0.4868 & 0.3488 & -0.0284 & 0.0441 & 0.9988 & 0.0185 \\
\hline$B_{6}^{M O D I S}$ & 0.1253 & -0.0564 & 0.5701 & -0.1602 & 0.6803 & 0.0263 & -0.0414 & 0.9983 & 0.0177 \\
\hline$B_{7}^{M O D I S}$ & 0.0309 & -0.2543 & 1.4588 & -0.2214 & 0.3810 & 0.0210 & -0.0353 & 0.9947 & 0.0185 \\
\hline \multicolumn{10}{|c|}{ Cropland in May } \\
\hline$B_{1}^{M O D I S}$ & 0.0280 & 0.1288 & 0.9366 & -0.0962 & 0.0339 & 0.0309 & -0.0378 & 0.9931 & 0.0142 \\
\hline$B_{2}^{M O D I S}$ & 0.0758 & 0.0109 & -0.1659 & 0.8329 & 0.0726 & 0.0005 & -0.0009 & 0.9979 & 0.0203 \\
\hline$B_{3}^{M O D I S}$ & 0.0173 & 0.4708 & 0.2183 & -0.0462 & 0.0301 & 0.0693 & -0.1224 & 0.9904 & 0.0082 \\
\hline$B_{4}^{M O D I S}$ & 0.0363 & 0.4564 & 0.3165 & -0.0100 & 0.0430 & 0.0723 & -0.1376 & 0.9947 & 0.0103 \\
\hline$B_{5}^{M O D I S}$ & 0.1552 & -0.1942 & -0.0359 & 0.3295 & 0.4639 & 0.0661 & -0.1227 & 0.9981 & 0.0228 \\
\hline$B_{6}^{M O D I S}$ & 0.1473 & 0.3309 & 0.3451 & -0.2141 & 0.6727 & 0.0382 & -0.0597 & 0.9965 & 0.0278 \\
\hline$B_{7}^{M O D I S}$ & 0.0576 & 0.1719 & 1.2079 & -0.2523 & 0.3857 & 0.0031 & 0.0055 & 0.9918 & 0.0310 \\
\hline
\end{tabular}


Table 3. The regression model coefficients for converting BRDF isotropic parameter of AVHRR bands into MODIS-equivalent bands.

\begin{tabular}{|c|c|c|c|c|c|c|c|c|c|c|}
\hline & $a_{0}$ & $a_{1}$ & $a_{2}$ & $A_{3}$ & $a_{4}$ & $a_{5}$ & $a_{6}$ & $a_{7}$ & $R$ & $\delta$ \\
\hline \multicolumn{11}{|c|}{ Grassland in May } \\
\hline$B_{1}^{M O D I S}$ & 0.0632 & 1.0666 & -0.2086 & -0.0167 & -0.0225 & 1.2337 & 0.0861 & -0.6817 & 0.9948 & 0.0090 \\
\hline$B_{2}^{M O D I S}$ & 0.0953 & -0.7319 & 0.8831 & 0.1418 & 0.0008 & 4.4941 & 0.3310 & -2.5778 & 0.9988 & 0.0164 \\
\hline$B_{3}^{M O D I S}$ & 0.0345 & 0.5181 & -0.1113 & -0.0129 & -0.0143 & 0.3107 & 0.0215 & -0.1717 & 0.9949 & 0.0044 \\
\hline$B_{4}^{M O D I S}$ & 0.0611 & 0.5628 & -0.0597 & -0.0367 & -0.0043 & 0.4067 & 0.0311 & -0.2356 & 0.9984 & 0.0049 \\
\hline$B_{5}^{M O D I S}$ & 0.2411 & 0.0558 & 0.3700 & 0.1183 & 0.0002 & 2.4181 & 0.1826 & -1.4029 & 0.9996 & 0.0102 \\
\hline$B_{6}^{M O D I S}$ & 0.2937 & 1.5549 & -0.3796 & -0.0402 & -0.0123 & 0.9127 & 0.0628 & -0.5069 & 0.9986 & 0.0160 \\
\hline$B_{7}^{M O D I S}$ & 0.1603 & 1.9142 & -0.4458 & -0.0573 & -0.0146 & 1.2438 & 0.0849 & -0.6851 & 0.9959 & 0.0162 \\
\hline \multicolumn{11}{|c|}{ Cropland in May } \\
\hline$B_{1}^{M O D I S}$ & 0.0556 & 0.9470 & -0.1495 & 0.0166 & -0.0020 & 0.1426 & 0.0192 & -0.1099 & 0.9959 & 0.0108 \\
\hline$B_{2}^{M O D I S}$ & 0.1222 & -0.0789 & 0.6176 & 0.0090 & 0.0006 & 1.0578 & 0.1284 & -0.7848 & 0.9988 & 0.0156 \\
\hline$B_{3}^{M O D I S}$ & 0.0385 & 0.3675 & -0.0778 & 0.0112 & 0.0318 & -0.5276 & -0.0620 & 0.3784 & 0.9952 & 0.0056 \\
\hline$B_{4}^{M O D I S}$ & 0.0653 & 0.4745 & -0.0661 & 0.0118 & 0.0097 & -0.4673 & -0.0570 & 0.3433 & 0.9977 & 0.0067 \\
\hline$B_{5}^{M O D I S}$ & 0.2467 & 0.4893 & 0.1716 & 0.0946 & -0.0004 & 0.4520 & 0.0551 & -0.3347 & 0.9993 & 0.0134 \\
\hline$B_{6}^{M O D I S}$ & 0.2670 & 1.2374 & -0.2876 & 0.1463 & 0.0116 & -0.5962 & -0.0684 & 0.4215 & 0.9983 & 0.0196 \\
\hline$B_{7}^{M O D I S}$ & 0.1414 & 1.6270 & -0.3229 & 0.1060 & 0.0034 & -0.3006 & -0.0345 & 0.2134 & 0.9965 & 0.0198 \\
\hline
\end{tabular}




\section{Figure Captions}

Figure 1. Spectral Response Functions (SRF) for (a) Terra-MODIS, (b) SPOT-4 VGT, and (c) NOAA-16 AVHRR sensors (black curves in each plot). Atmospheric transmission for clear-sky (red curves) and overcast $(\mathrm{St}, \tau=20)$ conditions (gray curves). Sample surface spectral albedo for (a) grasslands/cropland (green and dark-green curves), (b) deciduous forest (purple curve) and needle leaf forest (pink and brown curves), and (c) barren land (dark-cyan curve), low-density vegetation (dark-yellow curve), and snow (blue curve).

Figure 2. The SZA dependence of the surface albedo on the sky conditions for different surface cover types: a) ocean, b) grassland, c) forest. Open squares denote clear-sky, black triangles denote Sc cloud with $\tau=5$, crosses denote Sc cloud with $\tau=100$.

Figure 3. Landsat-7 ETM+ images and land cover distribution for the area of $10 \times 10 \mathrm{~km}^{2}$ around the ARM SGP Central Facility $\left(36.607^{\circ} \mathrm{N}, 97.486^{\circ} \mathrm{W}\right)$. a) False color imagery, September 1, 2002; (b) False color imagery, April 13, 2003; (c) Landcover map for September 2002; (d) Land cover map for May 2003.

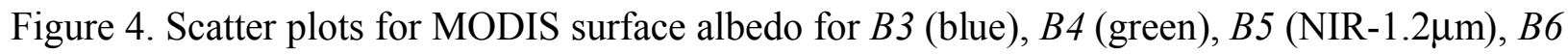

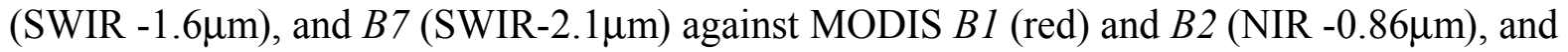
vegetation index NDVI. The data are for composite interval May 11-20, 2003. The $R$-value denotes the coefficient of linear correlation between the two datasets. 
Figure 5. Surface albedo for the extended ARM SGP region of $8^{\circ} \times 10^{\circ}$ for composite interval May 1-10, 2000. Upper panels are for AVHRR, middle panels are for VGT, and bottom panels are for MODIS. Left panels correspond to the red channel, right panels correspond to NIR $(0.86 \mu \mathrm{m})$.

Figure 6. Scatter plots for the MODIS surface albedo versus the VGT at the blue, red, NIR $(0.8 \mu \mathrm{m})$, and one SWIR $(1.6 \mu \mathrm{m})$ channels, and for the VGT versus the AVHRR at the red and NIR $(0.8 \mu \mathrm{m})$ channels. The MODIS vs. VGT data are for composite interval May 11-20 of 2003. The VGT vs. AVHRR data are for composite interval July 21-31 of 1998. The mean bias between the two datasets $(\bar{\Delta})$, the standard deviation of the bias $(\delta)$, and the correlation coefficient of the two datasets $(R)$ are also provided. Values of direct albedo at local solar noon are plotted.

Figure 7. Regression model coefficients $\left(a_{0}, a_{1}, \ldots\right)$ retrieved in five runs with independent random sampling. Regression model is for MODIS-equivalent channel B1 BRDF isotropic parameter for grassland and 10 day composite interval of May, 11-20, 2001. a) VGT model, Eq. (19); b) AVHRR model, Eq. (20).

Figure 8. Surface albedo for seven spectral bands $B 1-B 7$ over the ARM SGP region derived from MODIS, VGT, and AVHRR observations for composite interval May 11-20, 2000. Direct albedo at local solar noon is shown. 
Figure 9. Assessment of surface albedo over the ARM SGP region observed from MODIS and VGT-based replicates for independent composite period April 21-30, 2003. Spectral channels $B 4, B 5$ and $B 7$ are shown.

Figure 10. a) Scatter plots for the MODIS versus the VGT-based surface albedo for seven bands for composite interval May 11-20, 2003. b) the same as a) but for the AVHRR-based versus the VGT-based albedo for composite interval July 21-30, 1998. The mean bias between the two datasets $(\bar{\Delta})$, the standard deviation of the bias $(\delta)$, and the correlation coefficient $(R)$ are shown.

Figure 11. Overall surface albedo statistics for the extended ARM SGP region derived from the AVHRR (1995-2000), VGT (1998-2004), and MODIS/Terra (2000-2004) data at 10-day intervals. Left panels show mean albedo, right panels show relative mean bias (cross symbols) and standard deviation of bias (square symbols). a) bands $B 1-B 3$. b) bands $B 4-B 7$. 


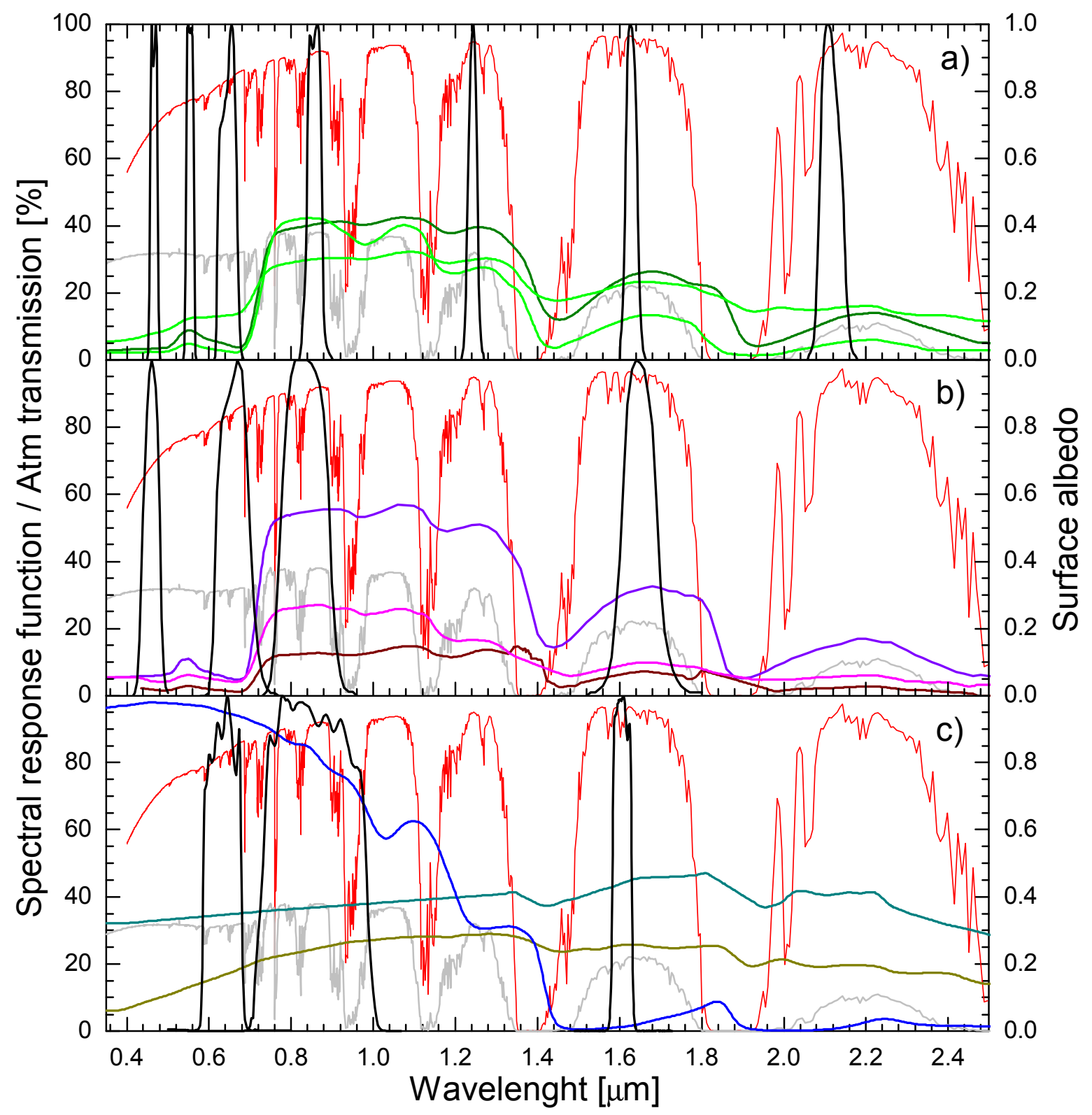

Figure 1. Spectral Response Functions (SRF) for (a) Terra-MODIS, (b) SPOT-4 VGT, and (c) NOAA-16 AVHRR sensors (black curves in each plot). Atmospheric transmission for clear-sky (red curves) and overcast (St, $\tau=20$ ) conditions (gray curves). Sample surface spectral albedo for (a) grasslands/cropland (green and dark-green curves), (b) deciduous forest (purple curve) and needle leaf forest (pink and brown curves), and (c) barren land (dark-cyan curve), low-density vegetation (dark-yellow curve), and snow (blue curve). 


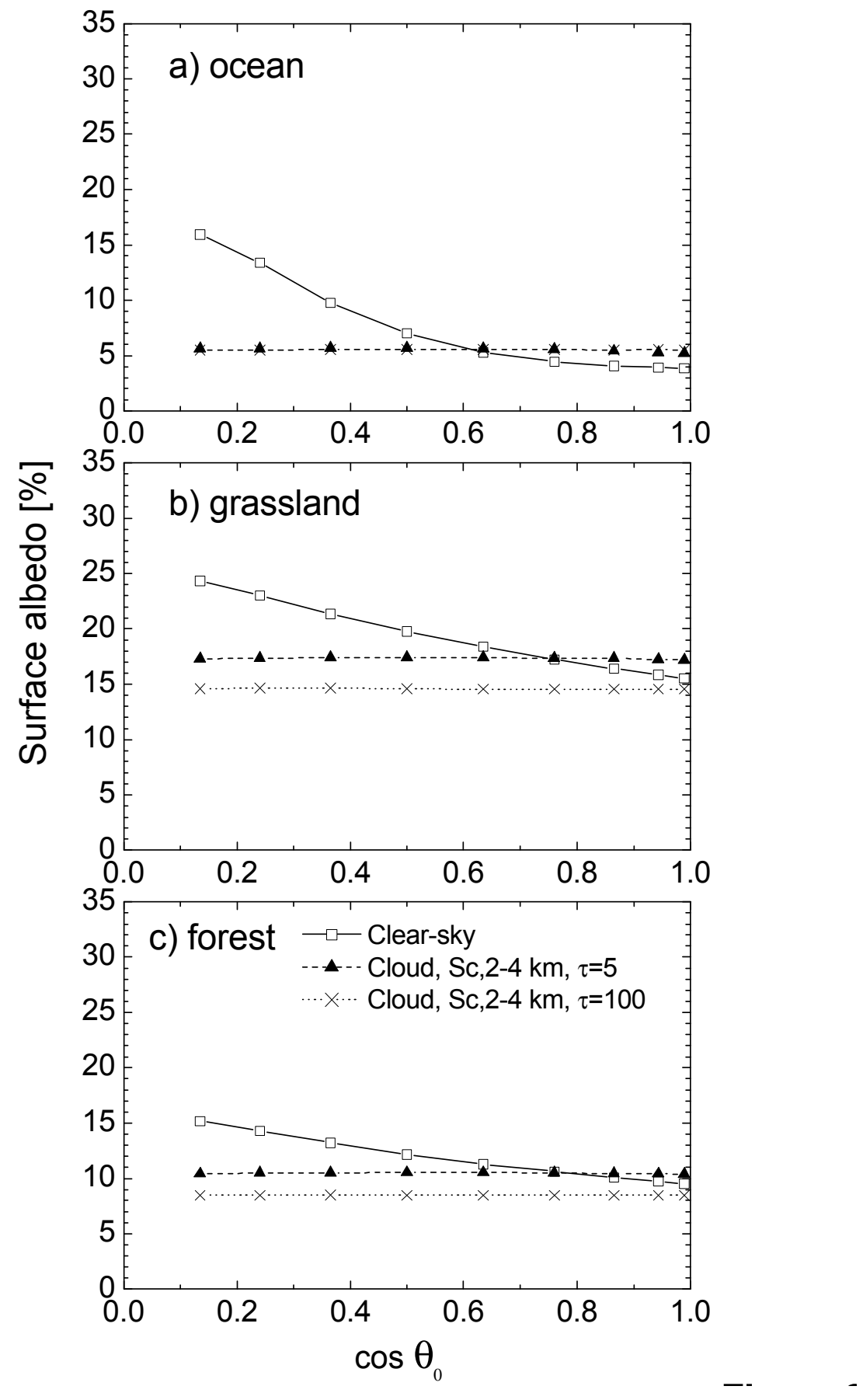

Figure 2. The SZA dependence of the surface albedo on the sky conditions for different surface cover types: a) ocean, b) grassland, c) forest. Open squares denote clear-sky, black triangles denote Sc cloud with $\tau=5$, crosses denote Sc cloud with $\tau=100$. 
(a) Landsat-7 RGB image, 09/01/2002

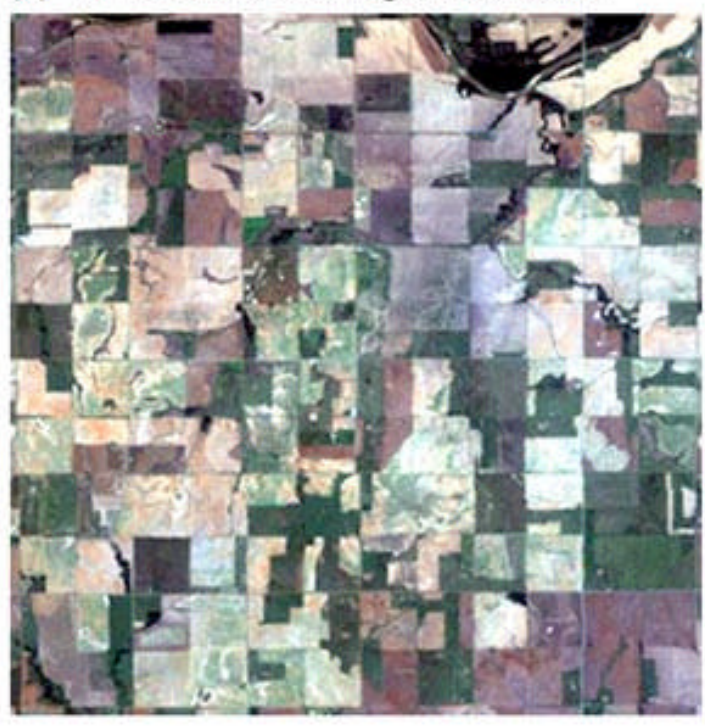

(c) Landcover map, September 2002

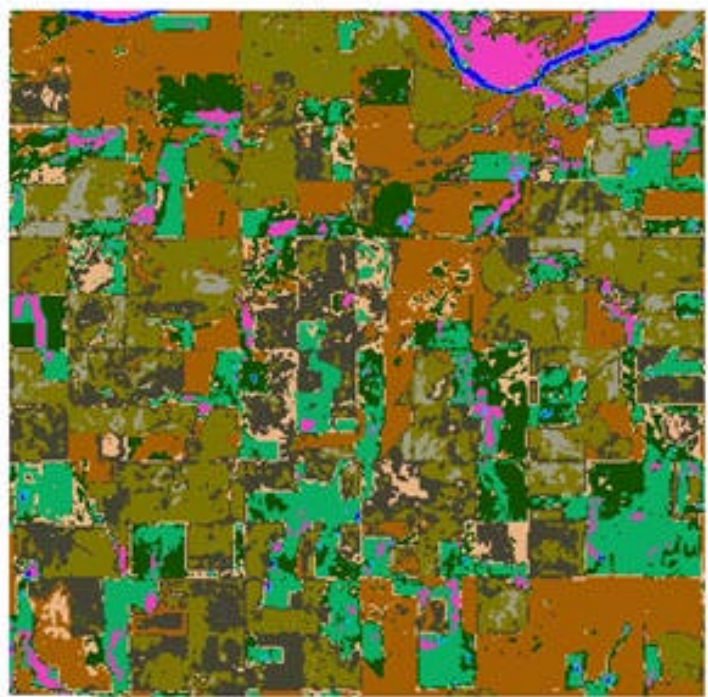

(b) Landsat-7 RGB image, 04/13/2003

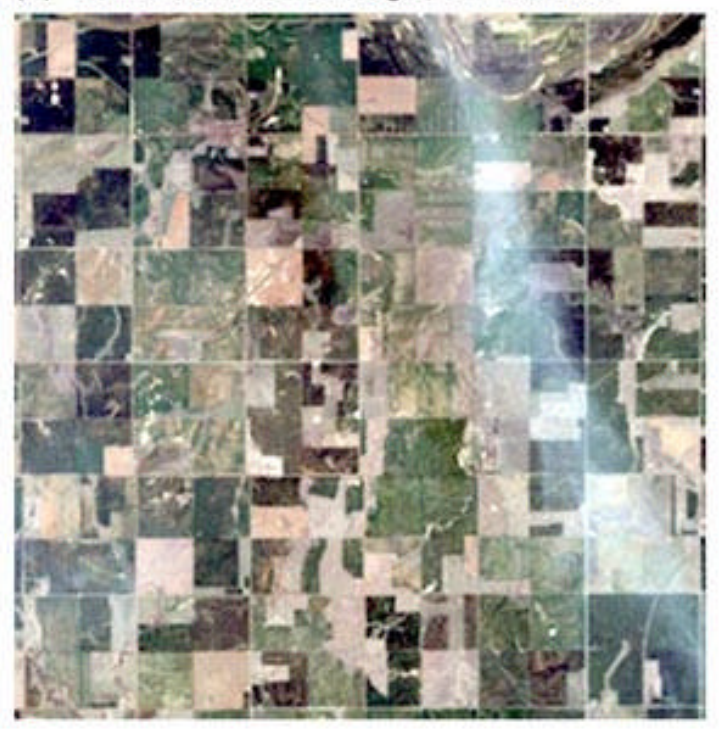

(d) Landcover map, May 2003

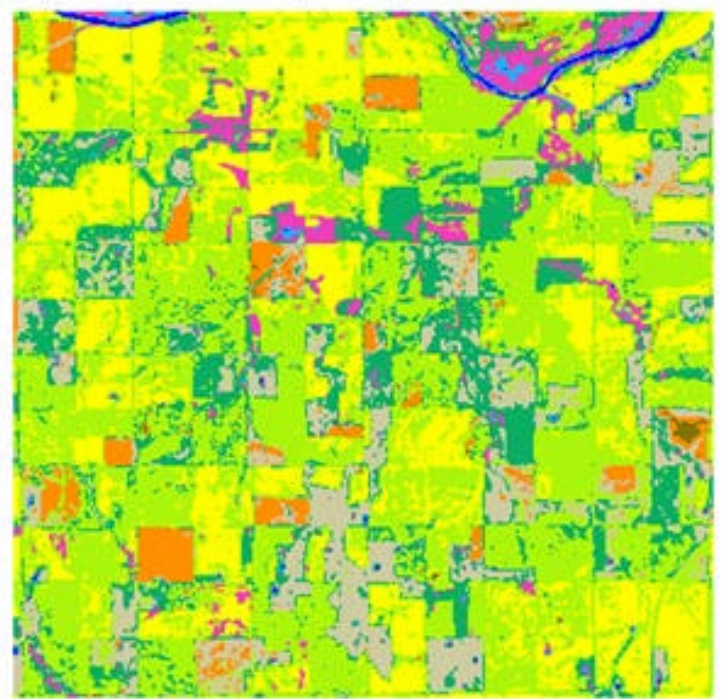

Water

Netland

Tree/shrub

Grass (green)

Corn/milo

Wheat stub/grass (dry)

Baresoil (short grass)

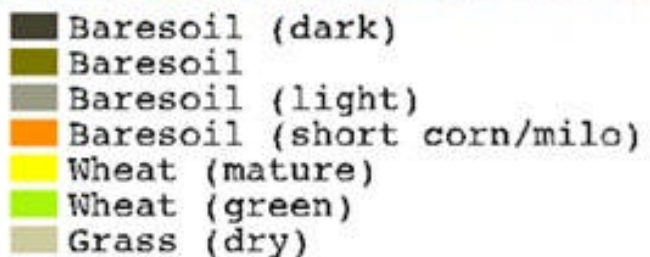

Figure 3. Landsat-7 ETM+ images and land cover distribution for the area of $10 \times 10 \mathrm{~km}^{2}$ around the ARM SGP Central Facility $\left(36.607^{\circ} \mathrm{N}, 97.486^{\circ} \mathrm{W}\right)$. a) False color imagery, September 1, 2002; (b) False color imagery, April 13, 2003; (c) Landcover map for September 2002; (d) Land cover map for May 2003. 

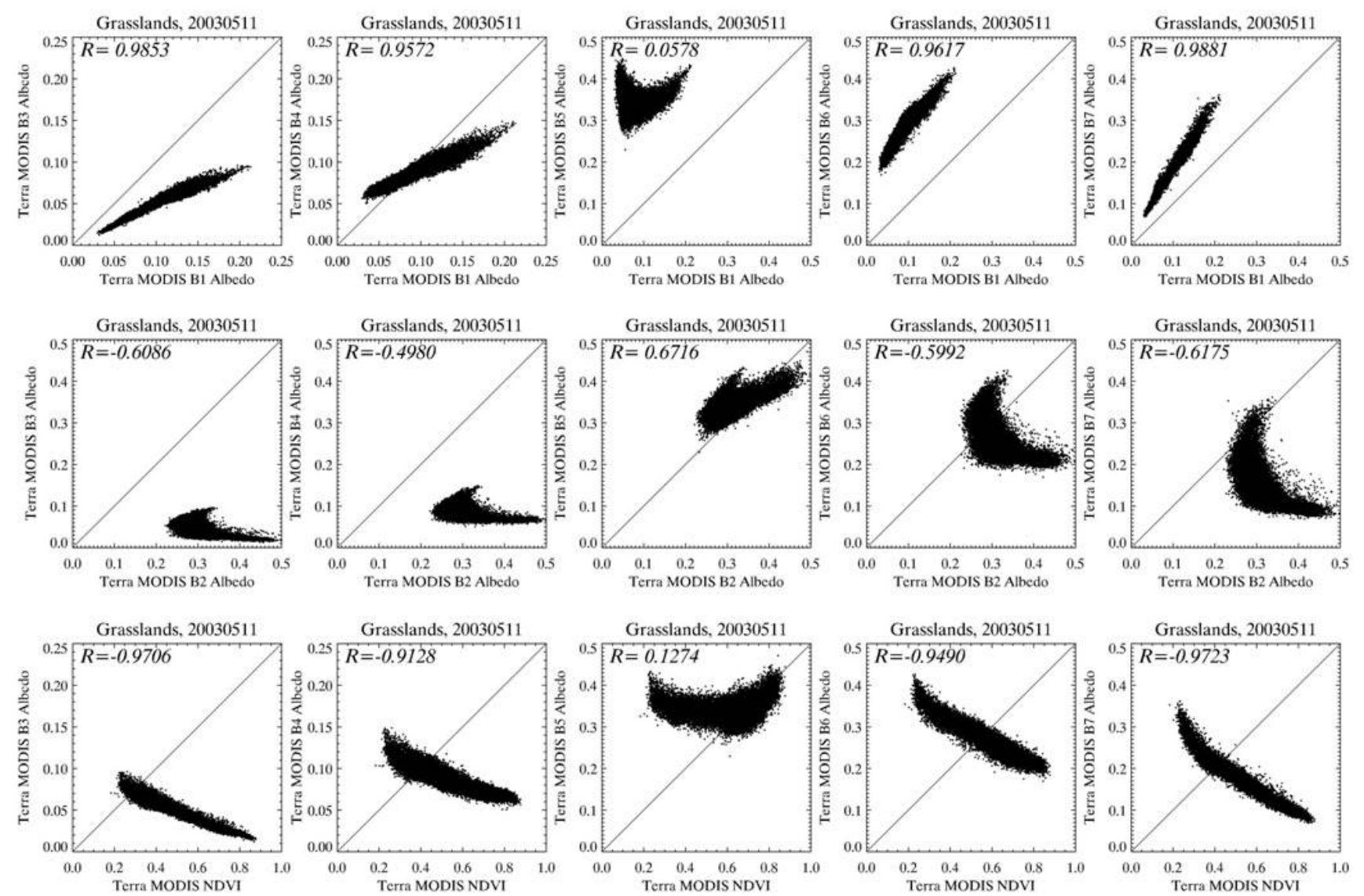

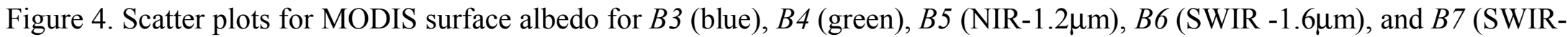

2.1 $\mu \mathrm{m}$ ) against MODIS $B 1$ (red) and $B 2$ (NIR $-0.86 \mu \mathrm{m}$ ), and vegetation index NDVI. The data are for composite interval May 11-20, 2003. The $R$-value denotes the coefficient of linear correlation between the two datasets. 


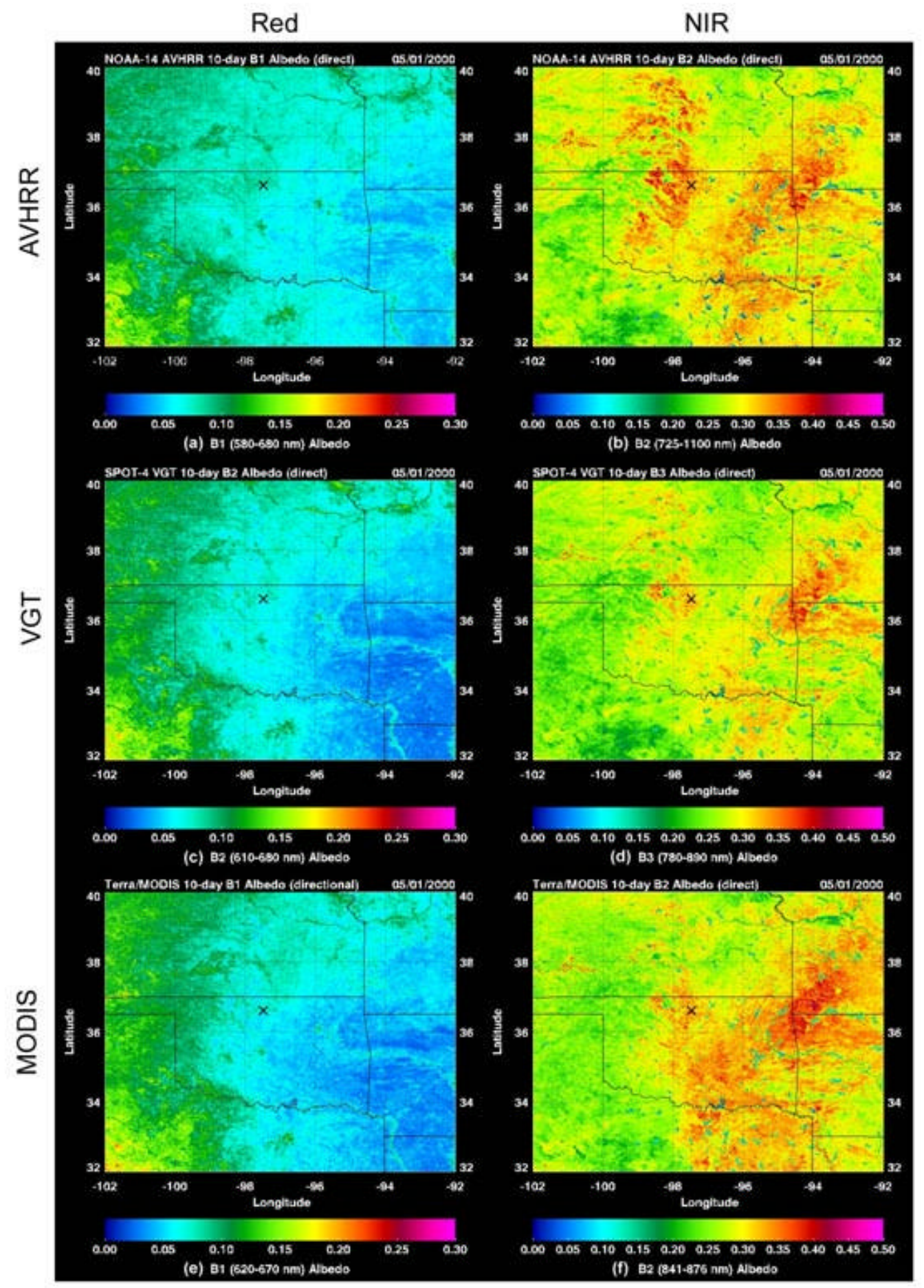

Figure 5. Surface albedo for the extended ARM SGP region of $8^{\circ} \times 10^{\circ}$ for composite interval May 1-10, 2000. Upper panels are for AVHRR, middle panels are for VGT, and bottom panels are for MODIS. Left panels correspond to the red channel, right panels correspond to NIR $(0.86 \mu \mathrm{m})$. 
MODIS vs. VGT
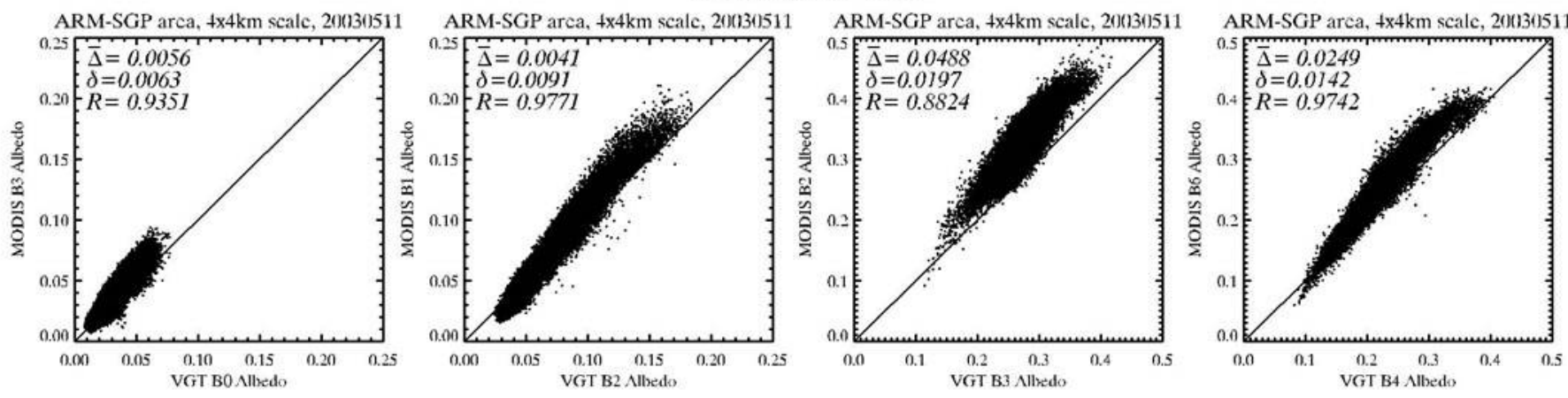

VGT vs. AVHRR

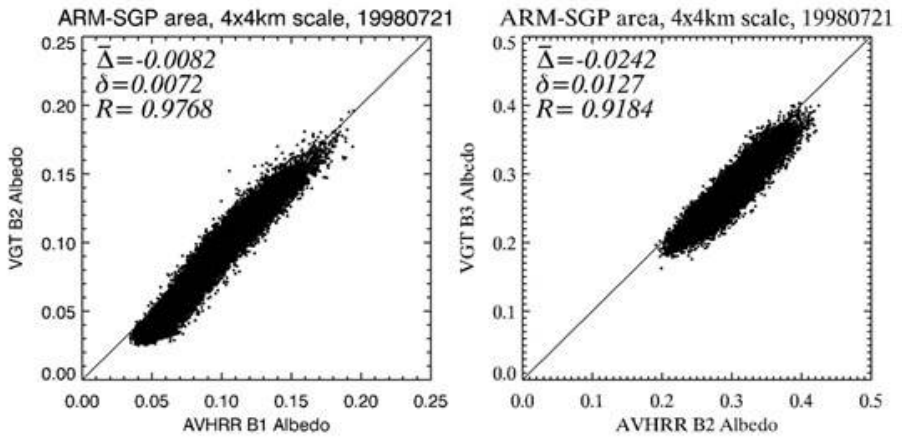

Figure 6. Scatter plots for the MODIS surface albedo versus the VGT at the blue, red, NIR (0.86 $\mu m)$, and one SWIR (1.6 $\mu m)$

channels, and for the VGT versus the AVHRR at the red and NIR $(0.86 \mu \mathrm{m})$ channels. The MODIS vs. VGT data are for composite interval May 11-20 of 2003. The VGT vs. AVHRR data are for composite interval July 21-31 of 1998. The mean bias between the two datasets $(\bar{\Delta})$, the standard deviation of the bias $(\delta)$, and the correlation coefficient of the two datasets (R) are also provided. Values of direct albedo at local solar noon are plotted. 

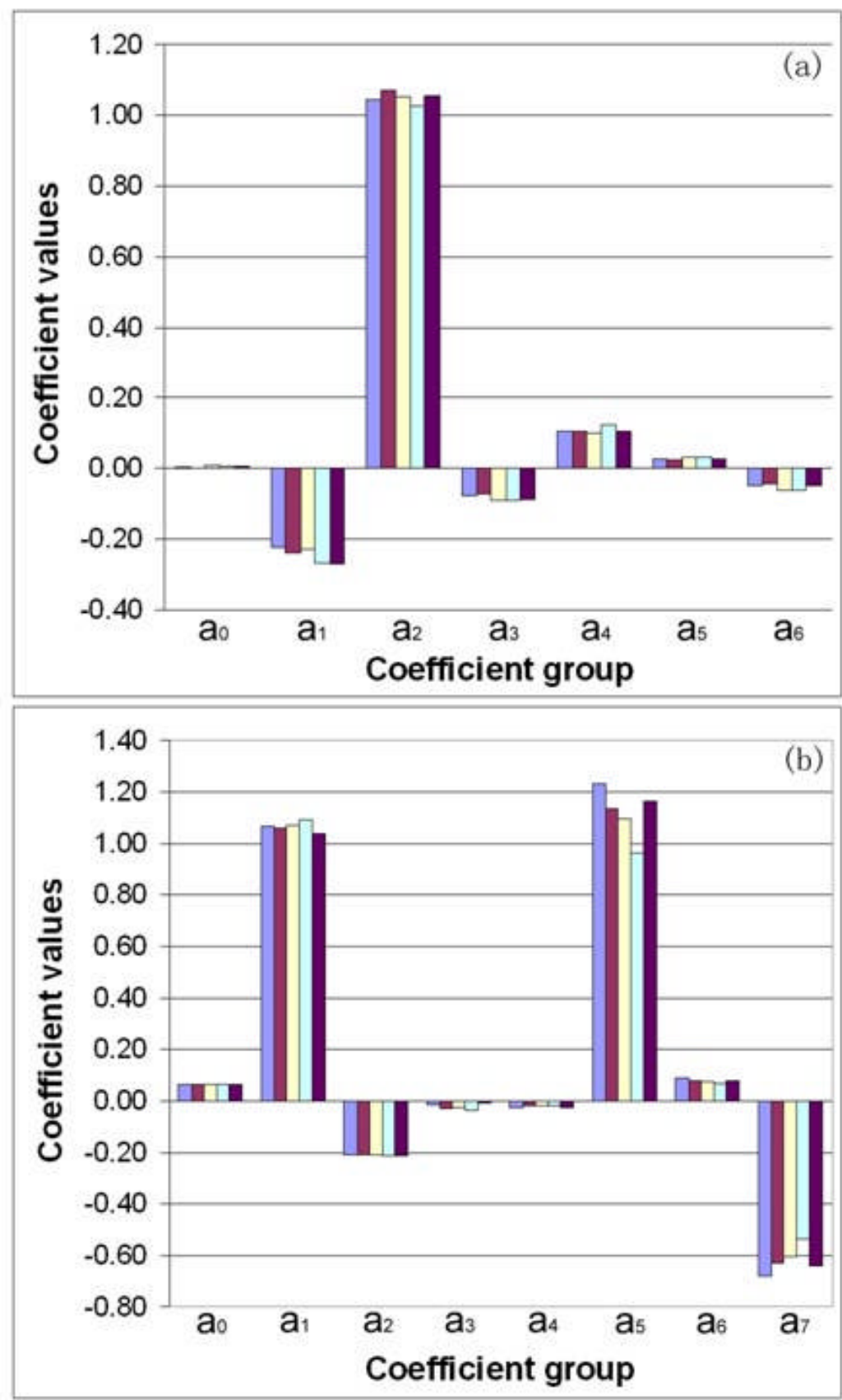

Figure 7. Regression model coefficients $(\mathrm{a} 0, \mathrm{a} 1, \ldots)$ retrieved in five runs with independent random sampling. Regression model is for MODIS-equivalent channel $B 1$ BRDF isotropic parameter for grassland and 10 day composite interval of May, 11-20, 2001. a) VGT model, Eq. (19); b) AVHRR model, Eq. (20). 


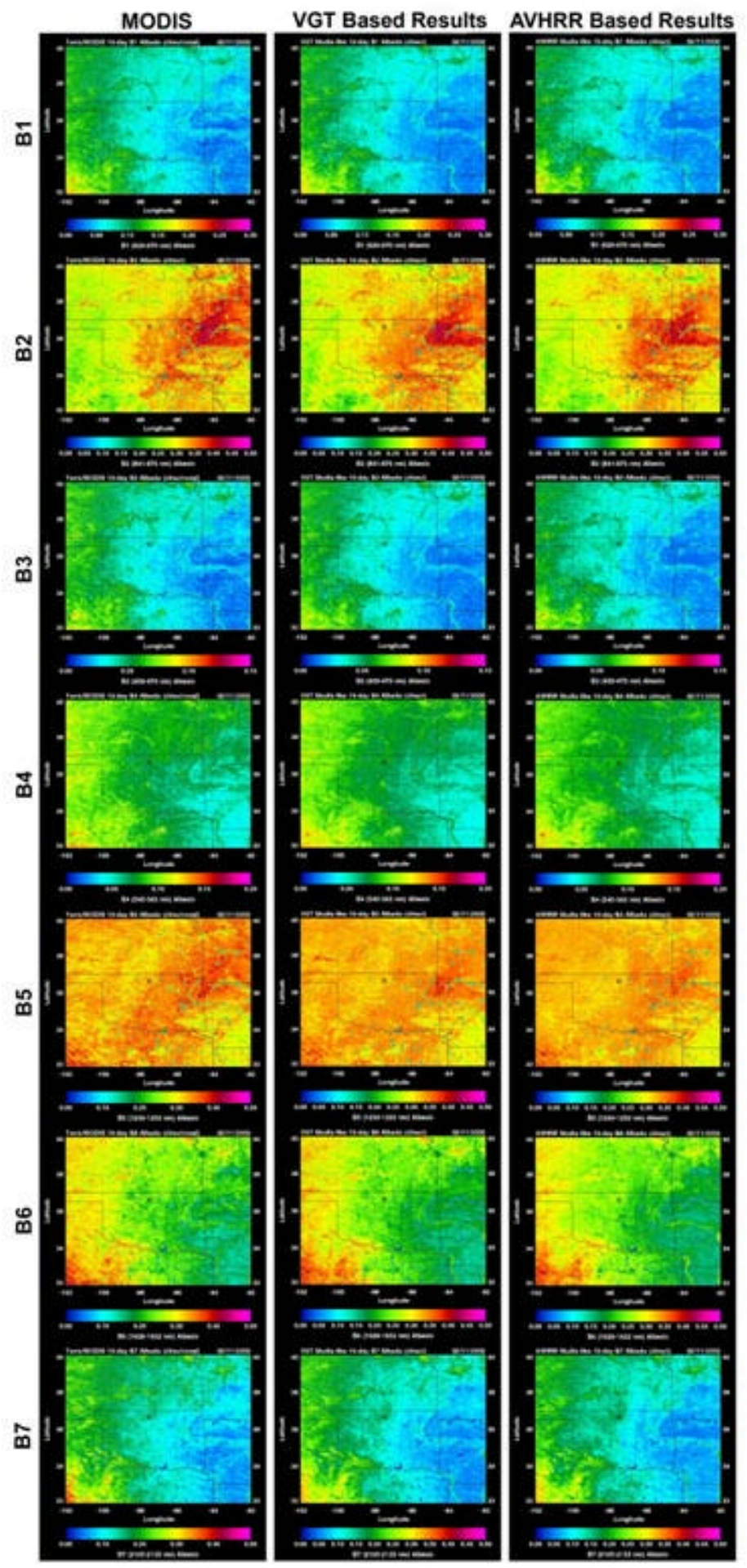

Figure 8. Surface albedo for seven spectral bands $B 1-B 7$ over the ARM SGP region derived from MODIS, VGT, and AVHRR observations for composite interval May 11-20, 2000. Direct albedo at local solar noon is shown. 

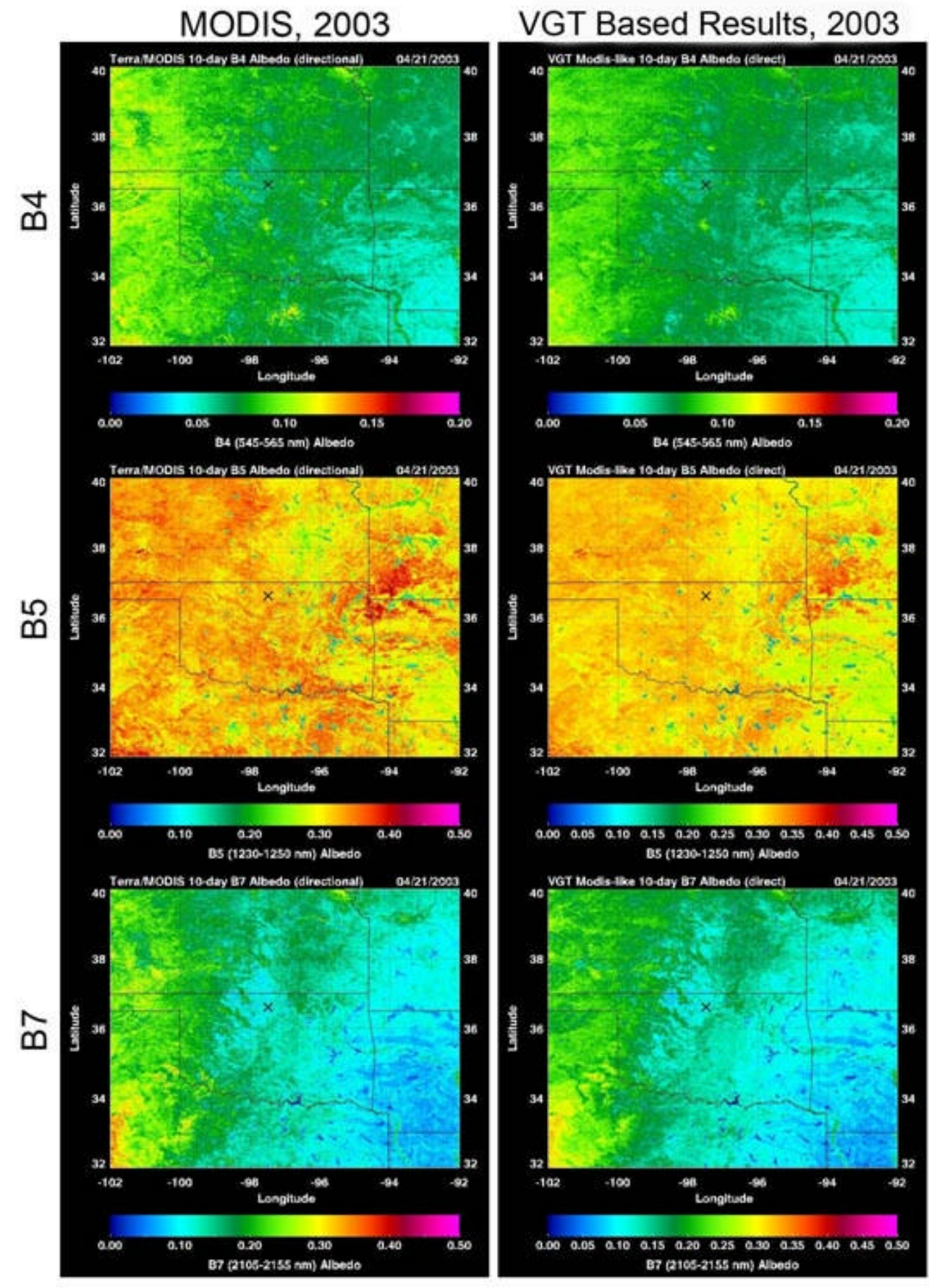

Figure 9. Assessment of surface albedo over the ARM SGP region observed from

MODIS and VGT-based replicates for independent composite period April 21-30, 2003.

Spectral channels $B 4, B 5$ and $B 7$ are shown. 

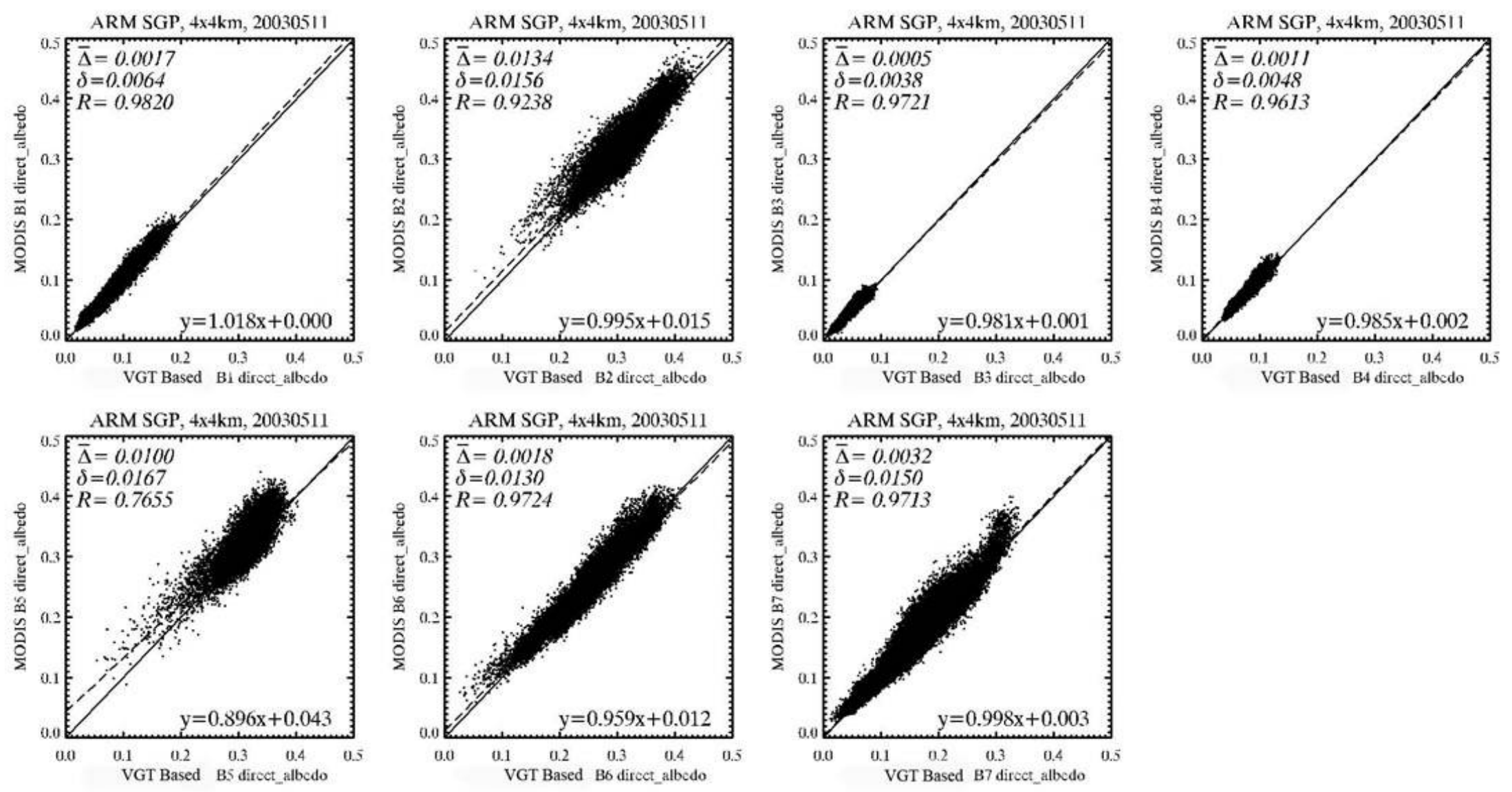

Figure 10. a) Scatter plots for the MODIS versus the VGT-based surface albedo for seven bands for composite interval May 11-20,

2003. b) the same as a) but for the AVHRR-based versus the VGT-based albedo for composite interval July 21-30, 1998. The mean bias between the two datasets $(\bar{\Delta})$, the standard deviation of the bias $(\delta)$, and the correlation coefficient $(R)$ are shown. 

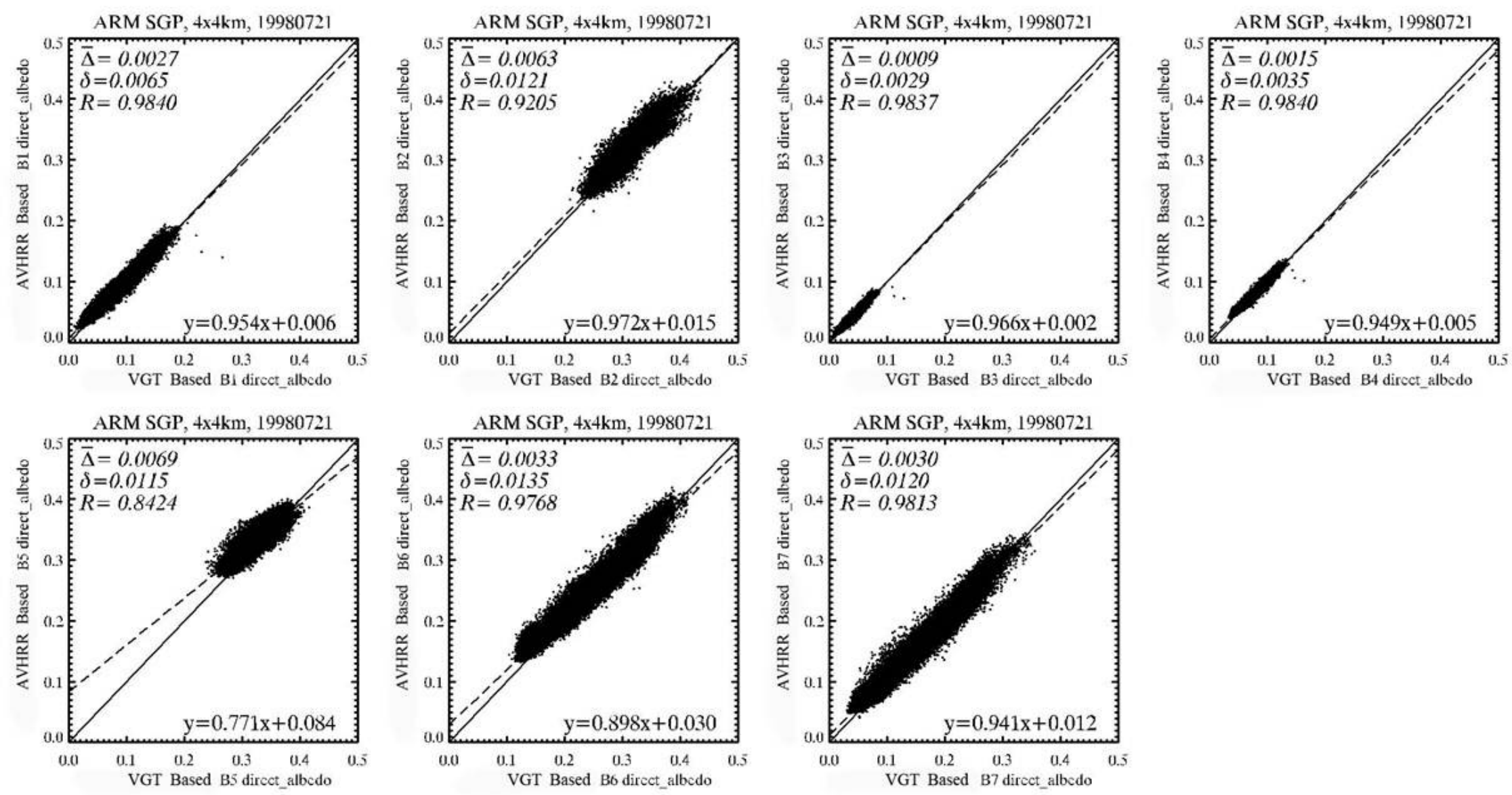

Figure 10b. same as 10a) but for the AVHRR-based versus the VGT-based albedo for composite interval July 21-30, 1998 

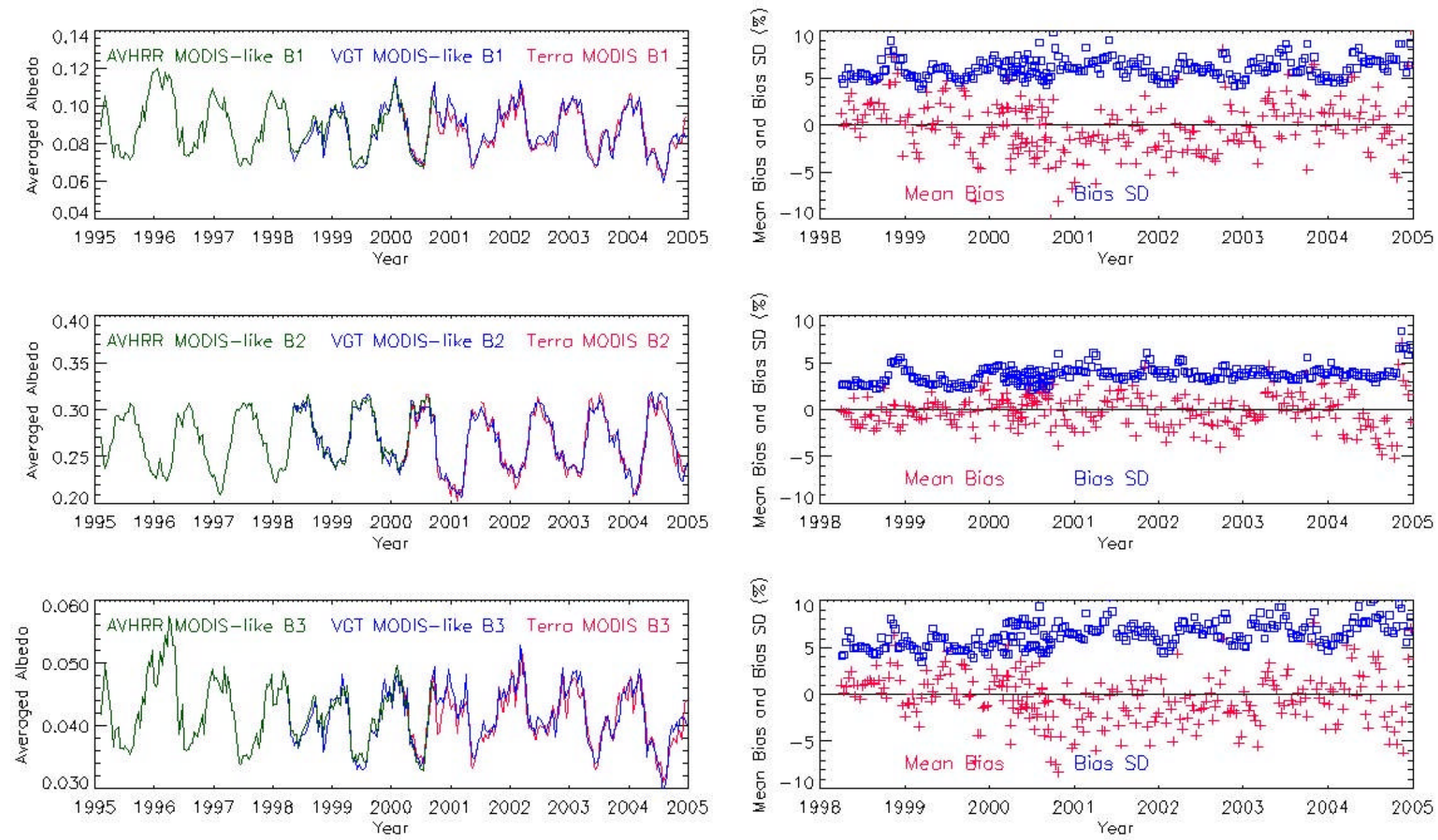

Figure 11a). Overall surface albedo statistics for the extended ARM SGP region derived from the AVHRR (1995-2000), VGT (1998-

2004), and MODIS/Terra (2000-2004) data at 10-day intervals. Left panels show mean albedo, right panels show relative mean bias (cross symbols) and standard deviation of bias (square symbols). a) bands $B 1-B 3$. b) bands $B 4-B 7$. 

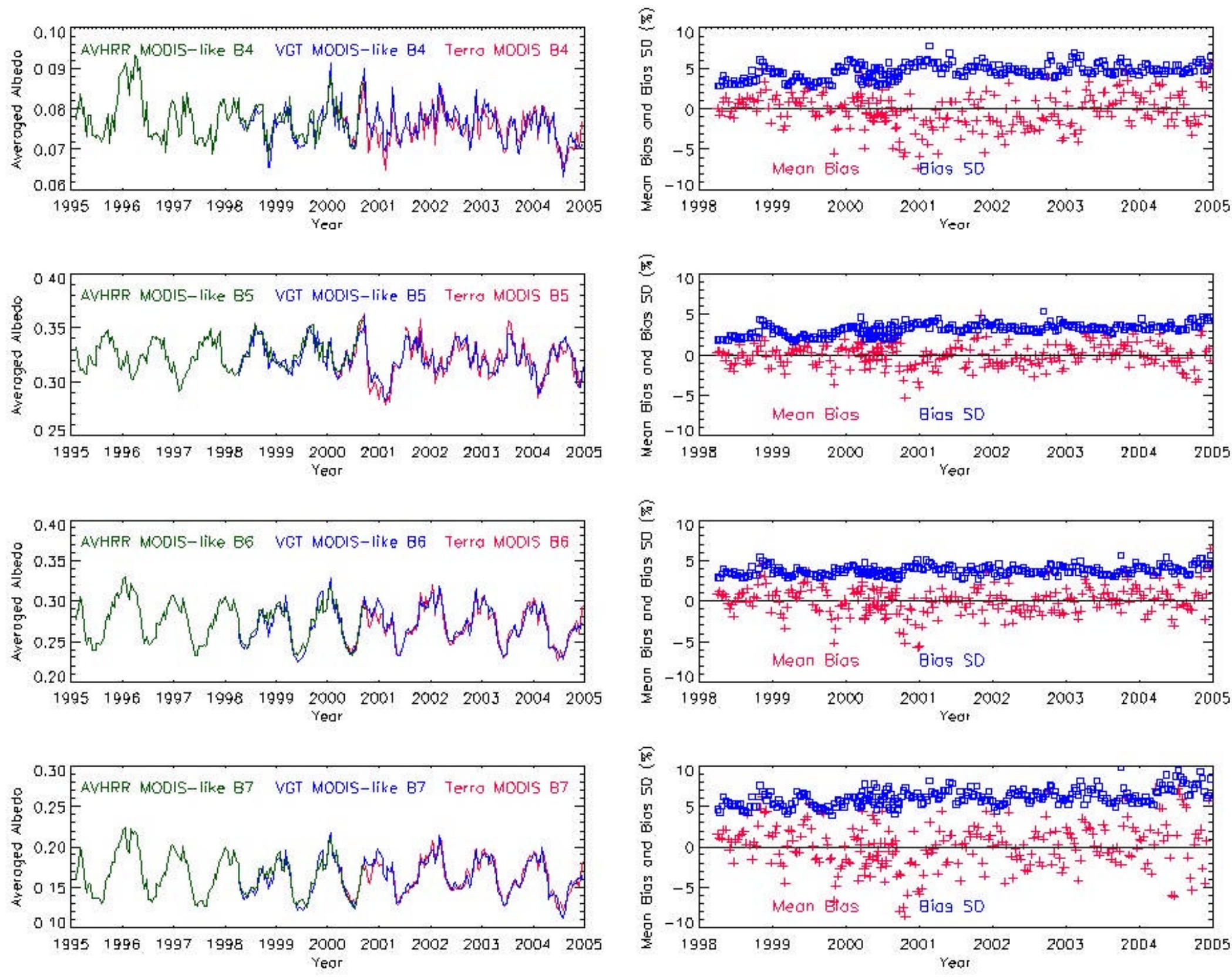

Figure 11b) the same as Figure 11 a) but for bands $B 4-B 7$. 


\title{
Effects of spectral response function on surface reflectance and NDVI measured with moderate resolution satellite sensors
}

\author{
Alexander P. Trishchenko ${ }^{(1)}{ }$, Josef Cihlar ${ }^{(1)}$, Zhanqing $\mathrm{Li}^{(1,2)}$ \\ (1) Canada Centre for Remote Sensing \\ Natural Resources Canada, Ottawa, Ontario, K1A 0Y7 Canada \\ (2) University of Maryland, ESSIC, College Park, MD, 20742, USA \\ * Corresponding author: \\ 588 Booth Street, Ottawa, Ontario, K1A 0Y7, Canada \\ Phone: (613) 9955787 \\ Fax: (613) 9471406 \\ e-mail: trichtch@ccrs.nrcan.gc.ca
}

Remote Sensing of Environment 
Abstract

We report the results of a modeling study on the sensitivity of Normalized Difference Vegetation Index (NDVI) and surface reflectance to differences in instrument spectral response functions (SRF) for various AVHRR radiometers onboard NOAA satellites NOAA-6 - NOAA-16 as well as the Moderate Resolution Imaging Spectroradiometer (MODIS), the VEGETATION sensor (VGT) and the Global Imager (GLI). Modeling results were validated against real satellite observations employing AVHRR/NOAA-14, -15 and MODIS, with a very good agreement. It is shown that for identical atmospheric state and similar surface spectral reflectance, the NDVI and spectral reflectances are sensitive to the sensor's SRF. Relative to a reference SRF for AVHRR/NOAA-9, the differences in reflectance among the AVHRR radiometers range from $-25 \%$ to $+12 \%$ for visible channel (red), and from $-2 \%$ to $+4 \%$ for NIR channel. Absolute change in NDVI among various AVHRRs ranged from -0.02 to +0.06 . The most significant difference was observed for the AVHRR/3 radiometer. Consistent results were obtained with the AVHRR sensors aboard the following afternoon satellites: NOAA-9, NOAA-11 and -12, whereas important discrepancies were found for other AVHRRs aboard NOAA-6, -10 and especially those launched more recently (NOAA-15, -16). Reflectance and NDVI measured by MODIS channels 1 and 2 also exhibit significant differences (up to 30-40\%) relative to AVHRR. GLI and VGT have some specific features that should be taken into account when intercomparing surface or top of the atmosphere reflectance as well as NDVI. Sensitivity of the SRF effect to variable atmospheric state (water vapor, aerosol, and ozone) was also investigated. Polynomial approximations are provided for bulk spectral correction with respect to AVHRR/NOAA-9. 
Keywords: satellite, surface reflectance, AVHRR, MODIS, GLI, VGT, NDVI, spectral response function, spectral correction, global change detection. 


\section{Introduction.}

Satellite observation is a convenient and feasible tool for global monitoring of atmospheric and terrestrial environment due to frequent and global coverage. Among various satellite sensors, the Advanced Very High Resolution Radiometer (AVHRR) onboard the National Oceanic and Atmospheric Administration's (NOAA) polar orbiting satellites has the longest record for research and application (Cracknell, 1997). There are three series of the AVHRR instruments. The four-channel radiometers AVHRR/1 were launched onboard Tiros-N, NOAA6, -8, -10. The five-channel radiometers AVHRR/2 were deployed on the platforms NOAA-7, 9, $-11,-12,-14$, followed by a six-channel radiometer AVHRR/3 onboard NOAA-15 and -16.

The range of AVHRR data applications is very broad. To name a few, e.g., the visible and NIR channels of AVHRR are used for retrieving cloud parameters (Rossow, 1989), solar radiation budget (Hucek and Jacobowitz, 1995), determination of absorbed photosynthetically active radiation (APAR) (Li et al, 1997), retrievals of aerosol optical depth (AOD) (Stowe et al, 1997) and other parameters (Nakajima et al., 2001; Gutman et al., 2000). One of the most important applications of the AVHRR thermal channels lies in estimation of global sea surface temperature (SST) (Reynolds and Smith, 1993). The thermal channels are also used for determining land surface temperature and emissivity (Qin and Karnieli, 1999). Thermal AVHRR channels in combination with channel 1 and 2 are employed for forest fire detection and monitoring (Li et al, 2000).

An important application of AVHRR solar channels is the retrieval of surface reflectance to determine different land surface parameters such as surface cover type (Cihlar et al., 2001), normalized difference vegetation index (NDVI) (Kidwell, 1994), Leaf Area Index (LAI) (Chen 
et al., 1997) and other surface characteristics. New opportunities for global monitoring of terrestrial ecosystem are unfolding with the availability of MODIS data.

The processing of satellite data involves many steps. The final purpose of satellite data processing in land surface studies is to obtain the systematic maps of various quantitative physical parameters corrected for the intervening effect of atmosphere, effect of varying observational geometry and specific sensor properties. Some of these corrections can be done quite accurately, like correction for Raleigh molecular scattering. Nonetheless, most of them may be implemented with some uncertainty due to limited knowledge of input information.

The important processing step is the data calibration. Despite numerous efforts, the results often vary among different investigators (Brest et al., 1997; Rao and Chen, 1999; Gutman, 1999; Masonis and Warren, 2001; Tahnk and Coakley, 2001). Accurate calibration requires continuos monitoring of the gain and offset due to degradation of sensor sensitivity with time. The degradation may not necessarily be a linear function of time (Tahnk and Coakley, 2001). It is commonly agreed that for satellite sensors lacking onboard calibration in solar spectrum, the total relative uncertainties of calibration are within 5\% (Rossow and Schiffer, 1999). An essential part of this uncertainty is related to the effect of spectral response function, when it is not accounted for properly during vicarious calibration or sensor intercalibration (Teillet et al., 2001).

Variable Sun and observational geometry induces another source of systematic noise (Gutman et al., 1989; Li et al., 1996). This angular effect is a combination of anisotropic reflective properties of the atmosphere and land surface. The effect must be accounted for in long-term studies of satellite data to obtain unbiased results (Cihlar et al., 1998; Gutman, 1999). This is achieved by normalizing satellite image to common geometry using empirical anisotropic 
factors. They are derived either from sequence of satellite scenes collected over long period of time (Cihlar et al., 1998, Trishchenko et al., 2001) or from special directional observations, like those ones from POLDER or MISR instruments (Csiszar et al., 2001). Neglecting angular correction in the AVHRR data processing, for example, may introduce biases in composite coarse resolution long-term reflectance datasets of the order of 1-2\% depending on spectral band and surface type (Gutman, 1999). The effect becomes more significant (5-10\%) for solar zenith angles greater than $55^{\mathrm{O}}$.

Numerous vegetation indices have been developed to monitor the state of vegetation from spaceborne instruments (Bannari et al., 1995). They were constructed to diminish atmospheric contamination, mitigate the influence of soil spectral reflectance signatures, or emphasize certain features of vegetation conditions. The set of advanced vegetation indices optimised for upcoming sensors is discussed by Gobron et al. (2000). Nevertheless, NDVI remains the basic vegetation index most widely employed for global monitoring of vegetation. It is defined as the following ratio

$$
N D V I=\frac{\rho^{N I R}-\rho^{\text {red }}}{\rho^{N I R}+\rho^{\text {red }}},
$$

where $\rho^{\text {NIR }}$ and $\rho^{\text {red }}$ are reflectances for visible (red) and NIR spectral bands.

Attempts have been made to use the AVHRR data for long-term monitoring of land reflectances and vegetation indices (Gutman, 1999; Kaufman et al., 2000; Cihlar et al. 2001). These and other studies on long-term monitoring are motivated by the availability of quality AVHRR time series for the period of nearly twenty years. Although the construction and characteristics of all AVHRR instruments are quite similar, they are not identical among all missions. Consequently, the effect of varying spectral response may create an artificial noise imposed upon a subtle natural variability. This artifact should be examined thoroughly before 
comparing data between different missions to determine possible changes in satellite climatic records. So far, the effects of spectral response functions have not been considered carefully in such studies. Some influence of the spectral characteristic of the satellite sensors on remote sensing of vegetation indices has been studied for forested regions (Teillet et al., 1997) and during vicarious calibrations procedures (Teillet et al., 2001). Nevertheless, systematic characterisation of these effects for various representative surface spectral signatures on a global scale and for all AVHRR sensors has not been addressed properly. Analysis of long-term satellite products from various missions may require corrections to account for differences in SRF that have not been investigated.

Our study is aimed to fill this gap and to provide quantitative estimates for the effect of SRF among all AVHHR missions. Differences between AVHRR and MODIS, GLI and VEGETATION sensors are also considered. To achieve this goal, some representative surface spectral reflectance curves were selected from two observation sources. The first is a database of spectral observation made by the PROBE-1 instrument (Secker et al., 1999) at the Canada Centre for Remote Sensing (CCRS). The second one is the ASTER (Advanced Spaceborne Thermal Emission and Reflection Radiometer) spectral library (available from http://speclib.jpl.nasa.gov). The details of the databases are given below. The 6S radiative transfer code (Vermote et al., 1997) was employed for model simulation of the signal at the top-of-the-atmosphere (TOA) level under various atmospheric conditions and observational geometries.

The paper is organized as follows. The next section describes the special features of instrument spectral response functions. Section 3 discusses the surface spectral library and modeling of satellite signal at the TOA level. Section 4 presents results and an analysis of 
comparisons between various sensors. Validation results of the model simulation using real satellite observations are shown in Section 5. Section 6 summarizes the research.

\section{Sensor spectral response functions}

The SRFs for AVHRR NOAA-6 to -16, MODIS, GLI and VGT compatible channels in visible and NIR are shown in Figure 1a-c. The three panels of Figure 1 present spectral response functions for different types of AVHRR: AVHRR/1(a), installed on morning satellites, AVHRR/2(b), which was operational on afternoon satellites, and a morning satellite NOAA-12. The bottom panel c) shows spectral response functions for AVHRR/3 (NOAA-15 and 16), MODIS, GLI and VGT. A typical spectrum of green vegetation is also plotted for reference. Though similar, these curves differ in shape, the central wavelength location, the bandwidth and the degree of overlap between channels, especially with respect to the transition from chlorophyll absorption band to foliage reflection band $(0.68-0.72 \mu \mathrm{m})$.

Noticeable differences are seen among various AVHRR radiometers. The most notable differences exist between AVHRR/3 on NOAA-15, 16 and other AVHRR radiometers (Figure 1b-c). The channels of the new AVHRR/3 radiometers have narrower bandwidths and a much smaller overlap over the vegetation transition band. All these factors affect the magnitude of spectral reflectance observed by the sensors and lead to higher NDVI values over vegetated surfaces.

The MODIS channels are also quite distinct from the AVHRR ones (Figure 1c). They are much narrower and have no overlap with each other over the vegetation transition band. The 
MODIS channel 1 is also shifted further to near IR region relative to the AVHRR channel 1 . The GLI red channel is close to MODIS, although it is shifted more toward the NIR. The width of the GLI NIR channel is between those for AVHRR and MODIS. The VGT red channel extends beyond $0.7 \mu \mathrm{m}$ limit, thus causing significant impact on the red reflectances and NDVI. Figure 1 indicates that a direct comparison of spectral reflectance or vegetation indices produced by various sensors should be made with caution.

\section{Surface spectral data and modeling}

To encompass a potential range of variability in surface reflectance and NDVI, a set of representative spectra for various surface targets were compiled, following the classification scheme used in the NASA Surface and Atmospheric Radiation Budget (SARB) Project (Rutan and Charlock, 1997). The complete scheme for the SARB Project included 20 different surface

classes. Since we had no measurements for some of the surface types and yet the particular focus of this study is on the boreal ecosystem, 12 classes were adopted in this investigation (Figure 2 and Table 1).

Surface classes of lower class number nevertheless covered the bulk of variability in spectral reflectance occurred in nature. Classes 1 and 2 are depicted by two spectral curves each, and class 6 (grassland) is described by 4 curves with different values of NDVI to reflect various vegetation density levels.

Spectral curves were derived from two sources: aircraft observations from the PROBE-1 instrument made by the Canada Centre for Remote Sensing (Secker et al., 1999). The PROBE-1 
is airborne hyper-spectral sensor covering visible and near-infrared spectral regions with 128 spectral bands. The second source was acquired from the ASTER spectral library. We normalized each spectrum to reproduce SARB broadband albedo, i.e.

$$
\tilde{\rho}(\lambda)=\alpha_{\text {SARB }} \frac{\int_{\lambda \min }^{\lambda \max } S_{0}(\lambda) d \lambda}{\int_{\lambda \min }^{\max } S_{0}(\lambda) \rho(\lambda) d \lambda},
$$

where $S_{0}(\lambda), \rho(\lambda)$ are the solar spectral constant and observed spectral reflectance, and $\alpha_{\text {SARB }}$ is the broadband albedo from Rutan and Charlock (1997). The normalization (1) provides a link of individual spectral curves to a specific SARB surface type. The derived spectra (Figure 2) are shown for the wavelength interval 0.35-1.25 $\mu \mathrm{m}$, which essentially covers visible and NIR portion of the solar spectrum under study. The top panel (Figure 2a) depicts spectra of nonvegetated surfaces and a dry grass/savannah surface, and the bottom panel (Figure $2 \mathrm{~b}$ ) shows spectra for various vegetated surfaces. The steep increase in reflectivity above $0.7 \mu \mathrm{m}$ is a characteristic feature of these curves. The data selected encompass spectral differences between various natural surface classes.

\section{Modeling results}

\subsection{Radiative transfer modeling.}

The 6S radiative transfer model was employed to simulate the TOA signal. The wavelength increment of the model is $2.5 \mathrm{~nm}$, which allows us to accurately resolve all spectral features of the targets and instrument SRF. Baseline simulations were conducted for US62 
atmospheric profile with total water vapor (TWV) columnar amount scaled to $1.5 \mathrm{~cm}$, representative for the boreal region in summer time (Cihlar et al., 2000). The ozone content was set to 350 Dobson units (DU). Since satellite studies of surface properties usually employ clearsky composites selected for the highest atmospheric transparency, the AOD was set to 0.06 , as recommended by Fedosejevs et al. (2000) for the Canadian boreal zone. The surface reflectance was assumed to be Lambertian, i.e. independent of sun-sensor geometry. The TOA reflectance has a certain dependence on observational geometry due to atmospheric effects. We conducted computations for various geometrical conditions: the solar zenith angle (SZA) $\theta_{0}$ varying from $0^{0}$ to $75^{0}$ with $15^{0}$ steps, the viewing zenith angle (VZA) $\theta$ from $0^{0}$ to $45^{\circ}$ with $15^{0}$ steps, and the relative azimuth angle (RAA) $\phi$ was set to $0^{0}, 90^{\circ}$ and $180^{\circ}$. For sensitivity tests, the following values were chosen: 0, 0.06 and 0.6 for AOD; $0.5,1.5$ and $5.0 \mathrm{~cm}$ for TWV; $270 \mathrm{DU}, 350 \mathrm{DU}$ and $430 \mathrm{DU}$ for ozone. The simulations were done for a viewing geometry at $\theta_{0}=45^{\circ}, \theta=0^{0}$, $\phi=0^{0}$, a typical geometry for normalizing satellite observations (Trishchenko et al., 2001).

The range of variability in reflectance and NDVI values simulated for the AVHRR radiometer on board NOAA-9 over all surface targets is illustrated in Figure 3. This radiometer is often considered as a reference instrument (e.g., Rossow and Schiffer (1999)). Note the break in vertical scale for visible channel in Figure 3a, which was introduced to show enough details for low reflective surfaces as well as the highly reflective snow surface. The reflectance in visible (red) band for vegetated surfaces ranges from 0.05 to 0.15 . At the surface, the values are typically smaller because scattering by atmospheric molecules adds to the signal reflected from darker surfaces. The opposite is true for the NIR band where surface reflectance is higher than at the TOA, because atmospheric attenuation outweighs scattering back to the sensor. These relationships also explain why NDVI at the TOA level is typically smaller than at the surface 
level. The NDVI is either very small or even negative for non-vegetated surfaces, such as water, snow and barren/desert classes.

\subsection{Surface level}

Figures 4-6 show the surface level results for visible (red), NIR reflectances and NDVI. The absolute and relative differences with respect to AVHRR/NOAA-9 values are plotted against NDVI for each sensor. The difference is also referred to as the spectral correction factor. The least differences are found for AVHRR/NOAA-11 followed by NOAA-12. Other AVHRR/1,2 radiometers are reasonably close to AVHRR/NOAA-9, although the differences could reach 0.01 (10-15\% relative) for red channel (Figure 4), 0.01 (2-3\%) for NIR channel (Figure 5), and 0.03 (4-6\%) for NDVI of vegetated surfaces (Figure 6). Since NDVI for sparse vegetation and non-vegetated targets are small, the relative differences in NDVI for these surface types are larger.

The sensor spectral reflectances and NDVI differ systematically for AVHRR/3 onboard NOAA-15 and NOAA-16. The visible (red) reflectance for AVHRR/3 is smaller by 0.01-0.015 (20-25\%) (Figure 4), while the NIR channel reflectance is larger by 0.01-0.015 (3-4\%) (Figure 5). As a result, NDVI derived from AVHRR/NOAA-15 or -16 is higher by $0.03-0.06$ (5-10\%) (Figure 6). These differences are due to (i) a significantly narrower spectral band of the visible (red) channel that is much less contaminated by the elevated reflection in NIR; (ii) the NIR channel is less influenced by the transition band (Figure 1c).

The SRFs of MODIS and GLI are so different from that of AVHRR/NOAA-9 that the surface reflectance differences reaches 0.02 (20-30\%) in visible channel (Figure 4), 0.04-0.05 (10-15\%) in NIR channel (Figure 5), and 0.06-0.09 (20-25\%) in NDVI (Figure 6). The SRF 
effect for VGT is smaller than for MODIS and GLI and comparable to AVHRR/3. The difference could be as large as $0.01(\sim 10 \%)$ in the visible (red) channel, $0.02-0.04$ (5-10\%) in the NIR channel, and 0.03 ( $5-10 \%)$ in NDVI for vegetated surfaces.

The discrepancies caused by different SFRs may be corrected using the second degree polynomial functions, as shown in Figures 4-6. The curves were produced by fitting the data points. Tables 2, 3 and 4 give the coefficients of the quadratic functions that best fit the data, correlation coefficient and standard deviation of the fit for each sensor for visible, NIR and NDVI respectively. The quality of the fits is quite good for AVHRRs and MODIS, while data for VGT and GLI sensors are more scattered.

\subsection{TOA level}

The general trends of the effect of SRF at the TOA are similar to those at the surface, but they do differ in detailed features as a result of the distortion by the atmosphere. Discrepancies in TOA visible, NIR reflectance and NDVI with respect to AVHRR/NOAA-9 are shown in Figure 7-9. Quadratic fits to the relative differences in reflectances and absolute difference in NDVI are also plotted. Other data points are more scattered and no fits are presented. For example, the relative difference for NDVI may be extremely large for some combinations, simply because NDVI values computed at the TOA level are very close to zero. Figure 7-9 contains more points than figures for the surface level because of the variable effects of the atmosphere and observation geometry. Therefore, fitting all data points with one curve is just a bulk approximation of the SRF effect to account for the large SRF effect (e.g., MODIS, VGT, GLI). In the case of AVHRR sensors, the approach still provides a good approximation of the effect of SRF. The parameters of fitting curves are given in Tables 5-7. 
Similar to the surface case, the best agreement with AVHRR/NOAA-9 was found for AVHRR/NOAA11. For all remaining AVHRRs, the atmospheric effect generally diminishes the spectral difference for the visible (red) channel and slightly increases it in the NIR. The absolute discrepancies in NDVI remain essentially the same as at the surface, 0.03-0.06 (Figure 9). The effect on NDVI for NOAA-7, $-8,-11,-12$ and -14 is typically within \pm 0.01 . For NOAA-6 and NOAA-10, the differences in NDVI relative to NOAA-9 were as much as $0.02-0.03$ (3-5\% for vegetated surfaces). The largest discrepancy was observed for NOAA-15 and -16 . The absolute difference in NDVI could be as high as $0.03-0.06$, which is larger than $10 \%$ for vegetated targets.

The corrections for visible channels of MODIS, VGT and GLI sensors at the TOA level shown in Figure 7 are similar in magnitude to those at the surface, but the magnitude of spectral correction for the NIR channels is much higher (Figure 8). Apart from the same reasons as for surface, the narrow NIR spectral channels are not affected by atmospheric absorption in the 0.94 $\mu \mathrm{m}$ water vapor absorption band. Consequently, the relative differences between these sensors and AVHRR/NOAA-9 were as much as $20-40 \%$, with the largest differences occurred between AVHRR and MODIS (up to 40\%). The results for VGT and GLI are similar but smaller than for MODIS. The difference ( $\rho_{\text {NIR }}-\rho_{\text {NIR,NOAA-9 }}$ ) is positive in all cases and increases with NDVI.

\subsection{Sensitivity of spectral correction to atmospheric state}

Although the spectral effects discussed above are representative for 'nominal' conditions described above, it is also likely that in any particular comparison the magnitude of the differences is affected by the variable atmospheric state. Major variables influencing satellite 
measurements are aerosol optical depth (AOD), total water vapor (TWV) and ozone columnar amounts. To investigate how variations in the atmospheric state affect the spectral correction factors, a sensitivity factor of spectral correction $(\Delta \rho)^{\prime}$ to the atmospheric constituent $A$ is defined as

$$
(\Delta \rho)_{i}{ }^{\prime}=\Delta \rho_{i}(A)-\Delta \rho_{i}\left(A_{0}\right),
$$

where

$$
\Delta \rho_{i}(A)=\rho_{i}(A)-\rho_{\text {AVHRR / NOAA-9 }}(A),
$$

$A$ is the amount of TWV, ozone or AOD; $A_{0}$ is the corresponding amount for the basic (or reference) atmospheric state, index $i$ refers to a specific satellite sensor, and $\rho$ denotes the reflectance or NDVI. Normalized (relative) sensitivity is computed as the ratio $\left[(\Delta \rho)^{\prime} / \rho_{\text {AVHRR / NOAA-9 }}\right] * 100 \%$.

Water vapor amount. The sensitivity tests of spectral effect to the amount of TWV in the atmospheric column are computed for the NIR channels and NDVI (Figs. not included), since water vapor effect in the visible band is not significant. The tests were conducted for the basic atmospheric state $(1.5 \mathrm{~cm})$ and two extreme values, $0.5 \mathrm{~cm}$ (dry case) and $5 \mathrm{~cm}$ (humid case); the ozone amount was fixed at $350 \mathrm{DU}$ and the aerosol optical depth at 0.06 . The sensitivity of spectral correction to precipitable water vapor is rather small for the AVHRR NIR channels relative to the magnitude of the spectral correction itself. It is well within $1 \%$ of AVHRR/NOAA-9 reflectance and typically 3-5 times smaller than spectral correction computed for the basic atmospheric state. Since water vapor has essentially no effect on visible reflectance, corrections to NDVI are also small. For other sensors (MODIS, VGT, GLI), the sensitivity to water vapor is somewhat larger. The spectral adjustment for MODIS NIR channel 
is about $-6 \%$ and $7-8 \%$ for $\mathrm{TWV}=0.5 \mathrm{~cm}$ and $5 \mathrm{~cm}$ respectively, relative to the standard case of TWV=1.5 cm. Corrections for NIR channel reflectance of VGT and GLI sensors are within $\pm 3 \%$. For NDVI, absolute corrections range from -0.03 to +0.04 for MODIS and from -0.015 to 0.02 for NDVI of VGT and GLI.

Based on these results, we may conclude that the effect of atmospheric water vapor is negligible when making spectral adjustments among various AVHRR radiometers. This is because the various sensors have similar spectral coverage, in particular with respect to water vapor absorption bands. Note that the spectral correction effect under study should not be confused with the absolute effect of TWV on NIR reflectance itself. The latter is a lot more significant (Cihlar et al., 2000). The situation is more complicated for MODIS, VGT and GLI sensors. The TOA reflectances measured by these sensors are less sensitive to water vapor, since SRF for these instruments do not include the strong water absorption band around $0.94 \mu \mathrm{m}$. Nevertheless, since we estimate the effect of spectral correction relative to AVHRR, which is quite sensitive to water vapor amount (Cihlar et al., 2000), the sensitivity of spectral correction to water vapor emerges for these sensors.

Aerosol optical depth. The sensitivity of the spectral effects to aerosol optical depth was examined assuming a continental aerosol with $\mathrm{AOD}=0,0.06$ and 0.6 ; $\mathrm{TWV}=1.5 \mathrm{~cm}$, ozone amount $=350$ DU. Since the effect is very small $(<0.2 \%)$ for low aerosol loading, we focus on the effect caused by moderately large aerosol amounts. The sensitivity of the spectral correction to aerosol for the red channel of AVHRR (relative to AVHRR/NOAA-9) is negligible for AVHRR/NOAA-8, 11 and 12 (Figure 10). It may reach 2-3\% for AVHRR/NOAA-6, 7 and 10, and exceed 3\% for NOAA-15 and -16. The correction is negative for AVHRR/NOAA-14 (-1 - 
$2 \%)$. The absolute magnitude of the correction ranges from -0.001 to +0.002 . The sensitivity is similar for other sensors (Figure 10).

The sensitivity for NIR channels of AVHRR is even smaller in general (Figs. omitted) (-0.3\% -1\%), and somewhat larger for MODIS, VGT and GLI (-2\% to $-5 \%)$ NIR channels. The magnitude of sensitivity of the NDVI spectral correction to AOD falls within $-3 \%$ to $+3 \%$. While such an effect is comparable with the instrument uncertainties, the effect is persistent for aerosol laden atmosphere, and is thus recommended to be taken into consideration in intercomparison studies, if possible.

Ozone amount. The sensitivity of the spectral correction to ozone amount is studied for 270 DU, 350 DU and 430 DU. Since ozone has a weak absorption in the visible region centered around $0.6 \mu \mathrm{m}$ (Chappius band) (Liou, 1992), the sensitivity is overly small. For all AVHRR radiometers and MODIS, the correction is typically less than $\sim 0.5 \%$ or 0.001 in absolute units. For VGT and GLI, the effect is slightly larger, $0.5 \%-1 \%$ relative or 0.002 absolute. The magnitude of the ozone effect on the NDVI spectral correction is equally small, as it essentially affected by changes in visible reflectances only.

\section{Application to real satellite data}

To test the modeling results, we compared two pairs of images over identical areas. One pair is for AVHRR images acquired by NOAA-14 and NOAA-15, and another pair is for AVHRR/NOAA-14 and MODIS. The images in each pair were taken very close in time, so that 
temporal changes do not affect the comparisons. Two AVHRR images were taken over an area of Northern Ontario (Canada) observed on July 15, 2000 in the morning (NOAA-15) and afternoon (NOAA-14). The area is approximately $250 \mathrm{~km} \times 250 \mathrm{~km}$ centered around $\left(53^{\mathrm{O}} \mathrm{N} ; 87.5^{\mathrm{O}} \mathrm{W}\right)$.

The second comparison is between clear-sky composites from MODIS and AVHRR/NOAA-14 over an area of Southern Great Plains (USA). MODIS covers the period 1926 July, 2000 and the AVHRR for the period 21-31 July, 2000. All images were resampled to Lambert conformal conic projection with $1 \mathrm{~km}$ resolution. The area of comparison is $10^{\mathrm{O}} \mathrm{x} 8^{\mathrm{O}}$ centred around $\left(36^{\circ} \mathrm{N} ; 97^{\circ} \mathrm{W}\right)$. Joint statistical distributions of reflectances and brightness temperatures were analysed and additional thresholds were applied to all images to detect and to remove cloud-contaminated pixels in addition to clear-sky compositing procedures. Water pixels were excluded from the comparison due to strong directional effects that would complicate the comparison (Cihlar et al., 2001). The directional effects resulting from different local observation times and geometry do exist over land as well (Li et al., 1996) but less pronounced than water bodied. The effect is further reduced for NDVI due to considerable cancellation of the effects in the visible and NIR channels (Gutman, 1999, Li et al. 1996).

The comparisons of NDVI between AVHRR/NOAA-14 and 15 and between AVHRR/NOAA-14 and MODIS are presented in Figures 11-12. Statistical analyses using the ttest showed that the two comparisons have statistically significant non-zero mean difference at a significance level 0.01 or lower. The modal value of the distribution of NDVI differences between AVHRR/NOAA-15 and AVHRR/NOAA-14 shown in Figure 11 is 0.025 or 5.7\%. The difference between average values is slightly smaller ( 0.017 or $3.9 \%)$ because of skewness of the distribution. This difference is in conformity with modeling results, which also indicate a greater 
magnitude of NDVI for AVHRR/NOAA-15 than for AVHRR/NOAA-14. The magnitude of differences between modeled NDVIs is slightly larger (1.4\%) due to contributions by various factors, in particular due to aerosol and surface directional effects.

The directional effect is lessened for the comparison between MODIS and AVHRR/NOAA-14 due to closer observation times (Figure 12). The observed modal value of the NDVI difference is 0.14 (29\%) and the mean difference of 0.15 (31\%), in comparison with the modeled NDVI difference of about 0.125 (25\%). Slightly smaller values of NDVI difference for the modeling case may reflect the contribution of water vapor effect, as discussed in Section 4. Good overall agreement between modeling estimates and satellite observations bolsters our confidence in the estimates of spectral correction effects derived in this paper.

\section{Conclusions}

Long-term monitoring of the Earth's environment by satellite sensors require consistent and comparable measurements. In this paper, we evaluated the effect of a major sensor parameter, namely, the spectral response function, on the consistency of observations made by moderate resolution sensors commonly used for surface and atmospheric studies. Starting with TIROS-N in 1978, these sensors have provided a long time series of satellite data, which contain rich information pertaining to the state and changes of many important environmental and meteorological variables. However, use of such diverse data sets requires a careful evaluation of their compatibility and consistency to avoid any artifact. This study elaborates the influence of different SRF on reflectance measurements in the visible and NIR channels and on their 
combination in the form of NDVI. The sensors under study include AVHRRs from NOAA-6 to the latest NOAA-16 as well as MODIS, VGT and GLI. All the sensors are compared to the AVHRR/NOAA-9 radiometer, which was chosen as a reference.

The study illustrated that the differences in SRF are significant enough to be taken into account, in particular for studies concerning inter-annual variations. It is comparable in magnitude to the uncertainties caused by sensor calibration and the angular correction procedure. Even among "the same type" instruments such as AVHRR, the effect of the varying spectral response function on surface and TOA spectral reflectances and NDVI vegetation index is sufficiently large to require correction. Relative to the AVHRR/NOAA-9, differences range from $-25 \%$ to $+12 \%$ for visible reflectance (red), and from $-2 \%$ to $+4 \%$ for NIR reflectance. The absolute differences in NDVI among various AVHRRs range from -0.02 to +0.06 . The most consistent with AVHRR/NOAA-9 results were obtained for AVHRR/NOAA-11 and -12 where the corrections are small and optional. The corrections must be implemented for other AVHRRs and especially for the AVHRR/3 on NOAA-15 and -16. Reflectances and NDVI from MODIS differ from AVHRR/NOAA-9 by as much as 30-40\%. Likewise, VGT and GLI also exhibit considerable differences relative to AVHRR observations.

Given the significant effect of SRF, simple polynomial approximations were derived that may be used for correction. They provide a good accuracy of approximation for the AVHRR sensors. Other sensors (MODIS, VGT, GLI) require more significant correction to adjust for spectral differences in comparison with AVHRR. Polynomial approximations, we propose for these sensors, may be used as first-order corrections. Higher accuracy may be achieved by taking into consideration the atmospheric variables, observational angles, and information from additional spectral channels available from these instruments (Gitelson and Kaufman, 1998). 
Sensitivity tests of the SRF effect to various atmospheric variables (water vapor, aerosol, ozone) were conducted. In general, their influences are rather small. The largest effect is caused by aerosol, which may reach a few percent. Water vapour affects the spectral correction between AVHRR and other sensors, but not within AVHRR modifications. Ozone variation generally exerts a small effect for all sensors and may be neglected. The effects of SRF are further reinforced by analyses of two pairs of real satellite imagery data for AVHRR from NOAA-14 and -15 and from MODIS. The observational results are generally in good agreement with model simulations, both in sign and magnitude of the spectral response function effect.

\section{Acknowledgements}

Authors are grateful to J.-C. Deguise and R. Hitchcock of CCRS for making PROBE-1 data available for this study. We acknowledge the use of spectral data from JPL ASTER spectral library (http://speclib.jpl.nasa.gov). The authors also thank Gunar Fedosejevs for his valuable comments and discussion. This research was partially supported by the Biological and Environmental Research Program (BER), U.S. Department of Energy, Grant No. DE-FG0202ER63351. 


\section{References}

Bannari, A., Morin, D., and Bonn, F. (1995), A review of vegetation indices. Remote Sensing Reviews, 13, 95-120.

Brest, C. L., Rossow, W. B., and Roiter, M. D. (1997), Update of radiance calibrations for ISCCP. Journal of Atmospheric and Oceanic Technology, 14, 1091-1109.

Chen, J.M., Rich, P. M., Gower, T. S., Norman, J. M., Plummer, S. (1997), Leaf area index of boreal forests: theory, techniques and measurements. Journal of Geophysical Research, 102, 29 429-29 444.

Cihlar, J., Chen, J. M., Li, Z., Huang, F., Latifovic, R., Dixon, R. (1998), Can interannual land surface signal be discerned in composite AVHRR data? Journal of Geophysical Research, 103, 23163-23172.

Cihlar, J., Latifovic, R., Chen, J., Trishchenko, A., Du, Y., Fedosejevs, G., and Guindon, B. (2001), Systematic corrections of AVHRR image composites for temporal studies, Remote Sensing of Environment (in press).

Cihlar, J., Tcherednichenko, I., Latifovic, R., Li, Z., and Chen, J. (2000), Impact of variable atmospheric water vapor content on AVHRR data corrections over land. IEEE Transactions on Geoscience and Remote Sensing, 39 (1), 173-180.

Csiszar, I., Gutman G., Romanov P., Leroy M., Hautecoeur O. (2001), Using ADEOS/POLDER data to reduce angular variability of NOAA/AVHRR reflectances. Remote Sensing of Environment, 76, 399-409.

Cracknell, A. P. (1997), The advanced very high resolution radiometer (AVHRR). Taylor \& Francis, London, 534 pp. 
Fedosejevs, G., O'Neill N. , Royer A. , Teillet P. M. , Bokoye A. I. , McArthur B. (2000), Aerosol optical depth for atmospheric correction of AVHRR composite data; Canadian Journal of Remote Sensing, 26, 273-284

Gitelson, A., Kaufman, Y. J. (1998), MODIS NDVI optimization to fit the AVHRR data series spectral consideration. Remote Sensing of Environment, 66, 343-350.

Gobron, N., Pinty, B., Verstraete M. M., and Widlowski, J-L. (2000), Advanced vegetation indices optimized for up-coming sensors: Design, performance, and applications. IEEE Transactions on Geoscience and Remote Sensing, 38(6), 2489-2505.

Gutman, G., Gruber, A., Tarpley, D., and Taylor, R. (1989), Application of angular models to AVHRR data for determination of the clear-sky planetary albedo over land surfaces. Journal of Geophysical Research, 94, 9959-9970.

Gutman, G. (1999), On the use of long-term global data of land reflectances and vegetation indices derived from the advanced very high resolution radiometer. Jornal of Geophysical Research, 104(6), 6241-6255.

Gutman, G., Csiszar I., Romanov P. (2000), Using NOAA/AVHRR products to monitor El Niño impacts: Focus on Indonesia in 1997-98. Bulletin of the American Meteorological Society, 81, 1189-1205.

Hucek, R., Jacobowitz, R. (1995), Impact of scene dependence on AVHRR albedo models. Journal of Atmospheric and Oceanic Technology, 12( 4), 697-711.

Kaufman, R.K., Zhou, L., Knyazikhin, Y., Shabanov N. V., Myneni, R. B., and Tucker C. J. (2000), Effect of orbital drift and sensor changes on the time series of AVHRR vegetation index data. IEEE Transactions on Geoscience and Remote Sensing, 38(6), 2584-2597. 
Kidwell, K. (1994), Global Vegetation Index User's Guide, U.S. Dept. of Commerce, NOAA/National Environmental Satellite Data and Information Service, National Climatic Data Center, Satellite Data Services Division.

Li, Z., Cihlar, J., Zheng, X., Moreau, L., and Ly, H. (1996), The bidirectional effects of AVHRR measurements over boreal regions. IEEE Transactions on Geoscience and Remote Sensing, 34, 1308-1322.

Li, Z., Moreau, L., and Cihlar, J. (1997), Estimation of photosynthetically active radiation absorbed at the surface. Journal of Geophysical Research, 102, 29 717-29 727.

Li, Z., Nadon S., Cihlar J. (2000), Satellite detection of Canadian boreal forest fires: Development and application of an algorithm, International Journal of Remote Sensing, 21, 3057-3069.

Liou, K. N., 1992: Radiation and cloud processes in the atmosphere: Theory, observation, and modeling. Oxford University Press, New York.

Masonis, S. J., and Warren, S. G. (2001), Gain of the AVHRR visible channel as tracked using bidirectional reflectance of Antarctic and Greenland snow. International Journal of Remote Sensing, 22, 1495-1520

Nakajima, T., Higurashi, A., Kawamoto, K., Penner, J. E. (2001), A possible correlation between satellite-derived cloud and aerosol microphysical parameters. Geophyisical Research Letters, 28, 1171-1174.

Qin, Z., and Karnieli, A. (1999), Progress in the remote sensing of land surface temperature and ground emissivity using NOAA-AVHRR data. International Journal of Remote Sensing, 20, 2367-2393. 
Rao, C. R. N., and Chen, J. (1999), Revised post-launch calibration of the visible and nearinfrared channels of the Advanced Very High Resolution Radiometer (AVHRR) on the NOAA-14 spacecraft. International Journal of Remote Sensing, 20, 3485-3491.

Reynolds, R.W., Smith, T.M. (1993), Improved global sea surface temperature analysis using optimum interpolation. Journal of Climate, 7, 929-948.

Rossow, W.B. (1989), Measuring cloud properties from space: A review. Journal of Climate, 2, 201-213.

Rossow, W. B., and Schiffer, R. A. (1999), Advances in understanding clouds from ISCCP. Bulletin of the American Meteorological Society, 80, 2261-2287.

Rutan, D., and Charlock, T. P. (1997), Spectral reflectance, directional reflectance, and broad-band albedo of the earth's surface. Proc. $9^{\text {th }}$ Conference on Atmospheric Radiation, Long Beach, California, pp. 466-470.

Secker, J., Staenz, K., Budkewitsch, P., Neville, R. A. (1999), A vicarious calibration of the Probe-1 hyperspectral sensor; $4^{\text {th }}$ International Airborne Remote Sensing Conference and Exhibition/21st Canadian Symposium on Remote Sensing, Ottawa, Ontario, Canada, 2124 June , 1999.

Stowe, L. L., Ignatov, A. M., and Singh, R. R. (1997), Development, validation, and potential enhancements to the second-generation operational aerosol product at the National Environment Satellite, Data, and Information Service of the National Oceanic and Atmospheric Administration. Journal of Geophysical Research, 102, 16,923-16,934.

Tahnk, W. R., and Coakley, J. A. (2001), Updated calibration coefficients for NOAA-14 AVHRR channels 1 and 2. International Journal of Remote Sensing, 22, 15, 3053-3057 
Teillet, P. M., Staenz, K., and Williams, D. J. (1997), Effects of spectral, spatial, and radiometric characteristics on remote sensing vegetation indices of forested regions. Remote Sensing of Environment, 61, 139-149.

Teillet, P. M., Barker J., Markham, B.L., Irish R. R., Fedosejevs G., Storey J. C. (2000), Radiometric cross-calibration of the Landsat-7 ETM+ and Landsat-5 TM sensors based on tandem data sets. Remote Sensing of. Environment, 78, 39-54.

Trishchenko, A. P., Li, Z., Park, W., Cihlar, J. (2001), Corrections for the BRDF and topographic effects in satellite retrieval of surface spectral reflectance in solar spectral region. In Proc. of the Int. Radiation Symp.IRS 2000. St.Petersburg, Russia, August 2000. W.L. Smith and Y. Tymofeyev (eds). A. Deepak, v. 147: Current Problems in Atmospheric Radiation. (in press).

Vermote, E., Tanré, D., Deuzé, J. L., Herman, M., and Morcette, J. J. (1997), Second simulation of the satellite signal in the solar spectrum: An overview. IEEE Transactions on Geoscience and Remote Sensing, 35, 675-686. 
Table 1. Surface types assumed in this study.

\begin{tabular}{|c|c|}
\hline Surface types & Surface types \\
\hline 1. Coniferous Forest ${ }^{(1,2)}$ & 7. Cropland \\
\hline 2. Deciduous Broadleaf Forest ${ }^{(1,2)}$ & 8. Crop/Natural Veg. Mosaic \\
\hline 3. Closed Shrubland & 9. Barren/Desert \\
\hline 4. Open Shrubland & 10. Water Bodies \\
\hline 5. Drygrass/savanna & 11. Fresh Snow \\
\hline 6. Grassland ${ }^{(1-4)}$ & 12. Coarse Granular Snow \\
\hline
\end{tabular}

Superscripts in Table 1 denote the number of spectral curves used in simulations (Figure 2). 


\section{Table 2.}

Parameters of quadratic best fit to absolute spectral correction $\Delta \rho=\rho-\rho_{\text {NOAA-9 }}$ and relative spectral correction $\Delta \rho=\frac{\rho-\rho_{\text {NOAA-9 }}}{\rho_{\text {NOAA-9 }}}(\%)$ for visible (red) channel. Surface level. X denotes NDVI for particular sensor computed at the surface level.

\begin{tabular}{|c|c|c|c|c|c|c|}
\hline Instrument & Absolute correction & $\mathrm{r}^{2}$ & $\sigma$ & Relative correction (\%) & $\mathrm{r}^{2}$ & $\sigma(\%)$ \\
\hline AVHRR/ N-6 & $0.00035-0.0189 \mathrm{X}+0.0141 \mathrm{X}^{2}$ & 0.80 & 0.0013 & $-0.160-0.445 \mathrm{X}-19.525 \mathrm{X}^{2}$ & 0.97 & 0.97 \\
\hline AVHRR/ N-7 & $0.00026-0.0153 \mathrm{X}+0.0127 \mathrm{X}^{2}$ & 0.77 & 0.0010 & $0.108-2.230 \mathrm{X}-11.050 \mathrm{X}^{2}$ & 0.96 & 0.76 \\
\hline AVHRR/ N-8 & $-0.00056+0.0014 \mathrm{X}+0.0033 \mathrm{X}^{2}$ & 0.77 & 0.0007 & $0.087-9.037 \mathrm{X}+21.721 \mathrm{X}^{2}$ & 0.81 & 1.37 \\
\hline AVHRR/ N-10 & $0.00037-0.0195 \mathrm{X}+0.0153 \mathrm{X}^{2}$ & 0.79 & 0.0014 & $-0.159-1.411 \mathrm{X}-16.949 \mathrm{X}^{2}$ & 0.96 & 1.00 \\
\hline AVHRR/ N-11 & $0.00001-0.0012 \mathrm{X}+0.0005 \mathrm{X}^{2}$ & 0.76 & 0.0002 & $0.006+0.0744 \mathrm{X}-2.335 \mathrm{X}^{2}$ & 0.96 & 0.12 \\
\hline AVHRR/ N-12 & $-0.00022+0.0027 \mathrm{X}-0.0035 \mathrm{X}^{2}$ & 0.21 & 0.0005 & $0.073+0.1604 \mathrm{X}-0.947 \mathrm{X}^{2}$ & 0.14 & 0.47 \\
\hline AVHRR/ N-14 & $-0.00046+0.0112 \mathrm{X}-0.0077 \mathrm{X}^{2}$ & 0.82 & 0.0008 & $0.116-2.951 \mathrm{X}+18.076 \mathrm{X}^{2}$ & 0.94 & 0.87 \\
\hline AVHRR/ N-15 & $0.00029-0.0222 \mathrm{X}+0.0117 \mathrm{X}^{2}$ & 0.81 & 0.0021 & $-0.105+3.115 \mathrm{X}-36.306 \mathrm{X}^{2}$ & 0.98 & 1.31 \\
\hline AVHRR/ N-16 & $0.00028-0.0217 \mathrm{X}+0.0123 \mathrm{X}^{2}$ & 0.80 & 0.0020 & $-0.096+2.044 \mathrm{X}-32.746 \mathrm{X}^{2}$ & 0.98 & 1.26 \\
\hline MLODIS & $-0.00037-0.0118 \mathrm{X}-0.0051 \mathrm{X}^{2}$ & 0.73 & 0.0035 & $0.046+12.136 \mathrm{X}-56.504 \mathrm{X}^{2}$ & 0.98 & 1.87 \\
\hline VGT/SPOT & $-0.00086+0.0305 \mathrm{X}-0.0404 \mathrm{X}^{2}$ & 0.42 & 0.0034 & $0.570+16.234 \mathrm{X}-27.183 \mathrm{X}^{2}$ & 0.58 & 2.29 \\
\hline
\end{tabular}


Table 3.

Similar to Table 2, but for NIR channel. Surface level.

\begin{tabular}{|c|c|c|c|c|c|c|}
\hline Instrument & Absolute correction & $\mathrm{r}^{2}$ & $\sigma$ & Relative correction (\%) & $\mathrm{r}^{2}$ & $\sigma(\%)$ \\
\hline AVHRR/ N-6 & $-0.00069+0.00443 \mathrm{X}-0.0021 \mathrm{X}^{2}$ & 0.79 & 0.0005 & $-0.0777+0.8707 \mathrm{X}-0.2788 \mathrm{X}^{2}$ & 0.95 & 0.05 \\
\hline AVHRR/ N-7 & $-0.00049+0.00142 \mathrm{X}-0.0045 \mathrm{X}^{2}$ & 0.77 & 0.0004 & $-0.0682-0.2536 \mathrm{X}-0.5465 \mathrm{X}^{2}$ & 0.90 & 0.08 \\
\hline AVHRR/ N-8 & $0.00005-0.00385 \mathrm{X}-0.002 \mathrm{X}^{2}$ & 0.88 & 0.0006 & $-0.0205-1.5927 \mathrm{X}+0.1487 \mathrm{X}^{2}$ & 0.96 & 0.10 \\
\hline AVHRR/ N-10 & $-0.00073+0.00745 \mathrm{X}+0.0031 \mathrm{X}^{2}$ & 0.89 & 0.0012 & $-0.0539+2.3498 \mathrm{X}+0.3563 \mathrm{X}^{2}$ & 0.98 & 0.13 \\
\hline AVHRR/ N-11 & $-0.00008-0.0001 \mathrm{X}+0.0002 \mathrm{X}^{2}$ & 0.11 & 0.0001 & $-0.0140-0.1087 \mathrm{X}+0.1505 \mathrm{X}^{2}$ & 0.19 & 0.02 \\
\hline AVHRR/ N-12 & $-0.00025+0.00264 \mathrm{X}+0.0014 \mathrm{X}^{2}$ & 0.90 & 0.0004 & $-0.0184+0.8199 \mathrm{X}+0.2289 \mathrm{X}^{2}$ & 0.98 & 0.04 \\
\hline AVHRR/ N-14 & $-0.00335+0.02615 \mathrm{X}-0.0168 \mathrm{X}^{2}$ & 0.74 & 0.0026 & $-0.3457+5.4112 \mathrm{X}-3.1057 \mathrm{X}^{2}$ & 0.89 & 0.33 \\
\hline AVHRR/ N-15 & $-0.00082+0.01153 \mathrm{X}+0.0051 \mathrm{X}^{2}$ & 0.88 & 0.0020 & $-0.03087+4.0655 \mathrm{X}+0.210 \mathrm{X}^{2}$ & 0.96 & 0.29 \\
\hline AVHRR/ N-16 & $-0.00164+0.01696 \mathrm{X}+0.0002 \mathrm{X}^{2}$ & 0.86 & 0.0023 & $-0.12313+5.0171 \mathrm{X}-0.669 \mathrm{X}^{2}$ & 0.96 & 0.32 \\
\hline MLI/ADEOS & $0.0056-0.021 \mathrm{X}+0.0605 \mathrm{X}^{2}$ & 0.73 & 0.0070 & $0.6173+2.1544 \mathrm{X}+8.6714 \mathrm{X}^{2}$ & 0.83 & 1.52 \\
\hline VGT/SPOT & $0.00349-0.00826 \mathrm{X}+0.0532 \mathrm{X}^{2}$ & 0.81 & 0.0059 & $0.4960+4.1976 \mathrm{X}+7.6205 \mathrm{X}^{2}$ & 0.88 & 1.25 \\
\hline
\end{tabular}




\section{Table 4}

Similar to Table 2-3, but for NDVI. Surface level.

\begin{tabular}{|c|c|c|c|c|c|c|}
\hline Instrument & Absolute correction & $\mathrm{r}^{2}$ & $\sigma$ & Relative correction (\%) & $\mathrm{r}^{2}$ & $\sigma(\%)$ \\
\hline AVHRR/ N-6 & $0.00005+0.052 \mathrm{X}-0.02278 \mathrm{X}^{2}$ & 0.97 & 0.0021 & $3.84+3.7437 \mathrm{X}-5.227 \mathrm{X}^{2}$ & 0.43 & 0.48 \\
\hline AVHRR/ N-7 & $0.00001+0.03632 \mathrm{X}-0.0196 \mathrm{X}^{2}$ & 0.93 & 0.0018 & $3.315-0.3486 \mathrm{X}-1.459 \mathrm{X}^{2}$ & 0.50 & 0.50 \\
\hline AVHRR/ N-8 & $0.00006-0.0018 \mathrm{X}-0.0205 \mathrm{X}^{2}$ & 0.88 & 0.0021 & $-3.0562+9.4069 \mathrm{X}-10.072 \mathrm{X}^{2}$ & 0.50 & 0.84 \\
\hline AVHRR/ N-10 & $0.0002+0.0648 \mathrm{X}-0.0372 \mathrm{X}^{2}$ & 0.97 & 0.0020 & $5.301+2.526 \mathrm{X}-5.774 \mathrm{X}^{2}$ & 0.77 & 0.45 \\
\hline AVHRR/ N-11 & $-0.0001+0.0031 \mathrm{X}+0.00072 \mathrm{X}^{2}$ & 0.87 & 0.0005 & $-0.1952+1.601 \mathrm{X}-1.137 \mathrm{X}^{2}$ & 0.47 & 0.25 \\
\hline AVHRR/ N-12 & $-0.00032+0.0031 \mathrm{X}+0.0007 \mathrm{X}^{2}$ & 0.39 & 0.0015 & $-1.316+5.1 \mathrm{X}-3.795 \mathrm{X}^{2}$ & 0.44 & 0.82 \\
\hline AVHRR/ N-14 & $-0.00201+0.0099 \mathrm{X}-0.0304 \mathrm{X}^{2}$ & 0.93 & 0.0013 & $6.986-34.3909 \mathrm{X}+30.8637 \mathrm{X}^{2}$ & 0.83 & 1.81 \\
\hline AVHRR/ N-15 & $-0.00026+0.0877 \mathrm{X}-0.0307 \mathrm{X}^{2}$ & 0.97 & 0.0038 & $5.4+10.478 \mathrm{X}-10.894 \mathrm{X}^{2}$ & 0.57 & 0.82 \\
\hline AVHRR/ N-16 & $-0.00061+0.091 \mathrm{X}-0.0391 \mathrm{X}^{2}$ & 0.97 & 0.0034 & $8.139-0.4926 \mathrm{X}-2.1904 \mathrm{X}^{2}$ & 0.29 & 1.25 \\
\hline MLI/ADEOS & $-0.00086+0.0295 \mathrm{X}+0.0667 \mathrm{X}^{2}$ & 0.81 & 0.0149 & $-20.982+97.84 \mathrm{X}-74.127 \mathrm{X}^{2}$ & 0.89 & 4.62 \\
\hline VGT/SPOT & $-0.0006-0.0153 \mathrm{X}+0.05836 \mathrm{X}^{2}$ & 0.54 & 0.0104 & $-15.758+61.013 \mathrm{X}-47.087 \mathrm{X}^{2}$ & 0.82 & 3.77 \\
\hline
\end{tabular}




\section{Table 5.}

Parameters of polynomial fit to the relative spectral correction $\Delta \rho=\frac{\rho-\rho_{\text {NOAA-9 }}}{\rho_{\text {NOAA-9 }}}(\%)$ for visible (red) channel. Top-of-the-atmosphere level. X denotes NDVI for particular sensor computed at the TOA level.

\begin{tabular}{|c|c|c|c|}
\hline Instrument & Relative correction (\%) & $\mathrm{r}^{2}$ & $\sigma(\%)$ \\
\hline AVHRR/ N-6 & $-0.01588-4.62556 \mathrm{X}-7.96852 \mathrm{X}^{2}$ & 0.93 & 0.77 \\
\hline AVHRR/ N-7 & $0.04201-4.12357 \mathrm{X}-3.67224 \mathrm{X}^{2}$ & 0.88 & 0.68 \\
\hline AVHRR/ N-8 & $-0.41911-2.87354 \mathrm{X}+8.89133 \mathrm{X}^{2}$ & 0.65 & 0.60 \\
\hline AVHRR/ N-10 & $0.01277-4.84277 \mathrm{X}-6.23082 \mathrm{X}^{2}$ & 0.90 & 0.84 \\
\hline AVHRR/ N-11 & $-0.01258-0.48667 \mathrm{X}-1.27999 \mathrm{X}^{2}$ & 0.94 & 0.09 \\
\hline AVHRR/ N-12 & $-0.09189-0.2534 \mathrm{X}-1.66716 \mathrm{X}^{2}$ & 0.53 & 0.35 \\
\hline AVHRR/ N-14 & $-0.15411+1.30802 \mathrm{X}+7.27126 \mathrm{X}^{2}$ & 0.92 & 0.47 \\
\hline AVHRR/ N-15 & $0.10932-6.31443 \mathrm{X}-18.25781 \mathrm{X}^{2}$ & 0.97 & 0.98 \\
\hline AVHRR/ N-16 & $0.04244-6.30091 \mathrm{X}-16.02976 \mathrm{X}^{2}$ & 0.96 & 0.96 \\
\hline MODIS & $1.37765+3.11606 \mathrm{X}-40.78357 \mathrm{X}^{2}$ & 0.96 & 1.71 \\
\hline VGT/SPOT & $1.01934+9.33224 \mathrm{X}-28.91508 \mathrm{X}^{2}$ & 0.70 & 2.38 \\
\hline GLI/ADEOS & $1.5794+6.87468 \mathrm{X}-49.41134 \mathrm{X}^{2}$ & 0.93 & 2.60 \\
\hline & & & \\
\hline & & & \\
\hline
\end{tabular}


Table 6.

Similar to Table 4, but for NIR channel reflectance at the TOA level.

\begin{tabular}{|c|c|c|c|}
\hline Instrument & Relative correction (\%) & $\mathrm{r}^{2}$ & $\sigma(\%)$ \\
\hline AVHRR/ N-6 & $1.13467+1.64781 \mathrm{X}-1.26708 \mathrm{X}^{2}$ & 0.72 & 0.17 \\
\hline AVHRR/ N-7 & $-0.05851-0.53685 \mathrm{X}-0.44445 \mathrm{X}^{2}$ & 0.89 & 0.08 \\
\hline AVHRR/ N-8 & $1.00893-1.28713 \mathrm{X}-0.49973 \mathrm{X}^{2}$ & 0.90 & 0.14 \\
\hline AVHRR/ N-10 & $0.67763+3.41197 \mathrm{X}-0.56705 \mathrm{X}^{2}$ & 0.95 & 0.21 \\
\hline AVHRR/ N-11 & $0.41666+0.15057 \mathrm{X}-0.16803 \mathrm{X}^{2}$ & 0.14 & 0.04 \\
\hline AVHRR/ N-12 & $0.65109+1.4419 \mathrm{X}-0.42437 \mathrm{X}^{2}$ & 0.92 & 0.10 \\
\hline AVHRR/ N-14 & $-0.06817+5.81785 \mathrm{X}-3.93554 \mathrm{X}^{2}$ & 0.89 & 0.36 \\
\hline AVHRR/ N-15 & $-0.07092+4.90503 \mathrm{X}-0.23422 \mathrm{X}^{2}$ & 0.96 & 0.30 \\
\hline AVHRR/ N-16 & $-0.62499+5.51994 \mathrm{X}-0.84905 \mathrm{X}^{2}$ & 0.96 & 0.30 \\
\hline MODIS & $16.69042+23.8168 \mathrm{X}-9.82829 \mathrm{X}^{2}$ & 0.72 & 3.10 \\
\hline VGT/SPOT & $10.65846+12.30469 \mathrm{X}+0.97572 \mathrm{X}^{2}$ & 0.80 & 1.88 \\
\hline GLI/ADEOS & $11.94219+9.91694 \mathrm{X}+2.78224 \mathrm{X}^{2}$ & 0.75 & 2.14 \\
\hline
\end{tabular}




\section{Table 7.}

Parameters of polynomial fit to the absolute spectral correction for NDVI. Top-of-theatmosphere level. X denotes NDVI for particular sensor computed at the TOA level.

\begin{tabular}{|c|c|c|c|}
\hline Instrument & Absolute correction (\%) & $\mathrm{r}^{2}$ & $\sigma(\%)$ \\
\hline AVHRR/ N-6 & $0.00659+0.0435 \mathrm{X}-0.02586 \mathrm{X}^{2}$ & 0.93 & 0.0023 \\
\hline AVHRR/ N-7 & $-0+0.02435 \mathrm{X}-0.0125 \mathrm{X}^{2}$ & 0.82 & 0.0023 \\
\hline AVHRR/ N-8 & $0.00668-0.00023 \mathrm{X}-0.02523 \mathrm{X}^{2}$ & 0.84 & 0.0019 \\
\hline AVHRR/ N-10 & $0.00431+0.05377 \mathrm{X}-0.03415 \mathrm{X}^{2}$ & 0.95 & 0.0022 \\
\hline AVHRR/ N-11 & $0.00224+0.00428 \mathrm{X}-0.00276 \mathrm{X}^{2}$ & 0.77 & 0.0004 \\
\hline AVHRR/ N-12 & $0.00383+0.00911 \mathrm{X}-0.00633 \mathrm{X}^{2}$ & 0.46 & 0.0017 \\
\hline AVHRR/ N-14 & $0.00003+0.01558 \mathrm{X}-0.03521 \mathrm{X}^{2}$ & 0.66 & 0.0018 \\
\hline AVHRR/ N-15 & $0.00112+0.08104 \mathrm{X}-0.02105 \mathrm{X}^{2}$ & 0.98 & 0.0032 \\
\hline AVHRR/ N-16 & $-0.00138+0.08156 \mathrm{X}-0.02569 \mathrm{X}^{2}$ & 0.98 & 0.0028 \\
\hline MODIS & $0.06948+0.16993 \mathrm{X}-0.13581 \mathrm{X}^{2}$ & 0.82 & 0.0105 \\
\hline VGT/SPOT & $0.04608+0.04565 \mathrm{X}-0.01774 \mathrm{X}^{2}$ & 0.35 & 0.0134 \\
\hline GLI/ADEOS & $0.04879+0.08439 \mathrm{X}-0.0035 \mathrm{X}^{2}$ & 0.71 & 0.0160 \\
\hline
\end{tabular}




\section{Figure captions.}

Figure 1. Spectral response functions of visible (red) and NIR channels for AVHRR NOAA-6 to NOAA-16 and MODIS, VGT and GLI. a) AVHRR/1. Morning satellites NOAA-6,-8,-10. b) AVHRR/2. Afternoon satellites NOAA-7, -9, -11, 14 and morning satellite NOAA-12. c) AVHRR/3 (NOAA-15, -16), VGT/SPOT and GLI/ADEOS-II. Typical spectral reflectance curve for green vegetation is shown on each panel.

Figure 2. Spectra of the surface targets used in simulations. They were normalized according to Eq. (1). Top panel (a) shows spectra of non-vegetated surfaces, bottom panel (b) shows spectra for vegetated surfaces.

Figure 3. Surface and TOA reflectances and NDVI for the AVHRR/NOAA-9 for selected spectra. AVHRR radiometer onboard NOAA-9 satellite is considered as the reference sensor following to ISCCP approach (Rossow and Schiffer, 1999). Visible (red), Near-IR reflectances and NDVI are presented in sequence top to bottom. Note the break in vertical scale between 0.4 and 0.8 for the visible channel.

Figure 4. Absolute (solid triangles) and relative (open circles) differences in visible (red) channel reflectances at the surface level with respect to AVHRR/NOAA-9. The left scale is for relative difference and the right scale is for absolute difference. All data points are plotted versus NDVI of particular sensor. Quadratic best fits for absolute (solid) and relative (dashed) differences are also shown. Parameters of fitting curves are given in Table 2.

Figure 5. Similar to Figure 4, but for the NIR channel at the surface level. Parameters of fitting curves are given in Table 3.

Figure 6. Similar to Figure 4 and 5, but for NDVI at the surface level. Parameters of fitting curves are given in Table 4. 
Figure 7. Absolute (solid triangles) and relative (open circles) differences in visible (red) channel reflectances at the TOA levels with respect to AVHRR/NOAA-9. Solid lines correspond to quadratic fit to relative difference. Parameters of fitting curves are given in Table 5.

Figure 8. Similar to Figure 7, but for NIR channel reflectances at the TOA level. Parameters of fitting curves are given in Table 6.

Figure 9. Similar to Figure 7 and 8, but for NDVI at the TOA level. Quadratic fit is plotted for absolute difference in NDVI due to spectral response function effect. Parameters of fitting curves are given in Table 7.

Figure 10. Sensitivity of spectral response function effect to aerosol optical depth $\tau$. Visible (red) channel reflectance at the TOA level. Aerosol optical depth for the basic atmospheric state is 0.06 , total water vapor is $1.5 \mathrm{~cm}$, ozone content is $350 \mathrm{DU}$. Computations were done for no aerosol $(\tau=0)$ and hazy ( $\tau=0.6)$ cases.

Figure 11. Top panel shows comparison of NDVI computed at the TOA level from AVHRR/NOAA-14 and AVHRR/NOAA-15. Small dots denote satellite observations. Open circles denote model simulations. Bottom panel shows distribution of difference in $\Delta$ NVI.

Figure 12. Similar to Figure 11, but for comparison of NDVI at the TOA level derived from MODIS and AVHRR/NOAA-14. Like in previous comparison, good agreement is found on average between modeling and observations both in the sign and magnitude of spectral response function effect. The scattering of observed points is due to possible residual cloud contamination and resampling of MODIS image from Integerized Sinusoidal Projection (ISP) to Lambert conformal conic projection, which alters the image resolution. 
Figure 1.

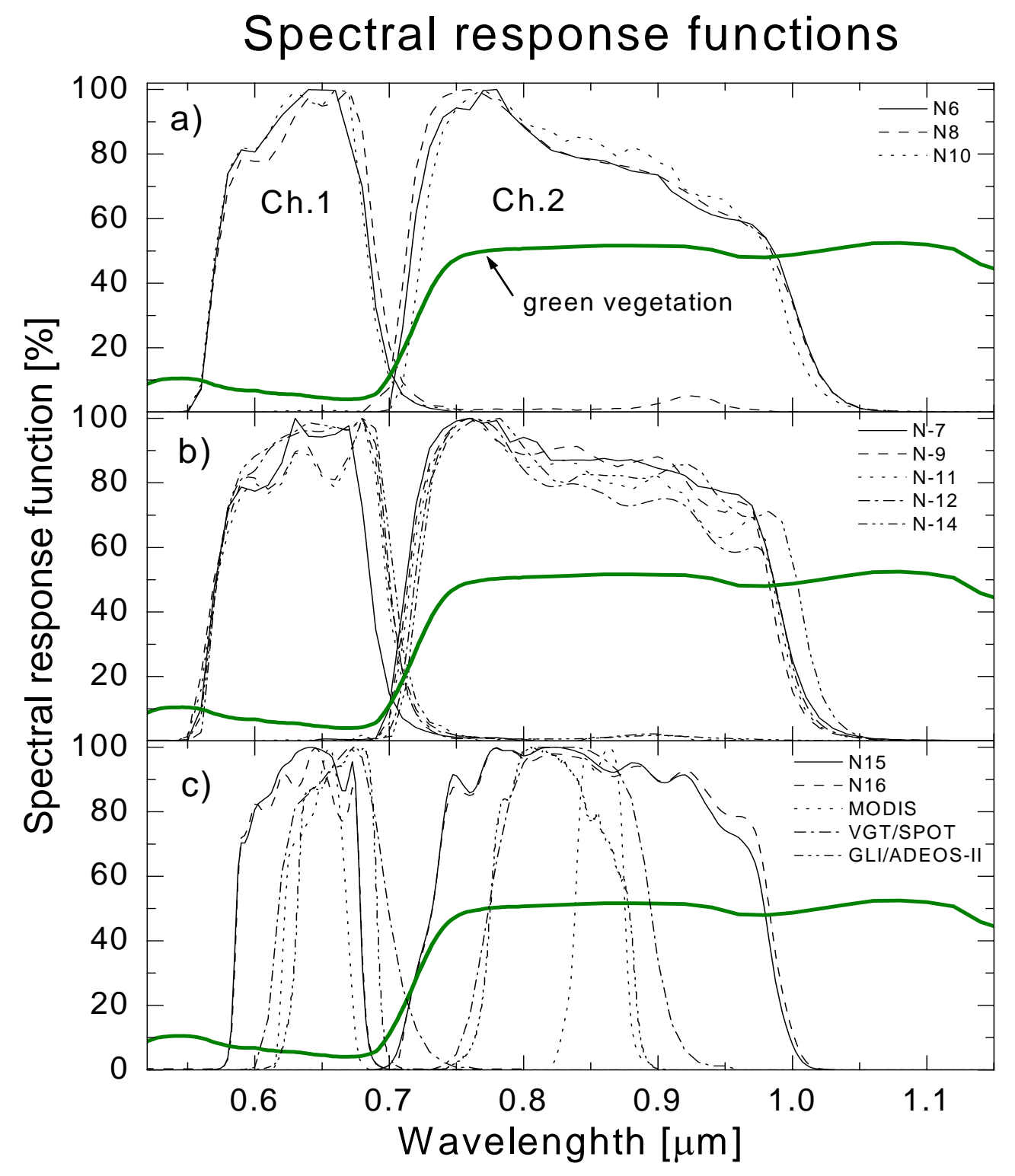

Figure 1 


\section{Figure 2.}

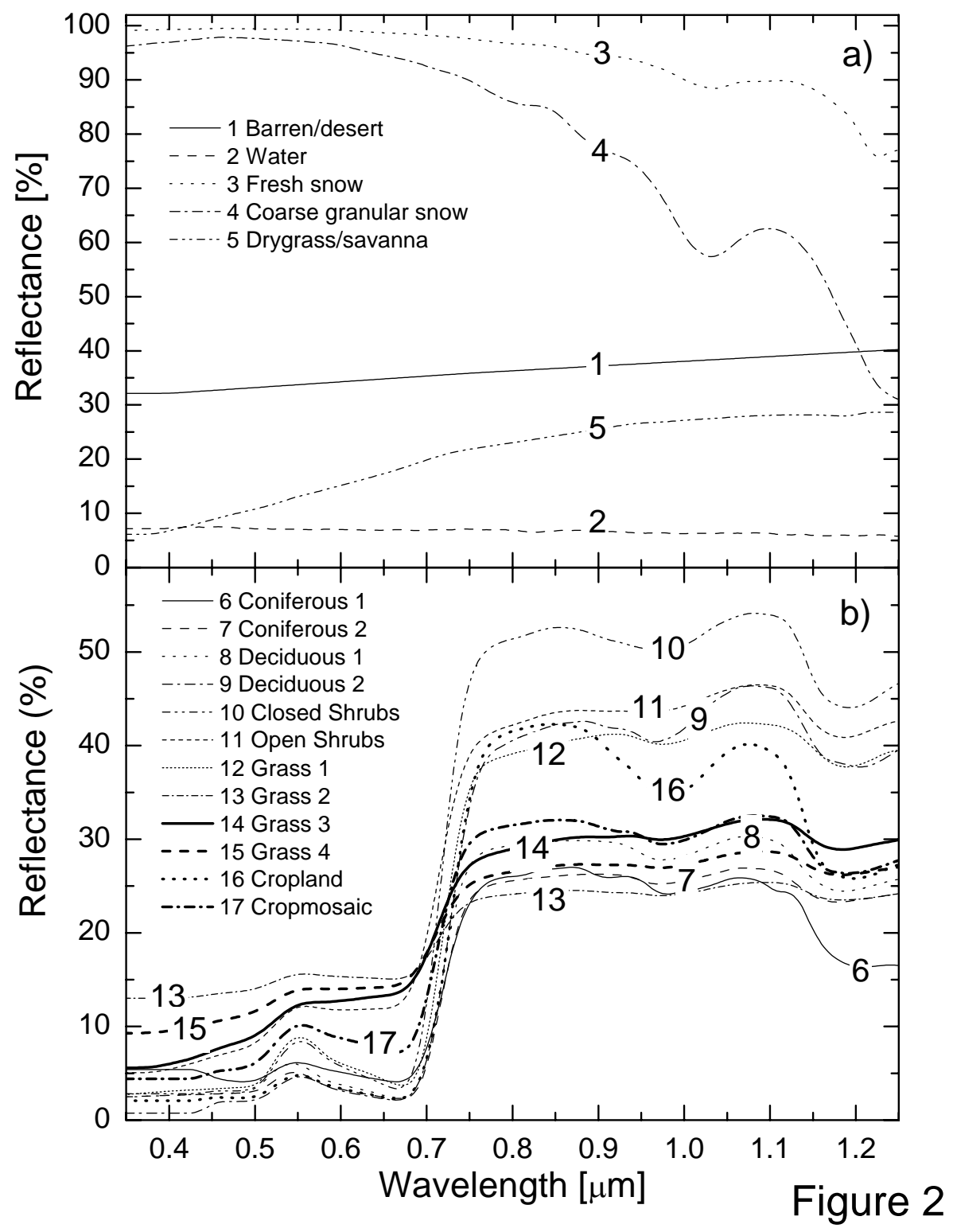


Figure 3.

Surface and TOA reflectances and NDVI for AVHRR/NOAA-9

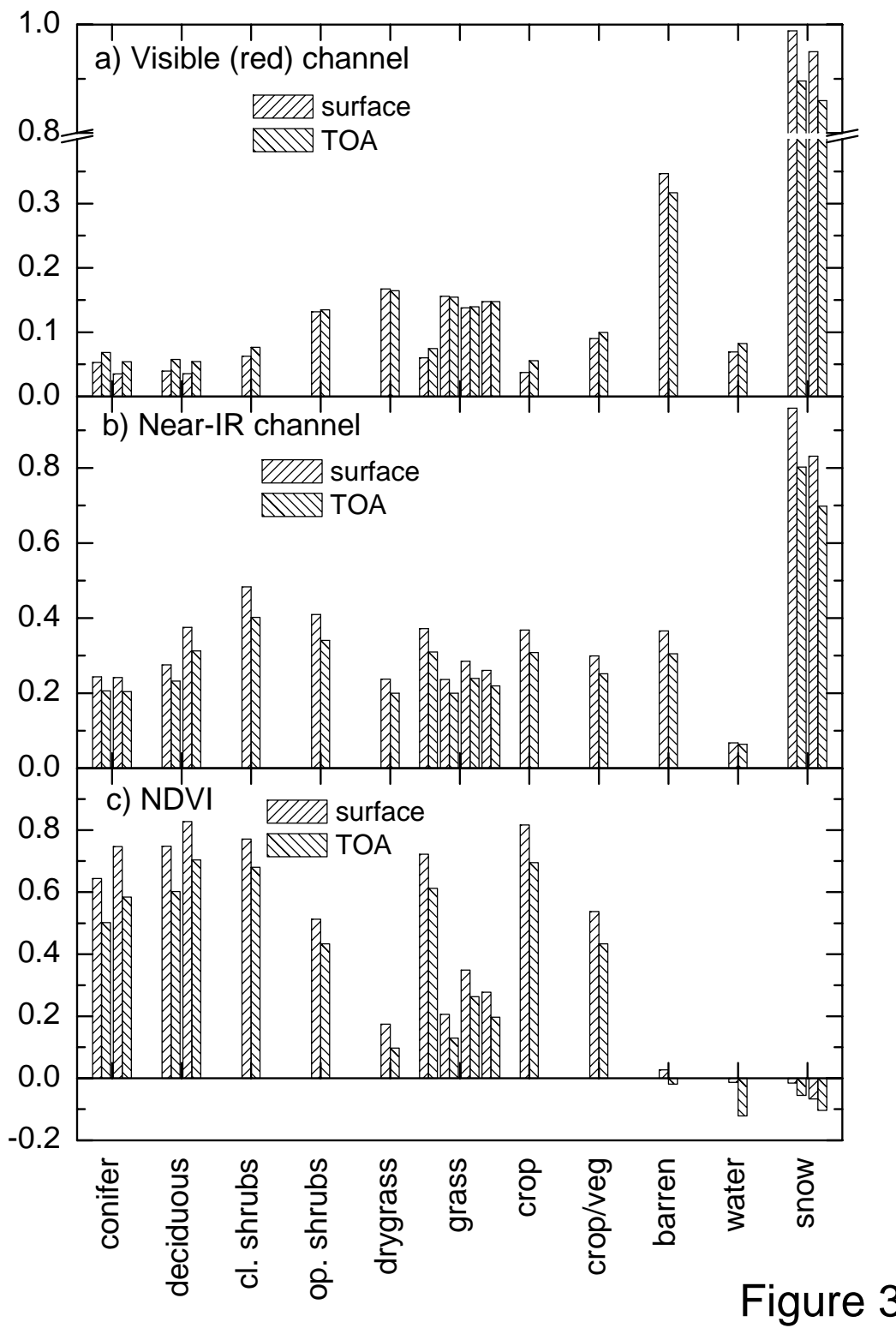


Figure 4.

Difference in surface reflectance for visible (red) channel relative to AVHRR/NOAA-9

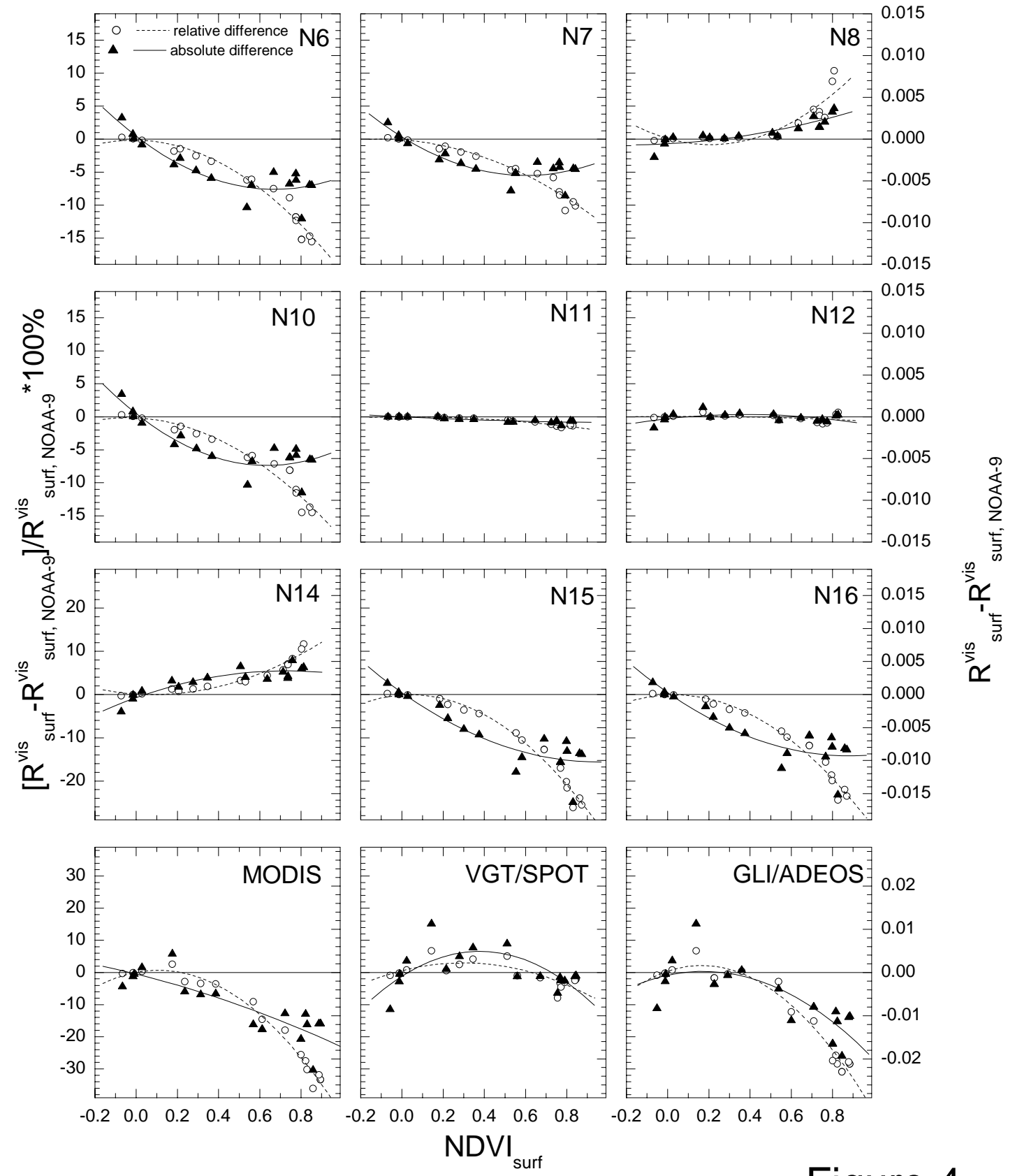

Figure 4 
Figure 5.

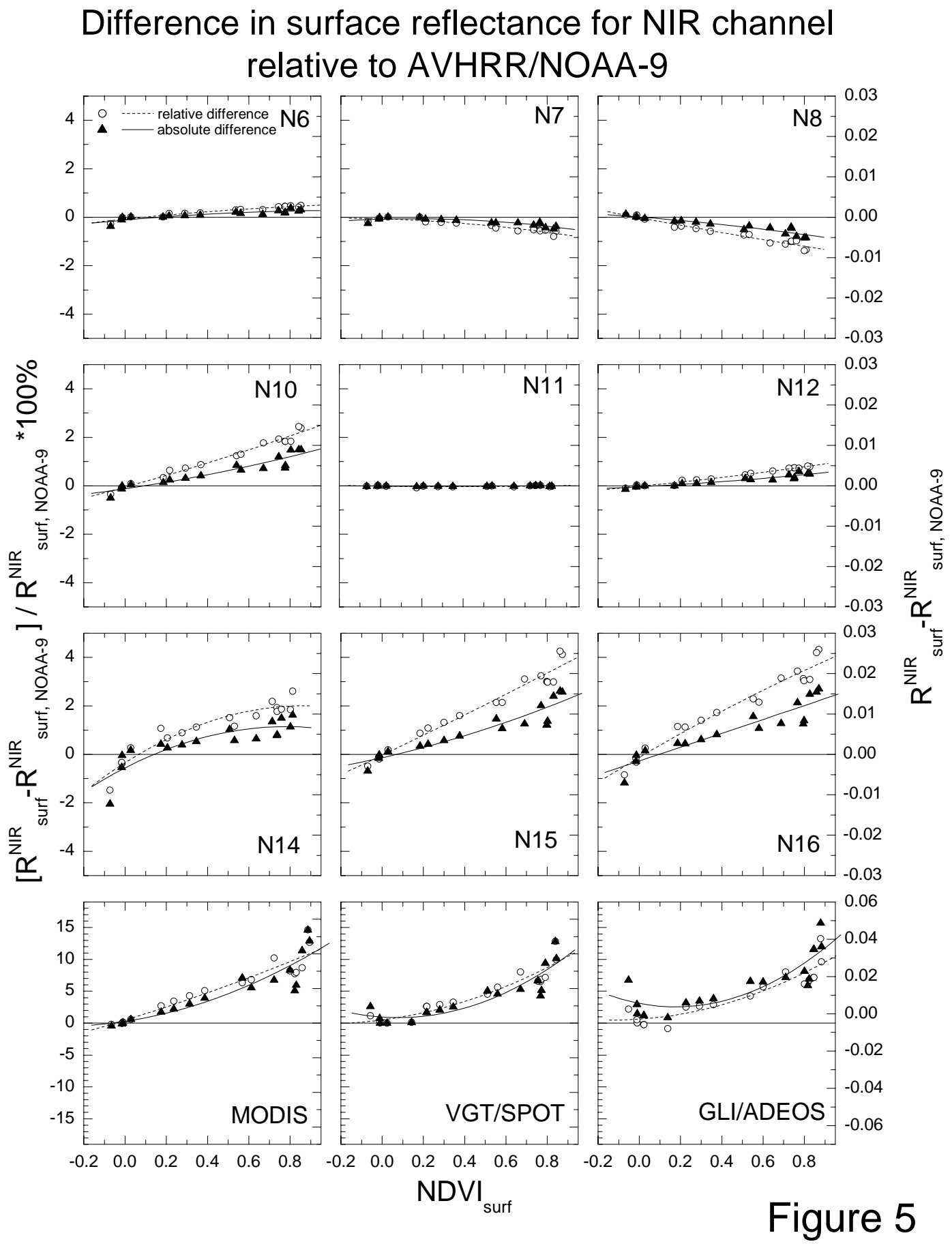


Figure 6.

Difference in NDVI computed at the surface level relative to AVHRR/NOAA-9

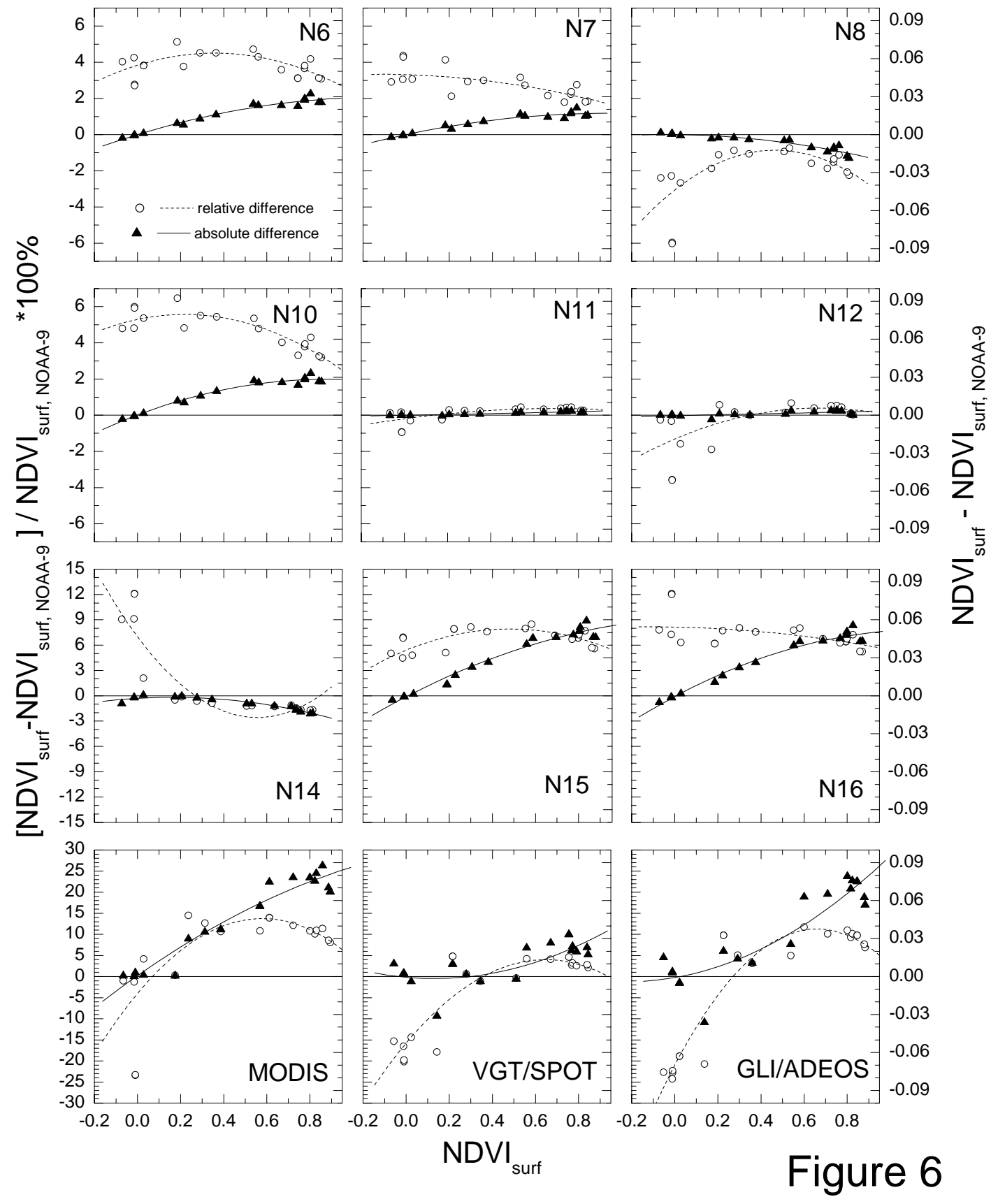


Figure 7.

\section{Difference in visible (red) channel TOA reflectance relative to AVHRR/NOAA-9}
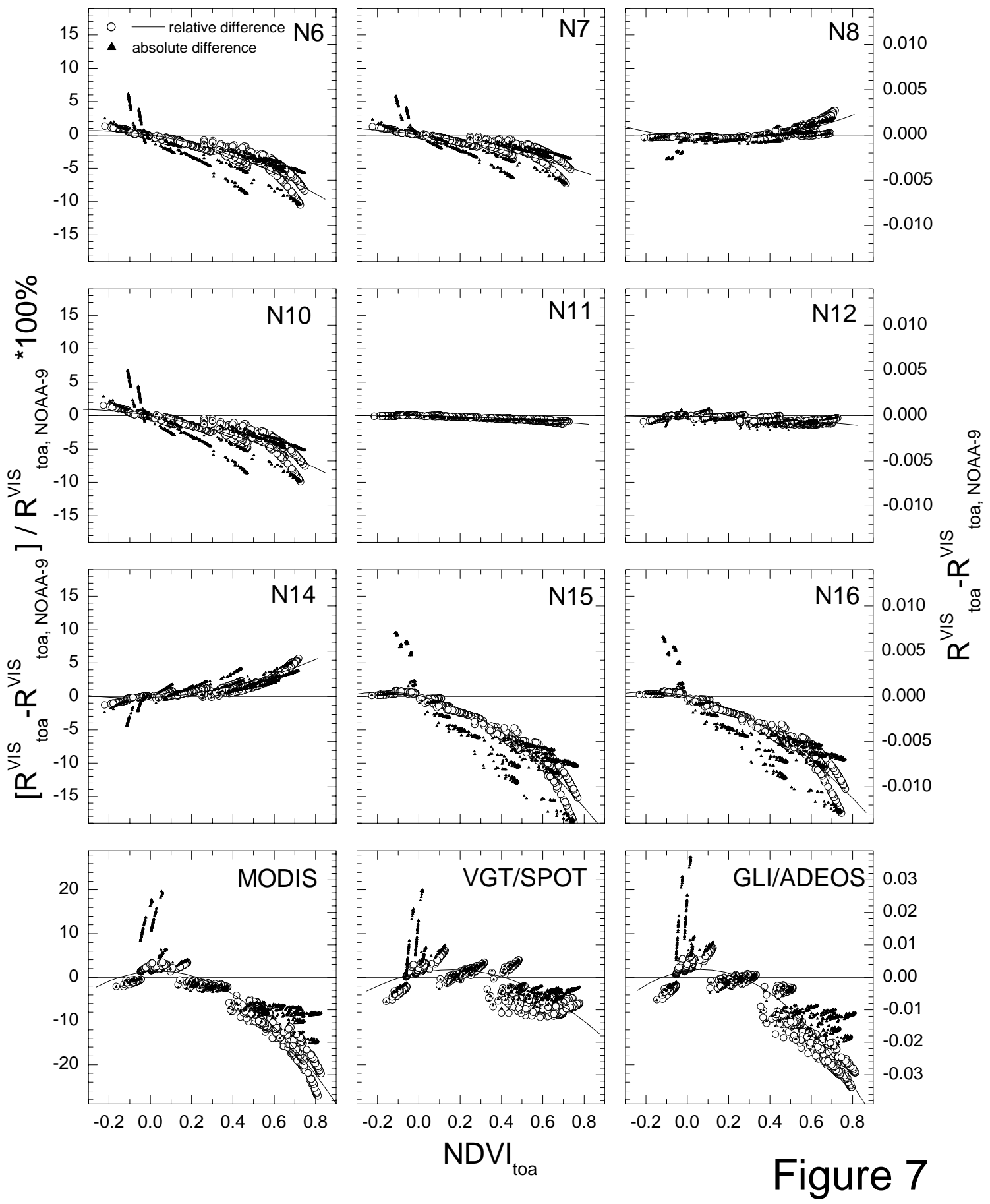
Figure 8.

\section{Difference in NIR channel TOA reflectance relative to AVHRR/NOAA-9}
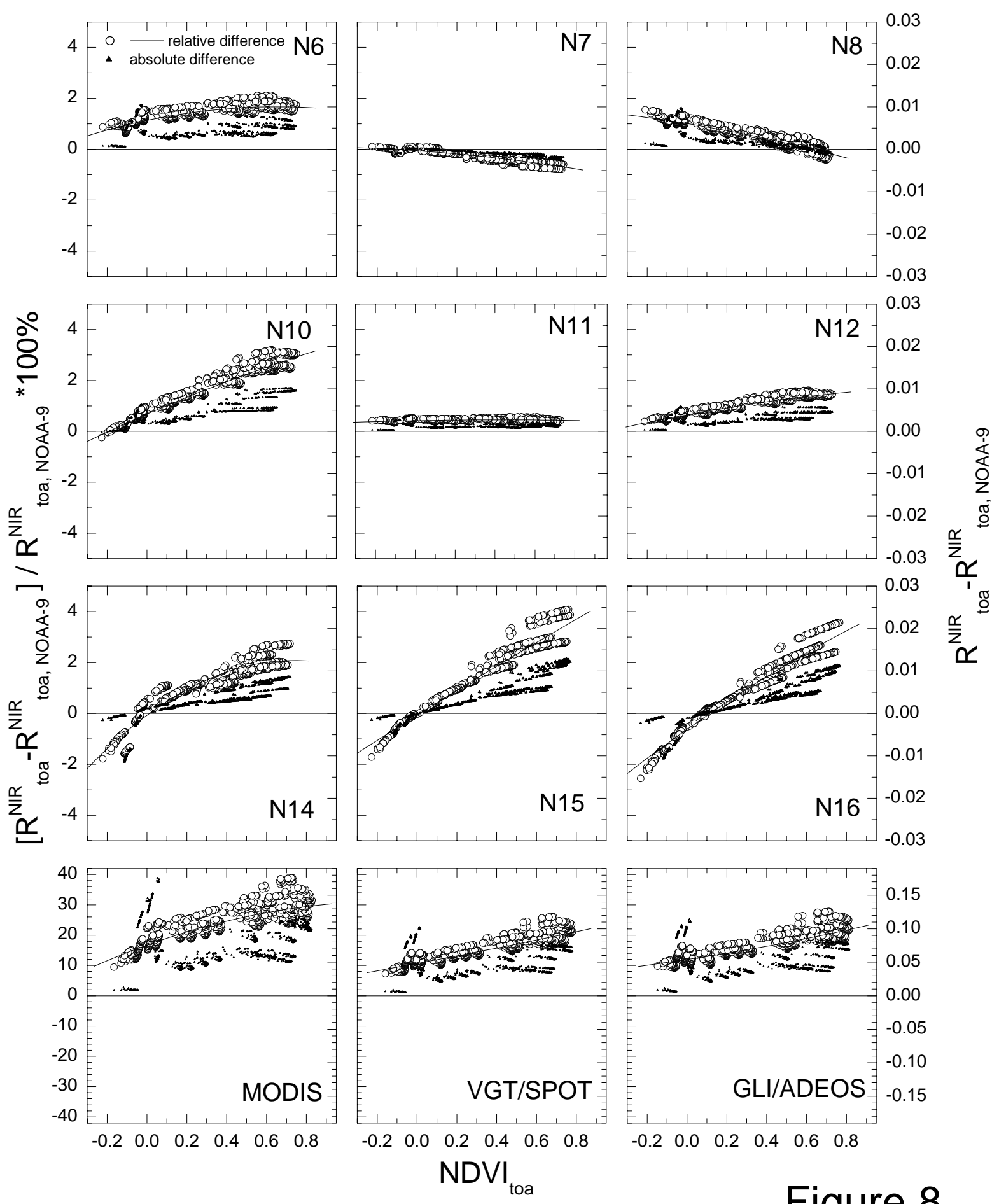

Figure 8 
Figure 9.

\section{Difference in NDVI computed at the TOA level relative to AVHRR/NOAA-9}
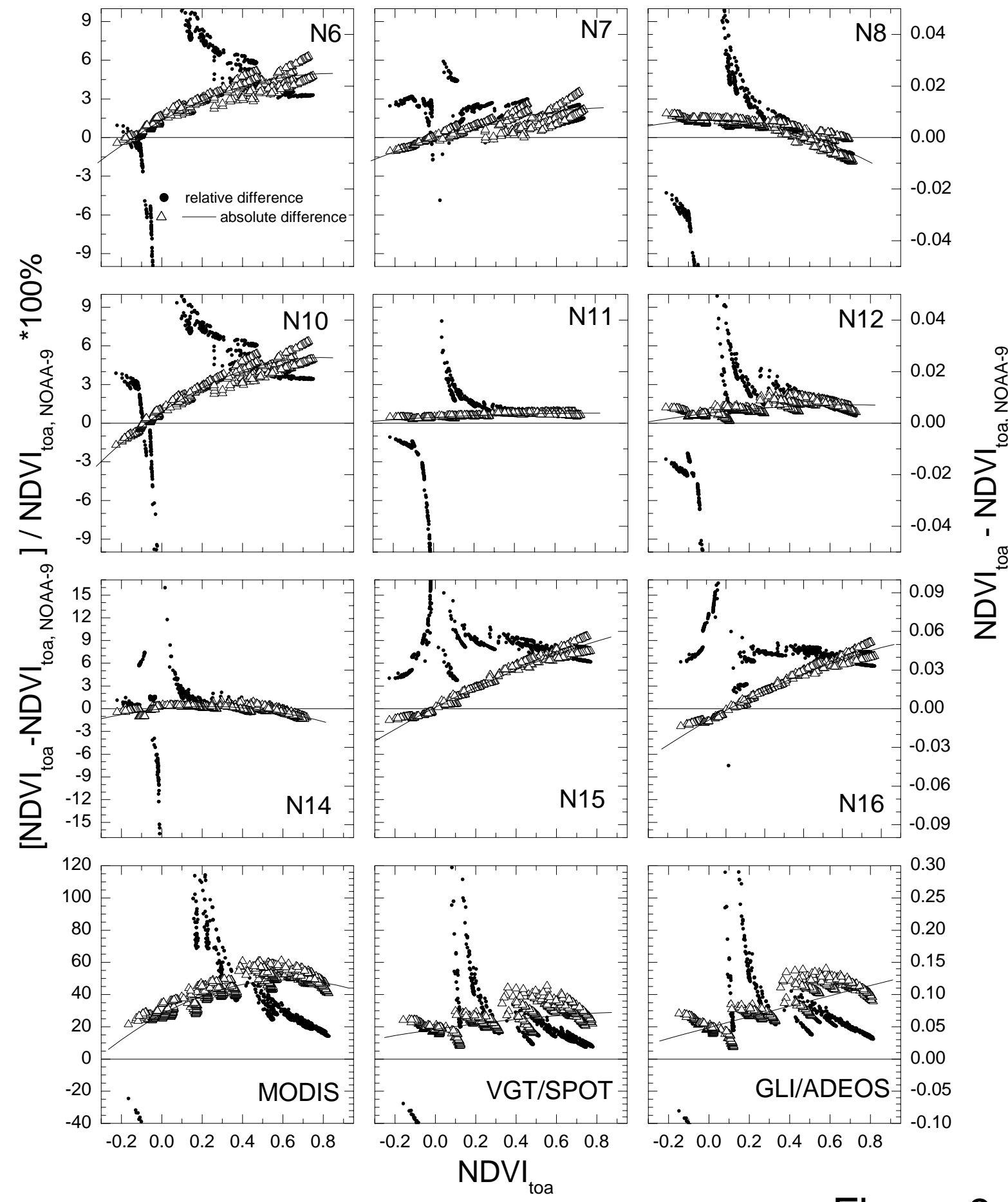

Figure 9 
Figure 10.

Sensitivity of spectral correction for visible (red) channel TOA reflectance to aerosol optical depth $\tau$. Basic state $\tau=0.06$

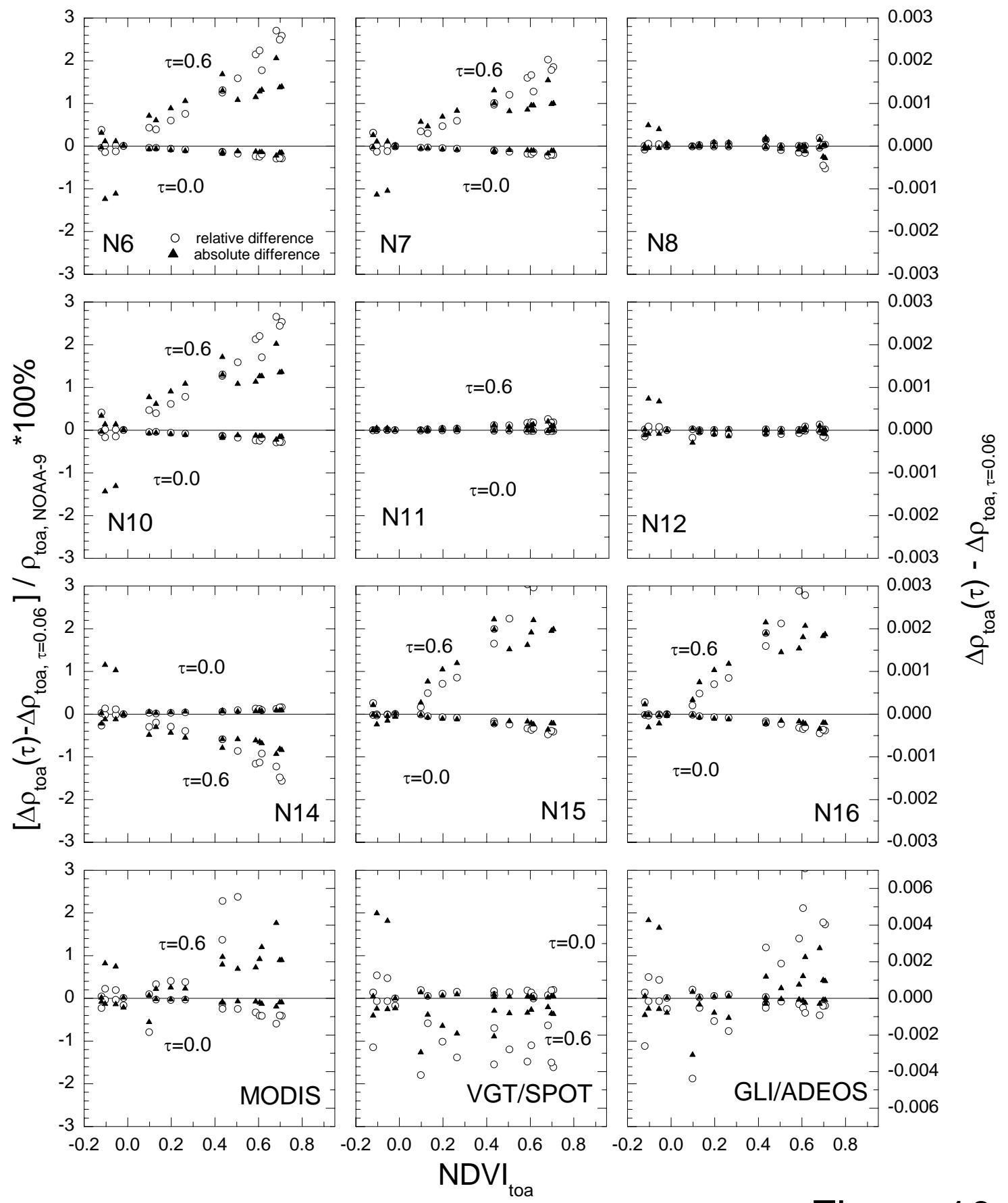

Figure 10 
Figure 11.
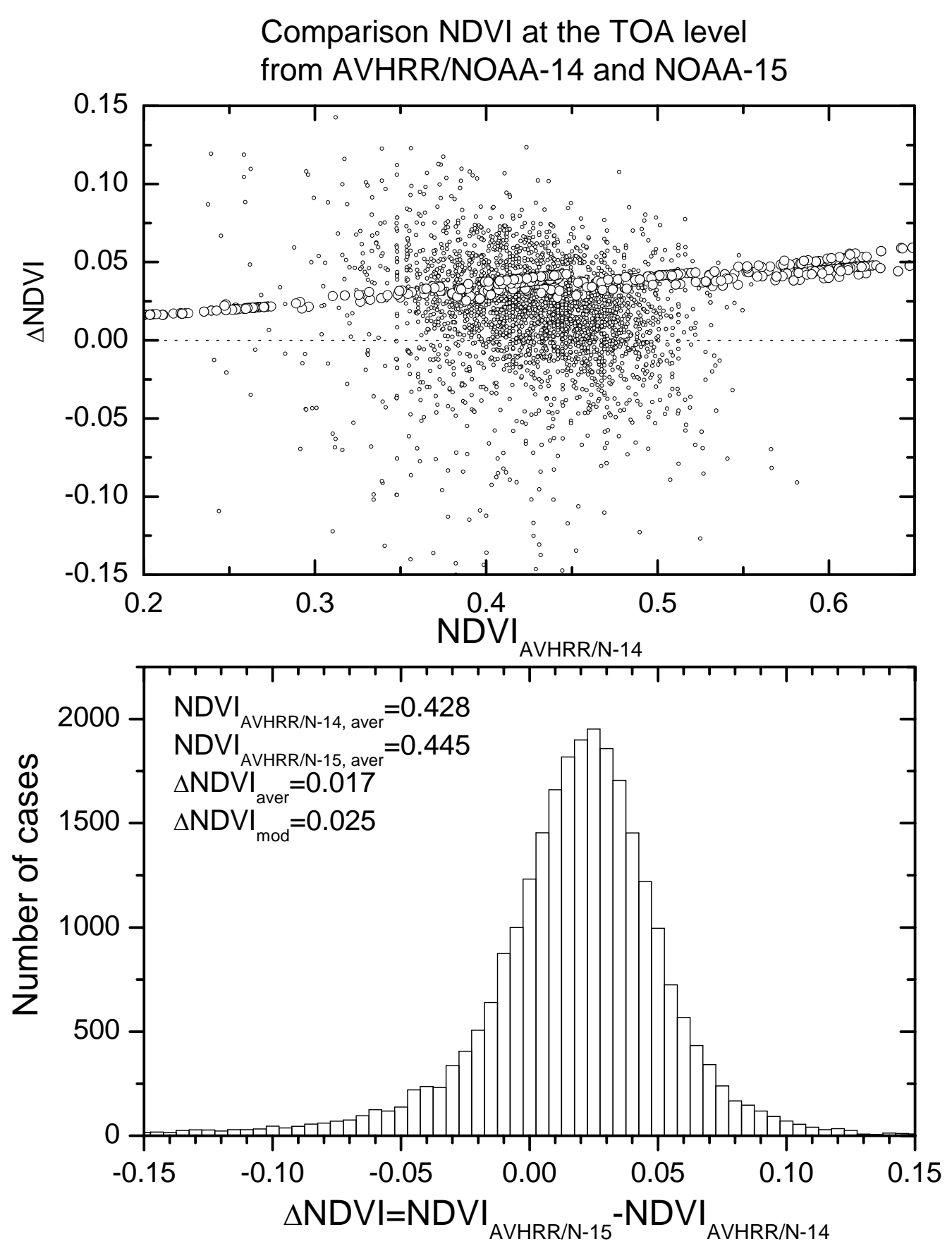

Figure 11 
Figure 12.
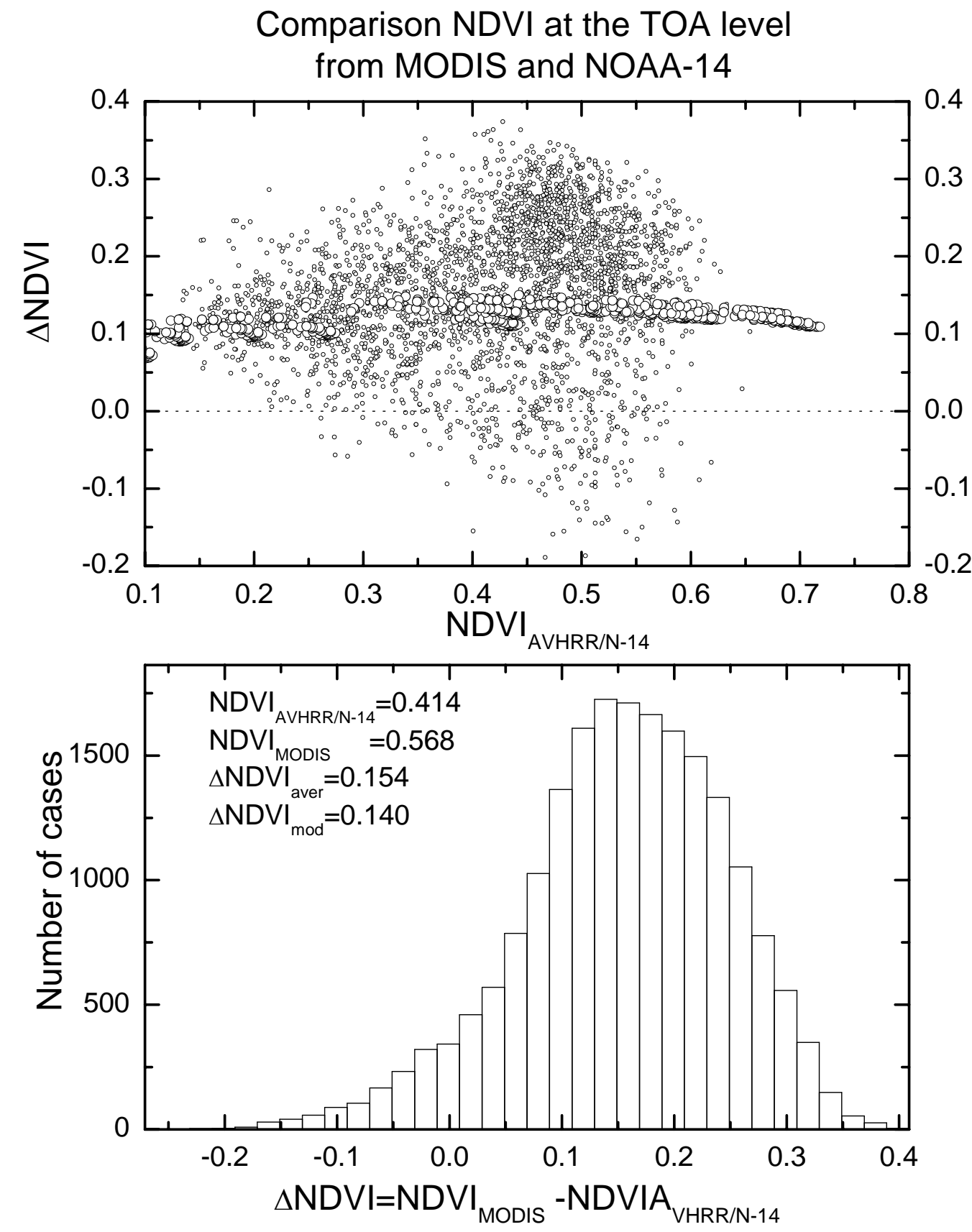

Figure 12 


\title{
Analyses of Sampling Errors in Solar Radiation Measurements in the Context of Model Validation over the Southern Great Plains Region
}

\author{
Zhanqing $\mathrm{Li}^{*}$, Maureen Cribb, Fu-Lung Chang \\ Department of Meteorology and Earth System Science Interdisciplinary Center \\ University of Maryland, College Park, MD 20742 \\ *Also affiliated with the Institute of Atmospheric Physics, Chinese Academy of Sciences \\ P.R. China \\ Alexander Trishchenko and Yi Luo \\ Applications Division, Canada Centre for Remote Sensing \\ Ottawa, Canada, K1A 0Y7
}

\author{
Submitted to the Special Issue on ARMCPM \\ Revised September 19, 2004 \\ Journal of Geophysical Research - Atmosphere
}




\section{Abstract}

For the general purpose of evaluating a model's performance, results from a hierarchy of models have been compared against radiation measurements made at the Atmospheric Radiation Measurement Southern Great Plains region. Important questions were raised as to 1) how well the measurements represent model grid mean values, 2) on what scale does modeling need to occur in order to capture a physical process, and 3) how much of model-observation differences are attributed to inherent sampling errors. We attempt to address some of these questions using surface solar radiation data obtained from ground and satellite. Using Geostationary Operational Environmental Satellite data, we mimic ground measurements at varying spatial density and temporal frequency. Observation uncertainties caused by cloud variability at different scales in different seasons were characterized, which can guide model-observation comparisons. In comparing single-point measurements against area mean satellite retrievals, much of the differences are caused by ground sampling errors. This implies that the inherent sampling errors must be taken into consideration when comparing model-simulated grid mean fluxes against ground-based point-specific observations. The sampling errors in different months, model domain sizes and integration intervals are quantified. For example, for March 2000, one may not expect model simulated instantaneous fluxes and the CF measurements to agree within $16 \mathrm{Wm}^{-2}$ for a model grid of $10 \times 10 \mathrm{~km}^{2}$; the best accuracy increases to $46 \mathrm{~W} \mathrm{~m}^{-2}$ and $64 \mathrm{~W} \mathrm{~m}^{-2}$ if the model grid increases to $200 \times 200 \mathrm{~km}^{2}$ and $400 \times 400 \mathrm{~km}^{2}$, respectively. The sampling uncertainties decrease rapidly as the averaging interval increases. For daily mean fluxes, for example, the sampling error is about $16 \mathrm{Wm}^{-2}$ in September 2000 over a typical general circulation model grid of $200 \times 200 \mathrm{~km}^{2}$. In general, for averaging intervals less than a day, the sampling error decreases sharply. Beyond one day, the sampling error levels off to a relatively 
small and stable value, implying that most clouds vary on the time scale of a day or a little longer. Of course, this time scale is dictated by the cloud system. The sampling error decreases as the number of ground stations increases within a domain, but the most substantial reduction occurs as the number of ground sites increases from 1 to 2 or 3 for a domain of $200 \times 200 \mathrm{~km}^{2}$. The shorter the averaging period, the more stations are needed to reduce the sampling error. Averaging over periods longer than 5 days reduces the error to a magnitude of less than $15 \mathrm{Wm}^{-2}$ over all domain sizes. While the statistics presented here vary with time and region, the method proposed should be valid in general. 


\section{Introduction}

Radiation measurements have been widely employed for evaluating cloud parameterization schemes and model simulation results [Morcrette, 2002]. Solar radiation is affected by numerous atmospheric (e.g. cloud) and surface (albedo) quantities that vary on a range of scales from thousands of kilometers to hundreds of meters [Rossow et al., 2002]. General circulation models (GCMs) typically have fixed grid cell sizes that range from tens to hundreds of kilometers, posing serious challenges in comparing observations to models [Randall et al., 2003]. For computational efficiency and accuracy, important subgrid details in cloud and radiation processes must be parameterized. Improving cloud and radiation parameterizations in GCMs is of paramount importance and has been the central goal of the Atmospheric Radiation Measurement (ARM) program of the United States' Department of Energy [Ackerman and Stokes, 2003; Stokes and Schwartz, 1994]. At present, the most critical requirements to advance the parameterization of radiative processes for use in GCMs are (1) quantitative description of the radiative energy under a wide range of meteorological conditions; (2) identification and investigation of the processes controlling the radiative balance, with a special emphasis on clouds; and (3) intensive measurements of cloud and radiation at a variety of temporal and physical scales.

To meet these needs, ARM has spearheaded major efforts to make observations on the scale of GCM gridboxes, so as to define the physics underlying some of the important parameterizations in GCMs. In the ARM Vision 2000 report, the modeling working group (MWG) recommended that more cloud and radiation data sets, incorporating more detailed information on their spatial and temporal distributions, be produced for direct use by the modeling community [Randall et al., 2000]. The inability of the MWG to test parameterizations 
of subgrid microphysical processes was attributed to insufficient subgrid measurements of cloud, surface and radiative quantities. This view was also echoed by the ARM Instantaneous Radiative Flux working group, which called for more emphasis on resolving spatial variability rather than focusing on point-specific measurements [Ellingson, 2000]. Given the long history of groundbased measurements in the ARM program and in other field programs, a major task is how to connect these local point-specific data with global models under a wide range of large-scale conditions [Randall et al., 2000].

Single-column modeling (SCM) has been the primary tool connecting GCMs and data collected in the field [Randall et al., 2003]. A SCM includes the bulk of the physics of a GCM and can be run in isolation from the rest of the GCM. In a SCM, a grid column of a climate model is isolated and supplemented with more detailed models of cloud and radiation processes. Data collected in the field can then be used to evaluate the performance of the improved parameterizations. However, the SCM suffers from the same coarse-resolution problem as a GCM when it comes to resolving cloud variability. A cloud system resolving model (CSRM) has high spatial and temporal resolutions and is useful for testing some radiative parameterizations over a wide range of space and time domains [Browning et al., 1993]. For example, several CSRM and SCMs are compared for multi-day simulations of convection during the TOGA-COARE experiment [Krueger and Lazarus, 2000]. Some CSRMs are now running at very high resolutions (down to a kilometer or less). As illustrated by Randall et al. [2003], field data can be used to combine CSRMs and SCMs in order to develop improved GCM parameterizations. In another study, Krueger and Burks [1998] developed and used an empirical relation between TOA pixel and surface pixel solar fluxes using satellite and point surface measurements to estimate 3-hourly area-averaged surface fluxes. 
The ARM program provides testbeds for different climate regimes. Of interest here is the Southern Great Plains (SGP) Cloud and Radiation Testbed site spanning from central Oklahoma northward into southern Kansas (Figure 1). This domain includes the Central Facility (CF) where the bulk of the instrumentation is located and twenty extended facilities spread non-uniformly throughout the SGP region which contain basic instrumentation such as meteorological profilers and radiometers. Despite this seemingly dense observation network, several outstanding questions still confront us. To name a few, 1) can cloud behavior be understood from the deployed instruments; 2) what type of measurements, and at what density are required to specify the boundary conditions for SCMs; 3) what, if any guideline, should we follow in adding observation stations and their locations for both permanent and mobile sites; and 4) at what scale does modeling need to occur in order to capture the physical properties that drive the system. To answer these questions, we need to understand system-scaling behavior due to inhomogeneous cloud and surface cover.

Solar radiation data from the Mesoscale Network of Oklahoma were analyzed for three months in 1994 to examine spatial representation of the radiation measurements at the CF [Barnett et al., 1996, 1998]. The sparsely distributed observation networks may resolve largescale variability, but are insufficient to gain statistics for comparison with high-resolution models such as the CSRM. Satellite data can be used to study the spacing of ground-based observation stations [Perez et al., 1997]. In this study, we take advantage of the high spatial and temporal resolution of the Geostationary Operational Environmental Satellites (GOES) dataset of cloud properties to mimic ground-based measurements of solar surface net radiation (SSNR) of varying density and temporal frequency and characterize their observation uncertainties caused by cloud variability at different scales in different seasons. Such scale-dependent statistics of observation 
uncertainties provide constraints on model-observation comparisons, and are thus valuable for improving and validating cloud parameterization schemes.

The following section introduces the retrieval models and input data used to obtain SSNR on different spatial and temporal scales. Section 3 presents the results concerning the observation uncertainties and their ramifications on determining the scales upon which useful validations can be performed for testing cloud parameterization schemes and model simulation results. Conclusions are contained in the final section.

\section{Models and input data}

Satellite inversion model

The SSNR was calculated using the models of Li et al. [1993] and Masuda et al. [1995]. The model of Li et al. [1993] essentially connects the net fluxes at the top of the atmosphere (TOA) and at the surface through a linear relationship that is a function of the TOA albedo, solar zenith angle and precipitable water amount. This relationship is independent of cloud optical thickness and surface albedo so that it can be used under both clear and cloudy sky conditions. The Li et al. model has been used to generate a global climatology of SSNR [Li and Leighton, 1993] with an accuracy of $5 \mathrm{Wm}^{-2}$ for monthly mean fluxes [Li et al., 1995]; good accuracies were also reported in other studies [Conant et al., 1997; Ramanathan et al., 1995; Waliser et al., 1996, 1999; Jing and Cess, 1998]. The model is currently used as one of the Cloud and the Earth’s Radiant Energy System algorithms generating the global SSNR [Wielicki et al., 1996].

For instantaneous and daily mean SSNR, larger errors are expected to occur due to a lack of the explicit inclusion of influential atmospheric factors such as cloud-top altitude and heavy aerosol loading [Li, 1998; Feng and Leighton, 2003]. Masuda et al. [1995] introduced 
corrections to the $\mathrm{Li}$ et al. [1993] algorithm to explicitly account for these two particular influences. Clouds at different altitudes affect atmospheric absorption, hence the SSNR, in different ways [Li and Moreau, 1996]. For low-altitude clouds, atmospheric solar absorption is enhanced compared to absorption under clear skies especially at large solar zenith angles. The reflection of incoming photons from clouds positioned higher in the atmosphere reduces the chances of absorption by water vapor and aerosol below the cloud, leading to a decrease in overall atmospheric solar absorption; this effect is strongest at small solar zenith angles. Aerosols also play a role in determining the absorption of solar radiation in the atmosphere. The Li et al. [1993] algorithm includes a nominal aerosol with an optical depth of 0.05 at $550 \mathrm{~nm}$, which is inaccurate for heavy aerosol loading or where the aerosol is strongly absorbing. Correction terms accounting for these two important atmospheric influences on the SSNR were implemented in this study.

Input Data

TOA albedo data are from the high-resolution (approximately 4-km pixel-level data) GOES-8 data set. This satellite data set, generated by the NASA Langley Cloud and Radiation Research Group, uses visible and infrared radiances to derive various cloud and radiation products [Minnis and Smith, 1998]. The lack of on-board calibration requires a careful postlaunch calibration, followed by a narrowband-to-broadband conversion, in order to obtain broadband fluxes from the narrowband uncalibrated digital readings [Minnis et al., 2002]. Both data processes are subject to uncertainties [Trishchenko and Li, 1998; Minnis et al., 2002]. As a result, the GOES-8 products have been revised several times. Good accuracy in radiation 
quantities was demonstrated in the later versions as described in Minnis et al. [2002]. Employed here was the data set last modified in March 2004 (ftp://angler.larc.nasa.gov/public/products/armsgp/visst-pixel-bin/goes8/). It encompasses a domain of approximately $400 \times 400 \mathrm{~km}^{2}$ centered on the SGP CF site in north-central Oklahoma and produces output for about every half-hour on a daily basis. Data sets covering the periods of March, May, July, August, September, October, and December of the year 2000 were used in this study. In addition to TOA broadband radiative fluxes, the data set provides cloud macrophysical properties such as cloud-top height and pixel cloudiness and cloud microphysical properties such as effective radius.

Another input parameter to the models is precipitable water (PW) and this quantity was interpolated from the $2.5^{\circ} \times 2.5^{\circ}$ global National Centers for Environmental Prediction (NCEP) reanalysis [Kalnay et al., 1996]. This data set covers the period from 1948 to the present and each file contains data for one year with values of precipitable water given at four times on each day $(0,6,12,18$ UTC). For the simulations performed here, the subset of data encompassing the SGP region for the year 2000 was extracted first. Bilinear interpolation was performed to determine PW at the GOES data grids of high resolution from the NCEP PW reanalysis data of coarser resolution. A more extensive PW data set is available from the SGP where five-minute averages of microwave radiometer (MWR) retrievals have been made on a continual daily basis since about 1994 [Liljegren and Lesht, 1996] at the CF and four extended facilities located at the perimeter of the SGP domain. Although these data are more accurate, they do not have the spatial coverage needed to determine the PW over the entire SGP domain. Another source of PW data is from the network of Global Positioning System stations located within and around the SGP domain [Rocken et al., 1995]. This data has a better coverage than the MWR data but is still 
inadequate. For the year 2000, for example, many days of data are missing. To evaluate uncertainties in the SSNR due to PW, we derived the SSNR using PW data from the NCEP reanalysis and the SSNR using the MWR PW data. For March 2000 at domain sizes of $4 \times 4 \mathrm{~km}^{2}$ and $400 \mathrm{x} 400 \mathrm{~km}^{2}$ (where the CF value for PW was used for all pixels), the standard deviations between the two retrievals are small $\left(2.2 \mathrm{Wm}^{-2}\right.$ and $2.8 \mathrm{Wm}^{-2}$, respectively).

Aerosol optical depths at $550 \mathrm{~nm}$ were derived from Aerosol Robotic Network atmospheric extinction measurements taken at wavelengths of 340, 380, 440, 500, 670, 870 and $1020 \mathrm{~nm}$ at the CF [Holben et al., 2001]. Data from 1994 until September 2001 were used to derive the Ångstrom exponent from which the aerosol optical depths at $550 \mathrm{~nm}$ were determined. Its mean value is 0.138 for the data employed. The data at the CF were assumed to be representative of the entire SGP domain, given that aerosol loading is not expected to vary greatly over this generally rural area. Exceptions can occur during the spring season when

localized increases in aerosol load can occur due to the agricultural practice of burning fields in preparation for reseeding.

\section{Methodology}

Unlike ground-based point measurements, satellite-measured radiances represent areal means over the footprints of the satellite pixels. Further averaging the pixel-level data, one may obtain mean fluxes over spatial domains of any size. Therefore, through use of high-resolution satellite data, one can gain further insight into the statistics of the spatial variability of the radiation field. Also, frequent observations, such as those provided by a geostationary satellite, facilitate the examination of the temporal variability of the radiation field. 
To that end, we calculated areal means of SSNR over spatial domains of different size and different temporal intervals. The number of pixels within a domain can range from a single pixel to several thousand pixels. The model of $\mathrm{Li}$ et al. [1993] was first applied to each individual pixel and the correction factors accounting for the effects of aerosols and cloud-top height on atmospheric absorption were added, depending on whether the pixel was identified as clear or cloudy. If the pixel was identified as clear, the aerosol correction factor was added. If the pixel was identified as cloudy, the cloud-top height correction factor was applied. Different domain sizes were selected and all were centered on the CF. The SSNR was computed for every half-hour during the daytime for all the days when satellite data was available. Domain sizes were chosen to be representative of typical scales used in various SCM and GCM modeling schemes and range from $10 \times 10 \mathrm{~km}^{2}$ to $400 \times 400 \mathrm{~km}^{2}(10 \times 10,20 \times 20,50 \times 50,100 \times 100,200 \times 200$, 300x300, and 400x400 $\mathrm{km}^{2}$ ). With the daytime SSNR calculated for each half-hour in a day, temporal averaging on various scales can be done. Averaging intervals chosen in this study were 1, 2, 4, 8 hours, together with daily means as well as 5 and 10-day means, and monthly means.

\section{Results}

\subsection{Validation}

The satellite-retrieved SSNR are first validated against ground-based observations to ensure that the satellite-estimated SSNR can reasonably reproduce surface measurements. From satellite retrievals at half-hour intervals, the mean SSNR was computed over a gridbox with an

area of $4 \times 4 \mathrm{~km}^{2}$ centered on the CF and averaged over one hour. Although the size of the gridbox was selected in a somewhat ad hoc manner, it represents the bulk of the sky area 
exposed to a radiometer. The gridbox mean fluxes were compared to ground measurements averaged over an hour. The observed SSNR was derived from measurements of downwelling surfaces fluxes (corrected for the thermal offset) from the Solar Infrared Radiation Station and measurements of broadband surface albedo from upward and downward pointing radiometers deployed at the CF. To reduce uncertainties resulting from surface albedo, the average value of the measurements made from the $10-\mathrm{m}$ and 60 -m towers were employed; the surfaces below the towers are covered by grass (dry grass in winter) and wheat. Note that surface albedo measurements generally have a very limited spatial coverage, which may introduce significant uncertainties in solar radiative transfer calculations [ $\mathrm{Li}$ et al., 2002]. The mean of the albedo measurements made from the two towers has a better representation of the surface reflective characteristics surrounding the CF [Michalsky et al., 2003].

In Figure 2, comparisons of the SSNR estimated from satellite and surface observations of SSNR are presented. In general, the two sets of data agree fairly well, especially in terms of relative differences. Biases range from $-14 \mathrm{Wm}^{-2}$ to $27.0 \mathrm{Wm}^{-2}$ and the root-mean-square errors (RMSE) range from $30 \mathrm{Wm}^{-2}$ to $48 \mathrm{Wm}^{-2}$ with the smallest biases occurring in March and the smallest RMSE in September. Seasonal changes in surface albedo may play an important role in the biases, while the RMSE is dictated primarily by cloud variability. In September 2000, uniform cloud systems dominate the region [Dong et al., 2000] so there is less variability in the radiation field hence less deviation from the point measurements. To a large extent, the scatter in the plots is caused by the mismatch between satellite estimates and ground measurements in time and space. Satellite estimates were averaged over a $4 \mathrm{x} 4-\mathrm{km}^{2}$ area centered on the CF and they do not necessarily coincide with the exact location of the instrument and so some spatial mismatch is expected. Also, the frequencies of the satellite estimates and the surface observations differ 
significantly. The one-hour averages of satellite-estimated SSNR are calculated from three data points separated by 30 minutes. Observations of downwelling surface fluxes were made at a temporal resolution of 1 minute and surface albedo measurements were made at a temporal resolution of 15 minutes. Thus, the ground data contain more information concerning the temporal variation of the SSNR. On the other hand, satellite estimates better describe the spatial variation of the SSNR.

The above discussion implies that the scatter seen in the comparisons stems from both physical causes and statistical sampling errors. The latter is linked with cloud variability. To gain further insight into the discrepancies related to data sampling, two comparisons are made over a spatial domain of $400 \mathrm{x} 400 \mathrm{~km}^{2}$. Table 1 presents the mean and RMSE differences for the comparisons between the means of satellite retrievals averaged over the entire domain and surface observations made at all radiation stations within this domain. While the biases are generally compatible to those shown in Figure 2, the RMSEs are reduced substantially. The reduced RMSEs, albeit still significant, are attributed almost exclusively to the sampling uncertainties. This is clearly seen from Table 2, which gives the statistics of comparisons between two satellite retrievals. One retrieval is the mean of all pixels falling inside the $400 x 400-$ $\mathrm{km}^{2}$ domain, while the other is the mean of the retrievals over $4 \times 4-\mathrm{km}^{2}$ gridboxes surrounding each radiation station located within the domain. Note that the latter data may be regarded as simulated surface observations. Since the two sets of data were all retrieved from satellite data using the same algorithm, their differences attest to the sampling uncertainties. The fact that the RMSE values are generally similar indicates that the bulk of the differences are caused by sampling errors that are associated with cloud variability. This finding helped in devising a useful tool to investigate observation sampling errors, as described in the following section. 


\subsection{Spatial sampling}

We first investigate sampling uncertainties incurred by using single-point data to represent a gridbox of varying size. This investigation has a general implication since the vast majority of ground observations around the world are made at single locations. Such singlepoint measurements have been widely employed in validating GCMs with gridbox sizes of 200x200 km² [Wild et al., 1995].

Point measurements of SSNR at the CF were simulated and compared with areal means in order to evaluate their representativeness over regions of varying scales typical of model grid cells. Table 3 give the means and RMSEs for the comparisons of satellite-estimated SSNR averaged over a $4 \mathrm{x} 4-\mathrm{km}^{2}$ gridbox surrounding the CF (a proxy for ground observations at the CF) with those retrieved over areas of varying size $(10 \times 10,20 \times 20,50 \times 50,100 \times 100,200 \times 200$ and 400x400 $\mathrm{km}^{2}$ ), typical of grid cells for cloud resolving models up to GCMs. Since the physical causes for any systematic errors are removed from such comparisons, they exhibit little bias and trend. As the gridbox size increases, the RMSE increases dramatically. As the domain size increases, more variability in the SSNR occurs due to changes in cloud conditions. A similar result was shown in Long et al. [2002] using cloud amount data from their Surface Cloud Grid product. A $0.25^{\circ} \times 0.25^{\circ}$ grid of cloud amount over the ARM SGP area was generated and from comparisons of the cloud amount at the CF with cloud amount over gridboxes of increasing size, they found that the CF data became less representative of the larger area. Note that the RMSE values represent the inherent uncertainties of ground-based observations due to spatial sampling. As a result, one may not expect, nor attempt to reduce, model-observation differences to within 
this range. For example, the results presented in Table 1 for March 2000 imply that the best accuracy one may achieve with a model of $10 \times 10-\mathrm{km}^{2}$ resolution would be $16 \mathrm{Wm}^{-2}$, which increases to $46 \mathrm{Wm}^{-2}$ and $64 \mathrm{Wm}^{-2}$ as the model domain increases to $200 \times 200 \mathrm{~km}^{2}$ and $400 \times 400$ $\mathrm{km}^{2}$, respectively.

In addition to the gridbox size, the RMSE changes with temporal averaging intervals and cloud systems. The latter is echoed partially in the comparisons for different months. Figure 3 shows the RMSE as a function of spatial domain size for different averaging intervals (instantaneous, 1-hour, 4-hour, 1-day, 5-day and 10-day) for March, May, July, September, and December. The plots convey several pieces of information. First, the RMSE varies considerably with month. The SSNR for September and December shows the least variability. For a 400x400$\mathrm{km}^{2}$ gridbox, the RMSE calculated from instantaneous data and data averaged over an hour and 4 hours is less than those for other months by more than $25 \mathrm{Wm}^{-2}$. July is the most variable month, presumably caused by the prevalence of small convective clouds. However, as the averaging interval increases, the difference among different months diminishes. This is because clouds have certain temporal scales. As the averaging interval is prolonged, more clouds are captured. For a one-day averaging interval, the RMSE decreases substantially relative to those averaged over hours but the differences among various months are still significant. Apparently, the frequency of clouds lasting longer than one day varies among the five months. For typical GCM grids of 200x200 $\mathrm{km}^{2}$, the daily sampling error ranges from $16 \mathrm{Wm}^{-2}$ to $28 \mathrm{Wm}^{-2}$ in September and July, respectively. They decrease to $10 \mathrm{Wm}^{-2}$ or less if the model grid is reduced to $10 \times 10$ $\mathrm{km}^{2}$. For the 5-day average, however, the differences among the months almost vanish, indicating that most clouds have lifetimes less than 5 days over the study area. 
Another interesting feature is how the dependence of the RMSE on gridbox size varies with the averaging interval. For averaging intervals less than a day, the dependence follows a log function, which gradually transforms into a linear function for intervals longer than a day. This is probably associated with cloud scales. Small-scale clouds exhibit high-frequency variations that last for relatively short periods of time, whereas large-scale clouds, which influence longer-term averaging, show much slower temporal variation. For intervals of 10 days or longer, this dependence is very weak, as most cloud systems have a lifetime shorter than 10 days.

\subsection{Temporal averaging}

Physical processes in the atmosphere occur at various scales in both space and time domains. Over time, the physical state of the atmospheric system can change substantially over a region so it is important to determine appropriate time scales upon which radiative quantities are averaged so that errors incurred in matching between model estimates and validating measurements are minimized. Decreases in the sampling errors in the SSNR with averaging intervals are shown in Figure 4 for different domain sizes and months. A sharp decrease in error occurs as the averaging interval increases to a day; beyond that, there is a tendency to level off to a stable value of small magnitude.

The domain size and season dictate errors resulting from (a) differences between areal means and point measurements and (b) choice of temporal averaging period. The statistics shown in Figures 4 and 5 provide objective measures of what would be an acceptable error for

climate modeling purposes. For example, in spring and for a domain size of $100 \times 100 \mathrm{~km}^{2}$, 
increasing the averaging period from one hour to two hours reduces the sampling error from 34 $\mathrm{Wm}^{-2}$ to $29 \mathrm{Wm}^{-2}$. During the fall (September) for the same domain size and increase in averaging period, the sampling error reduces from $22 \mathrm{Wm}^{-2}$ to $19 \mathrm{Wm}^{-2}$. So averaging the SSNR is an effective means of minimizing matching errors if the physical process under study is not unduly sensitive to time passages.

If the averaging is done over more than five days, the errors remain steady and very small (less than $10 \mathrm{Wm}^{-2}$ ) over all domain sizes. Overall, the highest magnitudes tend to occur in summer (July) when cloud systems are more complex and variable in both time and space [Lazarus et al., 2000; Dong et al., 2000]. Thus, use of the point measurements for validating a model would result in large uncertainties.

Figure 5 shows the difference between the monthly mean SSNR over different domain sizes and the monthly mean SSNR measured at the CF for the months of March, May, July, September, and December. The magnitude of the sampling errors is less than $10 \mathrm{Wm}^{-2}$ for all months and domain sizes and the magnitude of the error diminishes to less than $3 \mathrm{Wm}^{-2}$ for typical GCM grids of 200x200 $\mathrm{km}^{2}$ or less during the spring and summer seasons. While the magnitude of the errors agrees well with the general requirement of an accuracy of $5 \mathrm{Wm}^{-2}$ for climate studies [Suttles and Ohring, 1986], it would be an unrealistic goal for certain regions/seasons, such as September 2000 over the larger 400x400-km² SGP domain. In this case, the inherent sampling error in monthly mean surface observations is $8 \mathrm{Wm}^{-2}$, exceeding the required $5 \mathrm{Wm}^{-2}$.

\subsection{Multiple ground stations}


Since single-point measurements do not represent well areal means over a large domain, multiple radiation stations distributed over a large domain around the CF may improve the spatial representation of the SSNR. General improvement is expected since more observations capture more of the variability in the SSNR. The question remains as to how much improvement in accuracy is gained as the number of stations increases, and how many stations are really needed to meet certain accuracy requirements. To address these questions, domains of different sizes centered on the CF were selected: 100x100, 200x200, 300x300, and 400x400 km², containing 1, 7, 12, and 21 observation sites, respectively. Diagrams of the domains used and the locations of the observation sites are given in Figure 1. For each particular domain size, satelliteestimated SSNR was calculated over $4 \times 4-\mathrm{km}^{2}$ gridboxes centered on each site (a proxy for actual surface measurements). The mean values averaged over all sites inside a particular domain are compared to the satellite-estimated areal means over the domain. RMSEs were calculated between these satellite-simulated point-specific fluxes and the area mean fluxes for hourly means and daily means in March, July, September, and December. The results are presented in Figure 6. The sampling error is the largest for the $100 \times 100-\mathrm{km}^{2}$ gridbox in which there is only one station (the CF). For other domain sizes, the magnitudes of the RMSE vary significantly with month, from about $6 \mathrm{Wm}^{-2}$ (September) to more than $17 \mathrm{Wm}^{-2}$ (March) for the daily means, and from $10 \mathrm{Wm}^{-2}$ (September) to $30 \mathrm{Wm}^{-2}$ (March) for the hourly means; the December values fall somewhere in-between. The closeness in the magnitude of the RMSE obtained over domains ranging from $200 \times 200 \mathrm{~km}^{2}$ to $400 \times 400 \mathrm{~km}^{2}$ indicates that the density of observation stations is proportional to the area of the model grids.

Similar calculations were also conducted for a fixed domain size of about $200 \times 200 \mathrm{~km}^{2}$ using various combinations of the extended facilities contained within this domain. In addition to 
the $\mathrm{CF}$, the domain encompasses radiation stations located in Ashton, Kansas and Byron, Pawhuska, Ringwood, El Reno and Meeker in the state of Oklahoma. The number of observation stations used ranges from 1 (the CF) to 7 (all facilities). Intermediate combinations of the stations used consist of the CF plus an increasing number of the other stations in no particular order. The standard deviations of the differences between the domain-averaged SSNR and the averages of the simulated surface observations for March 2000 were calculated and plotted as a function of the number of surface sites for instantaneous, 1-hr, 4-hr, 1-day, 5-day and 10-day means for March 2000 (Figure 7). In general, the RMSE decreases as the number of observation sites increases. However, the reduction is more significant when using 2 or 3 sites, after which the RMSE more or less levels off (and sometimes increases a bit due to random sampling). This means that for computing domain mean SSNR, there is no need for an overly dense network of observation stations. The number of stations required is contingent upon the averaging period and season. The shorter the averaging interval, the more stations are needed to reduce the RMSE of the SSNR. Mean SSNR values averaged over one day remain generally stable as the number of stations increases from 1 to 7 . Similar analysis for other months (not shown) reveals a more significant reduction in the magnitude of the RMSE as the number of sites increases.

\section{Summary}

Radiation measurements have been widely employed for evaluating cloud parameterization schemes and model simulation results. As model results are given over grids at different time intervals, different data have been employed to obtain the estimates of area averaged surface solar radiation for time intervals of an hour to a few days. They include (a) 
averages of point measurements, (b) satellite-based estimates, and (c) a combination of the two, in which the point measurements are used to correct biases in the satellite estimates.

In this study, we take advantage of the high spatial and temporal resolution of a recently processed Geostationary Operational Environmental Satellite data set of cloud properties to mimic ground-based measurements of surface solar net radiation (SSNR) of varying spatial density and temporal frequency and characterize their observation uncertainties caused by cloud variability at different scales in different seasons. Such scale-dependent statistics of observation uncertainties provide critical constraints on model-observation comparisons, and are thus valuable for improving and validating cloud parameterization schemes. The Department of Energy Atmospheric Radiation Measurement program’s observation network in Oklahoma and Kansas provided a useful testbed for this study. In terms of spatial averaging, a single observation site (the Central Facility) does an increasingly poor job of representing areal means of SSNR as the domain size increases. Averaging the surface net radiation at more observation sites results in a decrease in error as the domain size (and number of observation sites) increases. As for temporal averaging, increasing the time interval leads to a general decrease in error for all domain sizes and all seasons. Instantaneous measurements incur the greatest error. Averaging over periods greater than 5 days smoothes out the error to a generally stable magnitude of less than $15 \mathrm{Wm}^{-2}$ over all domain sizes. Some ramifications for climate and cloud modeling studies are that for fine-scale models with short integration intervals, such as cloud-resolving models, point measurements do not provide the best representation of radiative quantities. For large-scale models such as GCMs, the use of multiple observation sites does a reasonable job at capturing the SSNR field over a large domain. When modeled radiation quantities are compared against ground observations, the inherent uncertainties due to sampling errors must be taken into 
consideration. Such inherent uncertainties are simulated from our satellite retrievals, which are given as functions of model domain size, averaging period, number of observation stations, and month. If the difference between modeled and observed radiation quantities is comparable to or less than the corresponding inherent uncertainty, no further insight may be gained with regard to the model's deficiencies. Such statistics are thus valuable for validating models when testing their parameterization schemes.

With regard to the three methods of obtaining area averaged SSNR data, it is fair to say that ground observations have the highest accuracy and frequency, while satellite estimates provide the most extensive spatial coverage and uniform quality. A combination of the two sets of data is recommended for model evaluation. Not only are the ground observations useful for validating satellite retrievals, they may also be used to remove biases caused by infrequent sampling, especially for regions outside the coverage of the geostationary satellites.

\section{Acknowledgements}

The Cloud and Radiation group led by Pat Minnis at the NASA Langley Research Center generated the GOES products used in the study. NCEP Reanalysis data were obtained from the website of the NOAA-CIRES Climate Diagnostics Center in Boulder, Colorado (http://www.cdc.noaa.gov/). Aerosol data were downloaded from the website of the Aerosol Robotic Network (http://aeronet.gsfc.nasa.gov/). This study has been supported by the DOE ARM grants DE-FG02-01ER63166 and DE-FG02-02ER63351. 


\section{References}

Ackerman, T., and G. Stokes (2003), The Atmospheric Radiation Measurement Program, Physics Today, 56, 38-45.

Barnett, T.P., J. Ritchie, J. Foat, and G. Stokes (1998), On the space-time scales of the surface solar radiation field, J. Clim., 11, 88-96.

Barnett, T.P., J. Ritchie, J. Foat, and G. Stokes (1996), Relating Atmospheric Radiation Measurement observations to general circulation scales, Sixth Atmospheric Radiation Measurement Science Team Meeting, San Antonio, Texas.

Browning, K.A., and coauthors (1993), GEWEX cloud system study (GCSS), Bull. Amer. Meteor. Soc., 74, 387-399.

Conant, W.C., V. Ramanathan, F.P.J. Valero, and J. Meywerk (1997), An examination of the clear-sky solar absorption over the central equatorial Pacific: Observation versus models, J. Clim., 10, 1874-1884.

Dong, X., P.Minnis, T.P.Ackerman, E.Clothiaux, G.Mace, C.Long, and J.Liljegren (2000), A 25month database of stratus cloud properties generated from ground-based measurements at the Atmospheric Radiation Measurement Southern Great Plains Site, J. Geophys. Res., 105, 4529-4537.

Ellingson, R.G.(2000), The ARM radiative flux (IRF) working group in 2000: A summary of accomplishment, strengths, weaknesses, and ideas for future activities, ARM Vision 2000 Report, pp. 15.

Feng J., and H. G. Leighton (2003), Effects of absorbing aerosols on the determination of the surface solar radiation, J. Geophys. Res., 108 (D7), 4227, doi:10.1029/2002JD002852. 
Jing, X., and R. Cess (1998), Comparison of atmospheric clear-sky radiation models to collocated satellite surface measurements in Canada, J. Geophys. Res., 103, 28,81728,824 .

Holben, B.N., D. Tanré, A. Smirnov, T.F. Eck, I. Slutsker, N. Abuhassan, W.W. Newcomb, J.S. Schafer, B. Chatenet, F. Lavenu, Y.J. Kaufman, J. Vande Castle, A. Setzer, B. Markham, D. Clark, R. Frouin, R. Halthore, A. Kareli, N.T. O’Neill, C. Pietras, R.T. Pinker, K. Voss, and G. Zibordi (2001), An emerging ground-based aerosol climatology: Aerosol optical depth from AERONET, J. Geophys. Res., 106, 12067-12097.

Kalnay, E., and coauthors (1996), The NCEP/NCAR reanalysis 40-year project, Bull. Amer. Meteor. Soc., 77, 437-471.

Krueger, S. K., and J. E. Burks (1998), Radiative fluxes and heating rates during TOGA COARE over the Intensive Flux Array. Proceedings of the Eighth Atmospheric Radiation Measurement (ARM) Science Team Meeting, Tucson, Arizona , DOE, 399-402.

Krueger, S. K., and S. M. Lazarus (2000), Intercomparison of multi-day simulations of convection during TOGA COARE with several cloud resolving and single-column models. Proceedings of the Workshop on Cloud Processes and Cloud Feedbacks in Large-Scale Models, ECMWF, Reading, Berkshire, UK, 9-13 November 1998,WCRP110, [WMO/TD No. 993], 84-91.

Lazarus, S.M., S.K. Krueger, and G.G. Mace (2000), A cloud climatology of the Southern Great Plains ARM CART, J. Climate, 13, 1762-1775.

Li, Z. (1998), Influence of absorbing aerosols on the inference of solar surface radiation budget and cloud absorption, J. Clim., 11, 5-17. 
Li, Z. and H.G.Leighton (1993), Global climatologies of solar radiation budgets at the surface and in the atmosphere from 5 years of ERBE data, J. Geophys. Res., 98, 4919-4930.

Li, Z., and L. Moreau (1996), Alteration of atmospheric solar absorption by clouds: Simulation and observation, J. Appl. Meteor., 35, 653-670.

Li, Z., M. C. Cribb, and A. P. Trishchenko (2002), Impact of surface inhomogeneity on solar radiative transfer under overcast conditions, J. Geophys. Res., 107(D16), 4294, doi:10.1029/2001JD000976.

Li, Z., H.G.Leighton, K.Masuda, and T.Takashima (1993), Estimation of SW flux absorbed at the surface from TOA reflected flux, J. Clim., 6, 317-330.

Liljegren, J.C., and B.M. Lesht (1996), Measurements of integrated water vapor and cloud liquid water from microwave radiometers at the DOE ARM Cloud and Radiation Testbed in the U.S. Southern Great Plains, presented at the IEEE International Geosciences and Remote Sensing Symposium, Lincoln, Nebraska.

Long, C.N., T.P. Ackerman, and J.E. Christy (2002), Variability across the ARM SGP area by temporal and spatial scale, Twelfth Atmospheric Radiation Measurement Science Team Meeting, St. Petersburg, Florida.

Masuda, K., H.G. Leighton, and Z. Li (1995), A new parameterization for the determination of solar flux absorbed at the surface from satellite measurements, J. Clim., 8, 1615-1629.

Michalsky, J, Q. Min, J. Barnard, R. Marchand, and P. Pilewskie (2003), Simultaneous spectral albedo measurements near the Atmospheric Radiation Measurement Southern Great Plains (ARM SGP) central facility, J. Geophys. Res., 108(D8), 4254, doi: 10.1029/2002JD002906. 
Minnis, P., and W.L. Smith (1998), Cloud and radiative fields derived from GOES 8 during SUCCESS and the ARM-UAV spring, Geophys. Res. Lett., 25,1113.

Minnis, P., L. Nguyen, D.R. Doelling, D.F. Young, W.F. Miller, and D.P. Kratz (2002), Rapid calibration of operational and research meteorological satellite imagers: Part I: Evaluation of research satellite visible channels as references, J. Atmos. Oceanic Tech., 19, 12331249.

Morcrette, J.-J.(2002), Assessment of the ECMWF model cloudiness and surface radiation fields at the ARM SGP site, Mon. Wea. Rev., 130, 257-277.

Perez, R., R. Seals and A. Zelenka (1997), Comparing satellite remote sensing and ground network measurements for the production of site/time specific irradiance data. Solar Energy, 60, 89-96.

Ramanathan, V., B. Subasilar, G.J. Zhang, W. Conant, R.D. Cess, J.T. Kiehl, H. Grassl, and L. Shi (1995), Warm pool heat budget and shortwave cloud forcing: A missing physics, Science, 267, 499-503.

Randall, D., S. Krueger, T. Del Genio, S. Ghan, M. Zhang, and R. Cederwall (2000), ARM cloud parameterization and modeling working group, ARM Vision 2000 Report, pp.16.

Randall, D. and Co-authors (2003), Confronting models with data: The GEWEX cloud system study, Bull. Am. Meteorol. Soc., 84, 455-469.

Rocken, C., T. Van Hove, J. Johnson, F. Solheim, R. H. Ware, M. Bevis, S. Businger, S. Chiswell (1995), GPS/STORM - GPS Sensing of Atmospheric Water Vapor for Meteorology, J. Atmos. Oceanic Technol., 12, 468-478.

Rossow, W.B., C. Delo, and B. Cairns (2002), Implications of the observed mesoscale variations of clouds for the Earth's radiation budget. J. Clim., 15, 557, doi:10.1175/1520-0442. 
Stokes, G.M., and S.E. Schwartz (1994), The Atmospheric Radiation Measurement (ARM) program: Programmatic background and design of the cloud and radiation test bed, Bull. Amer. Meteor. Soc., 75,1201-1221.

Suttles, J.T., and G. Ohring (1986), Report of the workshop on surface radiation budget for climate applications. Technical Report WCP-115, World Meteorological Organization.

Trishchenko, A., and Z. Li (1998), Use of ScaRaB measurements for validating a GOES-based TOA radiation product, J. Appl. Meteor., 37, 591-605.

Waliser, D. E., W. D. Collins, and S. P. Anderson (1996), An estimate of the surface shortwave cloud forcing over the western Pacific during TOGA COARE, Geophys. Res. Lett., 23, 519-522.

Waliser, D.E., R.A. Weller, and R.D. Cess (1999), Comparisons between buoy-observed, satellite-derived, and modeled surface shortwave flux over the subtropical North Atlantic during the Subduction Experiment, J. Geophys. Res., 104, 31301-31320.

Wielicki, B.A., and Coauthors (1996), Clouds and the Earth’s Radiant Energy System (CERES): an Earth Observing System experiment. Bull. Amer. Meteor. Soc., 77, 853-868.

Wild, M., A. Ohmura, H. Gilgen, and E. Roeckner (1995), Validation of general circulation model radiative fluxes using surface observations, J. Clim., 8, 1309-1324. 
Table 1. The mean and RMSE differences (in $\mathrm{Wm}^{-2}$ ) for comparisons between the surface solar net radiation estimated from all pixels over the $400 \times 400 \mathrm{~km}^{2}$ domain and that observed at 21 ground stations of the extended facilities.

\begin{tabular}{ccc}
\hline Month & RMSE & Mean Difference \\
\hline March & 23.9 & -4.9 \\
July & 24.2 & 30.7 \\
September & 19.3 & 21.3 \\
December & 34.4 & -19.0 \\
\hline
\end{tabular}

Table 2. Same as Table 1 except that values of the ground observation are replaced by satellite retrievals over $214 \times 4 \mathrm{~km}^{2}$ areas surrounding the ground stations.

\begin{tabular}{crc}
\hline Month & RMSE & Mean Difference \\
\hline March & 28.3 & -8.0 \\
July & 24.0 & -3.7 \\
September & 10.6 & 1.3 \\
December & 15.7 & -8.3 \\
\hline
\end{tabular}

Table 3. The RMSE and mean differences (in $\mathrm{Wm}^{-2}$ ) from comparisons of surface solar net radiation estimated at the CF (averaged over a $4 \mathrm{x} 4-\mathrm{km}^{2}$ gridbox) and over larger domains of varying size from $10 \times 10 \mathrm{~km}^{2}$ to $400 \times 400 \mathrm{~km}^{2}$ for March 2000. The surface solar net radiation was averaged over an hour.

\begin{tabular}{ccc}
\hline Domain Size $\left(\mathrm{km}^{2}\right)$ & RMSE & Mean Difference \\
\hline 10x10 & 16.2 & -0.4 \\
20x20 & 19.8 & 0.9 \\
$50 \times 50$ & 27.4 & 0.1 \\
100x100 & 34.4 & -0.7 \\
200x200 & 46.5 & 0.6 \\
$400 \times 400$ & 64.2 & 1.6 \\
\hline
\end{tabular}




\section{Figure Captions}

Figure 1. Distribution of the ARM extended facilities (left panel) and different model grid cells used in the study $\left(100 \times 100 \mathrm{~km}^{2}, \sim 200 \times 200 \mathrm{~km}^{2}, 300 \times 300 \mathrm{~km}^{2}\right.$ and $400 \times 400 \mathrm{~km}^{2}$, right panel).

Figure 2. Comparisons of surface solar net radiation observed at the CF and estimated from satellite (averaged over a $4 \mathrm{x} 4-\mathrm{km}^{2}$ gridbox centered on the $\mathrm{CF}$ ) for the months of March, July, September and December.

Figure 3. Standard deviation as a function of domain size for the SSNR differences between satellite-simulated "point measurements” and areal mean values over domains of varying size up to $400 \times 400 \mathrm{~km}^{2}$. The comparisons are for instantaneous values and temporally averaged values over different intervals in March (stars), May (squares), July (diamonds), September (triangles), and December (circles).

Figure 4. Similar to Figure 3 but plotted as a function of the averaging period (1-hr, 2-hr, 4-hr, 8-hr, day, 5-day,10-day) for different domains and months.

Figure 5. Difference between monthly mean SSNR simulated for “point measurements” and areal mean values as a function of domain size varying from $10 \times 10 \mathrm{~km}^{2}$ to $400 \times 400$ $\mathrm{km}^{2}$ for March (stars), May (squares), July (diamonds), September (triangles), and December (circles).

Figure 6. The standard deviations of the SSNR differences between satellite-estimated areal means and simulated point-specific values averaged over all stations falling within domains of 100x100, 200x200, 300x300, and 400x400-km² $(1,4,7$, and 21 sites for the 100x100, 200x200, 300x300, and 400x400- $\mathrm{km}^{2}$ domains, respectively) for hourly and daily-averaged values. 
Figure 7. The standard deviation of the differences between domain-averaged SSNR and the means of simulated surface observations at 1-7 stations falling within the domain as a function of the number of stations within a $\sim 200 x 200-\mathrm{km}^{2}$ grid. 

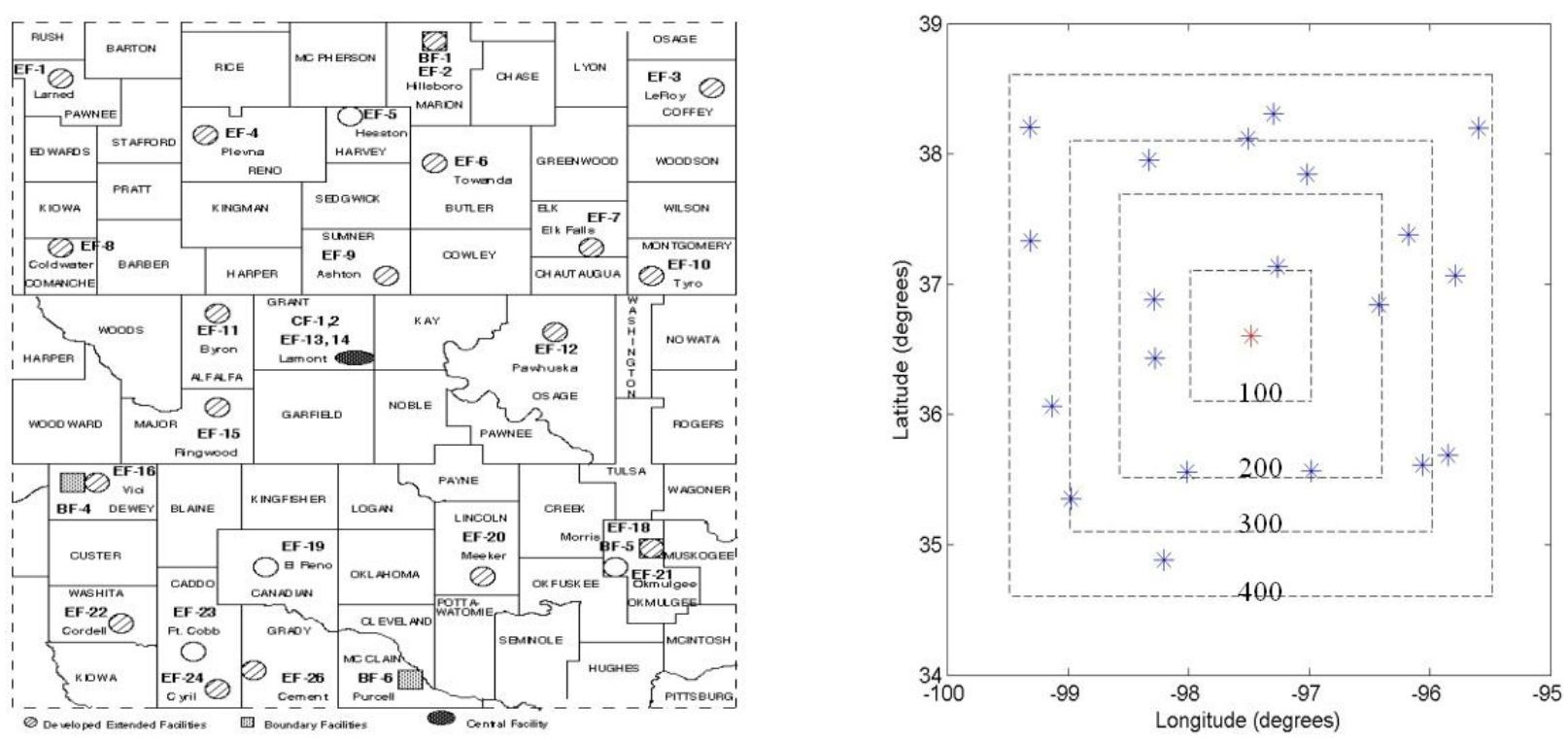

Figure 1. Distribution of the ARM extended facilities (left panel) and different model grid cells used in the study $\left(100 \times 100 \mathrm{~km}^{2}, \sim 200 \times 200 \mathrm{~km}^{2}, 300 \times 300 \mathrm{~km}^{2}\right.$ and 400x400 km², right panel). 

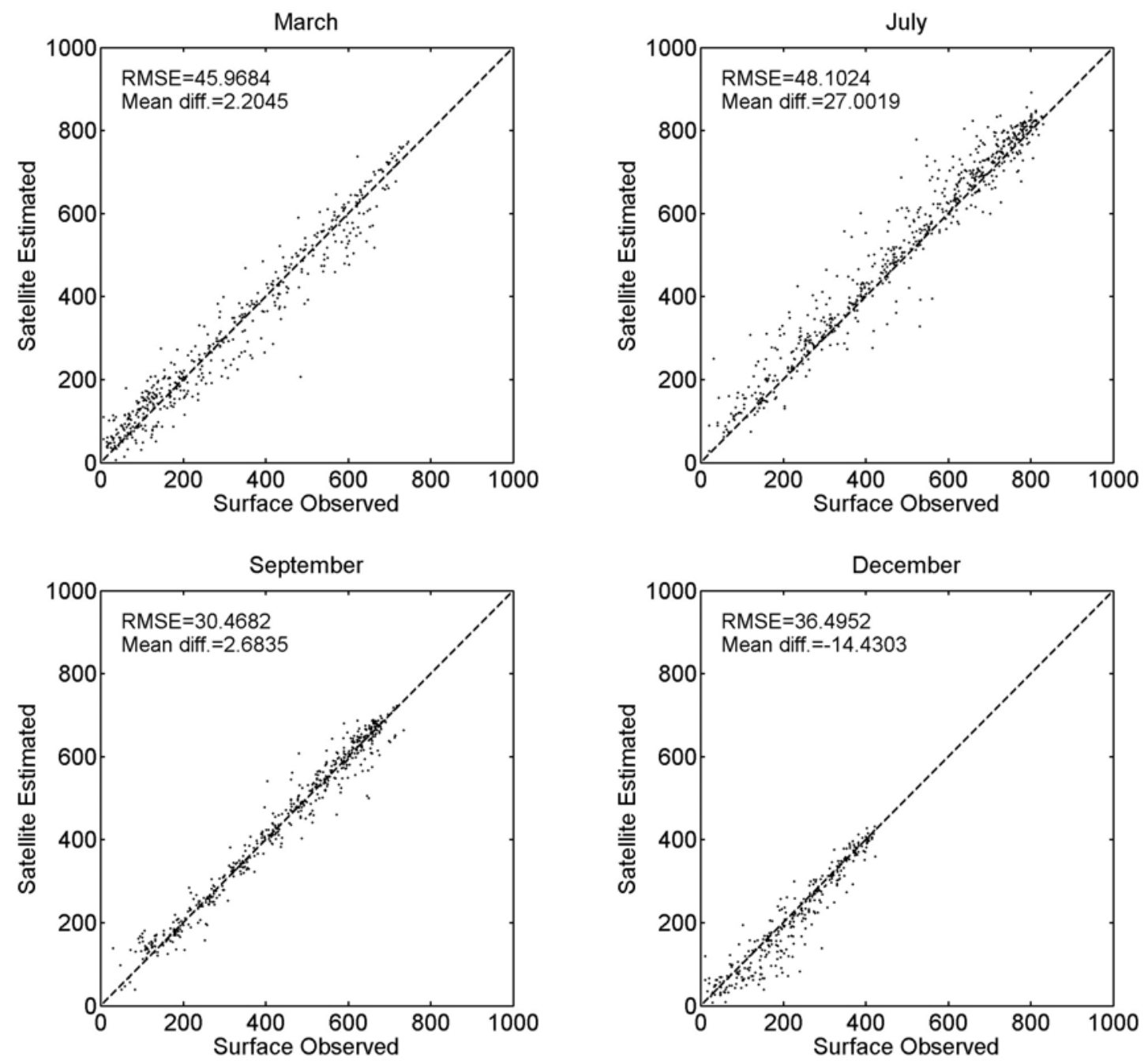

Figure 2. Comparisons of surface solar net radiation observed at the CF and estimated from satellite (averaged over a $4 \mathrm{x} 4-\mathrm{km}^{2}$ gridbox centered on the CF) for the months of March, July, September, and December. 

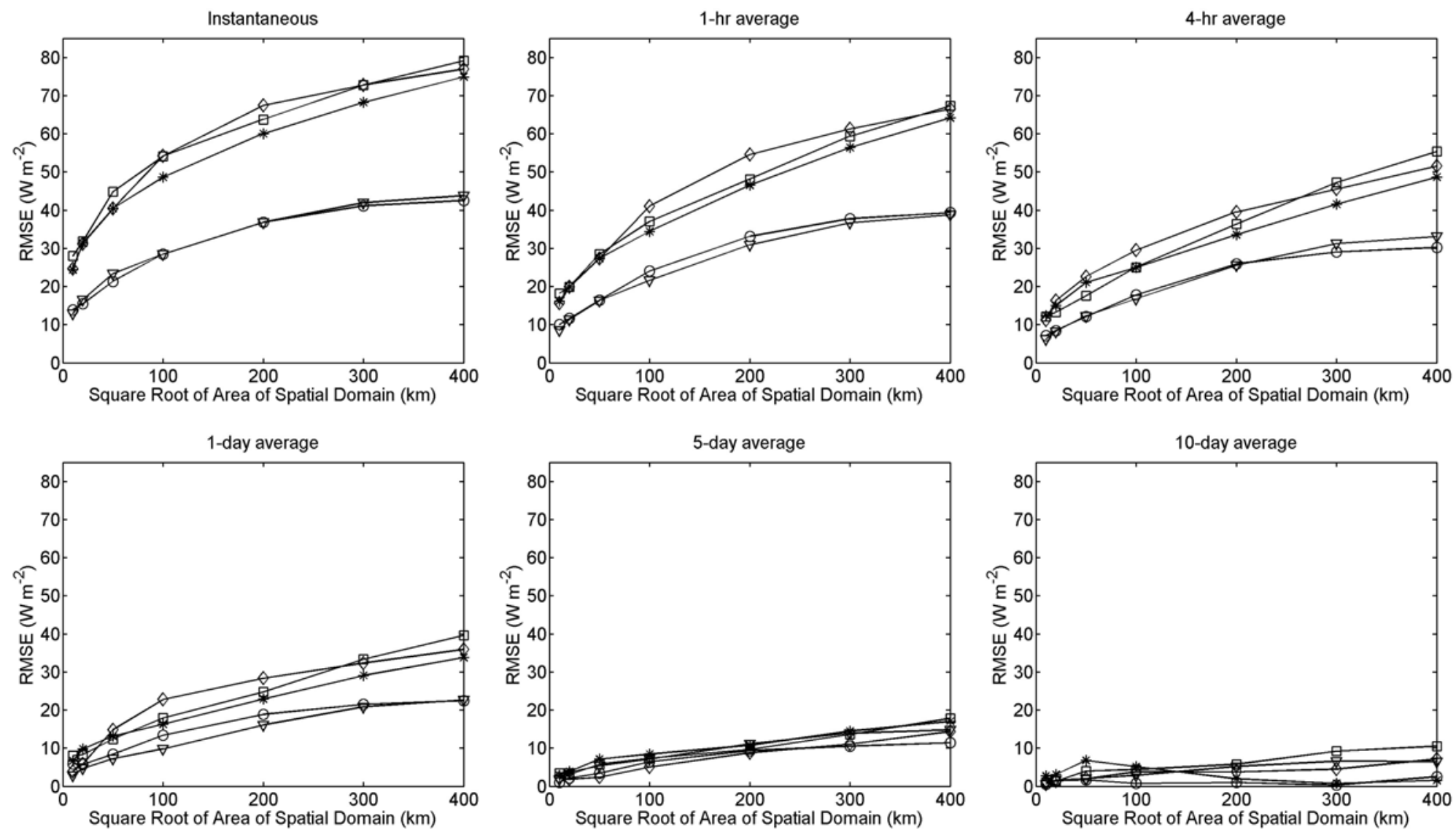

Figure 3. Standard deviation as a function of domain size for the SSNR differences between satellite-simulated "point measurements" and areal mean values over domains of varying size up to $400 \times 400 \mathrm{~km}^{2}$. The comparisons are for instantaneous values and temporally averaged values over different intervals in March (stars), May (squares), July (diamonds), September (triangles), and December (circles). 

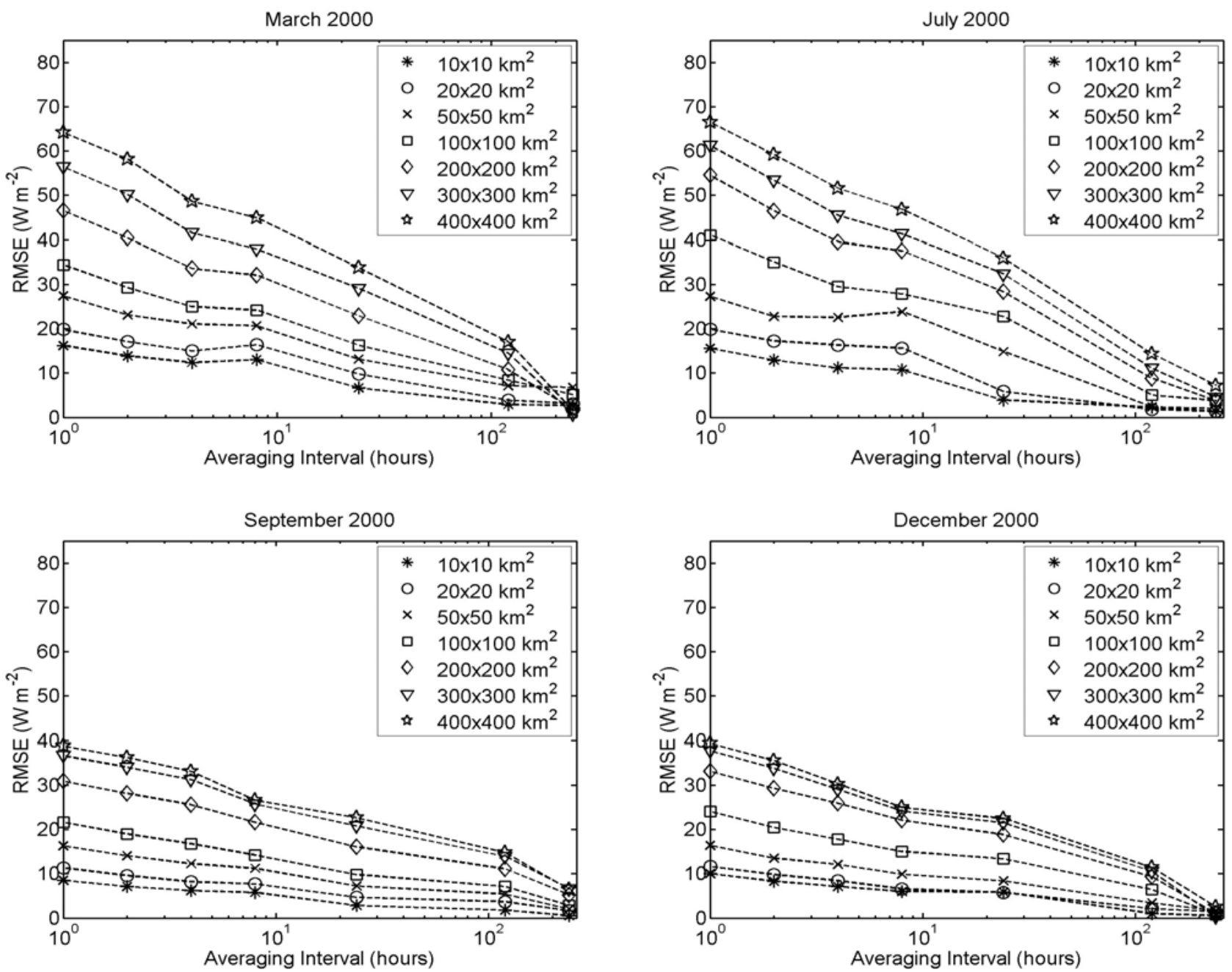

Figure 4. Similar to Figure 3 but plotted as a function of the averaging period (1-hr, 2-hr, 4-hr, 8-hr, day, 5-day, 10-day) for different domains and months. 


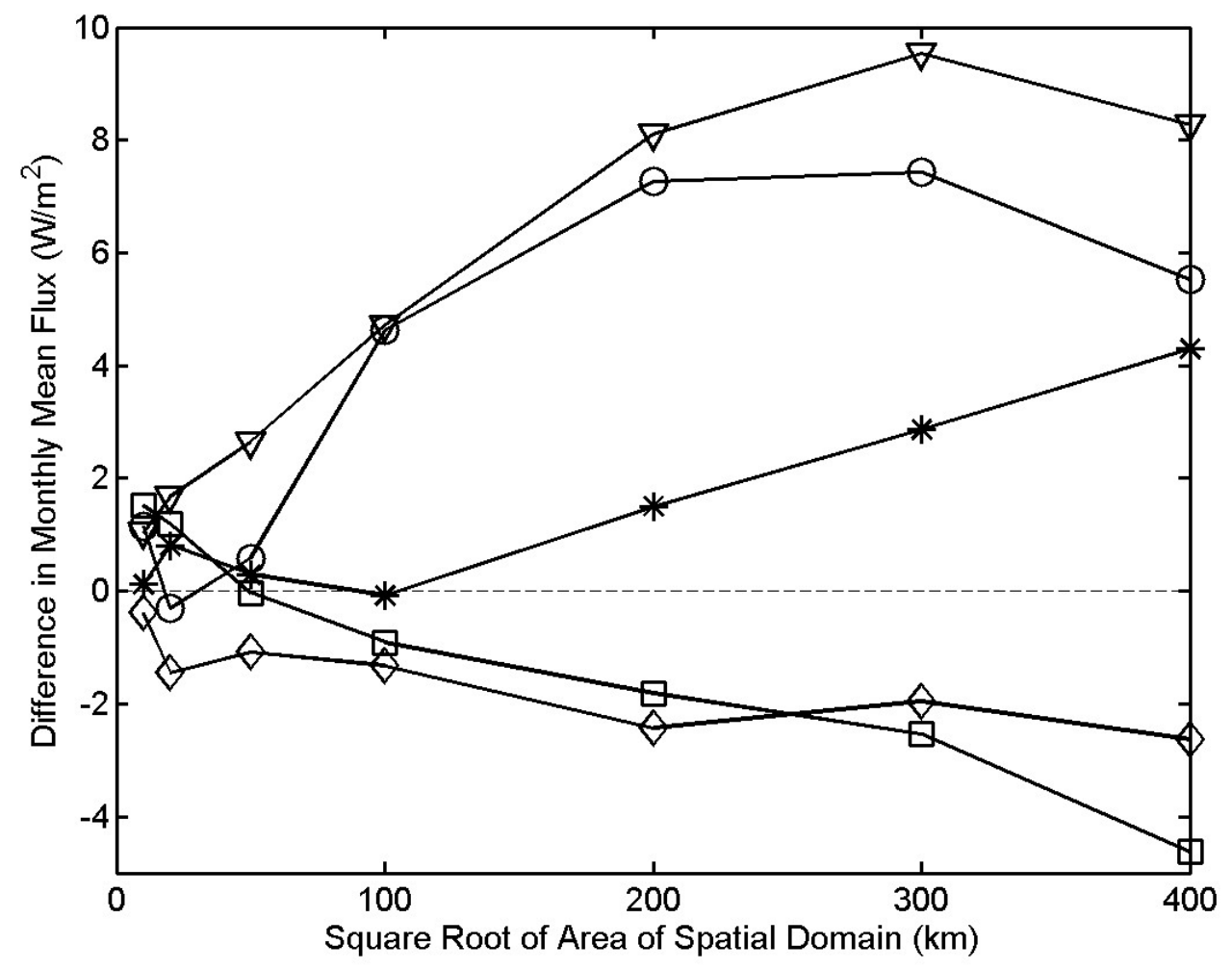

Figure 5. Difference between monthly mean SSNR simulated for "point measurements" and areal mean values as a function of domain size varying from $10 \times 10 \mathrm{~km}^{2}$ to $400 \times 400$ $\mathrm{km}^{2}$ for March (stars), May (squares), July (diamonds), September (triangles), and December (circles). 

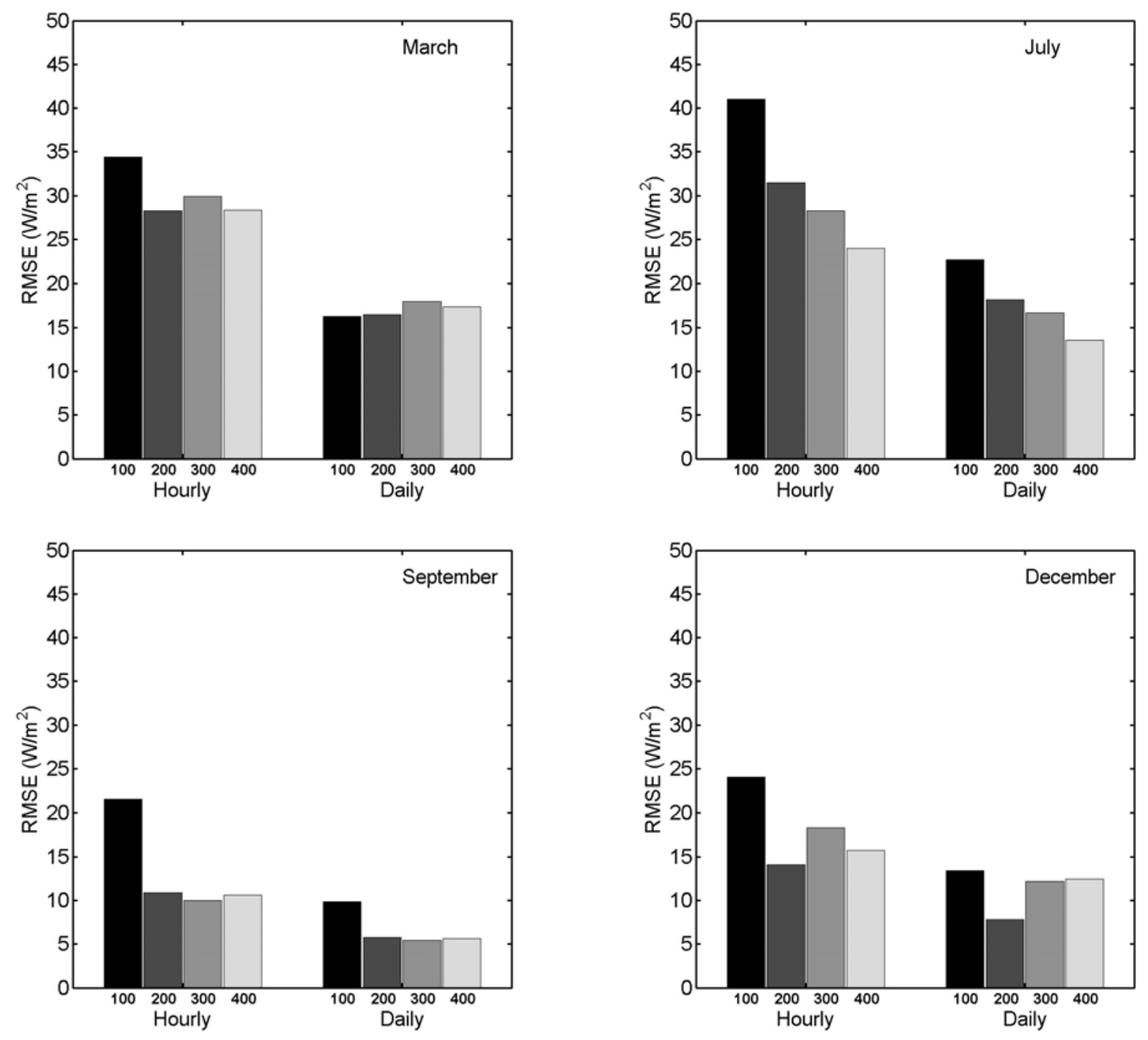

Figure 6. The standard deviations of the SSNR differences between satellite-estimated areal means and simulated point-specific values averaged over all stations falling within domains of 100x100, 200x200, 300x300, and 400x400-km² (1, 7, 12, and 21 sites for the 100x100, 200x200, 300x300, and 400x400-km² domains, respectively) for hourly and daily-averaged values. 


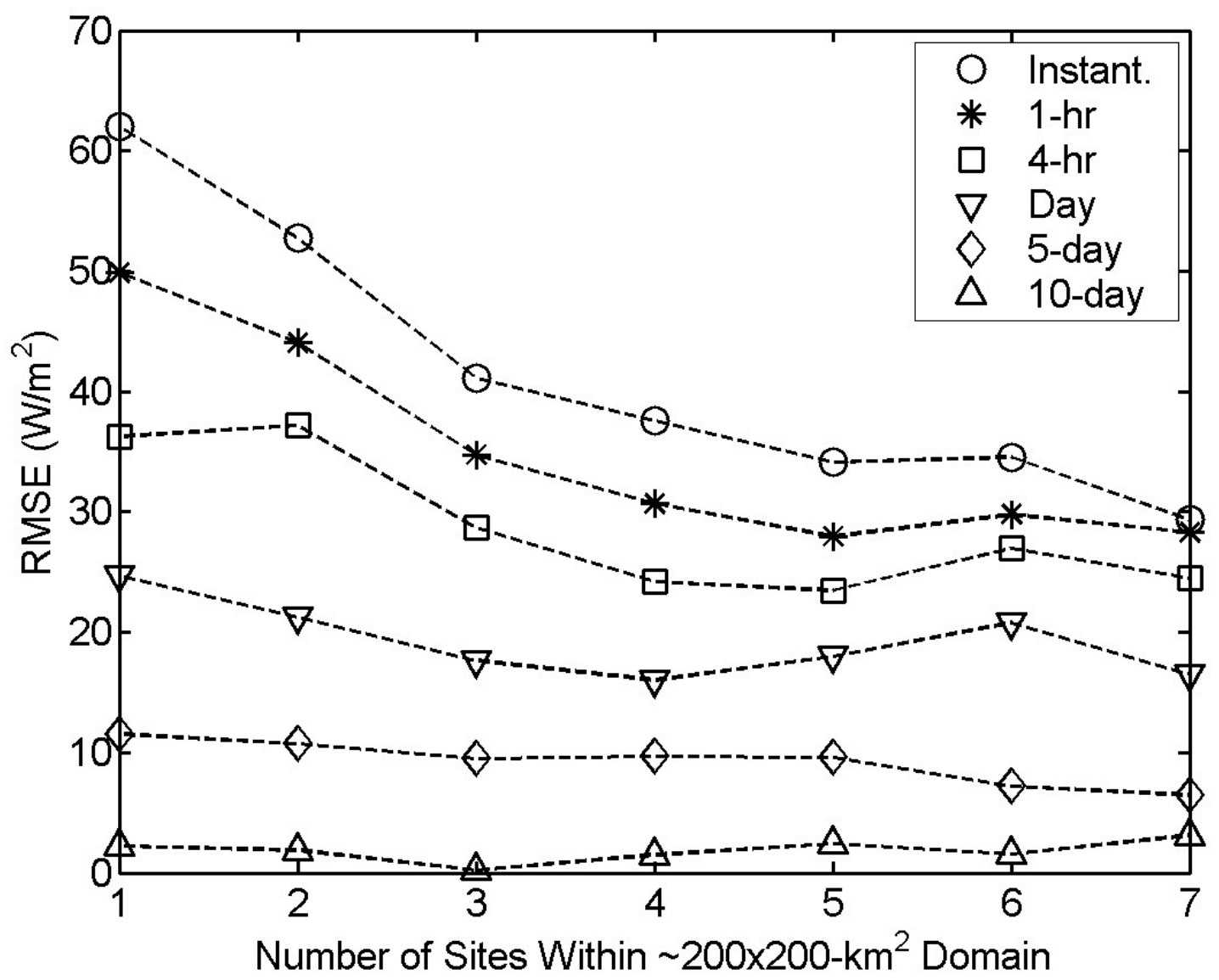

Figure 7. The standard deviation of the differences between domain-averaged SSNR and the means of simulated surface observations at 1-7 stations falling within the domain as a function of the number of stations within a 200 x $200 \mathrm{~km}^{2}$ grid for March 2000. 


\title{
Trends and uncertainties in thermal calibration of AVHRR radiometers onboard NOAA-9 to -16
}

\author{
Alexander P. Trishchenko*(1) , Gunar Fedosejevs ${ }^{(1)}$, Zhanqing Li ${ }^{(2)}$, Josef Cihlar ${ }^{(1)}$ \\ (1) Canada Centre for Remote Sensing, Natural Resources Canada, \\ Ottawa, Ontario, K1A 0Y7 Canada \\ (2) University of Maryland, ESSIC, College Park, MD, 20742, USA \\ * Corresponding author: \\ 588 Booth Street, Ottawa, Ontario, K1A 0Y7, Canada \\ Phone: (613) 9955787 \\ Fax: (613) 9471383 \\ e-mail: trichtch@ccrs.nrcan.gc.ca
}

Submitted to

Journal of Geophysical Research. Atmospheres.

March 21, 2002

Revised August 12, 2002 


\begin{abstract}
Satellite measurements from the infrared (IR) channels of AVHRR/NOAA have been used to derive many important atmospheric, cloud and surface parameters for weather prediction, climate modelling and a variety of environmental studies. Calibration accuracy of the satellite data directly affects accuracies of the derived parameters. So far, very limited attention has been given to the calibration uncertainties of the IR channels. In this study, we analysed the calibration data of AVHRR radiometers onboard polar orbiting satellites NOAA-9 to NOAA-16. We utilized Global Area Coverage (GAC) data, approximately one orbit per month throughout the lifetime of the instruments, available from the NOAA Satellite Active Archive (SAA)
\end{abstract}

AVHRR IR channels 3B, 4 and 5 are calibrated in-flight. Calibration coefficients are derived from measurements of radiance emitted from an internal calibration target (ICT) and deep-space (SP). The overall budget of uncertainties has been evaluated using an in-flight calibration system that includes four thermal platinum resistance thermometers (PRTs) to monitor the ICT temperature. The measurement noise (NE $\Delta \mathrm{T}$ ) was found to vary from $0.03 \mathrm{~K}$ to $0.3 \mathrm{~K}$ at $300 \mathrm{~K}$ depending on the channel and radiometer, and it increases significantly as temperature decreases. Systematic degradation of the radiometric sensitivity of the IR detectors was observed during the lifetime of a radiometer, although the annual rate of degradation is rather small (typically below $1 \%$ per year). A significant correlation between the calibration gain and temperature of a radiometer is often observed. The degradation of a sensor's radiometric sensitivity reduces the radiometric resolution of the AVHRR measurements and expands the upper limit of the measured brightness temperature. 
PRT measurements are subject to significant orbital variation (up to $7 \mathrm{~K}$ ) and inconsistency for some AVHRR radiometers. The inconsistency was especially large for the AVHRR onboard NOAA-12 (up to $4 \mathrm{~K}$ ) and NOAA-14 (up to $3 \mathrm{~K}$ ), but it is less than $0.5 \mathrm{~K}$ for NOAA-15 and -16 . The inconsistency may signify the presence of a thermal gradient across the ICT. Some systematic differences between PRT measurements may also indicate inaccurate characterization of the PRT sensors, for example for AVHRR/NOAA-11 and -14.

The impact of the varying thermal state of the AVHRR environment on the accuracy of AVHRR in-flight thermal calibration was assessed. We found this impact to be significant (up to $0.5 \mathrm{~K}$ and more), and proposed a physical model to explain it. We recommend this model for AVHRR operational in-flight calibration, especially during solar radiative contamination events. Estimates of the PRT thermal response time constant were derived and found to vary between 0.5 and 1.5 minutes among AVHRR radiometers. Overall, we found somewhat higher uncertainties in AVHRR thermal measurements than were assumed previously. 


\section{Introduction}

The Advanced Very High Resolution Radiometer (AVHRR) onboard the National Oceanic and Atmospheric Administration's (NOAA) polar orbiting satellites is perhaps the most widely used sensor for operational and long-term monitoring of global atmospheric, oceanic and terrestrial environments from space [Cracknell, 1997; Rao et al., 1990; Cihlar et al., 2002]. Many important parameters assimilated in numerical weather prediction models employed in climate and other studies are extracted from the AVHRR thermal data. As such, thorough understanding of uncertainties in the thermal calibration data is of critical importance. So far, however, this issue has been poorly addressed [Kidwell, 1998].

The first AVHRR models launched on board Tiros-N, NOAA-6, -8, -10 had four spectral channels. The second model known as AVHRR/2 was deployed on NOAA-7, -9, 11, -12, -14. The AVHRR/2 radiometers had 5 spectral channels: 1) visible $0.55-0.68 \mu \mathrm{m}, 2$ ) near-infrared (NIR) channel 0.725-1.10 $\mu \mathrm{m}, 3$ ) shortwave infrared (channel 3B SWIR) 3.55$3.93 \mu \mathrm{m}$, 4) thermal infrared (IR) $10.3-11.3 \mu \mathrm{m}$, and 5) thermal infrared $11.5-12.5 \mu \mathrm{m}$. New AVHRR/3 instruments onboard NOAA-15 and -16 have an additional channel 3A in the 1.585-1.635 $\mu \mathrm{m}$ spectral region. Channels $3 \mathrm{~A}$ and 3B operate interchangeably during the day (3A, ascending orbits) and night (3B, descending orbits), respectively.

Data from the AVHRR visible and NIR channels have been used to determine different land surface parameters such as surface reflectance (Trishchenko et al., 2002), land cover types [Cihlar, 2000], normalised difference vegetation index (NDVI) [Kidwell, 1990] and absorbed photosynthetically active radiation (APAR) [Li et al., 1997], and to retrieve aerosol optical depth [Ignatov and Stowe, 2002 a, b; Stowe et al., 1997], among other 
parameters. One of the most important applications of AVHRR data from thermal channels 3B, 4 and 5 is estimation of the global sea surface temperature (SST) [Reynolds and Smith, 1993]. The thermal channels have also been used to retrieve cloud cover information [Rossow, 1989; Trishchenko et al., 2001], land surface temperature, and thermal emissivity [Qin and Karnieli, 1999]. Forest fire detection and monitoring is also a very important application of AVHRR thermal data [Li et al., 2001]. Global climate monitoring and change detection require SST and land surface temperatures with a high accuracy. The generally accepted requirement is $\pm 0.1 \mathrm{~K}$ [Kidwell, 1998; Vazquez and Sumagaysay, 2001].

Unlike the short-wave channels (1, 2 and 3A) that are not calibrated onboard, the thermal channels (3B, 4 and 5) are calibrated in-flight using measurements of the internal calibration target (ICT) and deep-space (SP) [Cracknell, 1997; Kidwell, 1998]. The thermal state of the ICT target is monitored by four platinum resistance thermistors (PRTs). Providing that the monitoring of the ICT temperature is accurate, the measurement error is limited by the magnitude of the noise equivalent delta temperature (NE $\Delta \mathrm{T}$ ) for each thermal channel. Other factors, such as nonblackness of the ICT, i.e. departure of its emissivity from unity, and reflections from surrounding elements contribute only about $10 \%$ of the total uncertainty [Weinreb et al., 1990]. Usually, this uncertainty is estimated during pre-flight tests under laboratory conditions. Some information about the accuracy achieved during preflight ground IR calibration is available [Brown et al., 1985, 1993; Kidwell, 1998; Walton et al., 1998; Sullivan, 1999). However, the accuracy of AVHRR operational IR measurements is not well characterized. We addressed this issue by conducting an analysis of NE $\Delta \mathrm{T}$ throughout the lifetime of AVHRRs from NOAA-9 to NOAA-16 (except AVHRR/NOAA- 
10 which had an AVHRR/1 type radiometer) based on Global Area Coverage (GAC) data available from the NOAA Satellite Active Archive (SAA).

As we show below, the critical issue is the accuracy of the ICT temperature monitoring because any uncertainty in the ICT temperature directly converts into an uncertainty in the pixel brightness temperature. Our analysis shows that each PRT sensor, depending on its spatial location on the ICT, responds differently to thermal forcing. This forcing is caused by changes in solar heating and radiative cooling, which in turn depend on solar illumination conditions along an orbit and the relative position between the sun and orbital plane for a given time of the year. An analysis of the correlation between PRT temperatures reveals complex thermal processes within the ICT, which impact negatively on the accuracy of AVHRR thermal calibration.

The paper is organised as follows. Section 2 outlines the general principles of thermal calibration of AVHRR radiometers. Section 3 analyses the NE $\Delta T$ for each thermal channel. Section 4 presents an analysis of ICT temperature data. The solar contamination effect and its impact on the accuracy of thermal calibration are also addressed in this section, together with an application of a sensor response model and the derivation of the sensor's time response constant. In Section 5, long-term trends in thermal channel calibration gains are examined. Section 6 concludes the paper. 


\section{The principles of AVHRR thermal calibration}

The physical principles of AVHRR thermal calibration are described by Kidwell [1998], Cracknell [1997], and Planet [1988]. Some useful notations and formulae used throughout the paper are introduced here.

We denote the spectral response function of channel $\boldsymbol{i}$ as $\boldsymbol{r}_{\boldsymbol{i}}(\lambda)$. The normalized spectral response functions for AVHRR NOAA-14 and -16 channels 3B, 4 and 5 are presented in Figure 1. In-flight calibration of the thermal channels is based on the measurements of radiation emitted by the ICT and by deep space. The ICT operates approximately at the temperature of the radiometer's internal environment, which varies usually between $286 \mathrm{~K}$ and $300 \mathrm{~K}$. The thermal detectors are typically maintained at a temperature of $107 \mathrm{~K}$ by a radiant cooler subsystem [Cracknell, 1997].

There are 10 samples of SP and ICT counts as measured by the radiometer in every AVHRR scan line. They represent a digitized output of the radiometer when the scanning mirror views the SP and the ICT targets. The average of the 10 samples is usually used for calibration. To make calibration less sensitive to noise effects, a more robust approach is recommended [Trishchenko, 2002].

Four PRTs imbedded in the ICT are used to monitor its temperature. Three samples of digital counts from each PRT are added to the output data stream for each scan line (minor data frame). The samples from only one PRT are included in each minor data frame, creating a sequence of four data sets and followed by a null data set in the output data stream in high resolution picture transmission (HRPT) data. Therefore, digital counts are available from each PRT once for every 5 minor frames (5/6 of a second). The PRT data are used to 
uniquely determine the ICT temperature, $\boldsymbol{T}_{\mathbf{I C T} \text {. }}$ To make it consistent with PRT observations, often 50 samples (5 scanlines) instead of 10 samples (1 scanline) are used to compute the average blackbody counts. The PRT sequence in GAC data differs from that of HRPT, since only every third scan line is included in the output data stream. The radiation $\mathrm{R}_{\text {ICT }}^{\mathrm{i}}$ received by channel $\boldsymbol{i}$ detector from the ICT with unit emissivity (blackbody) is

$$
R_{I C T}^{i}=\int_{\lambda_{\min }}^{\lambda_{\max }} B\left(\lambda, T_{I C T}\right) r_{i}(\lambda) d \lambda
$$

where

$$
B(\lambda, T)=\frac{c_{1} v^{3}}{\exp \left(\frac{c_{2} v}{T}\right)-1} \text { is the Planck function, }
$$

$\boldsymbol{c}_{\mathbf{1}}=1.1910659 \times 10^{-5} \mathrm{~mW} \mathrm{~m}^{-2} \mathrm{sr}^{-1} \mathrm{~cm}^{4}$, $\boldsymbol{c}_{2}=1.438833 \mathrm{~cm}^{-} \mathrm{K}$.

The following expression is recommended for conversion of PRT counts into temperature for all 4 PRTs [Kidwell, 1998] :

$$
T_{P R T}=a 0+a 1 * P R T+a 2 * P R T^{2}+a 3 * P R T^{3}+a 4 * P R T^{4}
$$

The ICT temperature, $T_{\text {ICT }}$, is derived as an average of the 4 PRT temperatures [Kidwell, 1998]:

$$
T_{I C T}=1 / 4\left(T_{P R T 1}+T_{P R T 2}+T_{P R T 3}+T_{P R T 4}\right)
$$

To compute radiometer channel calibration gains, $\boldsymbol{G}^{(\boldsymbol{i})}$, and offsets, $\boldsymbol{I}^{(\boldsymbol{i})}$, NOAA recommends using a small non-zero radiance value for the space target radiance, $\boldsymbol{R}_{\boldsymbol{S P}}$. These 
values are instrument- and channel- specific [Kidwell, 1998]. The calibration gain and offset values are determined from the following equations:

$$
\begin{aligned}
& G^{(i)}=\frac{R_{I C T}^{(i)}-R_{S P}^{(i)}}{C_{I C T}^{(i)}-C_{S P}^{(i)}}, \\
& I^{(i)}=R_{S P}^{(i)}-G^{(i)} \cdot C_{S P}^{(i)},
\end{aligned}
$$

where

$\mathrm{C}_{\mathrm{ICT}}^{(\mathrm{i})}$ is the digital count for the internal calibration target,

$C_{S P}^{(i)}$ is the digital count for the space target.

The final step of calibration consists of a non-linear correction that converts linear radiance $\boldsymbol{R}_{\text {lin }}$ into corrected radiance $\boldsymbol{R}$ for every pixel in channel $\boldsymbol{i}$ :

$$
R^{(i)}=A^{(i)} \cdot R_{\text {lin }}^{(i)}+B^{(i)} \cdot\left(R_{l i n}^{(i)}\right)^{2}+D^{(i)}
$$

where the linear radiance is

$$
R_{\text {lin }}^{(i)}=G^{(i)} \cdot C_{P}^{(i)}+I^{(i)}
$$

Coefficients $A^{(i)}, B^{(i)}$, and $D^{(i)}$ are known constants [Kidwell, 1998; Sullivan, 1999; Goodrum et al, 2000]. $C_{P}^{(i)}$ is the observed pixel response in counts. Nonlinear corrections for NOAA-9, -11 and -12 were implemented in data processing through look-up table approach instead of applying Eq. (5) [Kidwell, 1998] 
Although similar in principle, the thermal calibration procedure is slightly different for the KLM satellite series AVHRR/3. There is an effective blackbody temperature correction and an alternate non-linear radiance correction equation as described in the NOAA KLM User’s Guide (http://www2.ncdc.noaa.gov/docs/intro.htm).

Eq. (1) assumes that all radiation observed by AVHRR thermal channel $\boldsymbol{i}$ is emitted by ICT with emissivity $\varepsilon=1$, thus neglecting its departure from a perfect blackbody. The real situation is more complicated. A small departure of $\varepsilon$ from 1 causes reflection of thermal radiation emitted by the AVHRR interior. As such, a more accurate expression would be:

$$
R_{I C T}^{i}=\int_{\lambda_{\min }}^{\lambda_{\max }}\left[\varepsilon(\lambda) B\left(\lambda, T_{I C T}\right)+(1-\varepsilon(\lambda)) L(\lambda)\right] r_{i}(\lambda) d \lambda,
$$

where $(1-\varepsilon(\lambda)) L(\lambda)$ is the total radiation reaching the ICT surface and reflected back

toward the detector. When the ICT and its environment is in approximate thermal equilibrium, i.e. $B(\lambda, T) \approx L(\lambda)$, then Eq. (7) is equal to Eq. (1).

\section{Noise equivalent error $\mathrm{NE} \Delta \mathrm{T}$}

The NE $\Delta \mathrm{T}$ error can be derived from Eqs. (1) to (5) assuming statistical independence and normal distribution of noise in the SP, ICT and PRT measurements.

The expression for the uncertainty $\delta R$ follows from Eq. (5):

$$
\delta R=\left|A+2 B R_{\text {lin }}\right| \delta R_{\text {lin }} \approx \delta R_{\text {lin }},
$$


since parameter $B$ is small, and parameter $A$ is close to 1 . In Eq. (8) we omitted index "i" denoting the channel selection.

Given the following expression for linear radiance:

$$
R_{\text {lin }}=f\left(T_{I C T}, C_{p}, C_{I C T}, C_{s p}\right)=R_{s p}+\left(R_{I C T}-R_{s p}\right) \frac{C_{p}-C_{s p}}{C_{I C T}-C_{s p}}
$$

and assuming statistical independence and normal distribution of $\delta$-variations, the total uncertainty for linear radiance can be written as:

$$
\delta R_{\text {lin }}=\sqrt{\left(f_{T_{I C T}}^{\prime}\right)^{2} \delta T_{I C T}^{2}+\left(f_{C_{I C T}}^{\prime}\right)^{2} \delta C_{I C T}^{2}+\left(f_{C_{s p}}^{\prime}\right)^{2} \delta C_{s p}^{2}+\left(f_{C_{p}}^{\prime}\right)^{2} \delta C_{p}^{2}} .
$$

The $\delta T_{\text {ICT }}$ error term in Eq. (10) is caused by noise in the PRT measurements. Assumption about statistical independence of $\delta$-variations was confirmned by data analysis that showed very low level of correlation between ICT and SP noise at the level of $10^{-3}$ to $10^{-2}$.

To determine the noise equivalent error, we analyzed the standard deviations $\delta C$ of ICT, SP and PRT counts, for every scan line within a single GAC data file. Statistics for various AVHRR radiometers are presented in Table 1. Values in Table 1 are the average standard deviations for individual GAC data files. There are two values for each parameter to show typical range of variability; they were computed for GAC data files taken at the beginning and end of instrument operations to account for possible changes in instrument measurement properties throughout its lifetime.

An example of standard deviations for AVHRR NOAA-16 on February 5, 2002 is shown in Figure 2. The left panels of Figure 2 contain SP counts and the right panels contain ICT counts. Panels from top to bottom are for channels 3B, 4 and 5. Switching to the channel 3A mode causes gaps in SP and ICT data for channel 3B in the two top panels (a-b). Channel 3A and 3B cannot operate at the same time. 
The instrument noise characteristics for SP and ICT observations are similar. These observations represent two extreme situations, corresponding to high and low radiance levels. We may assume that the noise level is also of the same magnitude for intermediate input signals. Therefore, the signal-to-noise ratio is greater for low pixel brightness temperature and smaller for higher brightness temperatures. Noise for channel 3 on AVHHR/2 varies within a range of 2 to 4 counts. The largest $\delta C$ values for channel 3 were found for NOAA9, $-12,-14$, and the smallest ones were for NOAA-11. Noise for channels 4 and 5 of AVHRR/2 are typically less than 0.5 count, except for NOAA-12 where it slightly exceeds this level. The magnitude of the noise in channel 3B of the AVHRR/3 model is much smaller and closer to values observed in channels 4 and 5. For NOAA-16, the noise in channel 5 exceeds the noise in channels 3B and 4.

Uncertainties in the PRT counts are quite small, causing a negligible error in pixel brightness temperature (less than $\pm(0.01$ to 0.02$) \mathrm{K})$. We neglect this error by omitting the first term in Eq. (10). Note that the actual accuracy of ICT temperature measurements is not identical to the PRT noise error; this will be discussed further.

Let us assume that the noise error for each type of calibration sample data and pixel count is the same, and denoted as $\delta C$. Eq. (10) then becomes

$$
\delta R_{\text {lin }}=\delta C \sqrt{\left(f_{C_{\text {ICT }}}^{\prime}\right)^{2}+\left(f_{C_{s p}}^{\prime}\right)^{2}+\left(f_{C_{p}}^{\prime}\right)^{2}} .
$$

The derivatives are given by

$$
f_{C_{I C T}}^{\prime}=-G \frac{C_{p}-C_{S P}}{C_{I C T}-C_{S P}} ; \quad f_{C_{S P}}^{\prime}=G \frac{C_{p}-C_{I C T}}{C_{I C T}-C_{S P}} ; \text { and } \quad f_{C_{p}}^{\prime}=G \text {, }
$$

where the gain $G$ is determined by Eq. (4a). By introducing the variable 
$\varepsilon=\frac{C_{p}-C_{S P}}{C_{I C T}-C_{S P}}$,

we obtain the following expression for $\delta R_{\text {lin }}$ :

$\delta R_{\text {lin }}=\sqrt{2} G(\delta C) \sqrt{1-\varepsilon(1-\varepsilon)}$.

The NE $\Delta$ T error computed according to Eq. (14) is presented in Figure 3. The panels from top to bottom are for channel 3B (a), channel 4 (b) and channel 5 (c). The NE $\Delta \mathrm{T}$ error is shown as a function of pixel brightness temperature. In the calculations, we employed average numbers for the standard deviation $\delta C$ shown in Table 1. Eq. (14) also contains the calibration gain $\boldsymbol{G}$. We used average values of gain over the instrument lifetime, presented in Table 2. The NE $\Delta \mathrm{T}$ for channels 4 and 5 is within the NOAA established threshold of 0.12 $\mathrm{K}$ at $300 \mathrm{~K}$ for all radiometers considered [Kidwell, 1998], although NE $\Delta \mathrm{T}$ for channel 5 of NOAA-16 is slightly larger $(\sim 0.13 \mathrm{~K})$. The NE$\Delta \mathrm{T}$ for channel $3 \mathrm{~B}$ is noticeably larger than the threshold $(0.12 \mathrm{~K})$ except for AVHRR NOAA-15 and -16 . The NE $\Delta \mathrm{T}$ values for channel 3B may be as high as $0.3 \mathrm{~K}$ (NOAA-9 and -12). When the brightness temperature of a pixel decreases, the NE $\Delta \mathrm{T}$ error sharply increases; it may be as high as $0.2 \mathrm{~K}$ for channels 4 and 5 and $1-3 \mathrm{~K}$ at $250 \mathrm{~K}$ for channel 3B. The NE$\Delta \mathrm{T}$ errors for channels 4 and 5 of AVHRR NOAA-15 and -16 are larger than the NE $\Delta \mathrm{T}$ errors of channel 4 and 5 for all other considered radiometers. The corresponding NEAT error for channel 3B of AVHRR NOAA15 and -16 is the smallest among channel 3B NE $\Delta \mathrm{T}$ errors. 


\section{Accuracy of the ICT temperature monitoring}

Pre-flight testing and calibration is performed when the instrument is in a state of thermal equilibrium [Walton et al., 1998]. In the case of in-flight operation, the radiometer temperature experiences periodic oscillations caused by the satellite moving in and out of sunlight during an orbit. It is generally believed that the AVHRR calibration system can handle these circumstances correctly, although we are not aware of any ground tests to support this. Another type of variation is caused by solar blackbody contamination, described by Steyn-Ross et al. [1992], Kidwell, [1998], Cao et al. [2001] and analyzed by Trishchenko and $\mathrm{Li}$ [2001]. It results from sunlight impinging on the AVHRR interior during calibration scans on certain parts of the orbit. This short-term radiative impact may last only a few minutes but can induce a significant perturbation in the calibration data, comparable to or larger than the total range of variation during the entire orbital cycle. Therefore, a major question that has not been addressed so far is: what is the accuracy of in-flight calibration under a variable thermal environment?

In principle, the calibration system is supposed to handle all possible changes in the state of an instrument. In practice, however, it is not always possible to do so. The limiting factor seems to be the accuracy of the ICT temperature monitored by the PRTs. Figure 4 shows the variations in PRT temperatures within a single orbit for AVHHR radiometers. Each of 6 panels shows four PRT temperatures for each radiometer. An important point here is that the amplitude of ICT temperature variations within a single orbit may easily reach several degrees. The spread among temperature curves corresponding to individual PRT sensors is also quite significant. Such behavior convincingly demonstrates the existence of temperature 
variations and inhomogeneity of the temperature field within the ICT. The data for an individual orbit may differ from the results shown in Figure 4 in either way due to seasonal cycle and systematic orbit precession throughout of satellite lifetime, however we believe that Figure 4 gives a quite fair picture of observed perturbations.

Figure 5 summarizes the variations in instrument thermal state throughout the lifetime of each instrument with four panels. The top panel (a) shows changes in average ICT temperature over the entire instrument lifetime. Long-term trends superimposed with seasonal type variations are evident. Panel (b) displays the amplitude ( $\left.\Delta \mathrm{T}=\mathrm{T}_{\max }-\mathrm{T}_{\min }\right)$ of ICT temperature variation within a single orbit. The ICT temperature in panel (b) is computed according to Eq. (3) by averaging PRT temperatures. Panels (c) and (d) show respectively the minimum and maximum spread among four PRT temperatures within a single orbit. Variations in the ICT thermal state within a single orbit may reach 7 K (AVHRR NOAA-12) as shown in panel (b). For the AVHRR/2 radiometers in general, this parameter fluctuates between $2.5 \mathrm{~K}$ and $7 \mathrm{~K}$. For AVHRR/3 (NOAA-15, -16), the variation in the ICT temperature is smaller ( 1 to $3 \mathrm{~K}$ ). This attests to the important fact that calibration of the AVHRR thermal channels takes place under non-stationary thermal conditions, with two consequences. First, the temperature of the massive calibration target (diameter $\sim 20 \mathrm{~cm}$, [Cracknell, 1997]) cannot be monitored precisely in the presence of time-dependent thermal gradients, unless the PRTs have a very short response time to reach thermal equilibrium. Second, non-linear relationships exist between PRT temperatures, which lead to errors in determining ICT temperatures through the linear average given by Eq (3).

Figure $5 c$ indicates that AVHRR radiometers aboard NOAA-9, -15 and -16 have a small spread among PRT temperatures (typically less than $0.2 \mathrm{~K}$ ), but the spread for others is much 
larger, up to $1.5 \mathrm{~K}$ for AVHRR NOAA-12. The difference persists over time, an indicator of possible bias in PRT calibration or persistent temperature gradients across the ICT surface. In either case, this impairs the thermal calibration accuracy. One may consider the global minimum of the spread as an indicator of the accuracy of PRT temperature monitoring. Table 3 contains the overall minimum values and the average and median single orbit minimum spreads computed over the instrument lifetime. The analysis suggests a systematic bias of $\sim 0.3 \mathrm{~K}$ in the ICT temperature determination for AVHRR aboard NOAA-11 and -14 .

If the temporal variations in PRT temperature are significant in a state of thermal nonequilibrium, the behavior of a detector must be described by a PRT/ICT system response time model to determine the actual temperature of the ICT. We propose to apply the following model [Brock, 2001]:

$$
T^{\prime}(t)=\frac{T_{0}(t)-T(t)}{\tau}
$$

where $T^{\prime}(t)$ is time derivative of the measured ICT temperature, $T(t)$;

$T_{0}(t)$ is true temperature of ICT surface;

$\tau$ is PRT/ICT system response time constant.

t denotes time.

The physical meaning of Eq. (15) is that the difference between sensor-reported and actual ICT temperatures is proportional to the rate of temperature change and the PRT/ICT system response time constant. This parameter characterizes the sensor's ability to respond promptly to changes in ICT temperature. Sensors with a large response time constant react slowly to temperature changes, thus introducing a delay and distortion in the temporal temperature change. To follow closely the real ICT temperature, the sensor response time 
must be small. Fully accurate measurements are possible only when $\tau$ is zero or at least much smaller than the perturbation period.

Eq. (15) provides a simple solution for the true temperature:

$$
T_{0}(t)=T(t)+\tau T^{\prime}(t)
$$

Eq. (16) includes a component proportional to the PRT response time constant and rate of temperature change $T^{\prime}(t)$ to characterize the sensor thermal inertia. This model is appropriate for thermal channels 4 and 5, which are not sensitive to solar radiation. The model can also be applied to channel 3B only when no solar contamination of the ICT measurements occurs. In the case of solar contamination of channel 3B, Eqs. (15-16) are not applicable because they do not account for any external source of radiation, such as stray light or reflected solar radiation. A method based on Fourier transform filtering technique [Trishchenko and Li, 2001; Trishchenko, 2002] may be applied in this later case. There are indications that solar contamination may also partially affect the Earth view observations [Kilpatrick et al., 2001].

To estimate the value of the PRT response time constant, we analyzed the time derivative of the ICT temperature and channel 4 and 5 gains. The results indicate that during solar radiative contamination, channel gain and the derivative of the ICT temperature $T^{\prime}(t)$ experience synchronous perturbation. Once we apply the PRT/ICT system response time model and Eq (16), the spikes in the instrument gain can be eliminated by choosing an appropriate time constant. This is illustrated in Figure 6 for channel 4 gains. The results for channel 5 (not shown) were similar. The time constants $\tau$ were derived by computing series of instrument gain for various $\tau$ (from 5 sec to 2 minutes) and choosing $\tau$ that removed unphysical variation of calibration gain during the period of solar contamination. Figure 6 
shows panels for each AVHRR under consideration, with three curves per panel: 1) calibration gain taken from GAC data file which was computed according to the standard definition given by Eq. (4); 2) time derivative of the ICT temperature determined according to Eq. (3); and 3) calibration gain computed with the corrected ICT temperature from Eq. (16). A high degree of correlation between the original gain and the time derivative of the ICT temperature is observed. Assuming that spikes in the original gain profile are caused by thermal inertia effects described by Eqs. (15) and (16), the PRT time response can be estimated.

The values of the PRT response time constant that remove spikes in the gain profiles for a GAC orbit are $\sim 30( \pm 3)$ sec for the AVHRR/2 radiometers, $\sim 90( \pm 9)$ sec for AVHRR/3 NOAA-15 and $\sim 75( \pm 7)$ sec AVHRR/3 NOAA-16. Values of $T^{\prime}(t)$ were derived after applying Fourier transform filtering (FTF) to the ICT temperature time series to remove random noise and to apply curve smoothing necessary for numerical differentiation. The cutoff time period in the FTF procedure was set to one minute [Trishchenko, 2002]. The results for all AVHRR radiometers under consideration (Figure 6) confirm that thermal inertia of PRT measurements is the major source of calibration gain perturbation. There are still some irregular variations in calibration gain after applying the correction. These may represent uncertainties arising from numerical differentiation of a discrete function and from non-linear effects during the ICT heating/cooling, neither of which are accounted for in Eq. (3).

Analysis of ICT temperature values indicates that the ICT temperature is monitored with an uncertainty greater than the documented NE $\Delta \mathrm{T}$ error. Two major sources of this uncertainty are the variable thermal state of the ICT, and possible inconsistencies in PRT calibration. The investigation of specific sources and quantities of errors is beyond of the 
scope of this study, since it cannot be based solely on available in-flight calibration measurements. The uncertainty due to the variable thermal state of the ICT includes two kinds of errors due to: 1) the thermal inertia of the PRT system and 2) a non-linear relationship between mean ICT temperature used in the calibration and the individual temperatures reported by the PRTs. The thermal inertia of the PRT/ICT system may introduce errors of $\pm 0.5 \mathrm{~K}$ or more (Figure 6). A substantial spread between individual PRT temperatures (Figure 5) supports the hypothesis that a significant thermal gradient may exist across the ICT surface. The ICT used currently in the calibration of the AVHRR radiometers has a geometrical size $(\sim 20 \mathrm{~cm})$, which is close to the width of the entire AVHRR system [Cracknell, 1997]. The thermal state of such a massive and extended calibration target is difficult to control under non-stationary thermal conditions. This is especially true for AVHRR/2 system, where inconsistencies between PRT sensors are large. Inconsistencies between various PRTs for the AVHRR/3 system are much smaller.

\section{Long-term trends in thermal calibration coefficients}

Temporal variations occur in the instrument calibration gain coefficients associated with spacecraft orbital motion (Figures 4 and 6). They are linked to fluctuations in the AVHRR thermal state caused by cycles of solar heating followed by thermal cooling. Temporal variations of longer periods also exist. They represent a seasonal cycle related to the change

in the sun's position relative to the spacecraft orbital plane, as well as a systematic radiometric drift (degradation) of the calibration system. The mean ICT temperatures and 
calibration gains fluctuate during the lifetime of an instrument (Figure 7). The average temperature per orbit is shown in Figure 7(a) and the calibration gains for channels 3B, 4 and 5 are shown in Figures 7(b) to 7(d), respectively. Data points in Figure 7 represent averages of a single orbit taken approximately once a month except for AVHRR/NOAA-16, where orbits were sampled more frequently because of the shorter time interval of available data. Long-term changes due to sensor degradation are evident in these panels.

To separate the contribution due to seasonal temperature changes from that due to sensor degradation, we applied a two-parameter linear regression model:

$$
G(T, t)=G_{0}+G_{T} \cdot\left(T-T_{\text {mean }}\right)+G_{t} \cdot\left(t-t_{0}\right),
$$

where $T$ denotes temperature and $t$ time. The coefficient $G_{T}$ determines the influence of temperature on the calibration gain, and $G_{t}$ represents the level of sensor degradation with time. The estimates of the magnitude for coefficients $G_{T}$ and $G_{t}$, and the $r^{2}$ statistics for the two-parameter linear regression model (Eq. 17) are given in Table 4. Except for AVHRR/NOAA-9 and channel 4 of NOAA-12, the discussed regression model explains very large part of the variance, from $80 \%$ to $99 \%$. Therefore, in general the model is indeed justified. Smaller $r^{2}$ values for NOAA-9 and ch.4 of NOAA-12 may be explained by the strongest observed solar blackbody contamination effect for these radiometers.

Results presented in Table 4 show that the ICT temperature exerts a positive influence (reducing the absolute magnitude of the negative gain) on channel 3B calibration gain. With a few exceptions, the ICT temperature exerts a negative influence (increasing the magnitude of the negative gain) on channel 4 and 5 gains. Exceptions were observed for channel 5 of AVHRR/NOAA-11 and AVHRR/NOAA-14, where $G_{T}$ has small positive values of 
$+0.01 \% \mathrm{~K}^{-1}$ and $+0.11 \% \mathrm{~K}^{-1}$, respectively. Sensor temporal changes characterized by coefficient $G_{t}$ are negative for channel 3B in all AVHRR radiometers studied. They are also mostly negative for channels 4 and 5 with the exception of channel 4 of AVHRR/NOAA-9 and -16, and channel 5 of AVHRR/NOAA-15.

A negative temporal trend indicates a degradation of the sensor's sensitivity with time. Except for channel 3B of AVHRR/NOAA-16, the rate of degradation is between $-0.32 \% \mathrm{yr}^{-1}$ and $-0.85 \% \mathrm{yr}^{-1}$. The value for AVHRR/NOAA-16 $\left(-1.4 \% \mathrm{yr}^{-1}\right)$ must be interpreted with caution since it is derived from a small sample covering less than one year. While degradation of sensor sensitivity with time is quite an understandable phenomena, the temperature dependence of calibration gain reported in this paper is quite an interesting new fact, that has no trivial explanation.

The calibration gain determines the scale of transformation between instrument counts and physical quantities. As such, the variation in gain affects the measurement limits. The zero radiance level is linked to the SP signal; therefore the lower observational level is always fixed. Since the observable radiance dynamic range $\Delta R$ is proportional to $G \Delta C$, where $\Delta C=1023$ is the interval of measured counts for 10-bit data, variability in gain results in the migration of the maximum observable radiance and brightness temperature.

The dynamic range of brightness temperatures that can be detected in channels 4 and 5 exceeds that observed for typical targets at the top-of-the-atmosphere (TOA) level, and consequently these channels do not reach the saturation limit. However, that is not the case for channel 3B in which both the solar reflected and thermal emitted radiation are significant. The equivalent brightness temperature of the solar constant in channel 3B is about $350 \mathrm{~K}$ to $360 \mathrm{~K}$. As such, the sum of solar reflected and thermal emitted components may saturate the 
channel 3B detector. In particular, the saturation limit of channel 3B is important for remote sensing of forest fires and cloud microphysical properties. Figure 8 shows average values of maximum observable temperatures for a single orbit in channel 3B. Upper and lower limits within a single orbit are also shown in Figure 8 as dotted curves, and the range between maximum and minimum values is marked as vertical arrows. Variations in the maximum observable temperature for AVHRR/NOAA-9, -11,-12 within a single orbit may reach approximately $\pm 1.5 \mathrm{~K}$. This is mostly caused by solar contamination of the ICT observations in GAC data [Trishchenko and Li, 2001; Cao et al., 2001; Trishchenko, 2002]. Variations within a single orbit are significantly smaller for other radiometers (less than $\pm 0.5 \mathrm{~K}$ ).

The maximum observable temperature in channel 3B for AVHRR/3 (NOAA-15, -16) is significantly higher than for AVHRR/2 (NOAA-9, -11, -12, -14). It is around $336 \mathrm{~K}$ to $337 \mathrm{~K}$ for $\mathrm{AVHHR} / 3$ versus $321.5 \mathrm{~K}$ to $322.5 \mathrm{~K}$ for $\mathrm{AVHRR} / 2$. As such, the $\mathrm{AVHRR} / 3$ reaches saturation at higher brightness temperatures and is more suited for remote sensing studies that require wider dynamical range.

\section{Summary}

Observations made in AVHRR thermal channels are important for weather forecasting and climate monitoring. Despite their significance and extensive applications, the accuracy of the in-flight calibration had not been rigorously investigated. This study attempted to gain insight into some particular calibration issues, including in-flight noise equivalent error 
(NE $\Delta \mathrm{T}$ ), the consistency of PRT-based monitoring of the Internal Calibration Target (ICT) temperature, and long-term variations in calibration gains and dynamic range of the AVHRR.

The study focused on the analysis of AVHRR/2 (NOAA-9, -11, -12, -14) and AVHRR/3 (NOAA-15,-16) radiometers. We utilized GAC data, approximately one orbit per month throughout the lifetime of the instruments, from the NOAA Satellite Active Archive (SAA).

The overall finding of the error budget study is that AVHRR calibration measurement uncertainties are somewhat higher than assumed previously and exceed the level $\pm 0.1 \mathrm{~K}$ required for climate change monitoring, even for new the AVHRR/3 (KLM series) radiometers.

Analysis of the NE $\Delta \mathrm{T}$ error revealed that the threshold of $0.12 \mathrm{~K}$ at $300 \mathrm{~K}$ as stated by the instrument manufacturer was met for channels 4 and 5 most of the time; while for channel 3B of AVHRR/2 it was generally higher with a value of $0.15 \mathrm{~K}-0.3 \mathrm{~K}$. The NEAT error increases significantly at lower temperatures.

Analysis of the PRT measurements uncovered complex thermal fluctuations within the ICT. PRT observations show much better consistency for the AVHRR/3 radiometer than for AVHRR/2. This finding calls for a careful reanalysis of calibration data from AVHRR/2 radiometers to produce historical thermal data sets for climate change studies.

A major source of error in the calibration data is solar contamination of the ICT. The current calibration approach, as implemented in the AVHRR data processing, introduces large uncertainties (up to $\pm 0.5 \mathrm{~K}$ and more) due to thermal inertia effects from rapid changes in the ICT temperature. We proposed a physical model that accounts for the thermal inertia effect. An estimated value for the AVHRR response time constant $\tau$ is around 0.5 minute for 
AVHRR/2 and around 1.25-1.5 minute for AVHRR/3. We estimate the uncertainty of the derived response time constant values to be between 5 and 9 sec or around $10 \%$. The model is recommended for operational use to improve thermal calibration accuracy and to better correct the influence of solar contamination. This technique also requires the implementation of a special smoothing procedure to obtain accurate results for the time derivative of ICT temperature $T^{\prime}(t)$.

Long-term trends in the calibration gains of IR channels 3 to 5 were evaluated. In general, a small degradation of the sensor radiometric sensitivity was detected in almost all cases, though the rate of degradation is usually quite low (typically below $0.85 \%$ year $^{-1}$ ), except for channel 3B of AVHRR/NOAA-16. Data for the latter case must be interpreted with caution due to a short observation interval. Variation in calibration gain leads to fluctuations in maximum observable target brightness temperature (upper bound of the dynamic range of temperatures that can be measured by the sensor). This is especially important for channel 3B, which may saturate during daytime and for forest fire hot spot pixels. The maximum observable temperature in channel 3B of AVHRR/2 varies between 321.5 K and 322.5 K. Newly launched radiometers show an increased maximum temperature limit of $336 \mathrm{~K}-337 \mathrm{~K}$.

The incorporation of the derived results and proposed improvements into AVHRR thermal calibration should result in better accuracy of derived datasets, improved consistency between various AVHRR missions, and lead to more reliable estimates of long-term changes in the Earth’s climate system.

Acknowledgments. The authors thank William Park (CCRS) for technical assistance with HRPT data handling. The use of AVHRR GAC data acquired through the NOAA SAA is 
gratefully acknowledged. This research was partially supported by the Biological and Environmental Research Program (BER), U.S. Department of Energy, Grant No. DE-FG0202ER63351. 


\section{References}

Brock, F. V., Meteorological measurement systems. New York: Oxford University Press, 290pp. 2001.

Brown, O.B., J.W. Brown, and R. H. Evans, Calibration of Advanced Very High Resolution Radiometer infrared observations. J. Geophys. Res., 90, 11667-11677, 1985.

Brown, J.W., O. B. Brown, and R. H. Evans, Calibration of advanced very high resolution radiometer infrared channels: A new approach to nonlinear correction. J. Geophys. Res., 98, 18257-18268, 1993.

Cao, C., M. Weinreb, J. Sullivan, Solar contamination effects on the infrared channels of the advanced very high resolution radiometer (AVHRR). J. Geophys. Res., D106: 33,46333,469, 2001.

Cihlar, J., Land Cover Mapping of Large Areas from Satellites: Status and Research Priorities; Int. J. Remote Sensing, 21 , 1093 - 1114, 2000.

Cihlar, J., R. Latifovic, J. Chen, A. P. Trishchenko, Y. Du, G. Fedosejevs, and B. Guindon, Systematic corrections of AVHRR image composites for temporal studies, Remote Sens. Environ. (in press), 2002.

Cracknell, A. P. The advanced very high resolution radiometer (AVHRR). Taylor \& Francis, London, 534 pp, 1997.

Goodrum, G., K. B. Kidwell, W. Winston (Eds), NOAA KLM User’s Guide. Revised. US Department of Commerce, NESDIS, NOAA, National Climatic Data Center, Satellite Data Services Division, Washington, DC, USA, 2000. 
Ignatov, A., and L. Stowe, Aerosol retrievals from individual AVHRR channels. Part I: Retrieval algorithm and transition from Dave to 6S radiative transfer model. J. Atmos. Sci., 59, 313-334, 2002a.

Ignatov, A., and L. Stowe, Aerosol retrievals from individual AVHRR channels. Part II: Quality control, probability distribution functions, information content, and consistency checks of retrievals. J. Atmos. Sci., 59, 335-362, $2002 \mathrm{~b}$.

Kidwell, K. B. (editor), NOAA Polar Orbiter Data User's Guide. US Department of Commerce, NESDIS, NOAA, National Climatic Data Center, Satellite Data Services Division, Washington, DC, USA, 1998.

Kidwell, K. B. Global Vegetation Index User's Guide, U.S. Dept. of Commerce, NOAA/NESDIS, Washington, D.C. 1990

Kilpatrick, K. A., G. P. Podestá, and R. Evans, Overview of the NOAA/NASA advanced very high resolution radiometer Pathfinder algorithm for sea surface temperature and associated matchup database. J. Geophys. Res., 106: 9179-9197. 2001.

Li, Z., Y. J. Kaufman, C. Itchoku, R. Fraser, A. P. Trishchenko, L. Giglio, J. Jin, X. Yu, A review of AVHRR-based active fire detection algorithms: Principles, limitations, and recommendations, in Global and Regional Vegetation Fire Monitoring from Space, Planning and Coordinated International Effort (Eds. F. Ahern, J.G. Goldammer, C. Justice), pp 199-225, SPB Academic Publishing, The Hague, The Netherlands. 2001.

Li, Z., L. Moreau, and J. Cihlar, Estimation of photosynthetically active radiation absorbed at the surface. J. Geophys. Res., 102: 29 717-29 727. 1997.

Planet, W. G. (Editor), Data Extraction and Calibration of TIROS- N/NOAA Radiometers, NOAA Technical Memorandum NESS 107 Revision 1, National Oceanic and 
Atmospheric Administration, Washington, D. C. (Amendments to NOAA Technical Memorandum 107; Appendix-B for NOAA-J/14). 130 pp. 1988.

Qin, Z., and A. Karnieli, Progress in the remote sensing of land surface temperature and ground emissivity using NOAA-AVHRR data. Int. J. Remote Sensing, 20: 23672393, 1999.

Rao, P.K., S. J. Holmes, R. K. Anderson, J. S. Winston, and P. E. Lehr, editors. Weather Satellites: Systems, Data, and Environmental Applications, American Meteorological Society, Boston, Mass. USA. 1990.

Reynolds, R.W., T. M. Smith, Improved global sea surface temperature analysis using optimum interpolation. J. Clim.. 7, 929-948. 1993.

Rossow, W.B., Measuring cloud properties from space: A review. J. Clim., 2: 201-213. 1989

Steyn-Ross. D. A., M. L. Steyn-Ross, and S. Clift, Radiance calibration of Advanced Very High Resolution Radiometer infrared channels. J. Geophys. Res., 97, 5551-5568, 1992.

Stowe, L. L., A. M. Ignatov, and R. R. Singh, Development, validation, and potential enhancements to the second-generation operational aerosol product at the National Environment Satellite, Data, and Information Service of the National Oceanic and Atmospheric Administration. J. Geophys. Res., 102: 16,923-16,934, 1997.

Sullivan, J., New radiance-based method for AVHRR thermal channel nonlinearity corrections. Int. J. Remote Sensing, 20, 3493-35-1, 1999.

Trishchenko, A. P., Removing unwanted fluctuations in the AVHRR thermal calibration data using robust techniques. J. Atmos. Oceanic Tech.. In press, 2002 
Trishchenko, A.P., J. Cihlar, and Z. Li. Effects of spectral response function on the surface reflectance and NDVI measured with moderate resolution sensors, Rem. Sens. Environ., 81, 1-18, 2002.

Trishchenko A. P., Z. Li, A method for the correction of AVHRR onboard IR calibration in the event of short-term radiative contamination. Int. J. Remote Sensing, 22, 36193624, 2001.

Trishchenko, A. P., Z. Li, F.-L. Chang, H. Barker, Cloud optical depths and TOA fluxes: Comparison between satellite and surface retrievals from multiple platforms, Geophys. Res. Let., 979-982, 2001.

Vazquez, J., and R. Sumagaysay, A comparison between sea surface temperatures as derived from the European remote sensing Along-Track Scanning Radiometer and the NOAA/NASA AVHRR ocean Pathfinder dataset. Bull. Amer. Meteorol. Soc., 82, 925-944, 2001.

Walton, C.C., J. T. Sullivan, C. R. N. Rao, and M. P. Weinreb, Corrections for detector nonlinearities and calibration inconsistencies of the infrared channels of the advanced very high resolution radiometer. J. Geophys. Res., 103, 3323-3337, 1998. 


\section{Figure captions}

Figure 1. Spectral response functions for the AVHRR thermal channels 3B (a), 4 (b) and 5 (c) onboard NOAA-14 (solid line) and NOAA-16 (dotted line).

Figure 2. Standard deviations of SP counts for channels $3 B$ (a), 4 (b) and 5 (c) and ICT counts for channels 3B (d), 4 (e) and 5 (f) for AVHRR NOAA-16. Data are for February 5, 2002. Gaps for channel 3B SP and ICT data correspond to the periods of channel 3A activation, when channel 3B is turned off.

Figure 3. The NEAT error versus pixel brightness temperature for various AVHRR radiometers computed according to Eq (14) for channel 3B (a), channel 4 (b), -and channel 5 (c).

Figure 4. Variation in PRT temperatures for a single GAC data file. Solar contamination is observed as a sudden jump in temperature. Temperatures for individual PRT sensors are shown for NOAA-9 (a), NOAA-11 (b), NOAA-12 (c), NOAA-14 (d), NOAA-15 (e) and NOAA-16 (f). Year and day of year (DOY) are also shown.

Figure 5. Various parameters describing the ICT thermal state:

(a) Single orbit average ICT temperature during the AVHRR lifetime;

(b) Variation of ICT temperature within a single orbit $\left(\Delta \mathrm{T}=\mathrm{T}_{\max }-\mathrm{T}_{\min }\right)$;

(c) Minimum spread among the PRT temperatures within a single orbit;

(d) Maximum spread among the PRT temperatures within a single orbit.

Figure 6. Variation in channel 4 gain $\mathrm{G}_{4}$ and ICT temperature derivative. Uncorrected (dashed) and corrected, according to Eq. (16), (solid line) values of gain $\mathrm{G}_{4}$ are 
plotted for NOAA-9 (a), NOAA-12 (b), NOAA-14 (c), NOAA-15 (d) and NOAA16 (e). Derivative $T^{\prime}(t)$ is plotted as dash-dot line.

Figure 7. Long-term changes in (a) single-orbit mean ICT temperature and single-orbit mean calibration gain for NOAA-9 to-16 AVHRR channels 3B (b), 4 (c) and 5 (d).

Figure 8. Maximum observable temperature in channel 3B for AVHRR/2(NOAA-9,-11,-12,14) and AVHRR/3 (NOAA-15, -16) radiometers. Upper and lower dotted curves indicate a range of variation within single orbit. 


\section{Table captions}

Table 1. Statistics of noise in the AVHRR thermal calibration measurements

Table 2. Estimated average values of the AVHRR channel gains used in computation of NE $\Delta T$.

Table 3. Statistics of minimum spread between PRT temperatures within a single orbit over the instrument lifetime.

Table 4. Coefficients of linear regression for the AVHRR calibration gains 
Table 1.

Statistics of noise in the AVHRR thermal calibration measurements.

\begin{tabular}{|l|c|c|c|c|c|c|c|}
\hline \multirow{2}{*}{ Platform } & \multicolumn{2}{|c|}{ Channel 3B } & \multicolumn{2}{c|}{ Channel 4 } & \multicolumn{2}{c|}{ Channel 5 } & \multirow{2}{*}{ PRT } \\
\cline { 2 - 7 } & SP & ICT & SP & ICT & SP & ICT & \\
\hline NOAA-9 & $2.2-4.2$ & $2.0-3.9$ & $0.1-0.3$ & $0.2-0.3$ & $0.4-0.6$ & $0.5-0.6$ & $0.1-0.2$ \\
\hline NOAA-11 & $1.6-2.1$ & $1.5-2.3$ & $0.1-0.2$ & $0.1-0.2$ & $0.4-0.5$ & $0.4-0.5$ & $0.1-0.2$ \\
\hline NOAA-12 & $2.8-3.9$ & $2.3-3.9$ & $0.5-0.6$ & $0.6-0.7$ & $0.5-0.6$ & $0.4-0.5$ & $0.2-0.3$ \\
\hline NOAA-14 & $2.5-3.2$ & $2.3-3.1$ & $0.2-0.5$ & $0.2-0.3$ & $0.4-0.5$ & $0.4-0.5$ & $0.3-0.4$ \\
\hline NOAA-15 & $0.7-0.9$ & $0.6-0.7$ & $0.03-0.1$ & $0.1-0.2$ & $0.3-0.4$ & $0.3-0.4$ & $0.1-0.2$ \\
\hline NOAA-16 & $0.4-0.5$ & $0.4-0.5$ & $0.5-0.6$ & $0.4-0.5$ & $0.7-0.8$ & $0.6-0.7$ & $<0.1$ \\
& & & & & & & \\
\hline
\end{tabular}

Results are given in counts. 
Table 2.

Estimated average values of the AVHRR channel gains used in computation of NE $\Delta T$.

\begin{tabular}{|l|c|c|c|}
\hline Platform & $\begin{array}{c}\text { Channel 3B } \\
{\left[\mathrm{mWm}^{-2} \mathrm{str}^{-1} \mathrm{count}^{-1} \mathrm{~cm}\right]}\end{array}$ & $\begin{array}{c}\text { Channel 4 } \\
{\left[\mathrm{mWm}^{-2} \mathrm{str}^{-1} \mathrm{count}^{-1} \mathrm{~cm}\right]}\end{array}$ & $\begin{array}{c}\text { Channel 5 } \\
{\left[\mathrm{mWm}^{-2} \mathrm{str}^{-1} \mathrm{count}^{-1} \mathrm{~cm}\right]}\end{array}$ \\
\hline NOAA-9 & -0.00145 & -0.165 & -0.195 \\
\hline NOAA-11 & -0.00150 & -0.175 & -0.180 \\
\hline NOAA-12 & -0.00165 & -0.160 & -0.180 \\
\hline NOAA-14 & -0.00162 & -0.165 & -0.180 \\
\hline NOAA-15 & -0.00240 & -0.200 & -0.215 \\
\hline NOAA-16 & -0.00245 & -0.185 & -0.195 \\
\hline
\end{tabular}


Table 3.

Statistics of minimum spread between PRT temperatures within a single orbit over the instrument lifetime.

\begin{tabular}{|l|c|c|c|}
\hline \multicolumn{1}{|c|}{ Platform } & $\begin{array}{c}\text { Overall minimum } \\
{[\mathrm{K}]}\end{array}$ & $\begin{array}{c}\text { Average single } \\
\text { orbit minimum } \\
\text { spread [K] }\end{array}$ & $\begin{array}{c}\text { Median single } \\
\text { orbit minimum } \\
\text { spread [K] }\end{array}$ \\
\hline NOAA-9 & 0.01 & 0.09 & 0.08 \\
\hline NOAA-11 & 0.31 & 0.45 & 0.36 \\
\hline NOAA-12 & 0.10 & 0.67 & 0.49 \\
\hline NOAA-14 & 0.31 & 0.59 & 0.47 \\
\hline NOAA-15 & 0.07 & 0.14 & 0.12 \\
\hline NOAA-16 & 0.04 & 0.07 & 0.07 \\
\hline
\end{tabular}


Table 4.

Coefficients of linear regression for the AVHRR calibration gains

\begin{tabular}{|c|c|c|c|}
\hline Spacecraft & $G_{T}[\% / \mathrm{K}]$ & $G_{t}[\% /$ Year $]$ & $r^{2}$ \\
\hline & \multicolumn{3}{|c|}{ Channel 3B } \\
\hline NOAA-09 & 0.28 & -0.81 & 0.49 \\
\hline NOAA-11 & 0.10 & -0.32 & 0.48 \\
\hline NOAA-12 & 0.18 & -0.34 & 0.81 \\
\hline NOAA-14 & 0.73 & -0.81 & 0.92 \\
\hline NOAA-15 & 0.02 & -0.85 & 0.92 \\
\hline \multirow[t]{2}{*}{ NOAA-16 } & 0.07 & -1.49 & 0.99 \\
\hline & \multicolumn{3}{|c|}{ Channel 4} \\
\hline NOAA-09 & -0.35 & 0.38 & 0.23 \\
\hline NOAA-11 & -0.57 & -0.44 & 0.99 \\
\hline NOAA-12 & -0.30 & -0.08 & 0.63 \\
\hline NOAA-14 & -0.01 & -0.33 & 0.93 \\
\hline NOAA-15 & -0.45 & -0.17 & 0.98 \\
\hline \multirow[t]{2}{*}{ NOAA-16 } & -0.34 & 0.17 & 0.81 \\
\hline & \multicolumn{3}{|c|}{ Channel 5} \\
\hline NOAA-09 & -0.04 & -0.50 & 0.42 \\
\hline NOAA-11 & 0.01 & -0.76 & 0.92 \\
\hline NOAA-12 & -0.12 & -0.26 & 0.77 \\
\hline NOAA-14 & 0.11 & -0.46 & 0.98 \\
\hline NOAA-15 & -0.31 & 0.25 & 0.84 \\
\hline NOAA-16 & -0.07 & -0.47 & 0.74 \\
\hline
\end{tabular}


Figure 1.

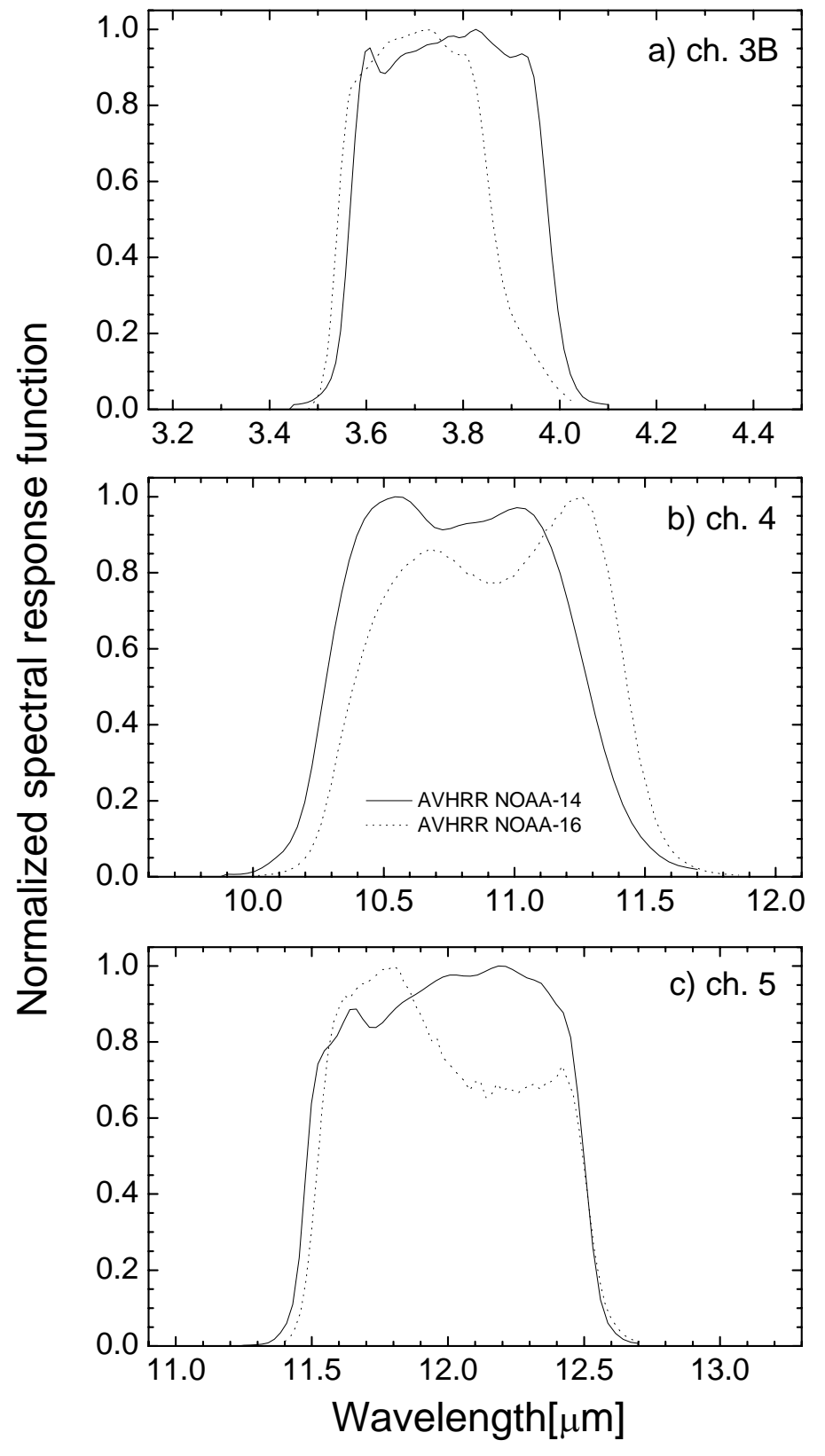

Figure 1. 
Figure 2.
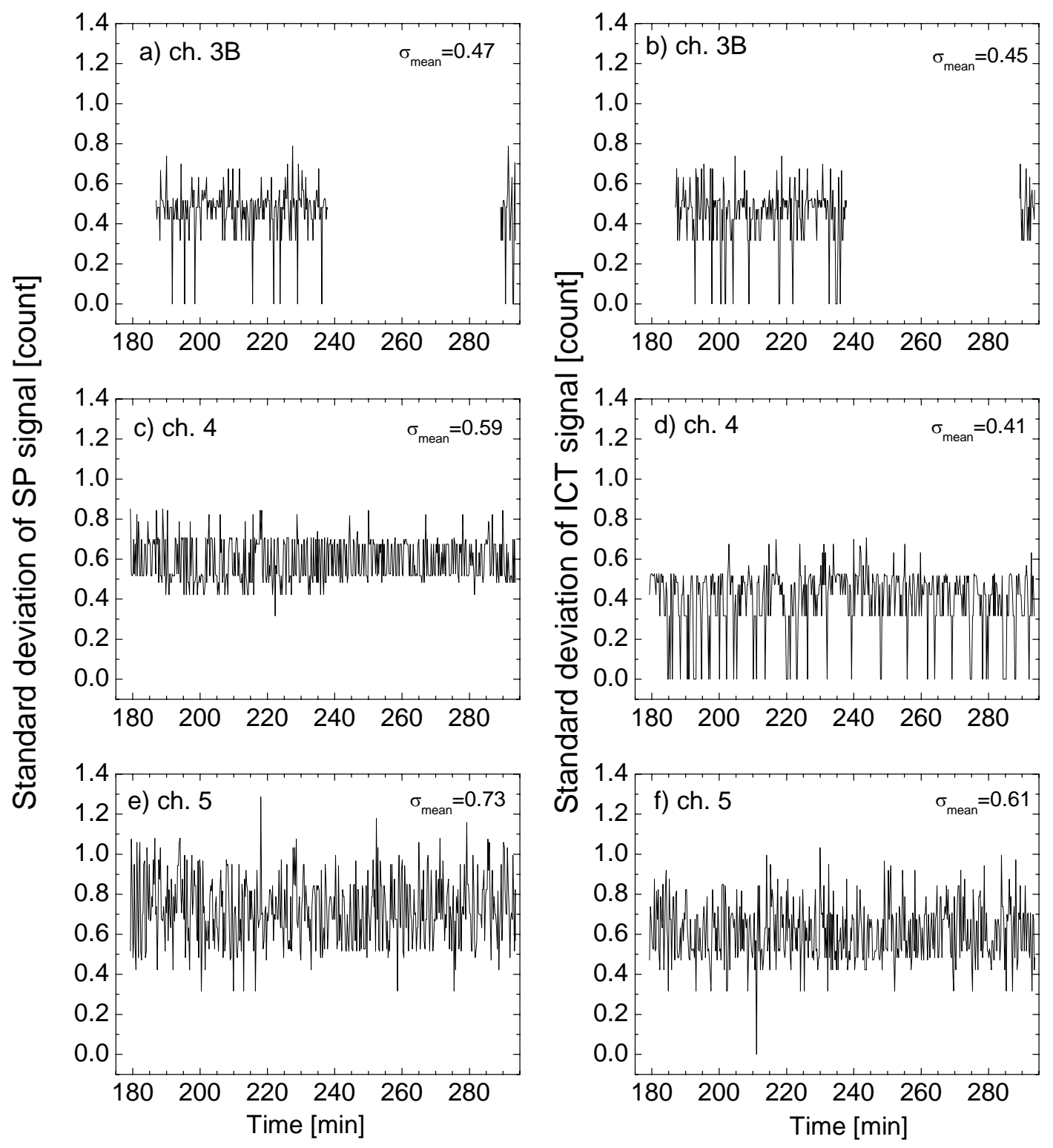

Figure 2 
Figure 3.
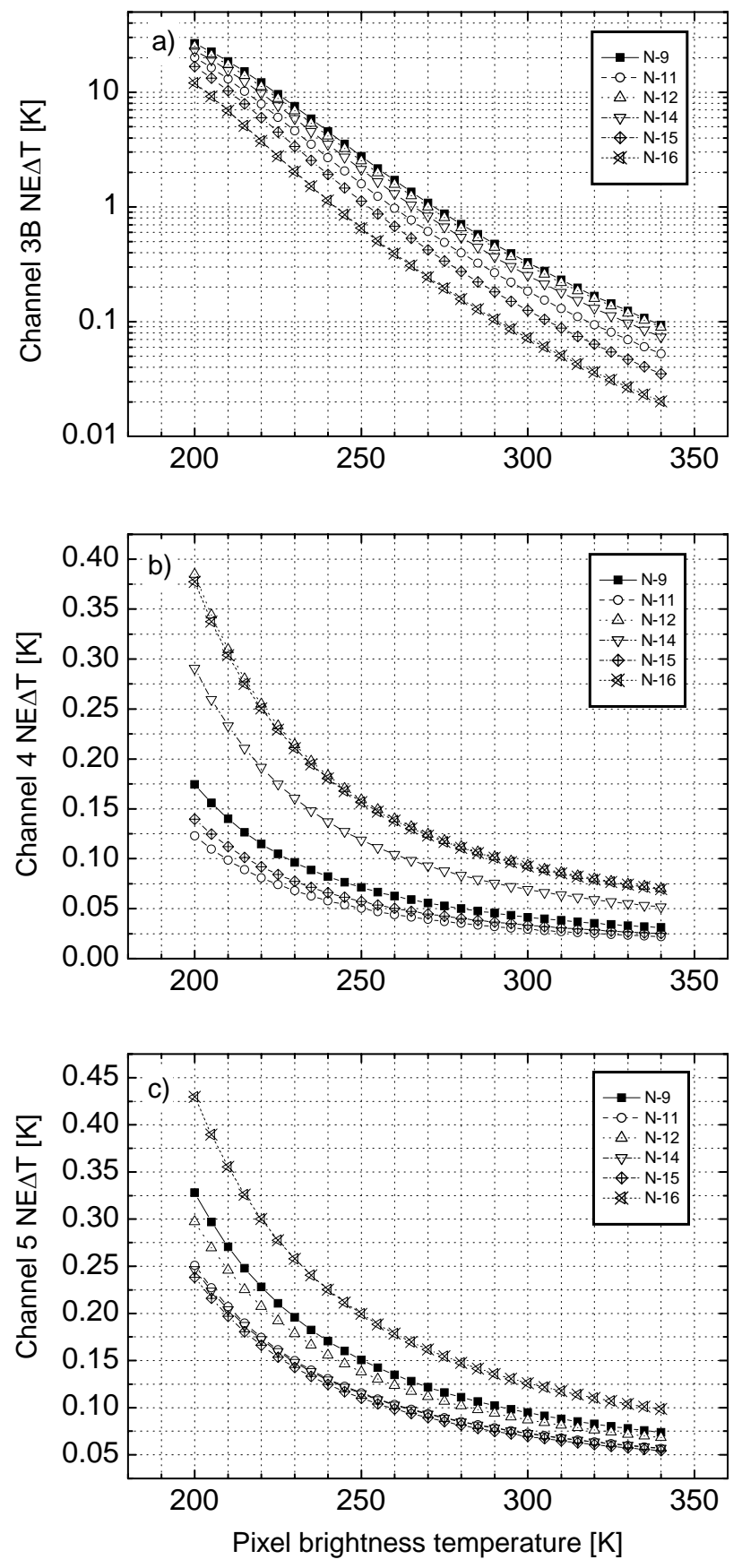

Figure 3 
Figure 4.
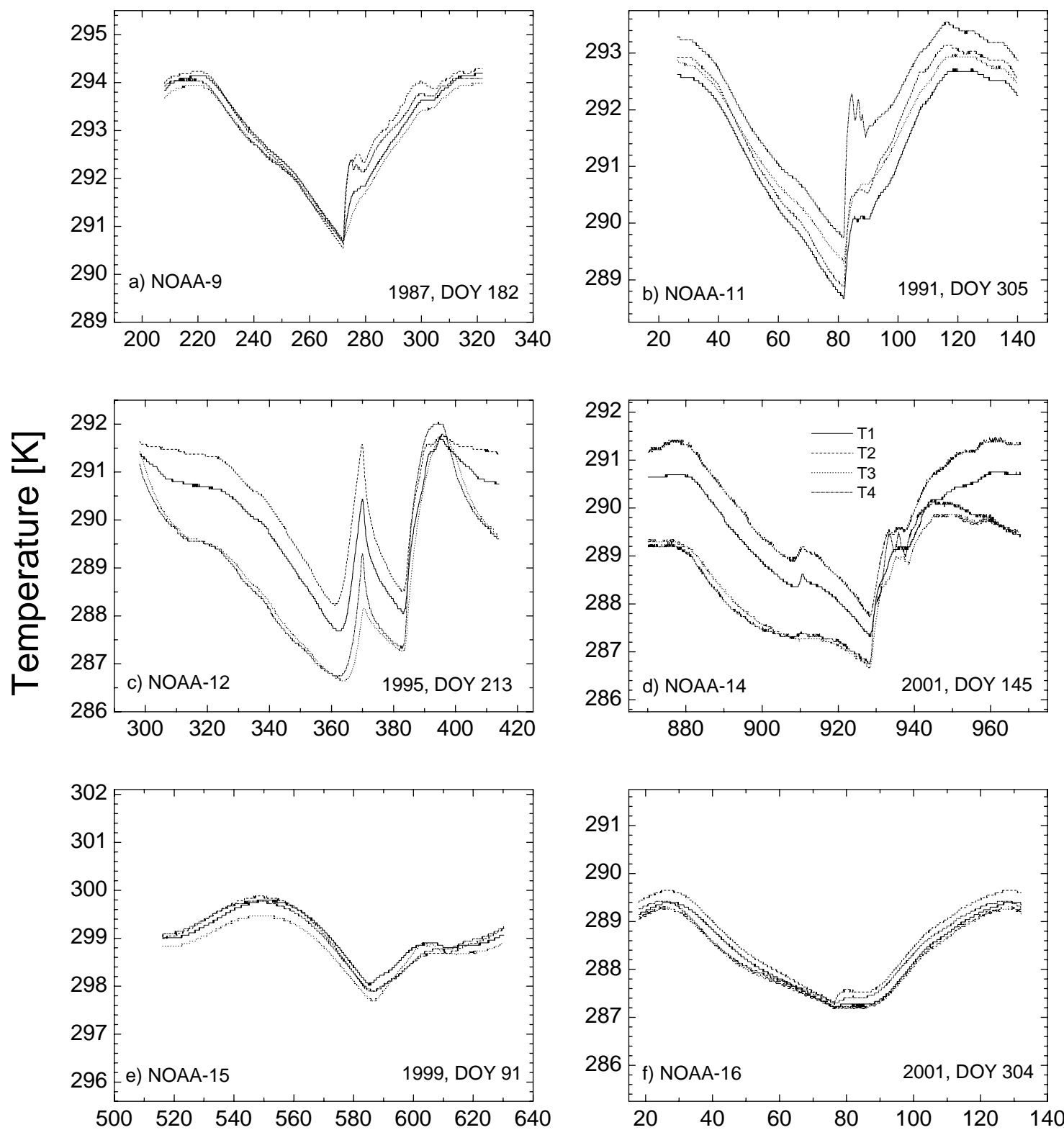

Time [min]

Figure 4 
Figure 5.
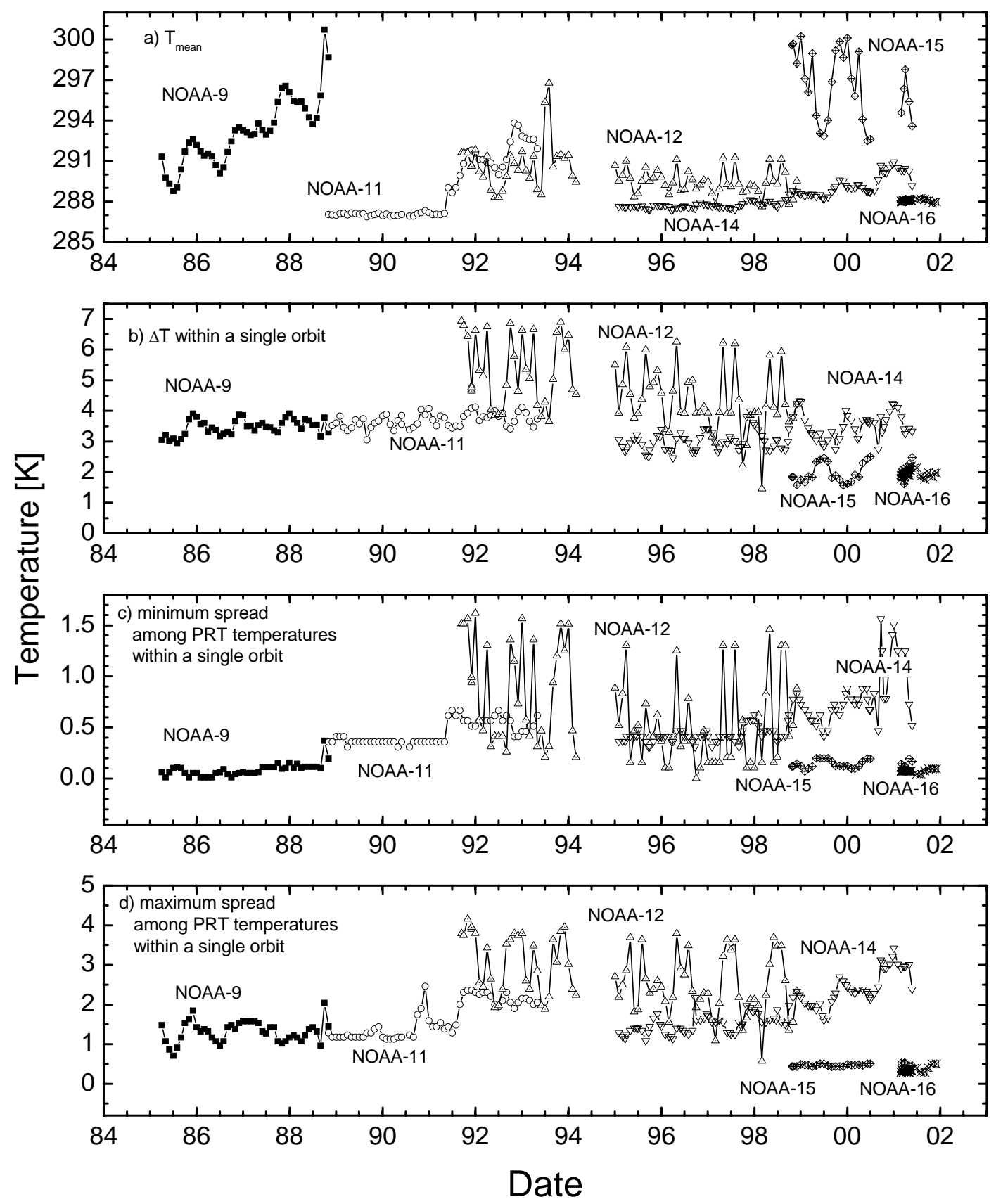

Figure 5 
Figure 6.

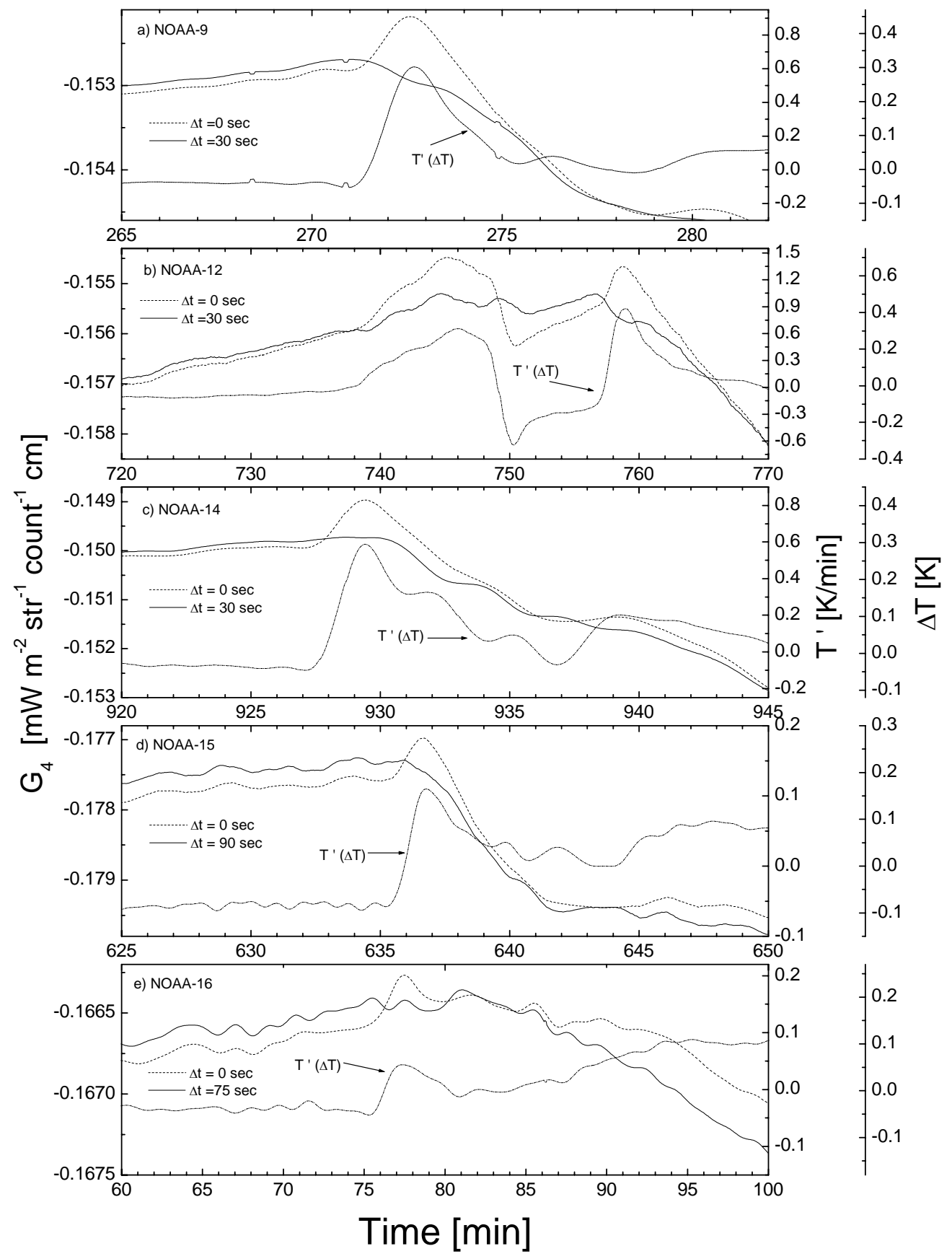

Figure 6 
Figure 7.

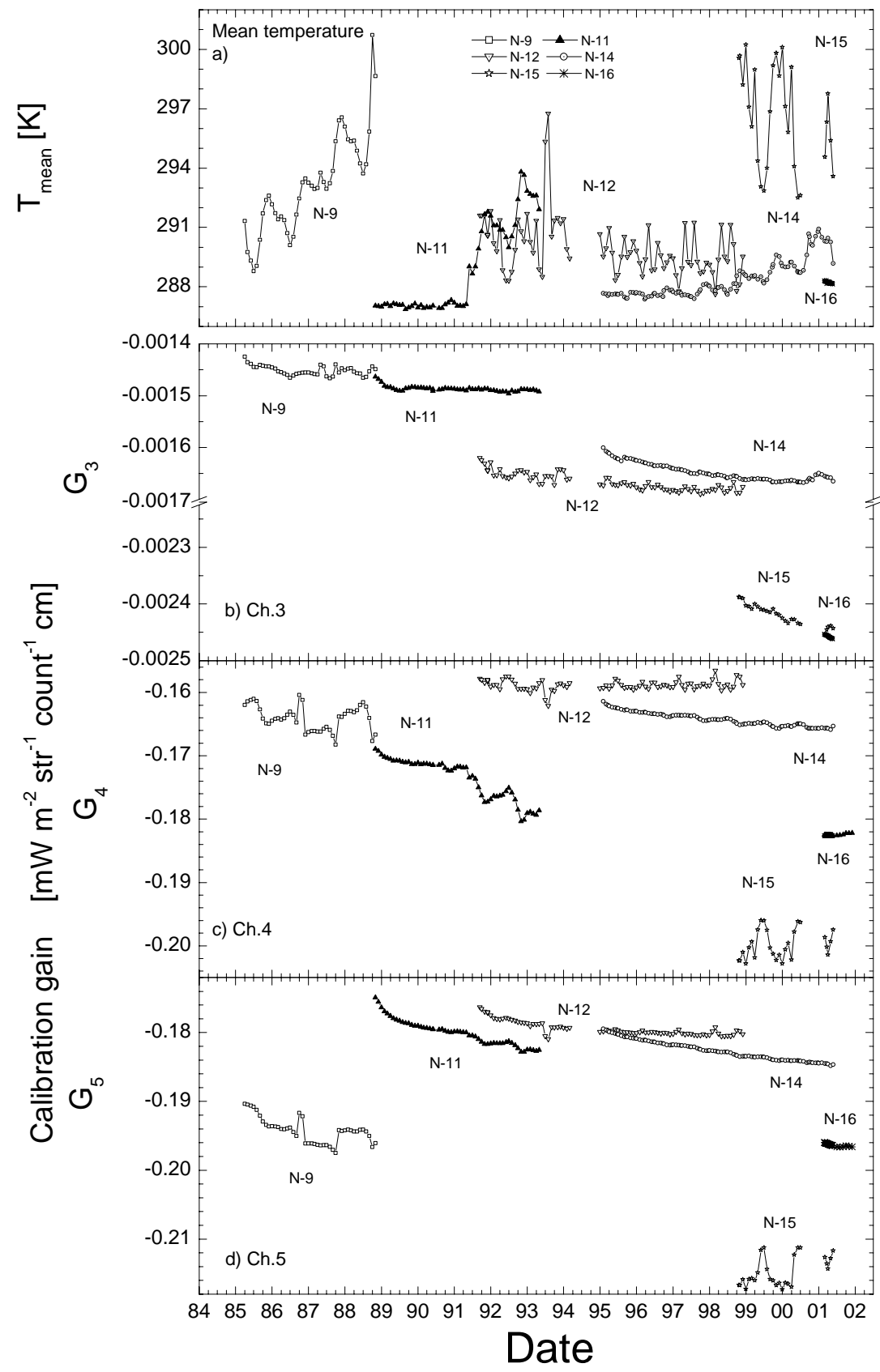

Figure 7 
Figure 8.

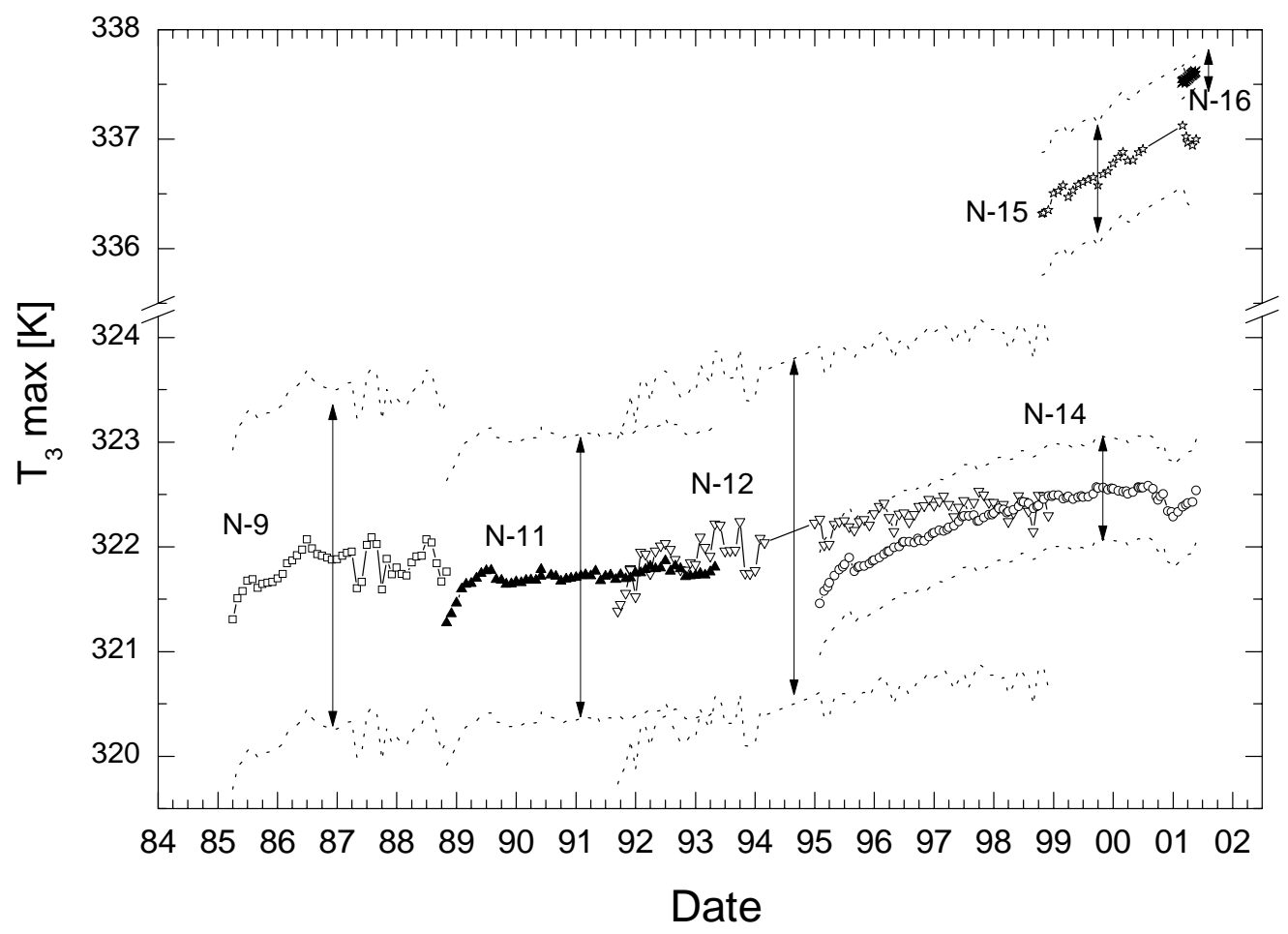

Figure 8 


\title{
Solar irradiance and effective brightness temperature for SW IR channels of AVHRR/NOAA and GOES imagers
}

\author{
ALEXANDER P. TRISHCHENKO \\ Canada Centre for Remote Sensing, Earth Sciences Sector, Natural Resources Canada, 588 \\ Booth Street, Ottawa, Ontario, Canada, K1A0Y7 \\ Tel 1-613-995 57 87; fax: 1-613-947 1385 \\ E-mail: trichtch@ccrs.nrcan.gc.ca
}

Submitted to

Journal of Atmospheric and Oceanic Technology

January 27, 2005

Revised July 22, 2005 


\begin{abstract}
Satellite observations in the shortwave infrared (SW IR) part of spectrum between $3.5 \mu \mathrm{m}$ and $4.0 \mu \mathrm{m}$ deliver critically important information for many applications. The satellite signal in this spectral band consists of solar reflected radiation and thermal radiation emitted by surface, clouds and atmosphere. Accurate retrievals require precise knowledge of solar irradiance values within channel bandwidth. The magnitude of solar irradiance for shortwave infrared channels (3.7 $\mu \mathrm{m}-3.9 \mu \mathrm{m})$ for the AVHRR/NOAA-7 to NOAA-18 and GOES-8 to GOES-12 is considered in this paper. Four recent solar reference spectra (Kurucz, 1997; Gueymard, 2004; ASTM, 2000; Wehrli, 1985) are analyzed to determine uncertainties in the knowledge of solar irradiance values for SW IR channels of listed sensors. Because thermal radiation is frequently converted to effective blackbody temperature for analysis, computations and calibration purpose, it is proposed here to express band-limited solar irradiance values in terms of brightness temperature as well. It is shown that band-limited solar irradiance for AVHRR radiometers expressed in terms of blackbody equivalent brightness temperature correspond to the range $355 \mathrm{~K}-360 \mathrm{~K}$, and vary around $345 \mathrm{~K}$ for SW IR channels of GOES imagers. The values of bandlimited solar irradiance and brightness temperatures are provided for various reference solar spectra. The relative differences in band-limited solar irradiance computed for considered reference solar spectra are between $0 \%$ and $2.5 \%$. Differences expressed in terms of brightness temperatures may reach $0.8 \mathrm{~K}$. The results for the ASTM and the Kurucz reference spectra agree within $0.1 \%$ relative difference. Parameters of linear fits relating effective brightness temperatures and spectral radiance equivalent temperatures are also determined for all sensors. They are required for precise radiance-temperature and temperature-radiance conversion through Planck's functions in the case of finite spectral response of real sensors.
\end{abstract}




\section{Introduction}

Satellite observations in the shortwave infrared (SW IR) spectral region between $3.5 \mu \mathrm{m}$ and $4.0 \mu \mathrm{m}$ are important for many applications, such as cloud retrievals, sea surface and land surface temperature determination, wild fire detection, albedo, emissivity, land cover mapping among others (Cracknell, 1997; Kidwell, 1998, Cihlar et al., 2004). The radiative energy observed by a satellite sensor in this spectral band during daytime conditions consists of solar reflected and thermal emitted components with comparable magnitudes. For a particular frequency $v$, the amount of reflected spectral radiative energy $R(v)$ is proportional to the pixel reflectance $\rho(v)$ and incoming solar radiation $S_{0}(v) \mu_{0}$ at the top-ofthe-atmosphere (TOA). Variable $\mu_{0}$ denotes the cosine of the solar zenith angle (SZA). The outgoing emitted thermal radiation $E(v)$ is proportional to the pixel emissivity $\varepsilon(v)$ and thermodynamic temperature $T$ of the emitting surface. Thus, to derive a pixel reflective $R(v)$ or emitted radiative energy $E(v)$, one needs to separate these components in the total signal $L(v)$. Thus, the reflected solar radiative energy is given by

$$
R(v)=L(v)-E(v)
$$

Due to the finite spectral response of the instrument, the monochromatic equation must be replaced by integration over the spectral interval of the spectral response function $f(v)$ to derive in-band values.

$$
\begin{aligned}
& L=\int_{v_{\min }}^{v_{\max }} f(v) L(v) d v, \\
& R=\int_{v_{\min }}^{v_{\max }} f(v) R(v) d v, \\
& E=\int_{v_{\min }}^{v_{\max }} f(v) E(v) d v,
\end{aligned}
$$


where $v_{\min }$ and $v_{\max }$ are the minimum and maximum wavenumbers, respectively, for the spectral response function. Equation (1) then becomes

$$
R=L-E
$$

It is a common practice to use brightness temperature for satellite measurements in the thermal infrared region. The standard units are reflectance or albedo for the satellite data in the wavelength region less than $2 \mu \mathrm{m}$. Although the SW IR region from 3.5 $\mu \mathrm{m}$ to $4.0 \mu \mathrm{m}$ encompasses a region where reflected and emitted components have similar magnitude; frequently, the satellite measurements in this region are also converted into brightness temperatures without concern for the solar reflected component. Another reason why it is convenient to use the brightness temperatures in the SW IR region from $3.5 \mu \mathrm{m}$ to $4.0 \mu \mathrm{m}$ is the onboard calibration, which is conducted by means of blackbody calibration targets of a known temperature (Cracknel, 1997; Trishchenko and Li, 2001; Trishchenko, 2002, Trishchenko et al., 2002a).

While the use of brightness temperature in the SW IR regions became a common practice, there was little attempt of systematic analysis of solar irradiance values and their magnitude and uncertainty in terms of effective brightness temperature for operational satellite sensors in this spectral region. Published results are fragmentary and not always consistent (Roger and Vermote, 1998).

The attempt is made in this paper to fill this gap and to provide recommendations how to parameterize the solar component in the SW IR region in terms of brightness temperature. This parameterization makes analysis and computations easier and physically more justified because similar physical terms or parameter units are compared for both solar reflected and thermal emitted components.

The finite width of the spectral response function of real sensors requires careful consideration to achieve precise results in temperature-radiance conversion using the monochromatic Planck's equation. The relationship between effective blackbody brightness temperature and spectral radiance equivalent blackbody brightness temperature is considered and analytical parameterizations for the difference 
between these temperatures are provided for a number of operational satellite sensors in the SW IR spectral region. This analysis updates the National Atmospheric and Oceanic Administration (NOAA) recommendations for radiance-temperature conversion for Advanced Very High Resolution Radiometers AVHHR/3 SW IR channels and introduces this technique for AVHRR/2 and AVHRR/1 types of sensors. The NOAA recommendations are available from the NOAA Polar Orbiter User's Guide (Kidwell, 1998; Goodrum et al., 2000).

The paper is structured as follows. Section 2 contains a general discussion about solar and thermal spectra. Section 3 analyzes the definition of effective brightness temperatures and spectral radiance equivalent brightness temperature for monochromatic and non-monochromatic (finite spectral response function) cases. Section 4 considers spectral response function features of SW IR channels of AVHRR/NOAA and Geostationary Operational Environmental Satellite (GOES) imagers. Section 5 discusses how to separate thermal and solar components in total SW signal. Section 6 presents an analysis of solar irradiance values and equivalent brightness temperatures for AVHRR and GOES imager SW channels. Section 7 analyzes the effect of solar zenith angle and pixel albedo on the magnitude of brightness temperature of the solar reflected component. Section 8 presents an analysis of the quantitative relationship between effective blackbody brightness temperature and spectral radiance equivalent brightness temperatures and provides the coefficients of a linear regression of the difference between these two temperatures.

\section{Solar spectrum}

Detailed knowledge of the solar spectrum is required to obtain the bad-limited solar irradiance $S_{0}$ and equivalent brightness temperatures $T_{S}$ of the radiation reaching the top-of-the-atmosphere (TOA). There are several solar spectra currently in use. The Calibration and Validation (CALVAL) 
Working Group of the Committee on Earth Observation Satellites (CEOS) (Ungar and Desnos, 2004) has recently recommended the solar spectrum of Thuillier et al. (2003). This spectrum, however, does not cover the spectral region beyond $2.4 \mu \mathrm{m}$. Four spectra that cover the entire range up to $4.0 \mu \mathrm{m}$ are those by Wehrli (1985), Kurucz (1997), American Society for Testing and Materials (ASTM) (2000) and recently published spectrum described by Gueymard (2004). The Kurucz spectrum is widely used by the atmospheric radiation modeling community through the MODTRAN atmospheric radiative transfer model. The Kurucz spectrum employed in our study was taken from MODTRAN 4 package (Berk et al., 1998). There are also some older solar spectra published prior to 1985 that cover the wavelength range greater than $2.5 \mu \mathrm{m}$ such as Labs and Neckel (1968), Neckel and Labs (1984), Thekaekara (1974) and some others. However, we will not use them here assuming that the recent data are of better accuracy.

Comprehensive intercomparisons of many spectra have been conducted by Thuillier et al. (2004) and Gueymard (2004). These analyses indicated that the commonly accepted value of the total solar irradiance (TSI) is $1366.1 \mathrm{Wm}^{-2}$ (Gueymard, 2004; Frölich, 2004), although there are still differences of a few percent level between various reference spectra at various wavenumbers. Since the Kurucz solar spectrum from MODTRAN 4 has slightly larger TSI value by $\sim 0.1 \%$, it was normalized in this paper by applying constant factor at each wavelength to get a TSI of $1366.1 \mathrm{Wm}^{-2}$. The recent measurements reported by the SORCE (SOlar Radiation and Climate Experiment) satellite project team for TSI (Kopp, 2005) indicate somewhat lower TSI values than mentioned by Gueymard, (2004) and Frölich (2004).

Table 1 provides a summary of solar irradiance for several typical wide spectral bands computed from five solar reference spectra. Spectral bands of $1 \mu \mathrm{m}$ width beyond $\lambda>1 \mu \mathrm{m}$ region were considered. In the wavelength region $\lambda<1 \mu \mathrm{m}$, three spectral bands were selected: $0.2 \mu \mathrm{m}$ to $0.4 \mu \mathrm{m}$, $0.4 \mu \mathrm{m}$ to $0.7 \mu \mathrm{m}$ (Photosynthetically Active Radiation PAR) and $0.7 \mu \mathrm{m}$ to $1 \mu \mathrm{m}$. Two broadband intervals: total shortwave (SW) $(0.2 \mu \mathrm{m}$ to $5.0 \mu \mathrm{m})$ and total (shortwave +longwave) $(<100 \mu \mathrm{m})$ were 
also included. The relative difference for each spectral band is plotted in Figure 1 for four reference spectra: Gueymard (2004), ASTM (2000), Wehrli (1985) and Thuillier et al. (2003). The comparison for the last spectrum is limited to the range of $0.2 \mu \mathrm{m}$ to $2 \mu \mathrm{m}$. Relative differences are defined with respect to the MODTRAN 4 Kurucz solar spectrum (Kurucz, 1997) normalized as described above. In the shortwave region, relative differences between the five spectra vary from $-6 \%$ to $+2.5 \%$. In the $3 \mu \mathrm{m}$ to $4 \mu \mathrm{m}$ spectral region, the ASTM and Kurucz spectra are very close to each other. The Gueymard spectrum is on average $\sim 1.75 \%$ lower and the Wehrli spectrum about 3.2\% lower than the Kurucz one. The relative differences in the region $\lambda>5 \mu \mathrm{m}$ are from nearly $-10 \%$ to $+1.2 \%$. Although, they look quite large, due to the overall small magnitude of the solar reflected component relative to the terrestrial thermal emitted component in the spectral region above $5 \mu \mathrm{m}$, this discrepancy is of less concern. The differences in the $0.2 \mu \mathrm{m}$ to $0.4 \mu \mathrm{m}$ and $1 \mu \mathrm{m}$ to $2 \mu \mathrm{m}$ and $2 \mu \mathrm{m}$ to $3 \mu \mathrm{m}$ spectral regions are of much greater concern. These relative differences range from $-1 \%$ to $-5.3 \%$ in the $0.2 \mu \mathrm{m}$ to $0.4 \mu \mathrm{m}$ spectral band. The relative differences in the PAR spectral band are within $\pm 0.5 \%$. In the $0.7 \mu \mathrm{m}$ to $1 \mu \mathrm{m}$ region, the differences are from $-0.1 \%$ to $+1 \%$. Relative differences vary from $+1.3 \%$ to $2.8 \%$ in $1 \mu \mathrm{m}-2 \mu \mathrm{m}$ and from $-0.1 \%$ to $-5.3 \%$ in $2 \mu \mathrm{m}-3 \mu \mathrm{m}$ region. Such a large disagreement between reference solar spectra may have quite important consequences for many remote sensing applications related to aerosol, cloud and land parameter retrievals.

\section{Effective brightness temperature of the solar radiation}

Another way of assessing the amount of energy in the solar spectrum is to convert the spectral radiance into equivalent brightness temperatures. For monochromatic radiation, this is achieved by the simple inversion of Planck’s formula 


$$
B(v, T)=\frac{c_{1} v^{3}}{\exp \left(\frac{c_{2} v}{T}\right)-1},
$$

where

$c_{1}=1.1910427 \times 10^{-8}\left[\mathrm{~W} \mathrm{~m} \mathrm{~m}^{-2} \mathrm{sr}^{-1} / \mathrm{cm}^{-4}\right]$ is the $1^{\text {st }}$ radiation constant for spectral radiance, and

$c_{2}=1.4387752[\mathrm{~cm} \mathrm{~K}]$ is the $2^{\text {nd }}$ radiation constant for spectral radiance.

By inverting this formula, temperature $T$ can be easily obtained as

$$
T=B^{-1}(v, E)=\frac{c_{2} v}{\ln \left(\frac{1+c_{1} v^{3}}{E}\right)},
$$

where $E$ is the spectral radiance.

The solar spectrum in terms of brightness temperature is shown in Figure 2. Results are shown for Kurucz solar spectrum normalized to $1366.1 \mathrm{~W} \mathrm{~m}^{-2}$ as explained in Section 2 above. Two types of curves are plotted. Black curves correspond to the Sun’s brightness temperature at TOA. Red curves correspond to the brightness temperatures of the Sun's surface computed as

$$
T=B^{-1}(v, E)=\frac{c_{2} v}{\ln \left(\frac{1+c_{1} v^{3}}{\left[\frac{R_{E-S}}{r_{S}}\right]^{2} E},\right.},
$$

where $r_{S}$ is the radius of the sun, equal to $6.96 \times 10^{5} \mathrm{~km}$, and $R_{E-S}$ is the average distance between Earth and the Sun, i.e. 1 astronomical unit (AU) equal to $1.4959787 \times 10^{8} \mathrm{~km}$. Figure 2 a shows the entire spectral range. Figure $2 \mathrm{~b}$ shows the details in the SW IR part of the solar spectrum from $2.75 \mu \mathrm{m}$ to $4.5 \mu \mathrm{m}$. The temperatures computed using Equation (5) are between $5000 \mathrm{~K}$ and $6000 \mathrm{~K}$. The typical brightness temperatures in the vicinity of $3.75 \mu \mathrm{m}$ at the TOA level are around $350 \mathrm{~K}$ to $360 \mathrm{~K}$. 
The relationship between radiance and brightness temperature for the non-monochromatic case with an arbitrary spectral distribution of the radiance $L(v)$ and spectral response function $f(v)$ is defined by the following expression

$$
L=\int_{v_{\min }}^{v_{\max }} f(v) L(v) d v=\int_{v_{\min }}^{v_{\max }} f(v) B\left(v, T_{\text {eff }}\right) d v,
$$

where $T_{\text {eff }}$ is the effective brightness temperature of the blackbody radiance field. For the entire unfiltered spectrum (i.e. $f(v)=1$ ) expression (5) is reduced to Stefan-Boltzmann's law

$$
L=\sigma T_{\text {eff }}^{4}
$$

where $\sigma=5.6703 \times 10^{-8} \mathrm{Wm}^{-2} \mathrm{~K}^{-4}$.

The values of effective brightness temperature for the spectral bands listed above are given in Table 2. Table 2 contains the same spectral bands as Table 1 and provides effective brightness temperatures at the TOA and the Sun's surface computed according to Equation (6) with rectangular spectral response function. The effective brightness temperature for the entire solar spectrum at the Sun's surface is around $5778 \mathrm{~K}$. However, effective temperatures at the TOA are significantly different. Effective temperatures decrease from about $2230 \mathrm{~K}$ in the $0.2 \mu \mathrm{m}$ to $0.4 \mu \mathrm{m}$ region to around $300 \mathrm{~K}$ at $5 \mu \mathrm{m}$ and as low as $65 \mathrm{~K}$ at $10 \mu \mathrm{m}$. One can see that the TOA solar brightness temperatures in the SW IR region are of the order of magnitude of the temperature for typical terrestrial scenes.

Equation (6) for band integrated irradiances can be transformed into an equation for band average spectral irradiances by simple normalization,

$$
\bar{L}=\frac{\int_{v_{\min }}^{v_{\max }} f(v) L(v) d v}{\int_{v_{\min }}^{v_{\max }} f(v) d v}=\frac{\int_{v_{\min }}^{v_{\max }} f(v) B\left(v, T_{\text {eff }}\right) d v}{\int_{v_{\min }}^{v_{\max }} f(v) d v}=B\left(v_{c}, T^{*}\right) .
$$


Therefore, effective brightness temperature $T_{\text {eff }}$ is the temperature of a blackbody that emits an equivalent amount of radiant energy within a spectral band characterized by the spectral response function $f(v)$. In the case of the average spectral radiance $\bar{L}$ described by Equation (8), one needs to specify the central wavenumber value $v_{c}$ and temperature $T^{*}$ to compute this quantity from Planck's equation. One possible approach to solve (6) or (8) is to assume that

$$
T^{*}=T_{\text {eff }}
$$

Using Equation (9), one can derive the value for $v_{c}$ from Equations (4) and (8). This approach ensures that the same temperature value is used in Planck's function in all parts of Equation (8). However, this definition depends on the spectrum $L(v)$ and may lead to different $v_{c}$ for different spectra.

It is, therefore, more convenient to define a constant central wavenumber $v_{c}$ in a traditional sense as the first moment:

$$
v_{c}=\frac{\int_{v_{\min }}^{v_{\max }} f(v) v d v}{\int_{v_{\min }}^{v_{\max }} f(v) d v} .
$$

With this definition, the central wavenumber is defined by the shape and location of the spectral response function and is an instrument-related parameter. However, it is not guaranteed that $T^{*}$ is equal to $T_{\text {eff }}$. This issue will be discussed later in this paper.

\section{Spectral response functions for SWIR channels of AVHRR and GOES Imagers}

Although spectral response functions for the same instrument type (AVHRR or GOES Imager) are similar, they are not identical. Each sensor's spectral response needs to be treated separately, similar 
to optical channels (Trishchenko et al., 2002b). The instrument spectral response functions of the sensors analyzed in this study are shown in Figure 3. We considered several AVHRRs onboard of NOAA satellites NOAA-7 to -18 and imagers on GOES-8 to -12. The SW IR channels for AVHRR/1 and AVHRR/2 types of sensors (i.e. prior to NOAA-15) are called channel 3. The SW IR channels of the AVHRR/3 type instrument (NOAA-15 and follow on platforms) are called channel 3B, as there is a satellite programming option to receive channel 3A $(1.6 \mu \mathrm{m})$ or 3B $(3.75 \mu \mathrm{m})$ data. The term 3B is used here on for all AVHRR $3.7 \mu$ m channels for consistency.

The tables of spectral response functions used in this paper were taken from the NOAA websites http://www2.ncdc.noaa.gov/docs/podug/html/c1/sec1-4.htm, http://www2.ncdc.noaa.gov/docs/klm/html/d/app-d.htm and http://www.oso.noaa.gov/goes/goescalibration/goes-imager-srfs.htm and International Satellite Cloud Climatology Project (ISCCP) website (http://isccp.giss.nasa.gov/docs/response.html). The AVHRR instruments typically have a wider spectral response than the GOES imagers. On average, spectral response functions for the GOES Imager SW IR channels are shifted towards longer wavelengths. Altogether, there are four groups among SW IR channel of the AVHRR sensors (AVHRR/N-7,-9,-10,-11; AVHRR/N-8,-12,-14; AVHRR/N-15 and -16; AVHRR/N- 17 and -18). All SW IR channels for GOES imagers fall under the same generic group. The differences between central wavelengths of AVHRR instruments are small, although there are some differences between shapes of the spectral response functions. The central wavelengths for the AVHRR sensors are around $3.70 \mu \mathrm{m}$ to $3.75 \mu \mathrm{m}$, while GOES imagers have central wavelengths around $3.90 \mu \mathrm{m}$.

The central wavenumbers $v_{C}$ computed in this paper according to Eq. (10) are given in the Table 3. To compute $v_{C}$ we interpolated the spectral response function $f(v)$ to a regular grid in wavenumber space at a spectral resolution of $1 \mathrm{~cm}^{-1}$ and calculated the integrals as simple sums. 


$$
\int_{v_{\min }}^{v_{\max }} f(v) F(v) d v=\sum_{k=k_{\min }}^{k_{\max }} f(k) F(k)
$$

Note that the central wavenumbers computed according to Equations (10-11) and listed in Table 3 are not the same as those provided by NOAA for calibration and conversion between radiance and brightness temperature. We provide values computed as the $1^{\text {st }}$ order statistical moment, while NOAA provides numbers optimized for radiance-temperature conversion (Equation (4)) and calibration. The NOAA KLM User’s Guide (Goodrum et al., 2000) specified among other parameters the $1^{\text {st }}$ order central moments for AVHRR/3 channel 3B. They are very close to those computed in this paper and given in Table 3, except for AVHRR/NOAA-16 where the difference is slightly more than $1 \mathrm{~cm}^{-1}$ (2697.45 $\mathrm{cm}^{-1}$ in this paper versus $2698.61 \mathrm{~cm}^{-1}$ reported by NOAA). The reason for this discrepancy is not clear, but it may be related to reported spectral leak around $4.25 \mu \mathrm{m}$ in channel $3 \mathrm{~B}$ for this sensor.

\section{Separating solar and thermal components in SWIR channels}

The general expression for radiance at the TOA level measured by a satellite sensor in the SW IR for the monochromatic case can be written as follows

$$
L(v)=\rho(v)\left[\frac{R_{A U}}{R_{E-S}}\right]^{2} S_{0}(v) \mu_{0}+\varepsilon(v) B(v, T),
$$

where:

$\rho(v)$ is the pixel reflectance,

$S_{0}(v)$ is the solar irradiance,

$\mu_{0}$ is the cosine of the solar zenith angle (SZA),

$\varepsilon(v)$ is the pixel emissivity,

$B(v, T)$ is black-body radiation corresponding to pixel temperature $T$. 
Factor $\left[\frac{R_{A U}}{R_{E-S}}\right]^{2}$ accounts for the correction of solar radiation due to the changing distance between the Earth and the Sun, where $R_{A U}$ is the average distance between the Earth and the Sun, equal to $1 \mathrm{AU}$, and $R_{E-S}$ is the distance between the Earth and the Sun at any particular moment. It is assumed in Equation (12) that the solar irradiance $S_{0}(v)$ is provided for the nominal distance of $1 \mathrm{AU}$.

Assuming a radiative equilibrium for a particular pixel, one can relate reflectance and emissivity as

$$
\varepsilon(v)+\rho(v)=1
$$

For the non-monochromatic case, the integration of Equations (12) and (13) over a finite spectral interval is required

$$
\begin{gathered}
\int_{v_{\min }}^{v_{\max }} f(v) L(v) d v=\int_{v_{\min }}^{v_{\max }} f(v)\left[\rho(v)\left[\frac{R_{A U}}{R_{E-S}}\right]^{2} S_{0}(v) \mu_{0}+\varepsilon(v) B(v, T)\right] d v \\
\int_{v_{\min }}^{v_{\max }} f(v)[\varepsilon(v)+\rho(v)] d v=\int_{v_{\min }}^{v_{\max }} f(v) d v
\end{gathered}
$$

The normalization by $\int_{v_{\min }}^{v_{\max }} f(v) d v$ reduces the above equations to expressions for band average quantities

$$
\begin{gathered}
\bar{L}=\bar{\rho}\left[\frac{R_{A U}}{R_{E-S}}\right]^{2} \bar{S}_{0} \mu_{0}+\bar{\varepsilon} \bar{B}(T) \quad \text { and } \\
\bar{\varepsilon}+\bar{\rho}=1
\end{gathered}
$$

where

$$
\bar{S}_{0}=\frac{\int_{v_{\min }}^{v_{\max }} f(v) S(v) d v}{\int_{v_{\min }}^{v_{\max }} f(v) d v},
$$

and we assume that $\rho(v)$ and $\varepsilon(v)$ vary slowly so that 


$$
\begin{aligned}
& \rho(v) \approx \text { const }=\bar{\rho} \quad \text { and } \\
& \varepsilon(v) \approx \text { const }=\bar{\varepsilon} .
\end{aligned}
$$

The average value for the blackbody function $\bar{B}(T)$ is defined as

$$
\bar{B}(T)=\frac{\int_{v_{\min }}^{v_{\max }} f(v) B(v, T) d v}{\int_{v_{\min }}^{v_{\max }} f(v) d v}
$$

If $\rho(v)$ and $\varepsilon(v)$ vary significantly over the spectral response function interval, we then assume that

$$
\bar{\rho}=\frac{\int_{v_{\min }}^{v_{\max }} f(v) \rho(v) S_{0}(v) d v}{\bar{S}_{0} \int_{v_{\min }}^{v_{\max }} f(v) d v}
$$

and a similar expression for emissivity can be derived with the solar spectrum replaced by Planck’s function.

For the convenience and effectiveness of computations, all variables $\bar{L}$ and $\bar{S}_{0}$ in Equation (12'”) can be expressed through the Planck's function at the specified wavenumber $v_{c}$ as

$$
\bar{L}=B\left(v_{c}, T^{*}\right)=\bar{\rho}\left[\frac{R_{A U}}{R_{E-S}}\right]^{2} \bar{S}_{0} \mu_{0}+\bar{\varepsilon} B\left(v_{c}, T_{p i x}\right)=\bar{\rho}\left[\frac{R_{A U}}{R_{E-S}}\right]^{2} B\left(v_{c}, T_{\text {sun }}^{*}\right) \mu_{0}+\bar{\varepsilon} B\left(v_{c}, T_{p i x}\right)
$$

The solar irradiance in Equation (17), is replaced by its parameterization through Planck's function at temperature $T_{\text {sun }}^{*}$. Temperature $T_{p i x}$ is derived by inversion

$$
T_{p i x}=B^{-1}\left(v_{c}, \bar{B}(T)\right)
$$

The temperature $T_{p i x}$ is defined as the thermodynamic temperature of a pixel and can be approximated by the temperature $T_{I R}$ observed in the IR-window $11 \mu \mathrm{m}$ to $12 \mu \mathrm{m}$ region. The temperature $T^{*}$ is a 
pixel spectral equivalent brightness temperature at $v_{c}$ derived from instruments counts in the result of calibration procedure and Equation (8). By substituting Equation (13’') into Equation (17), the expressions for pixel reflectance and emissivity can be derived as

$$
\begin{gathered}
\bar{\rho}=\frac{B\left(v_{c}, T^{*}\right)-B\left(v_{c}, T_{p i x}\right)}{\left[\frac{R_{A U}}{R_{E-S}}\right]^{2} \mu_{0} B\left(v_{c}, T_{\text {sun }}^{*}\right)-B\left(v_{c}, T_{p i x}\right)} \text { and } \\
\bar{\varepsilon}=\frac{B\left(v_{c}, T^{*}\right)-\left[\frac{R_{A U}}{R_{E-S}}\right]^{2} \mu_{0} B\left(v_{c}, T_{\text {sun }}^{*}\right)}{B\left(v_{c}, T_{p i x}\right)-\left[\frac{R_{A U}}{R_{E-S}}\right]^{2} \mu_{0} B\left(v_{c}, T_{\text {sun }}^{*}\right)}
\end{gathered}
$$

Equations (19) and (20) employ only blackbody functions, thus making analysis and interpretation of the relative contribution of solar and thermal components more expedient.

\section{Solar irradiance and effective brightness temperature for SWIR channels of AVHRR and}

\section{GOES Imagers}

The values of solar irradiance and brightness temperatures required in Equations (19) and (20) are provided in Table 3 for channel 3B of AVHRR/NOAA-7 to 18 and SW IR channels of GOES-8 to GOES -12 imagers. Computations were performed for several solar spectra: Kurucz (Kurucz, 1997; Berk et al., 1998).), Gueymard (2004), ASTM (2000) and Wehrli (1985). For each spectrum, Table 3 contains five parameters: central wavenumber $v_{C}$ in $\left[\mathrm{cm}^{-1}\right]$, band average spectral solar irradiance $\bar{S}_{0}$ in $\left[\mathrm{W} \mathrm{m}{ }^{-2} / \mathrm{cm}^{-1}\right]$, effective solar brightness temperature $\mathrm{T}_{\text {eff }}^{T O A}$ in $[\mathrm{K}]$ computed according to Equation (6), equivalent solar brightness temperature $\mathrm{T}^{*}$ in $[\mathrm{K}]$ computed according to Equation (8), effective 
brightness temperature at $1 \mathrm{AU} \mathrm{T}_{\text {eff }}^{A U}$ in [K] computed according to Equations (5) and (6). The difference between various reference spectra reflects the current level of uncertainty in the knowledge of the solar spectrum.

The absolute values and relative differences between solar irradiance values computed with different reference spectra are shown in Figures 4 a and 4b, where Figure 4a shows absolute values and Figure 4b shows relative differences computed with respect to Kurucz spectrum results. The results for the ASTM spectrum are very close to those of the Kurucz spectrum for all sensors considered. The relative differences between these two spectra are less than $0.1 \%$. Two other spectra show quite substantial relative differences between each other and with respect to the Kurucz and ASTM spectra. For the AVHRR channels, both spectra show close results and relative difference with respect to the Kurucz spectrum results varying from $-1.5 \%$ to almost $-2 \%$. The Gueymard spectrum shows larger differences for GOES imager SW IR channels ( up to -2.5\%) relative to the Kurucz spectrum results. The Wehrli spectrum reveals relative difference with respect to the Kurucz specturm of $-1.2 \%$.

Figure 5 shows solar irradiance values expressed in terms of brightness temperatures $T_{\text {eff }}^{\text {TOA }}$ computed according to Equation (6). Figure 5a displays absolute values of temperature. The temperatures for AVHRR channels 3B vary from about $354 \mathrm{~K}$ to values slightly greater than $360 \mathrm{~K}$. Temperatures for GOES imager SW IR channels are smaller. They are in the range of $342 \mathrm{~K}$ to $345 \mathrm{~K}$, i.e. by $10 \mathrm{~K}$ to $15 \mathrm{~K}$ smaller than temperatures for AVHRR channel 3B. The difference in brightness temperatures computed for different reference spectra taken with respect to the Kurucz data are presented in Figure 5b. The spread ranges from near $0 \mathrm{~K}$ to almost $-0.8 \mathrm{~K}$. The biggest difference is between the Kurucz and Gueymard spectra. This difference ranges from $-0.6 \mathrm{~K}$ to $-0.8 \mathrm{~K}$. The results for the Wehrli reference spectrum are close to those for the Gueymard spectrum for AVHRR channel 3B. The difference between the Wehrli and Kurucz reference spectra for GOES imager SW IR channels are by $0.2 \mathrm{~K}$ smaller ( $-0.4 \mathrm{~K}$ instead of $-0.6 \mathrm{~K}$ ). The Gueymard reference spectrum shows the largest 
differences for GOES SW IR channels (between $-0.7 \mathrm{~K}$ and $-0.8 \mathrm{~K}$ ). The Kurucz and ASTM spectra provide very similar results (within $0.01 \mathrm{~K}$ ) for all sensors considered.

\section{Solar zenith angle and pixel reflectance effect on magnitude of brightness temperature}

It is interesting to assess the effect of solar zenith angle and pixel reflectance on the magnitude of the effective brightness temperature of the solar reflected component. Equation (4) has to be modified as

$$
T\left(\mu_{0}\right)=B^{-1}\left(v, E=\rho S_{0} \mu_{0}\right)=\frac{c_{2} v}{\ln \left(\frac{1+c_{1} v^{3}}{\rho S_{0} \mu_{0}}\right)}
$$

The temperature $T$ of the solar reflected component is now a function of the cosine of the solar zenith angle $\mu_{0}$ and pixel reflectance $\rho$.

Figure 6 shows the dependence described by Equation (4') for 2 sensors a) AVHRR NOAA-16 ch.3B and b) GOES-10 SW IR imager channel. These two sensors represent maximum and minimum temperatures and cover the entire range of variability for all sensors considered. Results for seven pixel reflectance levels are shown: $100 \%, 50 \%, 20 \%, 10 \%, 5 \%, 1 \%$ and $0.1 \%$. Due to the logarithmic relationship between energy and temperature described by Equation (4'), the brightness temperature of the solar reflected component is quite high even for small $\rho$ and large solar zenith angles (low sun elevation). The brightness temperatures for the case of normal incidence and $100 \%$ reflection are between $360 \mathrm{~K}$ and $300 \mathrm{~K}$ for SZA up to $85^{\circ}$. The brightness temperature of the solar reflected component is larger than $240 \mathrm{~K}$ for most sensors up to $70^{\circ}-80^{\circ}$ of SZA even for pixel reflectance as small as 1\%. The results presented in Figure 6 indicate that the solar reflected component in Equation (1) and Equations (12) and (12') is potentially important for all observational conditions. The brightness temperature of the solar reflected component exceeds or is comparable to typical terrestrial and cloud 
temperatures for pixel albedo as low as $1 \%$ and almost the entire range of the solar zenith angles $\left(80^{\circ}\right.$ $\left.85^{\mathrm{O}}\right)$.

\section{Relationship between blackbody temperature $T_{\text {eff }}$ and radiance equivalent temperature $T^{*}$}

For computation purposes, it is easier to use Planck’s equation to compute pixel radiance from pixel brightness temperature or from the blackbody thermodynamic temperature in the case of data calibration than to perform numerical integration according to Equation (6). However, this convenience comes at a price. The relationship between band integrated radiance and band average spectral radiance described by Equation (8) introduces new radiance equivalent brightness temperature $T^{*}$, which is in general not equal to the effective blackbody brightness temperature $T_{\text {eff }}$. This temperature also depends on the central wavenumber $v_{c}$.

The NOAA manuals for AVHRR data processing (Goodrum et al, 2000; Kidwell, 1998) for the sensors prior to AVHRR/NOAA-15 recommend using a variable central wavenumber depending on the brightness temperature range and assume an equivalence between $T_{\text {eff }}$ and $T^{*}$. For AVHHR/3 sensors onboard of NOAA-15 and higher, the NOAA Polar Orbiter User’s Guide recommends a simple linear relationship between the above temperatures and uses a constant central wavenumber across the entire range of temperatures (Goodrum et al., 2000). To take advantage of this latter approach, which provides a simple yet accurate analytic expression for data calibration and radiance-temperature conversion, we applied this method for all sensors considered in this paper. Due to differences in the definition of central wavenumber $v_{c}$ our coefficients may differ from those provided in the NOAA manuals. 
To find the relationship between temperatures $T_{\text {eff }}$ and $T^{*}$, Equation (8) was solved numerically at $1 \mathrm{~K}$ steps for the range of blackbody temperatures $T_{\text {eff }}$ from $150 \mathrm{~K}$ to $375 \mathrm{~K}$. The tables of $T_{\text {eff }}, T^{*}$ and $\Delta T=T_{\text {eff }}-T^{*}$ were then used to derive the fitting parameters $a$ and $b$ as

$$
\Delta T=T_{\text {eff }}-T^{*}=a+b T_{\text {eff }} .
$$

Examples of fitting results for GOES and AVHRR SW IR channels are presented in Figure 7. Fitting accuracy is better than $0.01 \mathrm{~K}$ for all sensors with the exception of AVHRR/NOAA-16 (Figure 7c). There is a strong nonlinearity in this case due to a spectral leak at $4.25 \mu \mathrm{m}$ mentioned earlier. Linear fitting for AVHRR/NOAA-16 channel 3B provides accuracy within $0.1 \mathrm{~K}$. To achieve better results, a $3^{\text {rd }}$ degree polynomial is recommended. Parameters $a$ and $b$ of the linear fit for all sensors are given $\mathrm{n}$ the Table 4. Special approximation for AVHRR/NOAA-16 as a $3^{\text {rd }}$ degree polynomial is also given in the Table 4. Difference $\Delta T$ for this special case is expressed as a function of $T_{\text {eff }}$ and $T^{*}$ for convenience of radiance to temperature direct and inverse conversion. The differences $\Delta T$ are negative for all cases, i.e. radiance equivalent temperature $T^{*}$ is greater than effective blackbody temperature $T_{\text {eff }}$.

\section{Summary}

Satellite observations in the SW IR part of spectrum ( $3 \mu \mathrm{m}-4 \mu \mathrm{m})$ are important for many applications related to remote sensing of the surface, atmosphere and cloudiness. The electromagnetic radiation detected in this part of spectrum consists of two components: reflected radiation coming from the Sun and radiation emitted by the Earth atmosphere, clouds and surface. Due to the different nature of these components, they need to be separated from each other to retrieve information pertinent to specific physical processes in the Earth climate system. To separate these components and to carry out accurate retrievals, one needs to know precisely the spectrum of the incoming solar radiation. This paper 
analyses the information about the SW IR solar spectrum and its representation in terms of equivalent brightness temperature in detail. Representation of the solar spectrum in terms of brightness temperature makes the analysis of emitted and reflected components more efficient and convenient.

Several recent solar reference spectra have been analyzed to determine the range of uncertainty in the knowledge of solar irradiance for the satellite SW IR channels for AVHRR/NOAA-7 to -18 and imagers on GOES-8 to -12. Solar irradiance values, effective brightness temperatures and spectral radiance equivalent temperatures are provided for these sensors. The sensor band average spectral irradiances vary from approximately $1.6010^{-2} \mathrm{Wm}^{-2} / \mathrm{cm}^{-1}$ to approximately $1.4110^{-2} \mathrm{Wm}^{-2} / \mathrm{cm}^{-1}$ depending on the sensor spectral response function and the solar reference spectrum. While there is a commonly accepted value of the total solar irradiance $\left(1366.1 \mathrm{Wm}^{-2}\right)$, the difference between various solar reference spectra might be quite significant for selected spectral bands. For the SW IR channel, this uncertainty amounts to approximately 2.5\%. In terms of brightness temperature, this difference can be as large as $0.8 \mathrm{~K}$. Among 4 solar reference spectra considered in this paper, the Kurucz and ASTM spectra are similar to each other within $0.1 \%$ relative difference. The Wehrli and Gueymard spectra give smaller solar irradiances (from $-1.2 \%$ to approximately $-2.5 \%$ ) than the Kurucz and ASTM spectra.

The analysis of the relative contribution of solar reflected component to the total TOA signal proves that during daytime conditions this component is important for almost the entire solar zenith angle range ( up to $85^{\circ}$ or more ) and pixel reflectances as small as $1 \%$ where the signal is comparable to thermal emission from cold clouds.

To perform precise direct and inverse radiance to temperature conversion using Planck’s blackbody function at a single wavenumber for a finite spectral response range, it is necessary to define the relationship between effective blackbody brightness temperature and spectral equivalent brightness temperature. It is shown that the difference between these temperatures can be efficiently parameterized as a linear function of either temperature. Coefficients of the linear functions are provided for all the 
above sensors. The accuracy of the linear fit is better than $0.01 \mathrm{~K}$ except AVHRR/NOAA-16 where it is within $0.1 \mathrm{~K}$. The larger error occurs due to a spectral leak at $4.25 \mu \mathrm{m}$ for AVHRR/NOAA-16 channel 3B. The $3^{\text {rd }}$ degree polynomials are derived for the case of AVHRR/NOAA-16 to provide accuracy better than $0.01 \mathrm{~K}$.

\section{Acknowledgements}

This work was conducted at the Canada Centre for Remote Sensing (CCRS), Earth Sciences Sector of the Department of Natural Resources Canada as part of the project J28 of the Program “Reducing Canada’s Vulnerability to Climate Change”. This work was partially supported by the Canadian Space Agency under the Government Related Initiative Program (GRIP) grant to the Canadian Centre for Remote Sensing and the US Department of Energy Atmospheric Radiation Measurement (ARM) Program grant No. DE-FG02-02ER63351. The author thanks Gunar Fedosejevs and Andrew Davidson for critical review of the manuscript at CCRS. 


\section{References}

ASTM International, 2000, Standard Solar Constant and Zero Air Mass Solar Spectral Irradiance Tables. E490-00. American Society for Testing and Materials (ASTM).

Berk, A., Bernstein, L. S., Anderson, G. P., Acharya, P. K., Robertson, D. C., Chetwynd, J. H., and Adler-Golden, S..M., 1998, MODTRAN Cloud and Multiple Scattering Upgrades with Application to AVIRIS, Remote Sensing of Environment , 65, 367- 375, 1998.

Cihlar, J., Latifovic, R., Chen, J., Trishchenko, A.P., Du, Y., Fedosejevs, G., Guindon, G., 2004, Systematic corrections of the AVHRR image composites for temporal studies. Remote Sensing of Environment, 89, 217-233.

Cracknell, A. P., 1997, The Advanced Very High Resolution Radiometer (AVHRR). Taylor \& Francis, London, 534 pp

Fröhlich, C., 2004, Solar irradiance variability. in Solar Variability and its Effect on the Earth's Atmosphere and Climate System, edited by J. M. Pap, P. Fox, C. Frohlich, H.S. Hudson, J. Kuhn, J. McCormack, G. North, W. Sprigg, S.T. Wu, American Geophysical Union, Washington, DC, 97-110.

Goodrum, G., Kidwell, K.B., Winston, W. (Eds), 2000, NOAA KLM User’s Guide. Revised. US Department of Commerce, NESDIS, NOAA, National Climatic Data Center, Satellite Data Services Division, Washington, DC, USA.[available at http://www2.ncdc.noaa.gov/docs/klm/index.htm]

Gueymard. C., 2004, The Sun's total and spectral irradiance for solar energy applications and solar radiation models. Solar Energy, 76, 423-453.

Kidwell, K. B. (Editor), 1998, NOAA Polar Orbiter Data User's Guide. US Department of Commerce, NESDIS, NOAA, National Climatic Data Centre, Satellite Data Services Division, Washington, D.C., USA.

Koop, G., 2005, SORCE. Total solar irradiance data. [Available at http://lasp.colorado.edu/sorce/tsi_data.html].

Kurucz, R.L., 1997, The solar irradiance by computation. [Available at http://cfaku5.cfa.harvard.edu/papers/irradiance/].

Labs, D., and Neckel, H., 1968, The radiation of the solar photosphere from $2000 \stackrel{0}{A}$ to $100 \mu \mathrm{m}$. Z.Astrophys, 69, 1-73. 
Neckel, H. and Labs, D., 1984, The solar radiation between 3300 and 12500 Angstroms, Solar Physics, Vol. 90, pp. 205-258.

Roger, J.C. and Vermote, E.F., 1998, A method to retrieve the reflectivity signature at $3.75 \mu \mathrm{m}$ from AVHRR data. Remote Sensing of Environment, 64, 103-114.

Thekaekara, M.P., 1974, Extraterrestrial solar spectrum, 3000-6100 page 114 of "The Extraterrestrial Solar Spectrum" A.J Drummond and M.P. Thekaekara, Eds. Institute of Environmental Sciences, Mount Prospect Illinois, 1973.

Thuillier, G., M. Hers, P. C. Simon, D. Labs, H. Mandel, D. Gillotay, and T. Foujols, 2003, The solar spectral irradiance from 200 to $2400 \mathrm{~nm}$ as measured by the SOLSPEC spectrometer from the ATLAS and EURECA missions. Solar Physics. 214, 1-22.

Thuillier, G., Floyd, L., Woods, T.N., Cebula, R., Hilsenrath, E., Herse, M., and Labs, L., 2004, Solar Irradiance Reference Spectra, in Solar Variability and its Effect on the Earth's Atmosphere and Climate System, edited by J. M. Pap, P. Fox, C. Frohlich, H.S. Hudson, J. Kuhn, J. McCormack, G. North, W. Sprigg, S.T. Wu, American Geophysical Union, Washington, DC, 171-194.

Trishchenko, A.P., and Li, Z., 2001, A method for the correction of AVHRR onboard IR calibration in the event of short-term radiative contamination. International Journal of Remote Sensing, 22, 3619-3624

Trishchenko, A.P., 2002, Removing unwanted fluctuations in the AVHRR thermal calibration data using robust techniques, Journal of Atmospheric and Oceanic Technology, 19, 1939-1954.

Trishchenko, A.P., Fedosejevs, G., Li, Z., and Cihlar, J., 2002a, Trends and uncertainties in thermal calibration of AVHRR radiometers onboard NOAA-9 to NOAA-16. Journal of Geophysical Research, 107(D24), 4778, doi:10.1029/2002JD002353. ACL17-1 -ACL17-13.

Trishchenko, A.P., Cihlar, J., and Z. Li, 2002b, Effects of spectral response function on the surface reflectance and NDVI measured with moderate resolution sensors, Remote Sensing of Environment, 81, 1-18.

Ungar, S.G., Desnos, Y-L., 2004, The Working Group on Calibration and Validation. CEOS Newsletter , 22, page 6. [available at http://www.ceos.org/pages/NL/CEOS_NL22.pdf].

Wehrli, C., 1985, Extraterrestrial solar spectrum, WRC Publication 615. Physikalisch-Meteorologisches Observatorium + World Radiation Center (PMO/WRC) Davos Dorf, Switzerland, July 1985. 


\section{Figure captions}

Figure 1. Relative differences between integrated solar irradiance for Gueymard (2004), ASTM(2000), Wehrli (1985) and Thuillier (2003) spectra and Kurucz (1997) spectrum.

Figure 2. Effective brightness temperature of the solar radiation at the TOA (black) and the Sun's surface (red).

a) entire solar spectrum in SW and thermal regions;

b) SW IR interval (2.75-4.5 $\mu \mathrm{m})$.

Scale plotted in black is for black lines; scale plotted in red is for red lines. Results are shown for Kurucz solar spectrum normalized to $1366.1 \mathrm{~W} \mathrm{~m}^{-2}$ as explained in Section 2 of the text.

Figure 3. Spectral response functions of SW IR channels for AVHRR and GOES imagers.

Figure 4. The values of solar irradiance (top) and their relative difference with respect to Kurucz spectrum (bottom) for various SW IR channels of AVHRR/NOAA and GOES imagers. Data are shown for Kurucz, Gueymard, ASTM and Wehrli reference spectra.

Figure 5. (a) The values of effective brightness temperature of the solar radiation at the TOA level for SW IR channels of AVHRR/NOAA and GOES imagers.

(b) The difference $\Delta T=T_{\text {eff }}^{\text {TOA }}-T_{\text {eff }, \text { Kurucz }}^{\text {TOA }}$ relative to Kurucz solar spectrum

Figure 6. The dependence of brightness temperature of solar reflected component $T_{\text {eff }}$ at the TOA on solar zenith angle. Various pixel albedo values ranging from $100 \%$ to $0.1 \%$ are shown. Due to the logarithmic relationship between energy and temperature in Equation (4'), the solar brightness temperature exceeds or is comparable to typical terrestrial and cloud temperatures for pixel albedo as low as $1 \%$ and almost the entire range of the solar zenith angles $\left(80^{\mathrm{O}}-85^{\mathrm{O}}\right)$.

a) AVHRR/NOAA-16 channel 3B with highest $T_{\text {eff }}$ among all sensors considered, b) GOES 10 SW IR channel with lowest $T_{\text {eff }}$. 
Figure 7. Fitting the difference $\Delta T=T_{\text {eff }}-T^{*}$ between effective blackbody temperature $T_{\text {eff }}$ and radiance equivalent temperature $T^{*}$ for GOES-8 imager, AVHRR/NOAA-9 and AVHRR/NOAA-16. Nonlinearity for AVHRR/NOAA-16 is due to a spectral leak around $4.25 \mu \mathrm{m}$. In all cases, except AVHRR NOAA-16, linear fitting provides accuracy better than $0.01 \mathrm{~K}$. Linear fit for AVHRR/NOAA-16 is accurate within $0.1 \mathrm{~K}$. 


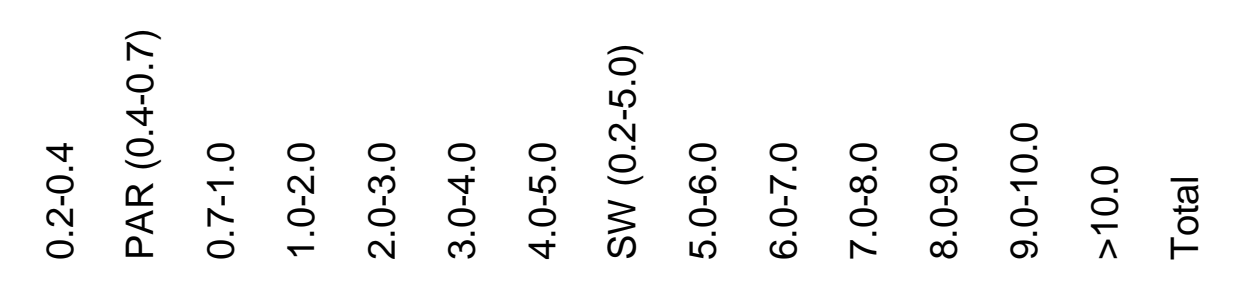

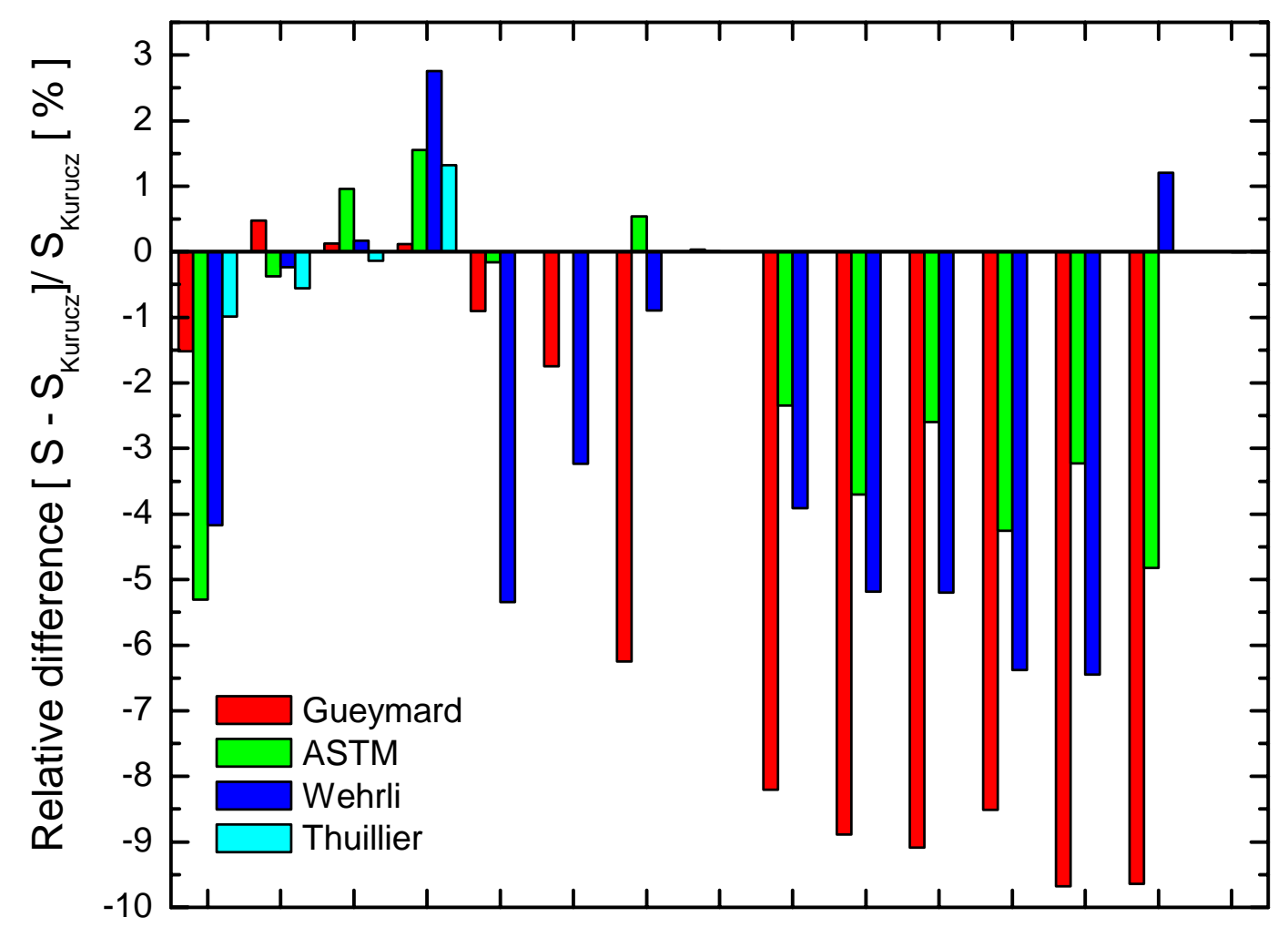

Figure 1. Relative differences between integrated solar irradiance for Gueymard (2004), ASTM(2000), Wehrli (1985) and Thuillier (2003) spectra and Kurucz (1997) spectrum. 


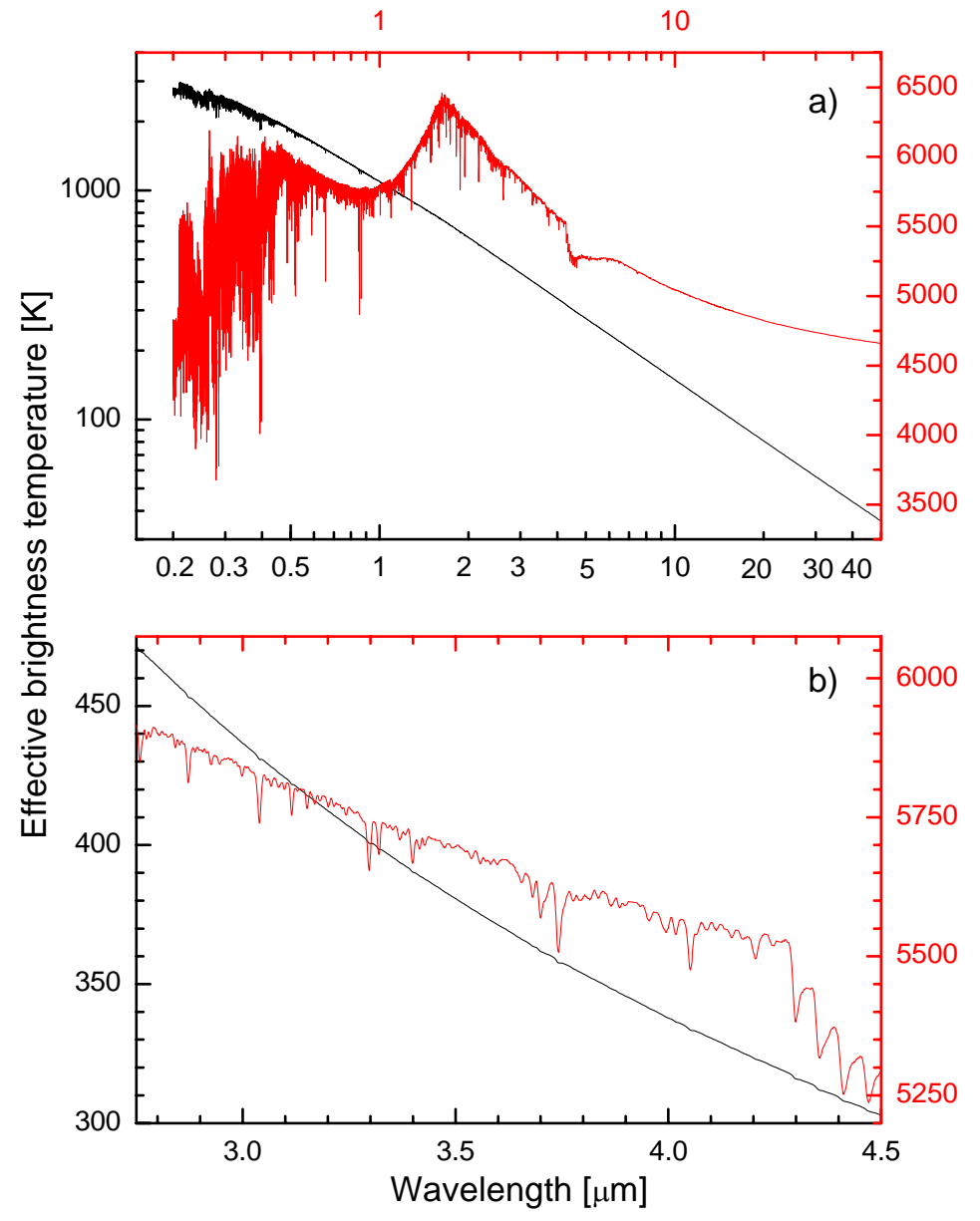

Figure 2. Effective brightness temperature of the solar radiation at the TOA (black) and the Sun's surface (red).

a) entire solar spectrum in SW and thermal regions;

b) SW IR interval $(2.75-4.5 \mu \mathrm{m})$.

Scale plotted in black is for black lines; scale plotted in red is for red lines. Results are shown for Kurucz solar spectrum normalized to $1366.1 \mathrm{~W} \mathrm{~m}^{-2}$ as explained in Section 2 of the text. 


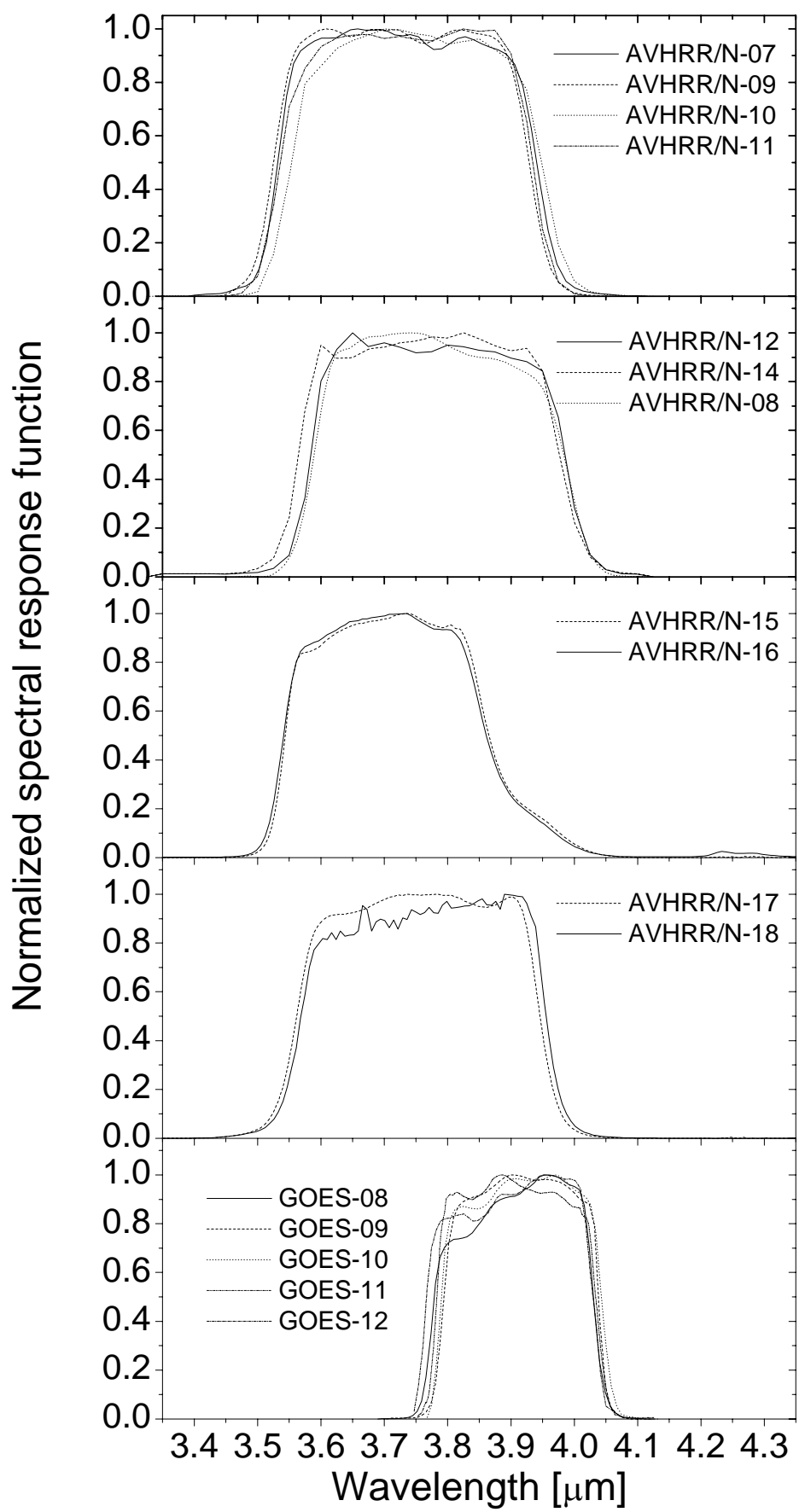

Figure 3. Spectral response functions of SW IR channels for AVHRR and GOES imagers. 


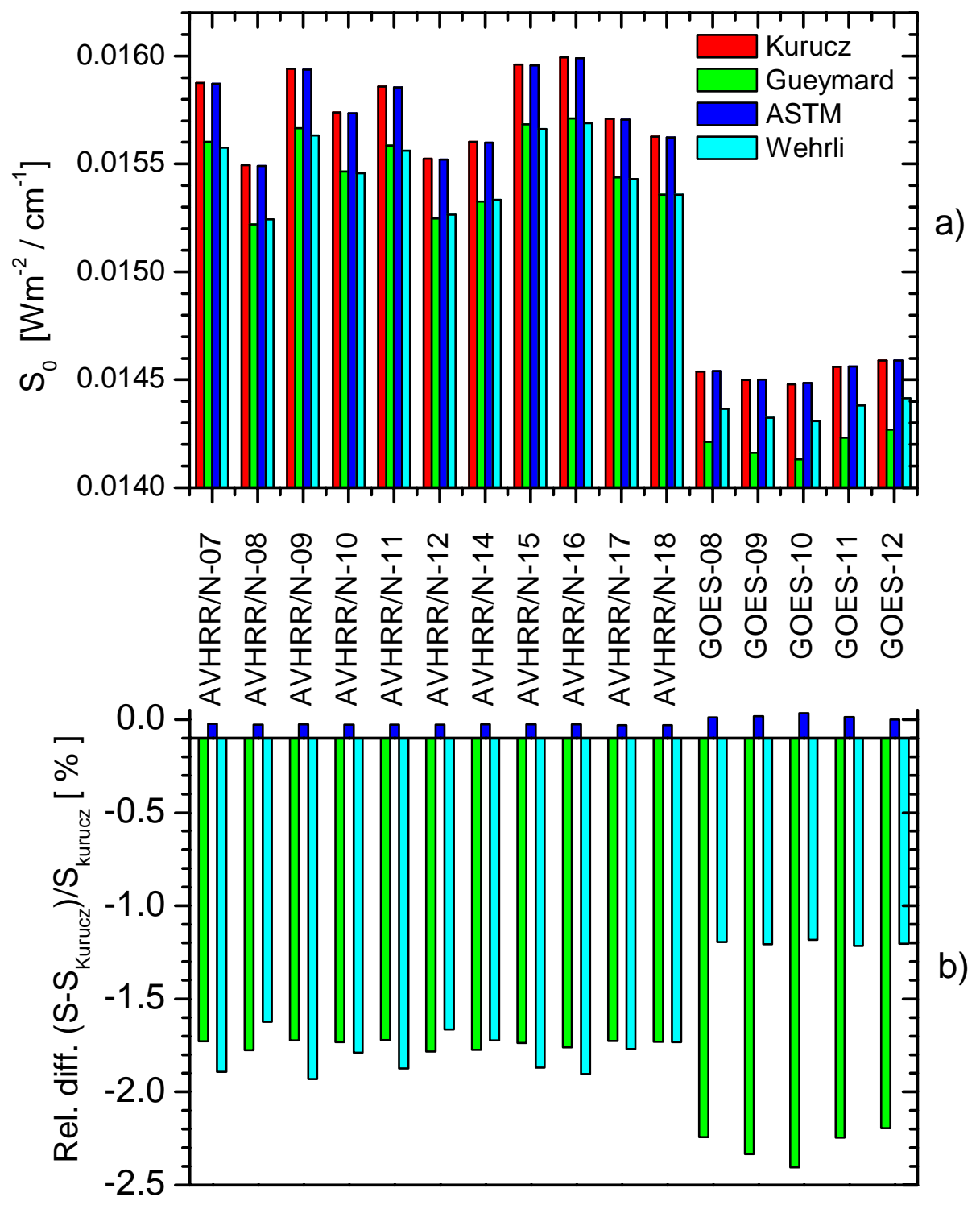

Figure 4. The values of solar irradiance (top) and their relative difference with respect to Kurucz spectrum (bottom) for various SW IR channels of AVHRR/NOAA and GOES imagers. Data are shown for Kurucz, Gueymard, ASTM and Wehrli spectra. 


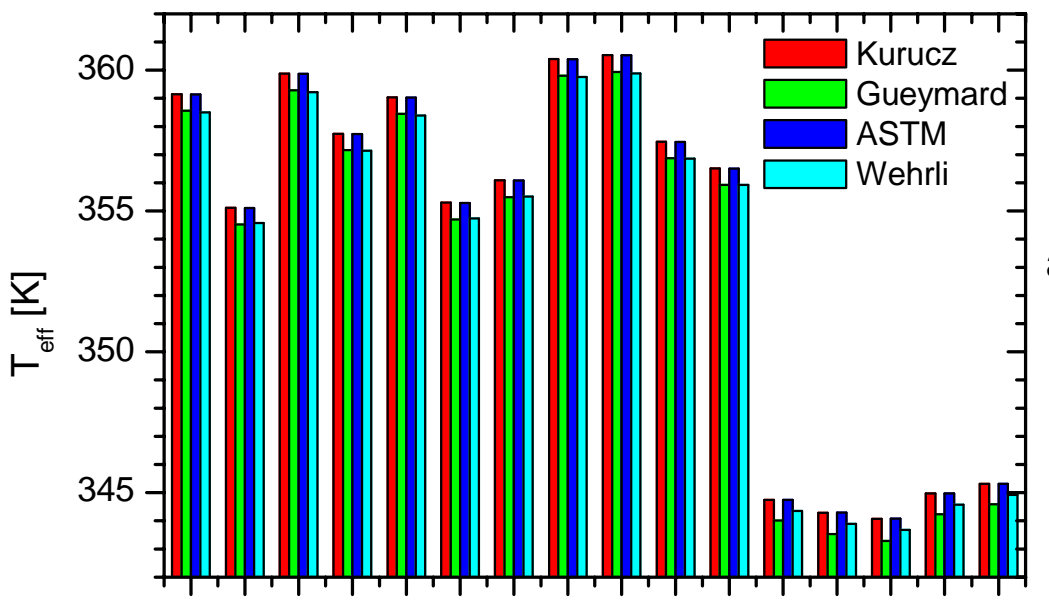

a)

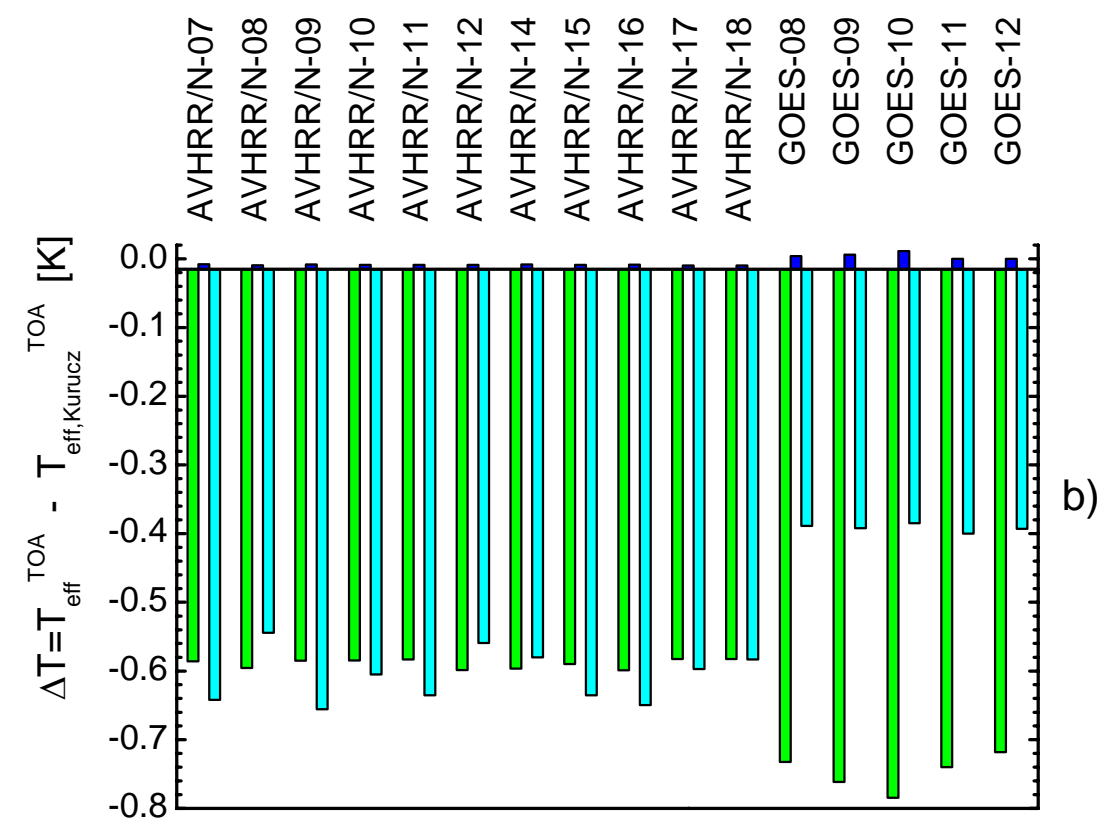

Figure 5. (a) The values of effective brightness temperature of the solar radiation at the TOA level for SW IR channels of AVHRR/NOAA and GOES imagers.

(b) The difference $\Delta T=T_{\text {eff }}^{\text {TOA }}-T_{\text {eff, Kurucz }}^{\text {TOA }}$ relative to Kurucz solar spectrum. 


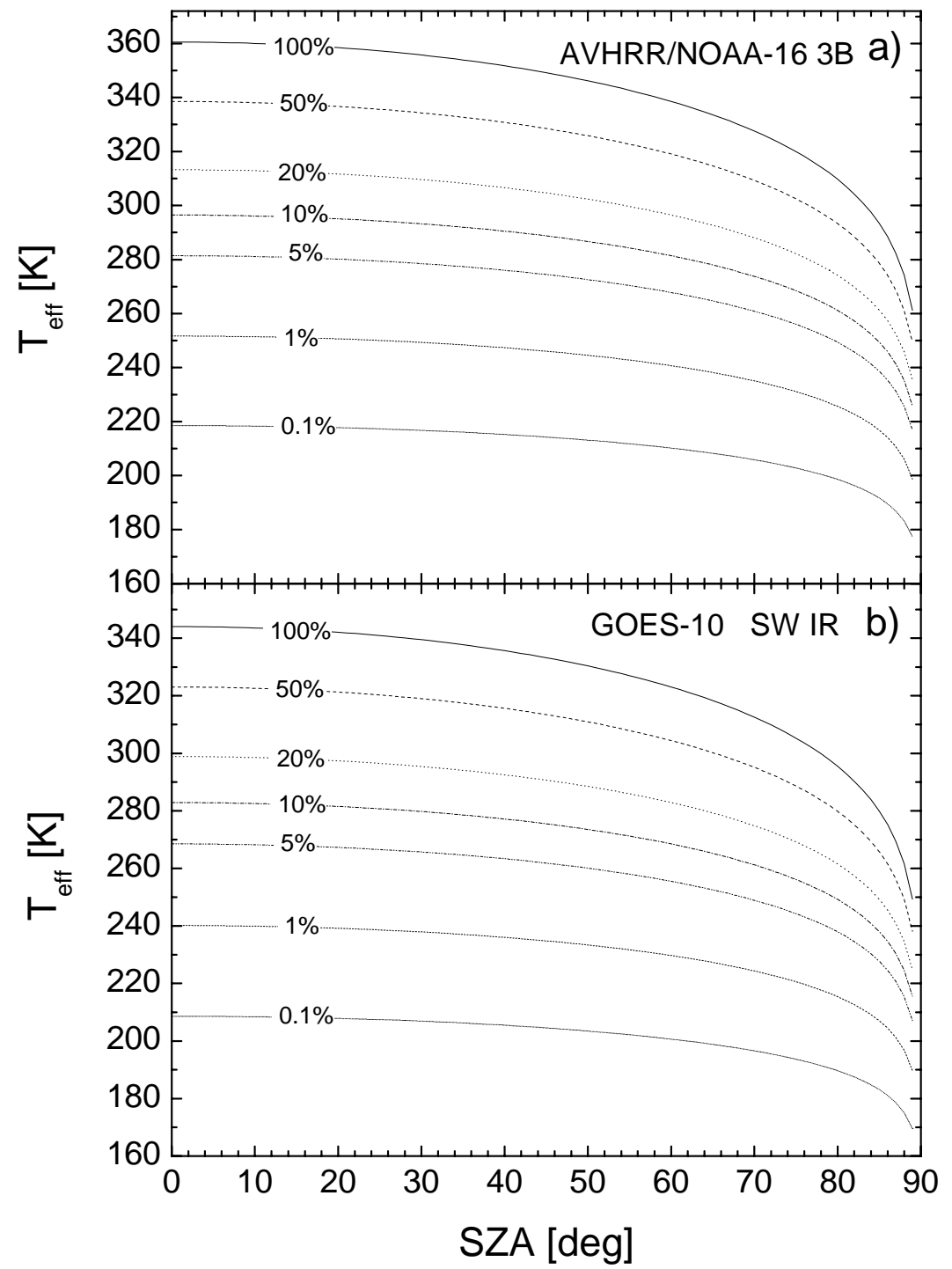

Figure 6. The dependence of brightness temperature of solar reflected component $T_{\text {eff }}$ at the TOA on solar zenith angle. Various pixel albedo values ranging from $100 \%$ to $0.1 \%$ are shown. Due to the logarithmic relationship between energy and temperature in Equation (4'), the solar brightness temperature exceeds or is comparable to typical terrestrial and cloud temperatures for pixel albedo as low as $1 \%$ and almost the entire range of the solar zenith angles $\left(80^{\circ}-85^{\circ}\right)$.

a) AVHRR/NOAA-16 channel 3B with highest $T_{\text {eff }}$ among all sensors considered, b) GOES 10 SW IR channel with lowest $T_{\text {eff }}$. 


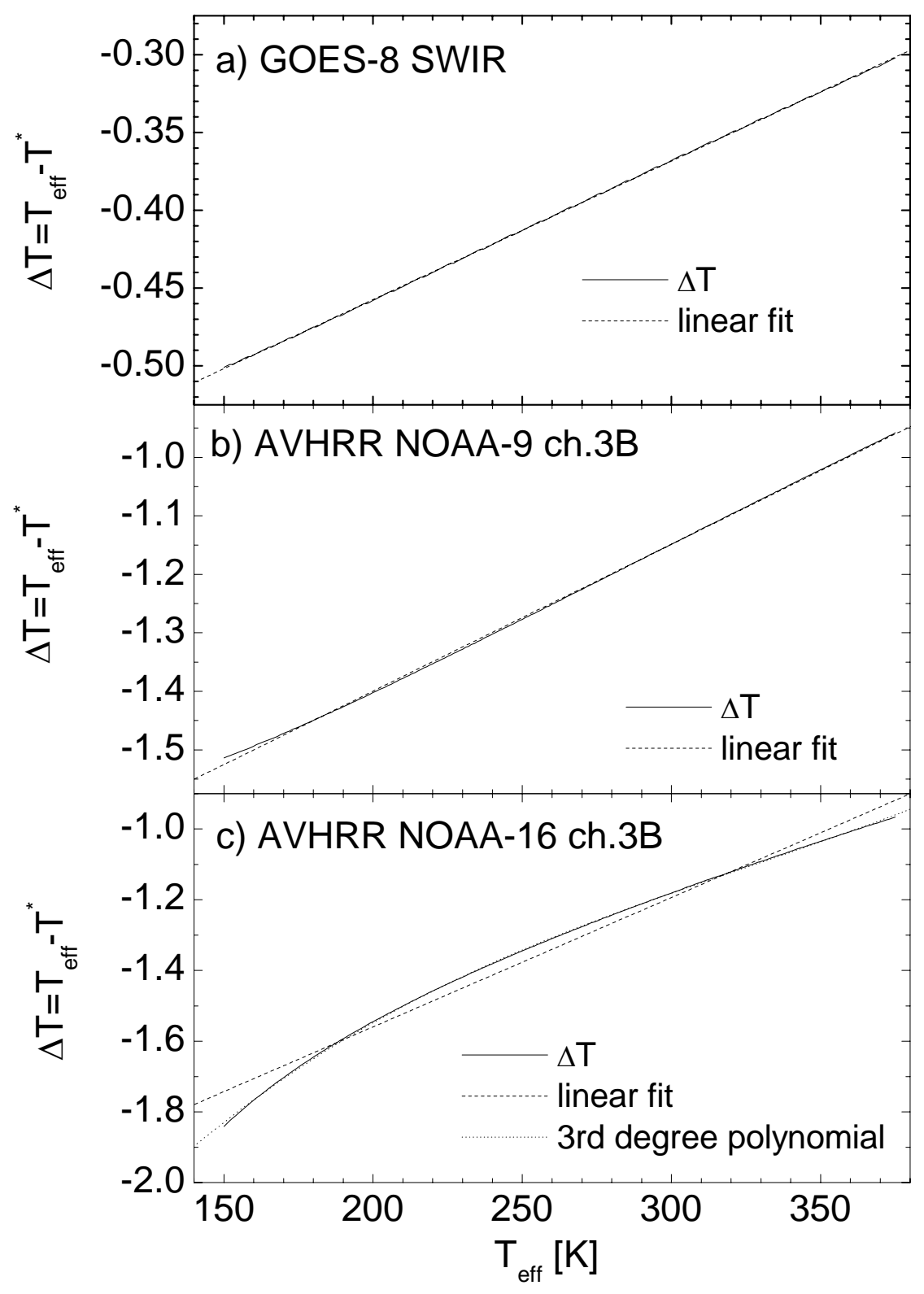

Figure 7. Fitting the difference $\Delta T=T_{\text {eff }}-T^{*}$ between effective blackbody temperature $T_{\text {eff }}$ and radiance equivalent temperature $T^{*}$ for GOES-8 imager, AVHRR/NOAA-9 and AVHRR/NOAA-16.

Nonlinearity for AVHRR/NOAA-16 is due to a spectral leak around $4.25 \mu \mathrm{m}$. In all cases, except AVHRR NOAA-16, linear fitting provides accuracy better than $0.01 \mathrm{~K}$. Linear fit for AVHRR/NOAA16 is accurate within $0.1 \mathrm{~K}$. 


\section{Tables}

Table 1. Comparison of the total solar energy in several bands for different reference spectra.

Table2. Solar spectral irradiance and effective brightness temperature for several reference solar spectra

Table 3. Solar irradiance and effective brightness temperature for SW IR channels of AVHRR/NOAA (3B) and GOES imagers.

Table 4. Coefficients of linear fit for temperature correction $\Delta T=T_{\text {eff }}-T^{*}=a+b T_{\text {eff }}$ 

Table 1. Comparison of the total solar energy in several bands for different reference spectra.

\begin{tabular}{|c|c|c|c|c|c|}
\hline $\begin{array}{l}\text { Spectral band } \\
{[\mu \mathrm{m}]}\end{array}$ & $\begin{array}{c}\text { Kurucz }^{*} \\
\mathrm{~W}\left[\mathrm{Wm}^{-2}\right]\end{array}$ & $\begin{array}{l}\text { Gueymard } \\
\mathrm{W}\left[\mathrm{Wm}^{-2}\right]\end{array}$ & $\begin{array}{c}\text { ASTM } \\
\mathrm{W}\left[\mathrm{Wm}^{-2}\right]\end{array}$ & $\begin{array}{c}\text { Wehrli } \\
\mathrm{W}\left[\mathrm{Wm}^{-2}\right]\end{array}$ & $\begin{array}{c}\text { Thuillier et al. } \\
\mathrm{W}\left[\mathrm{Wm}^{-2}\right]\end{array}$ \\
\hline $0.2-0.4$ & 112.48 & 110.77 & 106.51 & 107.79 & 111.37 .0 \\
\hline PAR (0.4-0.7) & 532.10 & 534.64 & 530.12 & 530.84 & 529.14 \\
\hline $0.7-1.0$ & 308.22 & 308.60 & 311.18 & 308.74 & 307.79 \\
\hline $1.0-2.0$ & 328.51 & 328.90 & 333.62 & 337.57 & 332.84 \\
\hline $2.0-3.0$ & 57.45 & 56.93 & 57.36 & 54.38 & - \\
\hline $3.0-4.0$ & 15.46 & 15.19 & 15.46 & 14.96 & - \\
\hline $4.0-5.0$ & 5.60 & 5.25 & 5.63 & 5.55 & - \\
\hline SW (0.2-5.0) & 1359.8 & 1360.3 & 1359.9 & 1359.8 & - \\
\hline $5.0-6.0$ & 2.56 & 2.35 & 2.50 & 2.46 & - \\
\hline $6.0-7.0$ & 1.35 & 1.23 & 1.30 & 1.28 & - \\
\hline $7.0-8.0$ & 0.77 & 0.70 & 0.75 & 0.73 & - \\
\hline $8.0-9.0$ & 0.47 & 0.43 & 0.45 & 0.44 & - \\
\hline $9.0-10.0$ & 0.31 & 0.28 & 0.30 & 0.29 & - \\
\hline$>10.0$ & 0.83 & 0.75 & 0.79 & 0.84 & - \\
\hline Total & 1366.1 & 1366.1 & 1366.1 & 1366.0 & - \\
\hline
\end{tabular}

* original Kurucz spectrum was normalized to get TSI $1366.1 \mathrm{Wm}^{-2}$ 
Table2. Solar spectral irradiance and effective brightness temperature for several solar reference spectra.

\begin{tabular}{|c|c|c|c|c|c|c|c|c|}
\hline & \multicolumn{2}{|c|}{ Kurucz* } & \multicolumn{2}{c|}{ Gueymard } & \multicolumn{2}{c|}{ ASTM } & \multicolumn{2}{c|}{ Wehrli } \\
\hline $\begin{array}{c}\text { Spectral band } \\
{[\mu \mathrm{m}]}\end{array}$ & $\mathrm{T}_{\text {eff }}^{\text {TOA }}$ & $\mathrm{T}_{\text {eff }}^{A U}$ & $\mathrm{~T}_{\text {eff }}^{T O A}$ & $\mathrm{~T}_{\text {eff }}^{A U}$ & $\mathrm{~T}_{\text {eff }}^{\text {TOA }}$ & $\mathrm{T}_{\text {eff }}^{A U}$ & $\mathrm{~T}_{\text {eff }}^{\text {TOA }}$ & $\mathrm{T}_{\text {eff }}^{A U}$ \\
{$[\mathrm{~K}]$} & {$[\mathrm{K}]$} & {$[\mathrm{K}]$} & {$[\mathrm{K}]$} & {$[\mathrm{K}]$} & {$[\mathrm{K}]$} & {$[\mathrm{K}]$} \\
\hline $0.2-0.4$ & 2234.7 & 5501.2 & 2232.7 & 5490.6 & 2227.7 & 5463.8 & 2229.2 & 5471.9 \\
\hline $0.4-0.7$ & 1631.0 & 5851.1 & 1631.6 & 5857.1 & 1630.6 & 5846.5 & 1630.7 & 5848.2 \\
\hline $0.7-1.0$ & 1220.5 & 5746.9 & 1220.6 & 5749.0 & 1221.4 & 5764.1 & 1220.6 & 5749.8 \\
\hline $1.0-2.0$ & 775.0 & 5990.4 & 775.1 & 5993.6 & 776.2 & 6032.5 & 777.0 & 6064.9 \\
\hline $2.0-3.0$ & 512.2 & 6039.7 & 511.8 & 6004.9 & 512.1 & 6033.8 & 509.6 & 5835.9 \\
\hline $3.0-4.0$ & 380.8 & 5708.4 & 380.2 & 5638.8 & 380.8 & 5707.5 & 379.6 & 5578.1 \\
\hline $4.0-5.0$ & 303.7 & 5384.2 & 301.8 & 5127.8 & 303.8 & 5402.6 & 303.4 & 5348.0 \\
\hline SW $(0.2-5.0)$ & 565.6 & 5779.1 & 565.6 & 5779.6 & 565.6 & 5779.1 & 565.6 & 5779.1 \\
\hline $5.0-6.0$ & 254.1 & 5262.6 & 252.1 & 4934.9 & 253.5 & 5170.9 & 253.1 & 5107.0 \\
\hline $6.0-7.0$ & 219.3 & 5239.0 & 217.2 & 4844.4 & 218.5 & 5083.2 & 218.1 & 5004.9 \\
\hline $7.0-8.0$ & 193.1 & 5171.5 & 191.3 & 4786.6 & 192.4 & 5028.9 & 192.0 & 4932.1 \\
\hline $8.0-9.0$ & 172.7 & 5110.5 & 171.2 & 4750.1 & 172.2 & 4995.8 & 171.8 & 4886.5 \\
\hline $9.0-10.0$ & 156.4 & 5061.0 & 155.1 & 4728.0 & 156.1 & 4976.6 & 155.5 & 4832.5 \\
\hline$>10.0$ & 65.7 & 4931.9 & 64.3 & 4513.2 & 65.1 & 4760.6 & 65.9 & 4998.3 \\
\hline Total & 394.2 & 5778.0 & 394.2 & 5778.0 & 394.2 & 5778.0 & 394.2 & 5777.9 \\
\hline
\end{tabular}


Table 3. Solar irradiance and effective brightness temperature for SW IR channels of AVHRR/NOAA (3B) and GOES imagers.

\begin{tabular}{|c|c|c|c|c|c|c|c|c|c|}
\hline \multirow[b]{2}{*}{ Sensor } & \multirow[b]{2}{*}{$v_{C}\left[\mathrm{~cm}^{-1}\right]$} & \multicolumn{4}{|c|}{ Kurucz } & \multicolumn{4}{|c|}{ Gueymard } \\
\hline & & $\bar{S}_{0}\left[\mathrm{~W} \mathrm{~m} \mathrm{~m}^{-2} / \mathrm{cm}^{-1}\right]$ & $\mathrm{T}_{\text {eff }}^{T O A}[\mathrm{~K}]$ & $\mathrm{T}^{*}[\mathrm{~K}]$ & $\mathrm{T}_{e f f}^{A U}[\mathrm{~K}]$ & $\bar{S}_{0}\left[\mathrm{~W} \mathrm{~m} \mathrm{~m}^{-2} / \mathrm{cm}^{-1}\right]$ & $\mathrm{T}_{\text {eff }}^{T O A}[\mathrm{~K}]$ & $\mathrm{T}^{*}[\mathrm{~K}]$ & $\mathrm{T}_{\text {eff }}^{A U}[\mathrm{~K}]$ \\
\hline AVHRR/N-07 & 2685.81 & 1.5877E-02 & 359.15 & 360.17 & 5621.1 & 1.5602E-02 & 358.56 & 359.59 & 5550.8 \\
\hline AVHRR/N-08 & 2651.33 & $1.5494 \mathrm{E}-02$ & 355.11 & 356.02 & 5607.1 & 1.5219E-02 & 354.52 & 355.43 & 5534.9 \\
\hline AVHRR/N-09 & 2691.64 & $1.5941 \mathrm{E}-02$ & 359.87 & 360.87 & 5623.2 & $1.5666 \mathrm{E}-02$ & 359.29 & 360.29 & 5553.2 \\
\hline AVHRR/N-10 & 2673.53 & $1.5739 \mathrm{E}-02$ & 357.75 & 358.69 & 5615.9 & $1.5466 \mathrm{E}-02$ & 357.16 & 358.11 & 5545.5 \\
\hline AVHRR/N-11 & 2684.31 & $1.5856 \mathrm{E}-02$ & 359.03 & 359.99 & 5620.3 & $1.5586 \mathrm{E}-02$ & 358.45 & 359.41 & 5550.3 \\
\hline AVHRR/N-12 & 2653.67 & 1.5524E-02 & 355.29 & 356.31 & 5609.0 & $1.5248 \mathrm{E}-02$ & 354.69 & 355.72 & 5536.5 \\
\hline AVHRR/N-14 & 2660.74 & 1.5603E-02 & 356.09 & 357.17 & 5612.1 & 1.5327E-02 & 355.49 & 356.57 & 5540.0 \\
\hline AVHRR/N-15 & 2694.64 & 1.5960E-02 & 360.39 & 361.20 & 5621.2 & 1.5683E-02 & 359.80 & 360.61 & 5550.7 \\
\hline AVHRR/N-16 & 2697.45 & 1.5993E-02 & 360.54 & 361.54 & 5622.4 & $1.5711 \mathrm{E}-02$ & 359.94 & 360.94 & 5550.9 \\
\hline AVHRR/N-17 & 2670.81 & $1.5709 \mathrm{E}-02$ & 357.46 & 358.37 & 5614.9 & $1.5438 \mathrm{E}-02$ & 356.88 & 357.78 & 5544.7 \\
\hline AVHRR/N-18 & 2663.07 & $1.5627 \mathrm{E}-02$ & 356.51 & 357.44 & 5612.7 & 1.5357E-02 & 355.93 & 356.86 & 5542.3 \\
\hline GOES-08 & 2559.73 & 1.4539E-02 & 344.74 & 345.07 & 5581.6 & 1.4213E-02 & 344.01 & 344.34 & 5490.0 \\
\hline GOES-09 & 2555.64 & $1.4499 \mathrm{E}-02$ & 344.28 & 344.59 & 5581.1 & $1.4161 \mathrm{E}-02$ & 343.52 & 343.83 & 5485.7 \\
\hline GOES-10 & 2554.04 & $1.4480 \mathrm{E}-02$ & 344.07 & 344.39 & 5579.9 & $1.4132 \mathrm{E}-02$ & 343.28 & 343.60 & 5481.6 \\
\hline GOES-11 & 2561.50 & 1.4559E-02 & 344.97 & 345.29 & 5582.8 & 1.4232E-02 & 344.23 & 344.55 & 5490.9 \\
\hline GOES-12 & 2564.71 & 1.4590E-02 & 345.31 & 345.67 & 5583.1 & $1.4270 \mathrm{E}-02$ & 344.59 & 344.95 & 5493.3 \\
\hline
\end{tabular}



Table 3. Continue. Solar irradiance and effective brightness temperature for SW IR channel of AVHRR/NOAA (3B) and GOES imagers.

\begin{tabular}{|c|c|c|c|c|c|c|c|c|c|}
\hline \multirow[b]{2}{*}{ Sensor } & \multirow[b]{2}{*}{$v_{C}\left[\mathrm{~cm}^{-1}\right]$} & \multicolumn{4}{|c|}{ ASTM } & \multicolumn{4}{|c|}{ Wehrli } \\
\hline & & $\bar{S}_{0}\left[\mathrm{~W} \mathrm{~m} \mathrm{~m}^{-2} / \mathrm{cm}^{-1}\right]$ & $\mathrm{T}_{e f f}^{T O A}[\mathrm{~K}]$ & $\mathrm{T}^{*}[\mathrm{~K}]$ & $\mathrm{T}_{\text {eff }}^{A U}[\mathrm{~K}]$ & 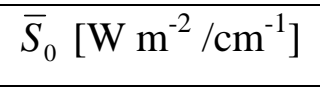 & $\mathrm{T}_{e f f}^{T O A}[\mathrm{~K}]$ & $\mathrm{T}^{*}[\mathrm{~K}]$ & $\mathrm{T}_{e f f}^{A U}[\mathrm{~K}]$ \\
\hline AVHRR/N-07 & 2685.81 & 1.5873E-02 & 359.14 & 360.16 & 5620.1 & 1.5576E-02 & 358.50 & 359.53 & 5544.2 \\
\hline AVHRR/N-08 & 2651.33 & $1.5490 \mathrm{E}-02$ & 355.10 & 356.01 & 5606.0 & 1.5243E-02 & 354.57 & 355.48 & 5541.1 \\
\hline AVHRR/N-09 & 2691.64 & $1.5937 \mathrm{E}-02$ & 359.86 & 360.86 & 5622.2 & 1.5633E-02 & 359.22 & 360.21 & 5544.8 \\
\hline AVHRR/N-10 & 2673.53 & 1.5735E-02 & 357.74 & 358.68 & 5614.8 & $1.5457 \mathrm{E}-02$ & 357.14 & 358.09 & 5543.1 \\
\hline AVHRR/N-11 & 2684.31 & 1.5855E-02 & 359.02 & 359.98 & 5619.2 & 1.5562E-02 & 358.40 & 359.35 & 5544.1 \\
\hline AVHRR/N-12 & 2653.67 & 1.5520E-02 & 355.28 & 356.31 & 5608.0 & 1.5266E-02 & 354.73 & 355.76 & 5541.3 \\
\hline AVHRR/N-14 & 2660.74 & $1.5599 \mathrm{E}-02$ & 356.08 & 357.16 & 5611.1 & $1.5334 \mathrm{E}-02$ & 355.51 & 356.59 & 5542.0 \\
\hline AVHRR/N-15 & 2694.64 & $1.5956 \mathrm{E}-02$ & 360.38 & 361.19 & 5620.2 & 1.5662E-02 & 359.76 & 360.57 & 5545.3 \\
\hline AVHRR/N-16 & 2697.45 & 1.5989E-02 & 360.53 & 361.53 & 5621.4 & 1.5689E-02 & 359.89 & 360.89 & 5545.1 \\
\hline AVHRR/N-17 & 2670.81 & 1.5705E-02 & 357.45 & 358.36 & 5613.7 & 1.5431E-02 & 356.86 & 357.77 & 5542.9 \\
\hline AVHRR/N-18 & 2663.07 & $1.5623 E-02$ & 356.50 & 357.43 & 5611.5 & $1.5356 \mathrm{E}-02$ & 355.93 & 356.86 & 5542.2 \\
\hline GOES-08 & 2559.72 & $1.4540 \mathrm{E}-02$ & 344.74 & 345.07 & 5582.2 & $1.4365 \mathrm{E}-02$ & 344.35 & 344.68 & 5532.8 \\
\hline GOES-09 & 2555.64 & $1.4502 \mathrm{E}-02$ & 344.29 & 344.59 & 5581.9 & $1.4324 \mathrm{E}-02$ & 343.89 & 344.19 & 5531.8 \\
\hline GOES-10 & 2554.04 & 1.4485E-02 & 344.08 & 344.40 & 5581.4 & $1.4308 \mathrm{E}-02$ & 343.68 & 344.00 & 5531.5 \\
\hline GOES-11 & 2561.50 & $1.4561 \mathrm{E}-02$ & 344.97 & 345.29 & 5583.3 & $1.4382 \mathrm{E}-02$ & 344.57 & 344.89 & 5533.1 \\
\hline GOES-12 & 2564.71 & $1.4590 \mathrm{E}-02$ & 345.31 & 345.67 & 5583.0 & $1.4414 \mathrm{E}-02$ & 344.92 & 345.27 & 5533.8 \\
\hline
\end{tabular}



Table 4. Coefficients of linear fit for temperature correction $\Delta T=T_{\text {eff }}-T^{*}=a+b T_{\text {eff }}$

\begin{tabular}{|c|c|c|}
\hline Sensors & $\mathrm{a}[\mathrm{K}]$ & $\mathrm{b}$ \\
\hline AVHRR/N-07 & -1.96237 & 0.00261 \\
\hline AVHRR/N-08 & -1.76976 & 0.00242 \\
\hline AVHRR/N-09 & -1.90203 & 0.00251 \\
\hline AVHRR/N-10 & -1.81648 & 0.00243 \\
\hline AVHRR/N-11 & -1.81775 & 0.00239 \\
\hline AVHRR/N-12 & -1.93614 & 0.00257 \\
\hline AVHRR/N-14 & -2.03023 & 0.00267 \\
\hline AVHRR/N-15 & -1.63603 & 0.00232 \\
\hline AVHRR/N-16* & -2.29204 & 0.00366 \\
\hline AVHRR/N-17 & -1.72194 & 0.00227 \\
\hline AVHRR/N-18 & -1.74767 & 0.00228 \\
\hline GOES-08 & -0.63544 & 8.9023E-4 \\
\hline GOES-09 & -0.58916 & 8.36325E-4 \\
\hline GOES-10 & -0.62581 & 8.87743E-4 \\
\hline GOES-11 & -0.63131 & 8.99183E-4 \\
\hline GOES-12 & -0.69539 & 9.7593E-4 \\
\hline
\end{tabular}

* Correction is nonlinear due to a spectral leak near $4.25 \mu \mathrm{m}$ for channel 3B of AVHRR/NOAA-16.

Linear fit provides accuracy within $0.1 \mathrm{~K}$. Third degree polynomial is recommended to achieve better fitting accuracy.

$$
\begin{aligned}
& \Delta T\left(T_{\text {eff }}\right)=-3.43054+0.01566 T_{\text {eff }}-3.92816 \cdot 10^{-5} T_{\text {eff }}^{2}+4.02198 \cdot 10^{-5} T_{\text {eff }}^{3} \\
& \Delta T\left(T^{*}\right)=-3.4737+0.01598 T^{*}-4.01054 \cdot 10^{-5}\left(T^{*}\right)^{2}+4.09438 \cdot 10^{-8}\left(T^{*}\right)^{3}
\end{aligned}
$$

